ERNEST DRLANDQ LAWRENEE

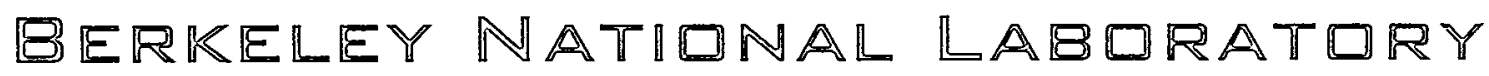

Solid-State Nuclear Magnetic

Resonance Studies of

Cross Polarization from Quadrupolar Nuclei

Susan M. De Paul

Materials Sciences Division

August 1997

Ph.D. Thesis
RECEIVED

MAR 181999

OSTI 


\section{DISCLAIMER}

This document was prepared as an account of work sponsored by the United States Government. While this document is believed to contain correct information, neither the United States Government nor any agency thereof, nor The Regents of the University of California, nor any of their employees, makes any warranty, express or implied, or assumes any legal responsibility for the accuracy, completeness, or usefulness of any information, apparatus, product, or process disclosed, or represents that its use would not infringe privately owned rights. Reference herein to any specific commercial product, process, or service by its trade name, trademark, manufacturer, or otherwise, does not necessarily constitute or imply its endorsement, recommendation, or favoring by the United States Government or any agency thereof, or The Regents of the University of California. The views and opinions of authors expressed herein do not necessarily state or reflect those of the United States Government or any agency thereof, or The Regents of the University of California.

Ernest Orlando Lawrence Berkeley National Laboratory is an equal opportunity employer. 


\section{DISCLAIMER}

\section{Portions of this document may be illegible in electronic image products. Images are produced from the best available original document.}




\title{
Solid-State Nuclear Magnetic Resonance Studies of Cross Polarization from Quadrupolar Nuclei
}

\author{
Susan Margaret De Paul \\ Ph.D. Thesis \\ Department of Chemistry \\ University of California, Berkeley \\ and \\ Materials Sciences Division \\ Ernest Orlando Lawrence Berkeley National Laboratory \\ University of California \\ Berkeley, CA 94720
}

August 1997

This work was supported by the Director, Office of Energy Research, Office of Basic Energy Sciences, Materials Sciences Division, of the U.S. Department of Energy under Contract No. DE-AC03-76SF00098. 


\title{
Solid-State Nuclear Magnetic Resonance Studies of Cross Polarization from Quadrupolar Nuclei
}

\author{
by \\ Susan Margaret De Paul \\ A.B. (Harvard University) 1992 \\ A dissertation submitted in partial satisfaction of the \\ requirements for the degree of \\ Doctor of Philosophy \\ in \\ Chemistry \\ in the \\ GRADUATE DIVISION \\ of the \\ UNIVERSITY of CALIFORNIA, BERKELEY
}

Committee in charge:

Professor Alexander Pines, Chair

Professor A. Paul Alivisatos

Professor Alexis T. Bell

Fall 1997 


\title{
Solid-State Nuclear Magnetic Resonance Studies of Cross Polarization from Quadrupolar Nuclei
}

\author{
Copyright $\odot 1997$
}

by

Susan Margaret De Paul

The U.S. Department of Energy has the right to use this document for any purpose whatsoever including the right to reproduce all or any part thereof 


\author{
Abstract \\ Solid-State Nuclear Magnetic Resonance Studies of Cross Polarization \\ from Quadrupolar Nuclei \\ by \\ Susan Margaret De Paul \\ Doctor of Philosophy in Chemistry \\ University of California, Berkeley \\ Professor Alexander Pines, Chair
}

The development of solid-state Nuclear Magnetic Resonance (NMR) has, to a large extent, focused on using spin-1/2 nuclei as probes to investigate molecular structure and dynamics. For such nuclei, the technique of cross polarization is well-established as a method for sensitivity enhancement. However, over two-thirds of the nuclei in the periodic table have a spin-quantum number greater than one-half and are known as quadrupolar nuclei. Such nuclei are fundamental constituents of many inorganic materials including minerals, zeolites, glasises, and gels. It is, therefore, of interest to explore the extent to which polarization can be transferred from quadrupolar nuclei.

In this dissertation, solid-state NMR experiments involving cross polarization from quadrupolar nuclei to spin-1/2 nuclei under magic-angle spinning (MAS) conditions are investigated in detail. The behavior of the central transition of a quadrupolar nucleus under a low-power radiofrequency spin-lock field is examined both experimentally and with numerical simulations. Complications in choosing the matching spin-lock field strength for the spin-1/2 nucleus are discussed. The dynamics of the cross-polarization process are characterized in a model compound (low albite) and a protocol for optimizing the polarization-transfer efficiency is presented. Significant enhancement of ${ }^{29} \mathrm{Si}$ NMR sensitivity by using ${ }^{27} \mathrm{Al}-$ to- $^{29} \mathrm{Si}$ and ${ }^{23} \mathrm{Na-to-}{ }^{29} \mathrm{Si}$ cross polarization is demonstrated in several inorganic compounds.

This sensitivity enhancement permits otherwise impractical two-dimensional NMR experiments to be performed. Cross polarization from quadrupolar nuclei is incorporated into experiments designed to correlate the isotropic and anisotropic parts of the chemical-shielding tensor. Several different pulse sequences for performing such 
correlations under magic-angle spinning conditions are analyzed and compared. Cross polarization from quadrupolar nuclei is also combined with the recently-developed Multiple-Quantum Magic-Angle Spinning (MQMAS) experiment to create a new technique for measuring heteronuclear correlation spectra.

In addition, the motion of cyclopentadienyl rings in four organometallic solids is studied by variable-temperature NMR, and two-dimensional exchange spectroscopy is used to demonstrate that sigmatropic rearrangements occur in the monohaptocyclopentadienyl groups of $\mathrm{Hf}\left(\eta^{5}-\mathrm{C}_{5} \mathrm{H}_{5}\right)_{2}\left(\eta^{1}-\mathrm{C}_{5} \mathrm{H}_{5}\right)_{2}$. An experiment which demonstrates that a rapid mechanical sample reorientation leads to a time reversal of radio-frequency driven spin diffusion among ${ }^{13} \mathrm{C}$ spins is also presented. 
For my parents 


\section{Table of Contents}

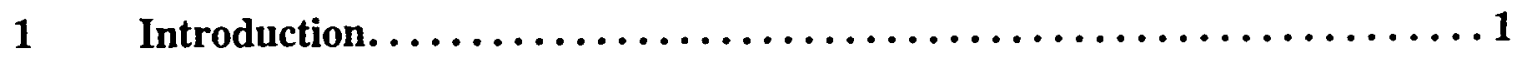

1.1 Quantum Fundamentals $\ldots \ldots \ldots \ldots \ldots \ldots \ldots \ldots \ldots \ldots \ldots \ldots \ldots \ldots \ldots \ldots \ldots \ldots$

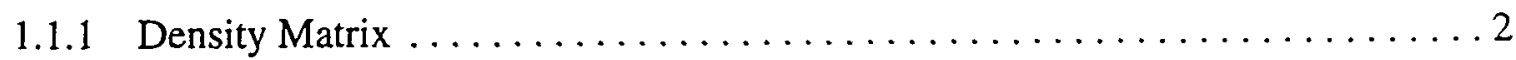

1.1 .2 Interaction Representation $\ldots \ldots \ldots \ldots \ldots \ldots \ldots \ldots \ldots \ldots \ldots \ldots \ldots \ldots \ldots \ldots \ldots$

1.1.3 Wigner Rotation Matrices............................ 10

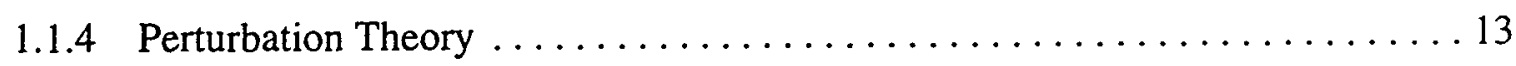

$1.2 \quad$ NMR Hamiltonians. . . . . . . . . . . . . . . . . . . . . . . . 14

1.2.1 Spin Operators and the Zeeman Interaction .................... 14

1.2.2 Rf Irradiation, Rotating Frame, and Bloch Equations $\ldots \ldots \ldots \ldots \ldots \ldots$

1.2 .3 Chemical-Shielding Interaction $\ldots \ldots \ldots \ldots \ldots \ldots \ldots \ldots \ldots \ldots \ldots \ldots \ldots \ldots \ldots$

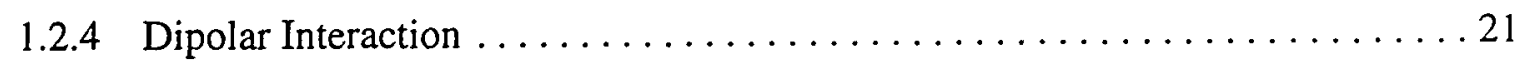

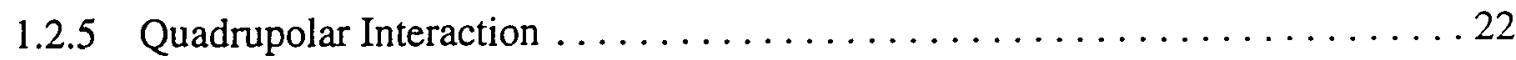

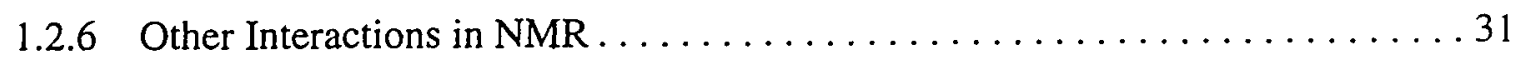

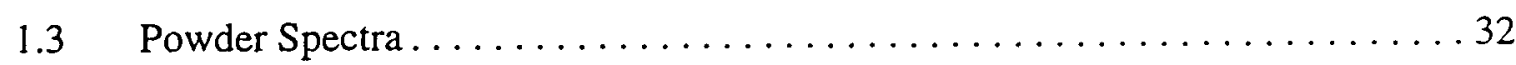

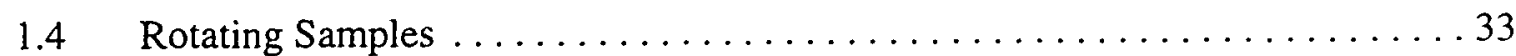

1.4.1 Wigner Rotation Matrices Revisited. ....................... 33

1.4.2 Effects of Sample Rotation on Quadrupolar Lineshapes ............... 37

$1.5 \quad$ Phase Cycling and Data Processing $\ldots \ldots \ldots \ldots \ldots \ldots \ldots \ldots \ldots \ldots \ldots \ldots \ldots \ldots \ldots$

1.5.1 Coherence-Transfer Pathways ............................... 39

1.5.2 Pure-Phase Two-Dimensional Spectra ..................... 43

$2 \quad{ }^{13} \mathrm{C}$ Variable-Temperature CP/MAS Studies of Tetracyclopentadienyl

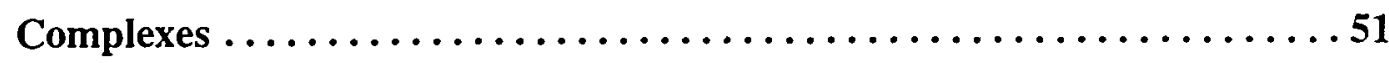

2.1 Fluxional Motion in Organometallic Compounds ............... 51

2.2 One-Dimensional ${ }^{13} \mathrm{C}$ Variable-Temperature CP/MAS Experiments . . . . . . 54

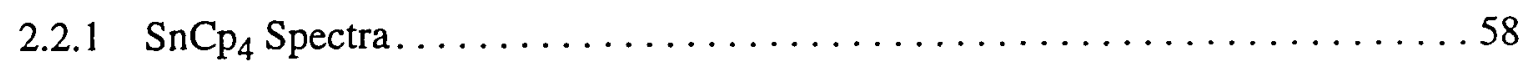

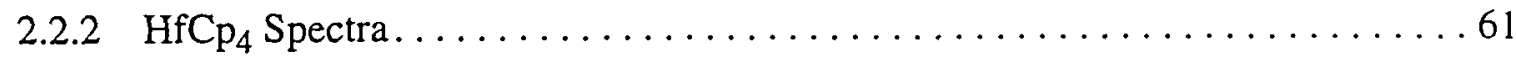

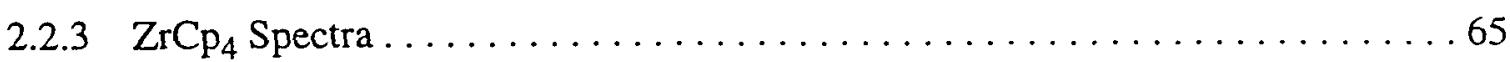

2.3 Two-dimensional Exchange Spectroscopy.................... 67 
2.4 Comparison of $\mathrm{HfCp}_{4}$ and $\mathrm{TiCp}_{4}$ Exchange Spectra $\ldots \ldots \ldots \ldots \ldots \ldots \ldots$

3 Spin Locking of Quadrupolar Nuclei During MAS. .............. 79

3.1 Low Albite as a Model Compound......................... 79

3.2 Spin Locking of Half-Integer Quadrupolar Nuclei . . . . . . . . . . . . 83

3.3 Spin Locking in the Sudden Regime $\ldots \ldots \ldots \ldots \ldots \ldots \ldots \ldots \ldots . \ldots . \ldots . \ldots$

3.4 Direct measurement of the ${ }^{27} \mathrm{Al}$ and ${ }^{23} \mathrm{Na} \mathrm{T}_{1} \rho$ 's in low albite.......... 101

4 Cross Polarization of Quadrupolar Nuclei During MAS Using Low Radiofrequency Field Amplitudes...................... 103

4.1 Previous Studies of Cross Polarization Involving Quadrupolar Nuclei . . . . . 103

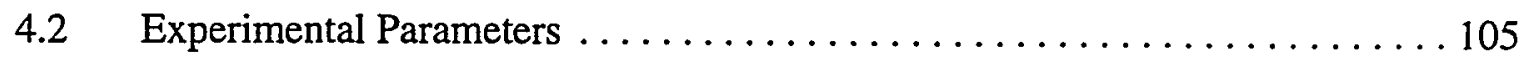

4.3 Hartmann-Hahn Matching for Quadrupolar Nuclei ............... 107

4.4 Cross-Polarization Dynamics for Quadrupolar Nuclei .............. 116

4.5 Prognosis for Cross-Polarization from Quadrupolar Nuclei Using Low-Rf Field

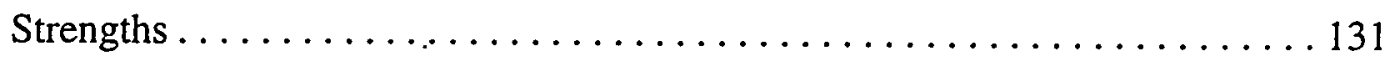

5 Applications of Cross Polarization from Quadrupolar Nuclei to

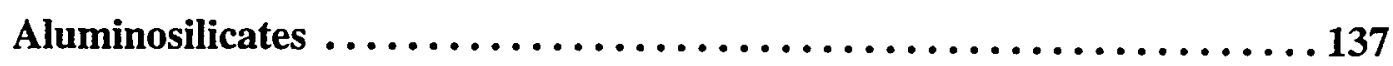

5.1 Isotropic-Anisotropic Correlation Spectroscopy ................ 137

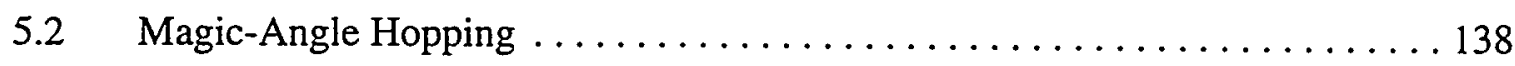

5.3 Isotropic-Anisotropic Correlation by Slow Spinning . . . . . . . . . . . 139

5.3.1 Theory of Magic-Angle Turning Experiments ................. 140

5.3.2 MAT with $90^{\circ}$ Pulses .............................. 145

5.3.3 MAT with $180^{\circ}$ Pulses ............................. 148

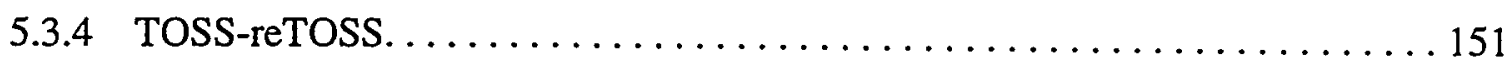

5.4 Application of Isotropic-Anisotropic Correlation Methods to Low Albite . . . 154

5.5 Experiments on Low Microcline $\ldots \ldots \ldots \ldots \ldots \ldots \ldots \ldots \ldots \ldots \ldots \ldots \ldots \ldots \ldots \ldots$

5.6 Validity of Using Cross Polarization from Quadrupolar Nuclei ......... 159

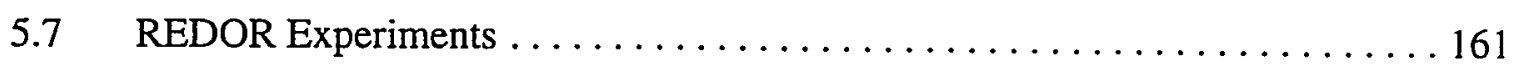


6 High-Resolution Heteronuclear Correlation between Quadrupolar and Spin1/2 Nuclei using Multiple-Quantum Magic-Angle Spinning. ........ 166

6.1 Methods for Obtaining High-Resolution Spectra of Quadrupolar Nuclei . . . . 166

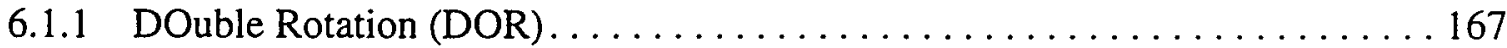

6.1.2 Dynamic-Angle Spinning (DAS) . . . . . . . . . . . . . . . . . . . 169

6.1.3 Multiple-Quantum Magic-Angle Spinning (MQMAS) . . . . . . . . . . . . 173

6.2 Heteronuclear Correlation and Quadrupolar Nuclei................ 180

6.2.1 MAS- and DAS-Based Techniques $\ldots \ldots \ldots \ldots \ldots \ldots \ldots \ldots \ldots \ldots \ldots \ldots \ldots \ldots \ldots$

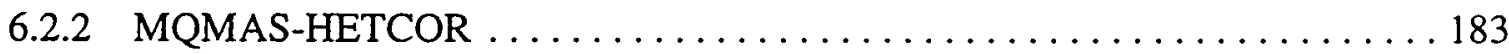

7 Reversal of Radiofrequency-Driven Spin Diffusion by Reorientation of the Sample-Spinning Axis.............................. 194

7.1 Previous Polarization-Echo Experiments . . . . . . . . . . . . . . . 194

$7.2 \quad$ Spin Diffusion.................................... 195

$7.3 \quad$ Reversal of Rf-Driven Spin Diffusion $\ldots \ldots \ldots \ldots \ldots \ldots \ldots \ldots \ldots$

7.3.1 Pulse Sequence and Experimental Apparatus................... 198

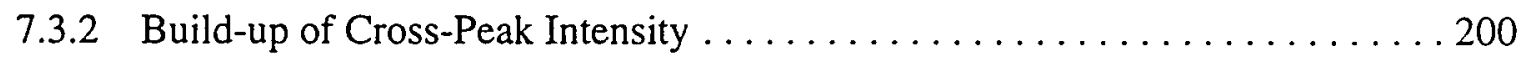

7.3.3 Spin-Diffusion Echoes . . . . . . . . . . . . . . . . . . . . . . . . . . . . 204 


\section{Acknowledgments}

This thesis would not exist without the help of many people.

First, I would like to thank my advisor, Alex, for accepting me into his group and creating an environment where it is possible to interact with and learn from a variety of talented scientists. Alex's enthusiasm is always inspiring.

I thank Dione Carmichael for keeping the group running on a day-to-day basis.

During my five years here, over sixty-five other "Pinenuts" have lived in D-62 Hildebrand, and I have probably learned something from each of them. Unfortunately, space does not permit me to acknowledge everyone individually, but I would like to mention those who directly contributed to the work presented in this thesis.

I thank Professor Jay Shore for helping me get started, for being patient with me as I blundered through my first few years of graduate school, and for intıuducing me to espresso-based drinks. He initiated the ${ }^{27} \mathrm{Al}-$ to- $^{29} \mathrm{Si}$ cross-polarization work of Chapters 3, 4, and 5. Dr. Holly Gaede and Dr. Sheryl Gann contributed to some of the early experiments, and Dr. Matthias Ernst provided direction at a crucial point in the project.

Professor Eric Munson and Dr. Marcia Ziegeweid taught me a lot about how to run spectrometers, build probes, and stay awake all night monitoring experiments while doing the work presented in Chapter 2.

The experiments in Chapter 6 were done in collaboration with Dr. Lucy Bull and soon-to-be-Dr. Shuanhu Wang. I learned from both of them, and I thank Lucy for her hospitality during the trips to Santa Barbara to finish up the project.

I thank Dr. Marco Tomaselli for teaching me about spin diffusion and echoes while doing the work presented in Chapter 7.

All of my collaborations have been pleasant experiences, and I am grateful to have had the opportunity to work with many talented scientists. I also am thankful for those group members (too numerous to mention by name) who contributed in a positive way to the atmosphere of the group. I have enjoyed the many afternoon coffee breaks at Strada (where I spent more money per year than I did on taxes!), and I have learned a lot from informal conversations. 
Friends in the department (including Eric Zylstra, Linda Brzezinski, Craig Gerken, Cindy Berrie, Ward Thompson, Pete Jacobs, Bruce Spath, Mei Hong, Eyal Barash, Carsten Vala, Amy Herhold, and Tim Germann among others) provided welcome breaks from lab over the years. I have many fond memories of sampling ethnic foods, exploring bookshops, participating in murder-mystery parties and Christmas skits, cooking Thanksgiving dinner, learning to SCUBA-dive, skiing (in the loosest sense of the word), and having interesting conversations.

I would also like to thank the group at Newman (including but not limited to Ralph and Carrie Neff, Melissa Gonzales, Gisèle Giorgi, Kevin Hartshorn, Erin Hammond, Yvette Justice, Nigel Barboza, Rex Winterbottom, Erica Boyd, Rick Harris, Felix Wu, Dave Smith, Joe Morris, Tina Ialongo, Heather Janes, and Frank Da Prato) for their friendship and support, particularly during this last year.

I thank my roommate Janet Coffman for helping to keep me sane for the past three years even in the face of bizarre situations (such as when our other roommate spontaneously decided to move to Utah in the middle of the night without telling anyone).

My parents and my sister Angie have always been there for me. Their phone calls often provide humor and much-needed perspective. I would not be where I am today without them, and I thank them for their love and support.

Last, but by no means least, I would like to thank my boyfriend Matthias for his friendship, advice, love, and encouragement during the past two years. 


\section{Chapter 1: Introduction}

This thesis primarily describes studies of the transfer of polarization from quadrupolar nuclei to spin-1/2 nuclei in high-field, solid-state Nuclear Magnetic Resonance (NMR) spectroscopy. In the first chapter, the underlying quantum-mechanical theory will be reviewed, and the manifestation of physical interactions in NMR spectra will be discussed. In Chapter 2, an example of the use of conventional solid-state NMR spectroscopy to characterize chemically interesting processes will be presented. Chapter 3 will describe experiments, simulations, and theory pertaining to the spin locking of quadrupolar nuclei in rotating samples. The information obtained from these studies can be used to optimize cross-polarization experiments as will be shown in Chapter 4 . Applications of this technique will be demonstrated in Chapters 5 and 6. Finally, a proofof-principle experiment which demonstrates that "spin diffusion" can be refocused by macroscopic sample reorientation will be presented in Chapter 7.

\subsection{Quantum Fundamentals}

One of the most exciting aspects of NMR from a spectroscopic point of view is the success of quantum mechanics in describing the behavior of nuclear spins in a magnetic field. Quantum effects can be seen more clearly in NMR than in many other branches of physical chemistry, and the effects of complicated combinations of pulses and rotations can be straightforwardly simulated. In this section, some basic ideas from quantum mechanics will be reviewed. The description provided in this thesis is neither selfcontained nor complete; in particular, a knowledge of Dirac notation is assumed. In-depth treatments of the topics presented in this section can be found in introductory books on quantum mechanics ${ }^{1,2,3}$ in monographs on $\mathrm{NMR}^{4,5,6,7,7,9}$ and in previous theses from this group. ${ }^{10,11}$ 


\subsubsection{Density Matrix}

In quantum mechanics, a particle is described by a wave function or state vector, $|\Psi(t)\rangle$. Although the wave function itself does not have a simple physical interpretation, it contains all of the information necessary to describe the particle. The wave function changes with time according to the Schrödinger equation

$$
\mathrm{i} \hbar \frac{\mathrm{d}}{\mathrm{dt}}|\Psi(\mathrm{t})\rangle=\mathscr{H}(\mathrm{t})|\Psi(\mathrm{t})\rangle
$$

where $\mathcal{H}(t)$ is the Hamiltonian operator, which describes the energy of the system.

Although, in general, the solution to the Schrödinger equation can be non-trivial, the problem is greatly simplified for the case of a time-independent Hamiltonian. In such a case, straightforward integration of Equation (1.1) gives

$$
|\Psi(\mathrm{t})\rangle=\mathrm{e}^{-\mathrm{i} \mathscr{H} / \hbar}|\Psi(0)\rangle
$$

It is possible to find a set of orthonormal state vectors, $\left\{\left|\varphi_{i}\right\rangle\right\}$, which are eigenvectors of the time-independent Hamiltonian. These state vectors obey the equation

$$
\mathcal{H}\left|\varphi_{\mathrm{i}}\right\rangle=\mathrm{E}_{\mathrm{i}}\left|\varphi_{\mathrm{i}}\right\rangle
$$

where the terms, $\left\{\mathrm{E}_{\mathrm{i}}\right\}$, are known as the eigenvalues of the Hamiltonian and correspond to the allowed energy levels for the particle.

A quantum-mechanical projection operator can be defined as follows

$$
P_{\varphi_{i}}=\left|\varphi_{i}\right\rangle\left\langle\varphi_{i}\right|
$$

When this operator is applied to a state $|\Psi(t)\rangle$, it gives the orthogonal projection of $|\Psi(t)\rangle$ onto $\left|\varphi_{\mathrm{i}}\right\rangle$. Using this projection operator, one can expand an arbitrary state, $|\Psi(\mathrm{t})\rangle$, in terms of the basis of eigenvectors of the Hamiltonian

$$
|\Psi(t)\rangle=\sum_{i}\left|\varphi_{i}\right\rangle\left\langle\varphi_{i} \mid \Psi(t)\right\rangle=\sum_{i} c_{i}(t)\left|\varphi_{i}\right\rangle
$$


This procedure is analogous to the more familiar case of describing a vector in Cartesian space by its projections onto the orthogonal $x, y$, and $z$ axes.

While the above description of a system in terms of state vectors works well for the case of a single particle, it is not well-suited to describing collections of particles. In NMR, however, one always records the signal from a large number of spins. It is, therefore, desirable to have a formalism that explicitly takes into account the statistical nature of the system. Such a formalism is provided by the density matrix..$^{2,4,12}$

The density operator is defined as

$$
\rho(t)=\sum_{k} p_{k}\left|\Psi_{k}(t)\right\rangle\left\langle\Psi_{k}(t)\right|
$$

where $p_{k}$ is the probability that the entire system is in a state $\left|\Psi_{k}(t)\right\rangle$. Note the functional similarity between Equation (1.6) and the projection operator of Equation (1.4). In this case, however, the state that is being projected onto is not necessarily an eigenstate of the Hamiltonian but is the linear superposition of eigenstates that describes the current state of the system.

If one expands $\left|\Psi_{k}(t)\right\rangle$ in the eigenbasis of the Hamiltonian (see Equation (1.5)), Equation (1.6) becomes

$$
\rho(t)=\sum_{k} \sum_{i} \sum_{j} p_{k} c_{i}^{k}(t) c_{j}^{k *}(t)\left|\varphi_{i}\right\rangle\left\langle\varphi_{j}\right|
$$

Explicitly taking the ensemble average gives

$$
\rho(t)=\sum_{i} \sum_{j} \overline{c_{i}(t) c_{j}^{*}(t)}\left|\varphi_{i}\right\rangle\left\langle\varphi_{j}\right|
$$

The statistical nature of the density operator is clearly evident in Equations (1.7) and (1.8).

The matrix elements of $\rho(t)$ in the eigenbasis of the Hamiltonian can be found using Equation (1.8). The diagonal elements 


$$
\begin{aligned}
\left\langle\varphi_{m}|\rho(t)| \varphi_{m}\right\rangle & =\sum_{i} \sum_{j} \overline{c_{i}(t) c_{j}^{*}(t)}\left\langle\varphi_{m} \mid \varphi_{i}\right\rangle\left\langle\varphi_{j} \mid \varphi_{m}\right\rangle \\
& =\sum_{i} \sum_{j} \overline{c_{i}(t) c_{j}^{*}(t)} \delta_{i m} \delta_{j m} \\
& =\mid \overline{\left.c_{m}(t)\right|^{2}}
\end{aligned}
$$

express the population of a given state $\left|\varphi_{\mathrm{m}}\right\rangle$, while the off-diagonal elements

$$
\begin{aligned}
\left\langle\varphi_{m}|\rho(t)| \varphi_{n}\right\rangle & =\sum_{i} \sum_{j} \overline{c_{i}(t) c_{j}^{*}(t)}\left\langle\varphi_{m} \mid \varphi_{i}\right\rangle\left\langle\varphi_{j} \mid \varphi_{n}\right\rangle \\
& =\sum_{i} \sum_{j} \overline{c_{i}(t) c_{j}^{*}(t)} \delta_{i m} \delta_{j n} \\
& =\overline{c_{m}(t) c_{n}^{*}(t)}
\end{aligned}
$$

express a "coherence," or interference, between two eigenstates $\left|\varphi_{m}\right\rangle$ and $\left|\varphi_{n}\right\rangle$. The concept of a coherence will be discussed in more detail in Section 1.5.1.

The time evolution of the density operator is described by the Liouville-vonNeumann equation

$$
\mathrm{i} \hbar \frac{\mathrm{d}}{\mathrm{dt}} \rho(\mathrm{t})=[\mathcal{H}(\mathrm{t}), \rho(\mathrm{t})]
$$

which can be derived from Equations (1.6) and (1.1). Equation (1.11) can be solved analytically to yield

$$
\rho(t)=U(t) \rho(0) U^{\dagger}(t)
$$

where the propagator $U(t)$ is defined as

$$
U(t)=T\left\{e^{-\frac{i}{\hbar} \int_{0}^{d} \mathscr{H}\left(t^{\prime}\right) d t^{\prime}}\right\},
$$

and the Dyson time-ordering operator, $\mathrm{T}$, specifies the order of evaluation of operators. ${ }^{13,14}$ 
The form of the initial density matrix, $\rho(0)$, in Equation (1.12) depends on the condition of the system. For a general system in thermal equilibrium, it is given by

$$
\rho(0)=\frac{e^{-\mathscr{H} / \mathrm{k}_{\mathrm{B}} \mathrm{T}}}{Z}
$$

where $\mathcal{H}$ is the Hamiltonian describing the energy levels of the system, $\mathrm{k}_{\mathrm{B}}$ is the Boltzmann constant, and $\mathrm{Z}$ is the partition function

$$
\mathrm{Z}=\operatorname{Tr}\left\{\mathrm{e}^{-\mathcal{H} / \mathrm{k}_{\mathrm{B}} \mathrm{T}}\right\}
$$

Again, the statistical nature of the density operator is readily apparent. In NMR, the energy level spacings are typically very small compared to the temperature at which the experiments are performed. Even for protons with a resonance frequency of $600 \mathrm{MHz}$, the Larmor splitting is only $h v_{L}=3.97 \times 10^{-25} \mathrm{~J}$ while the room-temperature thermal energy is $k_{B} T=4.11 \times 10^{-21} \mathrm{~J}$. This permits one to expand Equation (1.15) as

$$
\rho(0) \approx \frac{1}{Z}\left(1-\frac{\mathcal{H}}{\mathrm{k}_{\mathrm{B}} \mathrm{T}}\right)
$$

where 1 is the identity operator. Since the identity operator can neither evolve with time nor be detected in an NMR experiment, it is customarily dropped from calculations.

Expectation values can be calculated by using density matrices. Generalizing the expectation value from the Schrödinger representation to an ensemble of spins

$$
\langle A\rangle=\sum_{k} p_{k}\left\langle\psi_{k}(t)|A| \psi_{k}(t)\right\rangle
$$

and expanding the wave functions over the basis $\left|\varphi_{i}\right\rangle$ (see Equation (1.5)) gives

$$
\begin{aligned}
\langle A\rangle & =\sum_{k} \sum_{i} \sum_{j} p_{k} c_{j}^{k *}(t) c_{i}^{k}(t)\left\langle\varphi_{j}|A| \varphi_{i}\right\rangle \\
& =\sum_{i} \sum_{j} \overline{c_{j}^{*}(t) c_{i}(t)}\left\langle\varphi_{j}|A| \varphi_{i}\right\rangle .
\end{aligned}
$$


Yet the ensemble-averaged term in Equation (1.18) is simply an off-diagonal element of the density matrix (see Equation (1.10)). Equation (1.18) therefore becomes

$$
\begin{aligned}
\langle\mathrm{A}\rangle & =\sum_{\mathrm{i}} \sum_{\mathrm{j}}\left\langle\varphi_{\mathrm{i}}|\rho(\mathrm{t})| \varphi_{\mathrm{j}}\right\rangle\left\langle\varphi_{\mathrm{j}}|\mathrm{A}| \varphi_{\mathrm{i}}\right\rangle \\
& =\sum_{\mathrm{i}}\left\langle\varphi_{\mathrm{i}}|\rho(\mathrm{t}) \mathrm{A}| \varphi_{\mathrm{i}}\right\rangle \\
& =\operatorname{Tr}\{\rho(\mathrm{t}) \mathrm{A}\}
\end{aligned}
$$

Equations (1.19) and (1.11) are used fïequently in NMR to calculate the observable magnetization resulting from the application of various Hamiltonians.

\subsubsection{Interaction Representation}

The behavior of a spin system under rf pulses and sample rotation can be determined by solving the Liouville-von-Neumann equation (Equation (1.11)). Often, however, the problem can be considerably simplified by using the interaction representation to change the frame of reference. This is routinely done in NMR in the case of the rotating-frame transformation, in which the reference frame is shifted from the stationary laboratory frame to a frame that rotates with the Larmor frequency (see Section 1.2.2). In such a frame, the formerly precessing magnetization will appear stationary, and the effects of if pulses become particularly simple to describe.

The interaction representation is described in several books on NMR. ${ }^{5,8,15}$ It can be expressed in two slightly different formulations, both of which will be presented below.

Transformation to an interaction representation is possible when a Hamiltonian contains both a time-independent term and a time-dependent term

$$
\mathscr{H}=\mathcal{H}_{0}+\mathcal{H}_{1}(\mathrm{t})
$$

It is then possible to define a unitary operator, $\mathrm{R}$, as

$$
\mathrm{R}=\mathrm{e}^{-\mathrm{i} \mathcal{H}_{0} \mathrm{t} / \hbar}
$$


With this operator, one can perform a similarity transformation on the density matrix to create a new density matrix in the interaction frame

$$
\rho^{\mathrm{I}}(\mathrm{t})=\mathrm{R}^{\dagger} \rho(\mathrm{t}) \mathrm{R} .
$$

Here, use has been made of the fact that for a unitary operator

$$
R^{\dagger}=R^{-1}
$$

This ieads to the property

$$
R^{\dagger} R=R^{-1} R=\mathbf{1},
$$

which will be useful in the derivations which follow.

The interaction Hamiltonian is commonly defined as

$$
\mathscr{H}^{\mathrm{I}}(\mathrm{t})=\mathrm{R}^{\dagger} \mathcal{H}_{1}(\mathrm{t}) \mathrm{R}
$$

or as

$$
\mathscr{H}^{\mathrm{H}}(\mathrm{t})=\mathrm{R}^{\dagger} \mathcal{H}(\mathrm{t}) \mathrm{R}-\mathrm{i} \hbar \mathrm{R}^{\dagger} \frac{\mathrm{dR}}{\mathrm{dt}}
$$

Note that inclusion of the term $-\mathrm{i} \hbar \mathrm{R}^{\dagger}(\mathrm{dR} / \mathrm{dt})$ in Equation (1.26) may seem somewhat arbitrary or unphysical. However, defining the Hamiltonian in this way allows a Liouville-von-Neumann equation to be used to describe the evolution of the density matrix in the interaction frame as will be shown below. The equivalence of Equations (1.25) and (1.26) can be seen by substituting Equation (1.20) into Equation (1.26) and using both the properties of unitary operators (Equation (1.24)) and the fact that a given operator commutes with a function of that operator ${ }^{2}$ 


$$
\begin{aligned}
\mathscr{H}^{\mathrm{I}}(\mathrm{t}) & =\mathrm{R}^{\dagger}\left(\mathcal{H}_{0}+\mathscr{H}_{1}(\mathrm{t})\right) \mathrm{R}-\mathrm{i} \hbar \mathrm{R}^{\dagger} \frac{\mathrm{dR}}{\mathrm{dt}} \\
& =\mathrm{R}^{\dagger} \mathscr{H}_{0} \mathrm{R}+\mathrm{R}^{\dagger} \mathscr{H}_{1}(\mathrm{t}) \mathrm{R}-\mathrm{i} \hbar \mathrm{R}^{\dagger} \frac{\mathrm{d}}{\mathrm{dt}}\left(\mathrm{e}^{-\mathrm{i} \mathscr{H}_{0} \mathrm{t} / \hbar}\right) \\
& =\mathscr{H}_{0} \mathrm{R}^{\dagger} \mathrm{R}+\mathrm{R}^{\dagger} \mathcal{H}_{1}(\mathrm{t}) \mathrm{R}-\mathrm{i} \hbar \mathrm{R}^{\dagger}\left(\frac{-\mathrm{i} \mathcal{H}_{0}}{\hbar} \mathrm{R}\right) \\
& =\mathscr{H}_{0}+\mathrm{R}^{\dagger} \mathcal{H}_{1}(\mathrm{t}) \mathrm{R}-\mathscr{H}_{0} \mathrm{R}^{\dagger} \mathrm{R} \\
& =\mathrm{R}^{\dagger} \mathcal{H}_{1}(\mathrm{t}) \mathrm{R} .
\end{aligned}
$$

With the interaction Hamiltonian defined according to Equations (1.25) and (1.26), the density matrix in the interaction frame can be described by a Liouville-von-Neumann equation

$$
\mathrm{i} \hbar \frac{\mathrm{d}}{\mathrm{dt}} \rho^{\mathrm{I}}(\mathrm{t})=\left[\mathcal{H}^{\mathrm{I}}(\mathrm{t}), \rho^{\mathrm{I}}(\mathrm{t})\right]
$$

Equation (1.28) is the same as (1.11) as can be seen by direct substitution of Equations (1.22) and (1.25) into Equation (1.28). The left-hand side of Equation (1.28) becomes

$$
\begin{aligned}
i \hbar \frac{\mathrm{d}}{\mathrm{dt}} \rho^{\mathrm{I}}(\mathrm{t}) & =i \hbar \frac{\mathrm{d}}{\mathrm{dt}}\left(\mathrm{R}^{\dagger} \rho(\mathrm{t}) \mathrm{R}\right) \\
& =i \hbar \frac{\mathrm{d} \mathrm{R}^{\dagger}}{\mathrm{dt}} \rho(\mathrm{t}) \mathrm{R}+\mathrm{i} \hbar \mathrm{R}^{\dagger} \frac{\mathrm{d} \rho(\mathrm{t})}{\mathrm{dt}} \mathrm{R}+i \hbar \mathrm{R}^{\dagger} \rho(\mathrm{t}) \frac{\mathrm{dR}}{\mathrm{dt}} \\
& =i \hbar\left(\frac{i \mathcal{H}_{0}}{\hbar} \mathrm{R}^{\dagger}\right) \rho(\mathrm{t}) \mathrm{R}+\mathrm{i} \hbar \mathrm{R}^{\dagger} \frac{\mathrm{d} \rho(\mathrm{t})}{\mathrm{dt}} \mathrm{R}+\mathrm{i} \hbar \mathrm{R}^{\dagger} \rho(\mathrm{t})\left(\frac{-\mathrm{i} \mathcal{H}_{0}}{\hbar} \mathrm{R}\right) \\
& =i \hbar \mathrm{R}^{\dagger} \frac{\mathrm{d} \rho(\mathrm{t})}{\mathrm{dt}} \mathrm{R}-\mathrm{R}^{\dagger}\left[\mathcal{H}_{0}, \rho(\mathrm{t})\right] \mathrm{R} .
\end{aligned}
$$

Similarly, the right-hand side of Equation (1.28) becomes

$$
\begin{aligned}
{\left[\mathcal{H}^{I}(\mathrm{t}), \rho^{\mathrm{I}}(\mathrm{t})\right] } & =\left[\mathrm{R}^{\dagger} \mathcal{H}_{1}(\mathrm{t}) \mathrm{R}, \mathrm{R}^{\dagger} \rho(\mathrm{t}) \mathrm{R}\right] \\
& =\mathrm{R}^{\dagger} \mathcal{H}_{1}(\mathrm{t}) \mathrm{RR}^{\dagger} \rho(\mathrm{t}) \mathrm{R}-\mathrm{R}^{\dagger} \rho(\mathrm{t}) \mathrm{R}^{\dagger} \mathcal{H}_{1}(\mathrm{t}) \mathrm{R} \\
& =\mathrm{R}^{\dagger}\left[\mathcal{H}_{1}(\mathrm{t}), \rho(\mathrm{t})\right] \mathrm{R} .
\end{aligned}
$$


Equating the two and operating from the left by $R$ and from the right by $\mathrm{R}^{\dagger}$ gives

$$
\begin{aligned}
\mathrm{i} \hbar \frac{\mathrm{d} \rho(\mathrm{t})}{\mathrm{dt}}-\left[\mathcal{H}_{0}, \rho(\mathrm{t})\right] & =\left[\mathcal{H}_{1}(\mathrm{t}), \rho(\mathrm{t})\right] \\
\mathrm{i} \hbar \frac{\mathrm{d} \rho(\mathrm{t})}{\mathrm{dt}} & =\left[\mathcal{H}_{0}+\mathcal{H}_{1}(\mathrm{t}), \rho(\mathrm{t})\right]
\end{aligned}
$$

which is the original Liouville-von-Neumann equation (Equation (1.11)).

Note that the above derivations were rigorous and did not require any assumptions about the relative sizes of $\mathcal{H}_{0}$ and $\mathcal{H}_{1}(\mathrm{t})$. The only requirement was that $\mathcal{H}_{0}$ be timeindependent. Even this requirement need not be satisfied if Equation (1.21) is generalized to a form similar to that in Equation (1.13) although such cases will not be considered in this thesis. In many applications of this transformation, however, $\mathcal{H}_{0}$ will be the largest part of the Hamiltonian. In such cases, it is common practice to divide $\mathcal{H}_{1}(\mathrm{t})$ into a term which commutes with $\mathcal{H}_{0}, \mathscr{H}_{1}^{\mathcal{E}}(\mathrm{t})$, and a term which does not commute with $\mathcal{H}_{0}, \mathcal{H}_{1}^{\mathrm{nc}}(\mathrm{t})$, as follows

$$
\mathscr{H}_{1}(\mathrm{t})=\mathscr{H}_{1}^{\mathrm{c}}(\mathrm{t})+\mathcal{H}_{1}^{\mathrm{nc}}(\mathrm{t})
$$

The Hamiltonian in the interaction frame then becomes

$$
\mathscr{H}^{\dagger}(\mathrm{t})=\mathscr{H}_{1}^{\mathcal{E}}(\mathrm{t})+\mathrm{R}^{\dagger} \mathscr{H}_{1}^{\mathrm{nc}}(\mathrm{t}) \mathrm{R}
$$

At this point, a first-order perturbative approximation known as the secular approximation is commonly made, and it is assumed that the off-diagonal portions of the Hamiltonian are small enough relative to the diagonal elements that they can be ignored. The Hamiltonian is then truncated so that only the term $\mathcal{H}_{1}^{\varepsilon}(\mathrm{t})$ remains. ${ }^{5}$ This truncation is done in addition to the change to the interaction representation and is not a direct mathematical consequence of the transformation.

Transformation to a different frame will change the time dependence of the Hamiltonian. In the case of the rotating-frame transformation (see Section 1.2.2), the transformation eliminates the time-dependence in the if term of the lab-frame Hamiltonian. However, transformation to an interaction frame can also introduce new 
time dependences. This is precisely what happens to the terms in the original Hamiltonian that do not commute with $\mathcal{H}_{0}$ when the rotating-frame transformation is carried out. Time-independent terms which commute with $\mathcal{H}_{0}$ remain time-independent, but the noncommuting terms pick up an additional time dependence (see Equation (1.33)), which often provides further justification for making the secular approximation.

\subsubsection{Wigner Rotation Matrices}

In addition to being able to change the motional reference frame (by using the interaction representation), it is also convenient to be able to change the coordinate system to facilitate the solution of a problem. This can easily be done when spherical tensors are used. All of the physical interactions governing NMR spectra can be written in terms of sums of products of two second-rank tensors, one concerned with spin variables and one concerned with spatial variables. ${ }^{6.7}$ These tensors can then be rotated into the desired frame of reference by a unitary transformation which can be performed on each component

$$
\mathrm{U}_{\text {Euler }} \mathrm{A}_{1, \mathrm{n}} \mathrm{U}_{\text {Euler }}^{-1}
$$

where $A_{1, n}$ is the $n$-th component of a tensor of rank 1 . The unitary operator, $U_{\text {Euler }}$, represents a series of three consecutive rotations about different axes by the angles $(\alpha, \beta, \gamma){ }^{1,7,16,17,18}$ These angles are known as the Euler angles. Several different conventions are used in the literature, but the one we will use ${ }^{16}$ is depicted in Figure 1.1.

The results of applying the unitary transformation of Equation (1.34) to each component of a tensor can be represented by using Wigner rotation matrices

$$
R_{l, m}=\sum_{n=-1}^{1} D_{n, m}^{(l)}(\alpha, \beta, \gamma) A_{l, n} .
$$

Here, $R_{1, m}$ is a tensor component in the new frame; $\left\{A_{1, n}\right\}$ are the components in the old frame; and $D_{n, m}^{(1)}(\alpha, \beta, \gamma)$ is a Wigner rotation matrix. The Wigner rotation matrices can be simplified further by writing 


$$
D_{n, m}^{(1)}(\alpha, \beta, \gamma)=e^{-i(n \alpha+m \gamma)} d_{n, m}^{(1)}(\beta)
$$

where $d_{n, m}^{(1)}(\beta)$ is known as a reduced Wigner rotation matrix element and is defined according to ${ }^{16}$

$$
d_{n, m}^{(1)}(\theta)=\left\langle 1, n\left|e^{-i \theta L_{y}}\right| 1, m\right\rangle,
$$

Table 1.1 lists the reduced Wigner rotation matrix elements for $\mathrm{l}=2$. Also of interest are the matrix elements with $n=m=0$ which can be shown to simplify to ${ }^{16}$

$$
D_{0,0}^{(1)}(\alpha, \beta, \gamma)=d_{0,0}^{(1)}(\beta)=P_{1}(\cos \beta)
$$

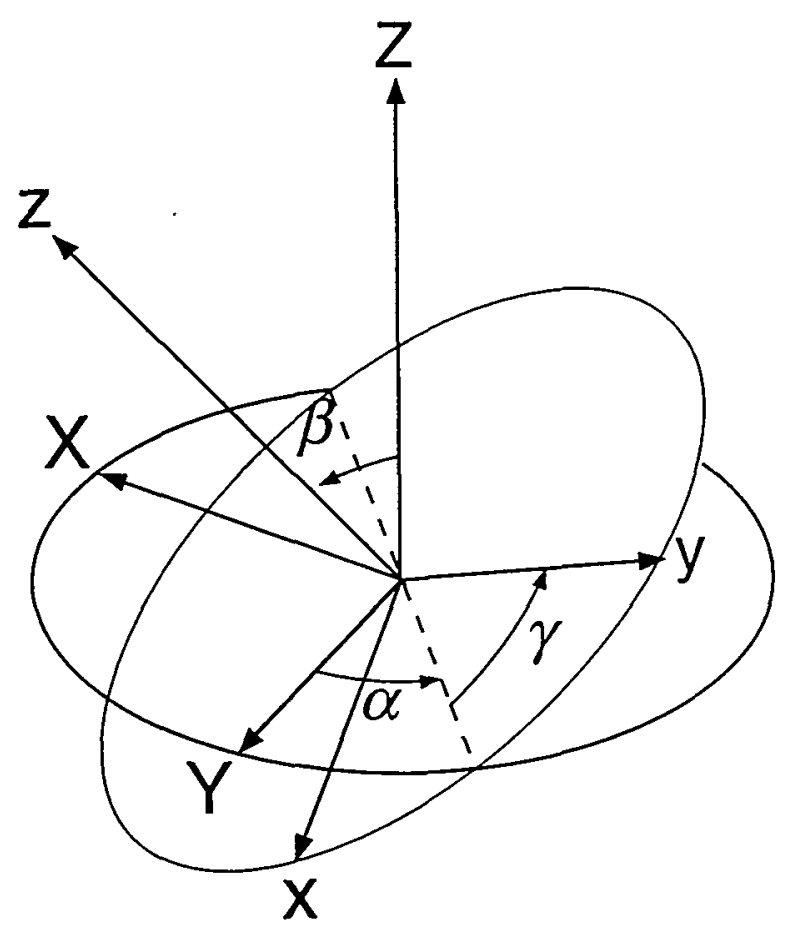

$$
(X, Y, Z) \stackrel{(\alpha, \beta, \gamma)}{\longrightarrow}(x, y, z)
$$

Figure 1.1 - Euler angle definitions. The first rotation is by an angle $\alpha$ about the $Z$ axis of the original coordinate system. This changes the positions of the $X$ and $Y$ axes. The second rotation is by the angle $\beta$ about the newly-rotated $Y$ axis, and it changes the positions of the $X$ and $Z$ axes. The third rotation is by the angle $\gamma$ about the newly-rotated $Z$ axis. The final positions of the axes are given by the labels $x, y$, and $z$. 


\begin{tabular}{|c||c|c|c|c|c|}
\hline $\mathbf{n}$ Im & 2 & $\mathbf{1}$ & $\mathbf{0}$ & -1 & -2 \\
\hline \hline 2 & $\frac{(1+\cos \beta)^{2}}{4}$ & $-\sin \beta\left(\frac{1+\cos \beta}{2}\right)$ & $\sqrt{\frac{3}{8}} \sin ^{2} \beta$ & $-\sin \beta\left(\frac{1-\cos \beta}{2}\right)$ & $\frac{(1-\cos \beta)^{2}}{4}$ \\
\hline $\mathbf{1}$ & $\sin \beta\left(\frac{1+\cos \beta}{2}\right)$ & $\cos ^{2} \beta-\left(\frac{1-\cos \beta}{2}\right)$ & $-\sqrt{\frac{3}{8}} \sin 2 \beta$ & $\left(\frac{1+\cos \beta}{2}\right)-\cos ^{2} \beta$ & $-\sin \beta\left(\frac{1-\cos \beta}{2}\right)$ \\
\hline $\mathbf{0}$ & $\sqrt{\frac{3}{8}} \sin ^{2} \beta$ & $\sqrt{\frac{3}{8}} \sin 2 \beta$ & $\frac{3 \cos ^{2} \beta-1}{2}$ & $-\sqrt{\frac{3}{8}} \sin 2 \beta$ & $\sqrt{\frac{3}{8}} \sin ^{2} \beta$ \\
\hline-1 & $\sin \beta\left(\frac{1-\cos \beta}{2}\right)$ & $\left(\frac{1+\cos \beta}{2}\right)-\cos ^{2} \beta$ & $\sqrt{\frac{3}{8}} \sin ^{2} \beta$ & $\cos ^{2} \beta-\left(\frac{1-\cos \beta}{2}\right)$ & $-\sin \beta\left(\frac{1+\cos \beta}{2}\right)$ \\
\hline-2 & $\frac{(1-\cos \beta)^{2}}{4}$ & $\sin \beta\left(\frac{1-\cos \beta}{2}\right)$ & $\sqrt{\frac{3}{8}} \sin ^{2} \beta$ & $\sin \beta\left(\frac{1+\cos \beta}{2}\right)$ & $\frac{(1+\cos \beta)^{2}}{4}$ \\
\hline
\end{tabular}

Table 1.1 - Reduced Wigner rotation matrix elements $d_{n, m}^{(2)}(\beta)$.

where the $\mathrm{P}_{1}(\cos \beta)$ terms are known as Legendre polynomials. The relevant Legendre polynomials for NMR are the zeroth-, second-, and fourth- rank terms which are given by

$$
\begin{gathered}
\mathrm{P}_{0}(\cos \beta)=1 \\
\mathrm{P}_{2}(\cos \beta)=\frac{1}{2}\left(3 \cos ^{2} \beta-1\right)
\end{gathered}
$$

and

$$
P_{4}(\cos \beta)=\frac{1}{8}\left(35 \cos ^{4} \beta-30 \cos ^{2} \beta+3\right)
$$

The Principal-Axis System (PAS) for a given interaction is the frame of reference in which the space tensor is diagonal. It is, therefore, a convenient representation to use when describing individual crystallites, but in general it does not correspond to what is observed in the laboratory. The Wigner rotation matrices are used in NMR to relate the orientation of a given crystallite to the lab frame or to the axis about which the macroscopic sample is rotated (see Section 1.4). Many examples of their use will be seen throughout this thesis. 


\subsubsection{Perturbation Theory}

The derivation of non-degenerate, time-independent perturbation theory can be found in any textbook on quantum mechanics ${ }^{1,2}$ and only the key results will be summarized below.

Perturbation theory can be applied to solve the time-independent Schrödinger equation (Equation (1.3)) when the Hamiltonian has the form

$$
\mathcal{H}=\mathcal{H}_{0}+\mathrm{V}
$$

where the magnitude of $\mathrm{V}$ is very small compared to that of $\mathcal{H}_{0}$ and where the Schrödinger equation for $\mathcal{H}_{0}$ can be solved exactly

$$
\mathcal{H}_{0}|j\rangle=E_{j}^{(0)}|j\rangle .
$$

The eigenvalues of the full Hamiltonian (Equation (1.42)) can then be shown to be approximated by

$$
E_{j}=E_{j}^{(0)}+E_{j}^{(1)}+E_{j}^{(2)}+\ldots
$$

where the first-order correction to the energy is given by

$$
E_{j}^{(1)}=\langle j|V| j\rangle
$$

and the second-order correction to the energy is given by

$$
E_{j}^{(2)}=\sum_{k \neq j} \frac{\langle j|V| k\rangle\langle k|V| j\rangle}{E_{j}^{(0)}-E_{k}^{(0)}} .
$$

For many cases in NMR, only first-order corrections need to be taken into account to describe the observed spectrum. However, for the case of the quadrupolar interaction (see Section 1.2.5), second-order terms need to be explicitly considered. 
For time-dependent interactions, other forms of perturbation theory need to be considered. These include Fermi's Golden Rule, ${ }^{2}$ Average-Hamiltonian Theory (AHT), ${ }^{4,6}$ and Floquet theory. ${ }^{19,20,21,22}$ The latter two are suitable only for periodic Hamiltonians, a condition which often occurs in NMR. Note that the zeroth-order average Hamiltonian is given by

$$
\tilde{\mathcal{H}}^{(0)}=\frac{1}{\tau} \int_{0}^{\tau} \mathscr{H}(\mathrm{t}) \mathrm{dt}
$$

where $\tau$ is period of the cycle. This is equivalent to the result from first-order static perturbation theory, and the two descriptions will be used interchangeably throughout this thesis.

\subsection{NMR Hamiltonians}

While the concepts presented in Section 1.1 are powerful tools for calculating the quantum-mechanical behavior of a system, they require a knowledge of the Hamiltonians that govern the physical behavior of the system. In this section, a brief description is given of some important interactions in solid-state NMR and the corresponding Hamiltonians. The Hamiltonians are expressed in terms of sums of products of spherical tensors in a manner similar to that described by Haeberlen. ${ }^{67,11}$

\subsubsection{Spin Operators and the Zeeman Interaction}

By the laws of angular momentum, a nucleus with a spin-quantum number I has $(2 I+1)$ eigenstates. These states are labeled by the quantum number $m$ where $m$ has one of the values $\{-\mathrm{I},-\mathrm{I}+1, \ldots, \mathrm{I}-1, \mathrm{I}\}$. In the presence of an externally applied static magnetic field, these states become non-degenerate due to the interaction of the spin with the magnetic field. This interaction is known as the Zeeman interaction and can be described by the following Hamiltonian

$$
\mathcal{H}_{\mathrm{Z}}=-\hbar \gamma \mathrm{B}_{0} \mathrm{I}_{\mathrm{z}}
$$


where $\gamma$ is the isotope-dependent gyromagnetic ratio and $\mathrm{B}_{0}$ is the strength of the applied magnetic field. Here we have used the usual convention that the direction of the magnetic field defines the $\mathrm{z}$-axis. The operator, $\mathrm{I}_{\mathrm{z}}$, is the operator for the $\mathrm{z}$-component of the angular momentum and has eigenvalues and eigenvectors given by

$$
\mathrm{I}_{\mathrm{z}}|\mathrm{I}, \mathrm{m}\rangle=\mathrm{m}|\mathrm{I}, \mathrm{m}\rangle
$$

Since the Zeeman Hamiltonian is by far the largest Hamiltonian in all cases discussed in this thesis, its eigenvectors can be used as a basis set, and all other interactions can be treated as perturbations to the Zeeman Hamiltonian (see Section 1.1.4). By first-order, time-dependent perturbation theory, ${ }^{4}$ it is then possible to show that the selection rule for magnetic resonance transitions is $\Delta \mathrm{m}= \pm 1$ (although "forbidden" transitions can be excited to some extent as predicted by higher-order terms in the perturbation expansion). Thus, the NMR transition frequency is $\omega_{L}=-\gamma B_{0}$ and is known as the Larmor frequency. It corresponds to the frequency of precession of the spins about the $\mathrm{B}_{0}$ field.

The transverse angular-momentum operators, $I_{x}$ and $I_{y}$, are important in NMR since all observable magnetization is proportional to them. They are not diagonal in this basis, however, and their non-zero matrix elements are given by

$$
\begin{aligned}
\left\langle I, m\left|I_{x}\right| I, m^{\prime}\right\rangle= & \frac{1}{2}\left\{\sqrt{I(I+1)-m^{\prime}\left(m^{\prime}+1\right)} \delta_{m, m^{\prime}+1}\right. \\
& \left.+\sqrt{I(I+1)-m^{\prime}\left(m^{\prime}-1\right)} \delta_{m, m^{\prime}-1}\right\}
\end{aligned}
$$

and

$$
\begin{aligned}
\left\langle I, m\left|I_{y}\right| I, m^{\prime}\right\rangle= & \frac{1}{2 \mathrm{i}}\left\{\sqrt{\mathrm{I}(\mathrm{I}+1)-\mathrm{m}^{\prime}\left(\mathrm{m}^{\prime}+1\right)} \delta_{\mathrm{m}, \mathrm{m}^{\prime}+1}\right. \\
& \left.-\sqrt{\mathrm{I}(\mathrm{I}+1)-\mathrm{m}^{\prime}\left(\mathrm{m}^{\prime}-1\right)} \delta_{\mathrm{m}, \mathrm{m}^{\prime}-1}\right\} .
\end{aligned}
$$

Often it is convenient to use linear combinations of $\mathrm{I}_{\mathrm{x}}$ and $\mathrm{I}_{\mathrm{y}}$ as follows

$$
\mathrm{I}_{+}=\mathrm{I}_{\mathrm{x}}+\mathrm{iI}_{\mathrm{y}}
$$




$$
I_{-}=I_{x}-i I_{y} \text {. }
$$

The operators defined in Equations (1.52) and (1.53) are known as raising and lowering operators, respectively, since they change the magnetic quantum number by one

$$
\begin{aligned}
& I_{+}|I, m\rangle=\sqrt{I(I+1)-m(m+1)}|I, m+1\rangle \\
& I_{-}|I, m\rangle=\sqrt{I(I+1)-m(m-1)}|I, m-1\rangle .
\end{aligned}
$$

The spherical tensor operators that will be used in Sections 1.2.3, 1.2.4, and 1.2.5 can easily be expressed in terms of the operators $\mathrm{I}_{+}, \mathrm{I}_{-}$, and $\mathrm{I}_{z}$

\subsubsection{Rf Irradiation, Rotating Frame, and Bloch Equations}

Transitions between the eigenstates of the Zeeman Hamiltonian can be induced by application of a linearly polarized if field perpendicular to the static $\mathrm{B}_{0}$ field. Such a field can be described by the following Hamiltonian

$$
\mathcal{H}_{\mathrm{RF}}=-\hbar 2 \gamma_{\mathrm{I}} \mathrm{B}_{1 \mathrm{I}} \cos \left(\omega_{\mathrm{C}} \mathrm{t}+\phi\right) \mathrm{I}_{\mathrm{x}}
$$

where $2 \mathrm{~B}_{1 \mathrm{II}}$ is the strength of the applied If field, and $\phi$ is an arbitrary phase. The frequency $\omega_{C}$ is the carrier frequency of the rf; to induce a transition, $\omega_{C}$ must be nearly resonant with the Larmor frequency of the I spins $\left(\omega_{\mathrm{L}}\right)$. The if irradiation can be decomposed into the sum of two circularly polarized components rotating at frequencies $+\omega_{C}$ and $-\omega_{C}$, leading to

$$
\mathcal{H}=\mathscr{H}_{Z}+\mathscr{H}_{R F}=\hbar \omega_{L} I_{z}+\hbar \omega_{1 I}\left[e^{i \omega_{c} t} e^{i \phi}+e^{-i \omega_{c} t} e^{-i \phi}\right] I_{x}
$$

which describes the combined effects of the Zeeman and if fields; note that $\omega_{1 I}=-\gamma_{I} B_{1 I}$. Only the component of the rf field that rotates in the same direction as the precessing moment will induce transitions between the Zeeman levels. To see explicitly how the if irradiation affects the spins, it is convenient to switch to an interaction 
representation in which this component is stationary. This can be accomplished by using the transformation $\mathrm{R}=\mathrm{e}^{-\mathrm{i} \omega_{\mathrm{C}} \mathrm{tI} z}$ along with Equation (1.26). Equation (1.57) then becomes

$$
\begin{aligned}
\tilde{\mathcal{H}}= & \hbar\left(\omega_{\mathrm{L}}-\omega_{\mathrm{C}}\right) \mathrm{I}_{\mathrm{z}}+\hbar \omega_{1 \mathrm{I}}\left[\left(\mathrm{I}_{\mathrm{x}} \cos \phi-\mathrm{I}_{\mathrm{y}} \sin \phi\right)\right. \\
& +\left(\mathrm{I}_{\mathrm{x}} \cos \left(2 \omega_{\mathrm{C}} \mathrm{t}+\phi\right)-\mathrm{I}_{\mathrm{y}} \sin \left(2 \omega_{\mathrm{C}} \mathrm{t}+\phi\right)\right)
\end{aligned}
$$

Note that the coordinate system is right-handed with respect to rotations about $\omega_{1 I}$ (which points in the opposite direction from the magnetic field for spins with a positive gyromagnetic ratio). ${ }^{4}$ Neglecting the terms which oscillate with $2 \omega_{C}$ leaves a stationary field that the magnetization can precess about. When $\omega_{L}=\omega_{C}$ (on-resonance irradiation), this field lies in the xy-plane.

This frame of reference is known as the "rotating frame," and it is commonly used to describe NMR experiments. It is more than a mathematical convenience: the mixing process in the receiver of an NMR spectrometer subtracts out the carrier frequency so that the recorded signal corresponds to the magnetization in the $x y$-plane of the rotating frame. All Hamiltonians in this thesis will be written in this rotating frame. Note that there is another common usage of the words "rotating frame," however, which arises in the context of spin-locked magnetization. Experiments in which the relevant axis of quantization is defined by the rf field rather than the Zeeman field are often said to take place in the "rotating frame." With the exception of the term "rotating-frame relaxation" $\left(T_{1 p}\right)$, only the former definition will be used in this thesis.

So far, the treatment of NMR in this thesis has been entirely quantum-mechanical. However, a classical picture of magnetization vectors precessing about magnetic fields is also useful. The classical viewpoint is best summarized by the Bloch equations, which are written in the rotating frame as follows 


$$
\begin{gathered}
\frac{d M_{x}}{d t}=\gamma\left(M_{y} B_{z}-M_{z} B_{y}\right)-\frac{M_{x}}{T_{2}} \\
\frac{d M_{y}}{d t}=\gamma\left(M_{z} B_{x}-M_{x} B_{z}\right)-\frac{M_{y}}{T_{2}} \\
\frac{d M_{z}}{d t}=\gamma\left(M_{x} B_{y}-M_{y} B_{x}\right)-\frac{\left(M_{z}-M_{0}\right)}{T_{1}}
\end{gathered}
$$

where $M_{0}$ is the equilibrium magnetization; $\left(B_{x}, B_{y}, B_{z}\right)$ are the components of the magnetic field in the rotating frame; $T_{1}$ is the longitudinal relaxation time; and $T_{2}$ is the transverse relaxation time. The theory of relaxation ${ }^{8}$ has been extensively developed but is beyond the scope of this thesis. From the point of view of the experiments discussed below, the values of $T_{1}$ and $T_{2}$ will appear most prominently as practical constraints which influence the choice of pulse sequence for a given application.

\subsubsection{Chemical-Shielding Interaction}

The chemical shift reflects the magnetic shielding of a nucleus by neighboring electrons. Like the Zeeman interaction, the chemical shift is proportional to the strength of the applied magnetic field. Both diamagnetic and paramagnetic effects contribute to the chemical shift, which is an orientation-dependent quantity. In solution-state NMR spectroscopy, rapid molecular tumbling averages out this orientation-dependence and produces a narrow line at a position, known as the isotropic chemical shift, which reflects. the average electronic environment of the nucleus. In the spectra of powdered solid samples, however, individual crystallites have different orientations with respect to $B_{0}$ and, consequently, slightly different resonance frequencies.

The Hamiltonian for the full chemical-shielding interaction in the laboratory frame is given by

$$
\mathcal{H}_{\mathrm{CS}}=\hbar \gamma \sum_{1=0,2} \sum_{\mathrm{m}=-2}^{2}(-1)^{\mathrm{m}} \mathrm{R}_{\mathrm{l},-\mathrm{m}}^{\mathrm{CS}} \mathrm{T}_{\mathrm{l}, \mathrm{m}}^{\mathrm{CS}}
$$


Because this Hamiltonian is symmetric, no $l=1$ terms are present in the sum. ${ }^{1,16}$ This is generally true for NMR Hamiltonians. ${ }^{6}$ The spatial terms in Equation (1.60) can be calculated using Equation (1.35)

$$
R_{l,-m}^{C S}=\sum_{n=-1}^{l} D_{n,-m}^{(1)}\left(\alpha^{C S}, \beta^{C S}, \gamma^{C S}\right) \rho_{1, n}^{c s}
$$

where the spherical-tensor components in the principal-axis system are given by

$$
\begin{gathered}
\rho_{0,0}^{C S}=\frac{1}{3}\left(\sigma_{x x}+\sigma_{y y}+\sigma_{z z}\right)=\sigma_{i s o} \\
\rho_{2,0}^{C S}=\sqrt{\frac{3}{2}}\left[\sigma_{z z}-\frac{1}{3}\left(\sigma_{x x}+\sigma_{y y}+\sigma_{z z}\right)\right]=\sqrt{\frac{3}{2}} \delta^{C S} \\
\rho_{2, \pm 1}^{C S}=0 \\
\rho_{2, \pm 2}^{C S}=\frac{1}{2}\left[\frac{\left(\sigma_{y y}-\sigma_{x x}\right)}{\left(\sigma_{z z}-\sigma_{i s o}\right)}\right] \delta^{C S}=\frac{1}{2} \eta^{C S} \delta^{C S} .
\end{gathered}
$$

The term $\eta^{C S}$ is known as the asymmetry parameter and represents the deviation of the chemical-shielding interaction from cylindrical symmetry. The term $\delta^{C S}$ is known as the anisotropy parameter. The spin part can be expressed $a s^{6}$

$$
\begin{gathered}
\mathrm{T}_{0,0}^{C S}=\mathrm{I}_{\mathrm{z}} \mathrm{B}_{0} \\
\mathrm{~T}_{2,0}^{\mathrm{CS}}=\sqrt{\frac{2}{3}} \mathrm{I}_{\mathrm{z}} \mathrm{B}_{0} \\
\mathrm{~T}_{2, \pm 1}^{\mathrm{CS}}=\mp \frac{1}{2} \mathrm{I}_{ \pm} \mathrm{B}_{0} \\
\mathrm{~T}_{2, \pm 2}^{\mathrm{CS}}=0 .
\end{gathered}
$$

Several conventions are used in the literature for describing the principal values of the chemical-shielding anisotropy (CSA). The one used in Equation (1.62) is

$$
\left|\sigma_{z z}-\sigma_{i s o}\right| \geq\left|\sigma_{x x}-\sigma_{i s o}\right| \geq\left|\sigma_{y y}-\sigma_{i s o}\right| .
$$


This has the effect that $\eta^{C S}$ will always be a positive number between zero and one. However, whether the most downfield shift is $\sigma_{\mathrm{zz}}$ or $\sigma_{\mathrm{xx}}$ depends on which side of $\sigma_{\mathrm{iso}}$ the term $\sigma_{\mathrm{yy}}$ is on. ${ }^{23}$ An alternate convention has therefore been defined as follows

$$
\left|\sigma_{11}\right| \geq\left|\sigma_{22}\right| \geq\left|\sigma_{33}\right|
$$

Using this convention, $\sigma_{11}$ is always the most downfield term. The definition of $\eta^{C S}$ now depends on the relative magnitudes of $\left|\sigma_{11}-\sigma_{\text {iso }}\right|$ and $\left|\sigma_{33}-\sigma_{i s o}\right|{ }^{24}$ If $\left|\sigma_{11}-\sigma_{\text {iso }}\right|>$ $\left|\sigma_{33}-\sigma_{i s o}\right|$, the following identifications can be made: $\sigma_{11}=\sigma_{z z}, \sigma_{22}=\sigma_{y y}$, and $\sigma_{33}=\sigma_{x x}$. If $\left|\sigma_{33}-\sigma_{\text {iso }}\right|>\left|\sigma_{11}-\sigma_{i s o}\right|$, the proper correspondence is: $\sigma_{33}=\sigma_{z z}, \sigma_{22}=\sigma_{y y}$, and $\sigma_{11}=\sigma_{x x}$.

We will use the convention of Equation (1.64) when describing any theoretical aspects of chemical shifts, but in Chapter 5 measurements will be reported using the convention of Equation (1.65), as is customary.

Because the chemical-shielding anisotropy is on the order of several $\mathrm{kHz}$, it can be considered as a perturbation to the Zeeman Hamiltonian. It is, therefore, useful to transform into a frame rotating with the Larmor frequency (see Section 1.2.2). Ignoring all oscillating terms (i.e. - making the secular approximation) leaves only terms proportional to the spin tensors $\mathrm{T}_{0,0}^{\mathrm{CS}}$ and $\mathrm{T}_{2,0}^{\mathrm{CS}}$

$$
\begin{aligned}
\mathcal{H}_{C S}= & \hbar \gamma\left\{\mathrm{T}_{0,0}^{\mathrm{CS}} \mathrm{D}_{0,0}^{(0)}\left(\alpha^{\mathrm{CS}}, \beta^{\mathrm{CS}}, \gamma^{\mathrm{CS}}\right) \rho_{2,0}^{\mathrm{CS}}\right. \\
& \left.+\mathrm{T}_{2,0}^{\mathrm{CS}} \sum_{\mathrm{n}=-2}^{2} \mathrm{D}_{\mathrm{n}, 0}^{(2)}\left(\alpha^{\mathrm{CS}}, \beta^{\mathrm{CS}}, \gamma^{\mathrm{CS}}\right) \rho_{2, \mathrm{n}}^{\mathrm{CS}}\right\}
\end{aligned}
$$

which simplifies to

$$
\mathscr{H}_{\mathrm{CS}}=\hbar \gamma \mathrm{B}_{0} \mathrm{I}_{\mathrm{z}}\left\{\sigma_{\text {iso }}+\delta^{\mathrm{CS}}\left[\frac{\left(3 \cos ^{2} \beta^{\mathrm{CS}}-1\right)}{2}+\frac{\eta}{2} \sin ^{2} \beta^{\mathrm{CS}} \cos 2 \alpha^{\mathrm{CS}}\right]\right\} .
$$

Note that the chemical-shielding Hamiltonian is orientation-dependent. 


\subsubsection{Dipolar Interaction}

Another important interaction in solid-state NMR is the through-space interaction between the magnetic moments of two nuclei. This interaction can be described by the following Hamiltonian

$$
\mathcal{H}_{\mathrm{D}}=-\left(\frac{\mu_{0}}{4 \pi}\right) 2 \hbar^{2} \gamma_{\mathrm{j}} \gamma_{\mathrm{k}} \sum_{\mathrm{l}=0,2} \sum_{\mathrm{m}=-2}^{2}(-1)^{\mathrm{m}} \mathrm{R}_{\mathrm{l},-\mathrm{m}}^{\mathrm{D}} \mathrm{T}_{\mathrm{l}, \mathrm{m}}^{\mathrm{D}}
$$

where the $R_{l,-m}^{D}$ are described in an analogous way to the chemical-shift case (see Equation (1.61)) and the spatial components in their PAS are given by

$$
\begin{gathered}
\rho_{0,0}^{D}=\rho_{2, \pm 1}^{D}=\rho_{2, \pm 2}^{D}=0 \\
\rho_{2,0}^{D}=\sqrt{\frac{3}{2}} \delta^{D}=\sqrt{\frac{3}{2}} \frac{1}{3}
\end{gathered}
$$

while the spin components have the form

$$
\begin{gathered}
T_{0,0}^{D}=\vec{I}_{j} \cdot \vec{I}_{k} \\
T_{2,0}^{D}=\frac{1}{\sqrt{6}}\left[3 I_{j z} I_{k z}-\vec{I}_{j} \cdot \vec{I}_{k}\right] \\
T_{2, \pm 1}^{D}=\mp \frac{1}{2}\left[I_{j \pm} I_{k z}+I_{j z} I_{k \pm}\right] \\
T_{2, \pm 2}^{D}=\frac{1}{2} I_{j \pm} I_{k \pm} .
\end{gathered}
$$

Since $\rho_{0,0}^{D}$ equals zero, there is no isotropic dipolar coupling. Also, the interaction is cylindrically symmetric about the internuclear vector $\left(\eta^{D}=0\right)$. Like the chemical shift, the strength of the dipolar interaction is small (tens of $\mathrm{kHz}$ ) relative to the Zeeman interaction, allowing first-order perturbation theory to be applied. Assuming that the two spins are of the same type (homonuclear), one can make the secular approximation which will retain only the terms proportional to $T_{2,0}^{\mathrm{D}}$ 


$$
\mathscr{H}_{\mathrm{D}}=-\hbar\left\{\left(\frac{\mu_{0}}{4 \pi}\right) \frac{\hbar \gamma_{\mathrm{j}} \gamma_{\mathrm{k}}}{\mathrm{r}_{\mathrm{jk}}^{3}}\right\} \frac{\left(3 \cos ^{2} \beta^{\mathrm{D}}-1\right)}{2}\left[3 \mathrm{I}_{\mathrm{jz}} \mathrm{I}_{\mathrm{kz}}-\overrightarrow{\mathrm{I}}_{\mathrm{j}} \cdot \overrightarrow{\mathrm{I}}_{\mathrm{k}}\right]
$$

where the term in curly brackets is the dipolar-coupling constant, $\mathrm{d}_{\mathrm{jk}}$ (in units of frequency). If the two spins are of different types, then not even $\mathrm{T}_{2,0}^{\mathrm{D}}$ will commute with the Zeeman term. However, it is possible to rewrite $\mathrm{T}_{2,0}^{D}$ as a sum of a commuting and a non-commuting term (see Equation (1.32))

$$
\mathrm{T}_{2,0}^{\mathrm{D}}=\frac{1}{\sqrt{6}}\left[2 \mathrm{I}_{\mathrm{jz}} \mathrm{I}_{\mathrm{kz}}-\frac{1}{2}\left(\mathrm{I}_{\mathrm{j}+} \mathrm{I}_{\mathrm{k}-}+\mathrm{I}_{\mathrm{j}-\mathrm{k}+} \mathrm{I}_{\mathrm{k}}\right)\right]
$$

The first term on the right commutes with the Zeeman Hamiltonian even for unlike spins. The second term is often called the "flip-flop" term since it corresponds to an exchange of magnetization between two spins. It is not energy-conserving for unlike spins (unless special pulse sequences are applied) and, therefore, must be dropped from the Hamiltonian when the secular approximation is made in heteronuclear spin systems.

\subsubsection{Quadrupolar Interaction}

In addition to the magnetic interactions described in the previous sections, electrostatic interactions can also influence the fine structure of an NMR spectrum. The interaction of a nucleus with a surrounding local electric-field gradient is known as the quadrupolar interaction. The strength of this interaction can be as large as hundreds of $\mathrm{MHz}$, making it comparable to or greater than the Zeeman interaction. In such cases, the quadrupolar Hamiltonian can provide the axis of quantization, and rf-irradiation is used to excite transitions between the various levels. This type of spectroscopy is known as Nuclear Quadrupole Resonance (NQR) spectroscopy. It will not be discussed further in this thesis, but an excellent introduction to the topic can be found in the monograph of Das and Hahn. 25

We will be concerned with the case where the quadrupolar interaction is small enough to be treated as a perturbation to the Zeeman splitting although it will typically be large enough that second-order terms must be explicitly considered. Because experiments involving quadrupolar nuclei comprise the majority of this thesis, the physical basis for 
the quadrupolar interaction will be outlined following the derivation of Cohen and Reif, $5,10,26$ and then the quantum-mechanical Hamiltonian will be presented. Finally, some of the issues relating to excitation and observation of the central transition of an odd-halfinteger quadrupolar nucleus will be discussed. ${ }^{27}$

The classical energy of interaction of a chargc density, $\rho(\mathbf{r})$, with an electrostatic potential, $\mathrm{V}(\mathbf{r})$, can be expressed as

$$
E=\int d^{3} \mathbf{r} \rho(\mathbf{r}) V(\mathbf{r})
$$

If the potential is expanded about the center of mass of the nucleus, Equation (1.73) becomes

$$
\begin{aligned}
E=V(0) \int d^{3} \mathbf{r} \rho(\mathbf{r}) & +\left.\sum_{\alpha=1}^{3}\left(\frac{d V}{d x_{\alpha}}\right)\right|_{r=0} \int_{r=0}^{3} \mathbf{r} \rho(\mathbf{r}) x_{\alpha} \\
& +\left.\frac{1}{2 !} \sum_{\alpha=1}^{3} \sum_{\beta=1}^{3}\left(\frac{d^{2} V}{d x_{\alpha} d x_{\beta}}\right)\right|_{r=0} \int d^{3} \mathbf{r} \rho(\mathbf{r}) x_{\alpha} x_{\beta}+\ldots
\end{aligned}
$$

where the $\mathrm{x}_{\alpha}$ are Cartesian components. The first term on the right-hand side Equation (1.74) is the interaction of a point-charge nucleus (an electric monopole) with a constant potential; it will not affect the NMR spectrum. ${ }^{26}$ The second term, an electric-dipole term, vanishes because it has odd parity. ${ }^{1}$ But the third term, the electric quadrupolar interaction, can be non-zero. It is the product of two tensors: a quadrupole moment with components

$$
Q_{\alpha \beta}{ }^{\prime}=\int d^{3} \mathbf{r} \rho(\mathbf{r}) x_{\alpha} x_{\beta}
$$

and an electric field gradient with components

$$
V_{\alpha \beta}=\left.\left(\frac{d^{2} V}{d x_{\alpha} d x_{\beta}}\right)\right|_{r=0}
$$

Higher-order terms in the multipole expansion can generally be ignored. 
The most convenient principal-axis system for the quadrupolar interaction will be the one in which the electric-field gradient $(\mathrm{EFG})$ is diagonal. It will then have three components, but due to Laplace's equation

$$
\nabla \mathrm{V}=0=\mathrm{V}_{\mathrm{XX}}+\mathrm{V}_{\mathrm{YY}}+\mathrm{V}_{\mathrm{ZZ}}
$$

only two independent parameters can be defined. These parameters are the strength of the EFG in units of electric charge

$$
\delta^{\mathrm{Q}}=\mathrm{eq}=\mathrm{V}_{\mathrm{zz}}
$$

and a parameter expressing the deviation of the electric field from cylindrical symmetry

$$
\eta^{\mathrm{Q}}=\frac{\mathrm{V}_{\mathrm{YY}}-\mathrm{V}_{\mathrm{XX}}}{\mathrm{V}_{\mathrm{ZZ}}}
$$

where $\left|V_{Z Z}\right| \geq\left|V_{Y Y}\right| \geq\left|V_{X X}\right|$.

It will prove convenient to rewrite the quadrupole-moment tensor as

$$
\mathrm{Q}_{\alpha \beta}=3 \mathrm{Q}_{\alpha \beta}{ }^{\prime}-\delta_{\alpha \beta} \sum_{\gamma=1}^{3} \mathrm{Q}_{\gamma \gamma}{ }^{\prime}
$$

which makes it traceless. Substituting Equations (1.75), (1.76), and (1.80) into Equation (1.74) and making use of Equation (1.77) gives

$$
E=\frac{1}{6} \sum_{\alpha=1}^{3} \sum_{\alpha=1}^{3} Q_{\alpha \beta} V_{\alpha \beta} .
$$

So far, no quantum-mechanical aspects have been introduced. However, nuclear spin is intrinsically quantum-mechanical; it is, therefore, necessary to invoke a correspondence principle to rewrite the moment tensor in terms of spin angular momentum operators.

According to the Wigner-Eckhart theorem, ${ }^{26}$ all matrix elements of a tensor of a given rank, $\mathrm{k}$, are proportional to each other 


$$
\left\langle\varepsilon, \mathrm{I}, \mathrm{m}\left|\mathrm{T}_{\mathrm{kn}}\right| \varepsilon, \mathrm{I}^{\prime}, \mathrm{m}^{\prime}\right\rangle=\mathrm{C}\left(\mathrm{I}, \mathrm{I}^{\prime}, \mathrm{m}, \mathrm{m}^{\prime}, \mathrm{k}, \mathrm{n}\right)\left\langle\varepsilon, \mathrm{I}\left|\mathrm{T}_{\mathrm{k}}\right| \varepsilon, \mathrm{I}^{\prime}\right\rangle
$$

where $\mathrm{n}$ is a tensor component; $\varepsilon$ is a generic quantum number; and $\mathrm{C}$ is a Clebsch-Gordan coefficient that is independent of the nature of the interaction. The Wigner-Eckhart theorem implies that the components of two irreducible tensors of the same rank will be related by a constant of proportionality

$$
\left\langle\varepsilon, \mathrm{I}, \mathrm{m}\left|\mathrm{Q}_{\mathrm{kn}}\right| \varepsilon, \mathrm{I}^{\prime}, \mathrm{m}^{\prime}\right\rangle=\left\{\frac{\left\langle\varepsilon, \mathrm{I}\left|\mathrm{Q}_{\mathrm{k}}\right| \varepsilon, \mathrm{I}^{\prime}\right\rangle}{\left\langle\varepsilon, \mathrm{II} \mathrm{T}_{\mathrm{k}} \mid \varepsilon, \mathrm{I}^{\prime}\right\rangle}\right\}\left\langle\varepsilon, \mathrm{I}, \mathrm{m}\left|\mathrm{T}_{\mathrm{kn}}\right| \varepsilon, \mathrm{I}^{\prime}, \mathrm{m}^{\prime}\right\rangle
$$

where the constant of proportionality is given in curly brackets: Applying this to Equation (1.80) gives

$$
\left\langle\varepsilon, \mathrm{I}, \mathrm{m}\left|\mathrm{Q}_{\alpha \beta}\right| \varepsilon, \mathrm{I}^{\prime}, \mathrm{m}^{\prime}\right\rangle=\zeta\left\langle\varepsilon, \mathrm{I}, \mathrm{m}\left|\left(3 \frac{\left(\mathrm{I}_{\alpha} \mathrm{I}_{\beta}+\mathrm{I}_{\beta} \mathrm{I}_{\alpha}\right)}{2}-\delta_{\alpha \beta} \overrightarrow{\mathrm{I}} \cdot \overrightarrow{\mathrm{I}}\right)\right| \varepsilon, \mathrm{I}^{\prime}, \mathrm{m}^{\prime}\right\rangle
$$

where the quantum-mechanical operators have been symmetrized. ${ }^{5}$ Defining a quadrupole moment

$$
\mathrm{eQ}=\left\langle\varepsilon, \mathrm{I}, \mathrm{m}\left|\mathrm{Q}_{\mathrm{zz}}\right| \varepsilon, \mathrm{I}^{\prime}, \mathrm{m}^{\prime}\right\rangle
$$

and comparing with Equation (1.84) allows the constant of proportionality to be written as

$$
\zeta=\frac{\mathrm{eQ}}{\mathrm{I}(2 \mathrm{I}-1)}
$$

and the matrix elements of the quantum-mechanical Hamiltonian become

$$
\begin{array}{r}
\left\langle\mathrm{I}, \mathrm{m}\left|\mathcal{H}_{\mathrm{Q}}\right| \mathrm{I}, \mathrm{m}^{\prime}\right\rangle=\frac{\mathrm{eQ}}{6 \mathrm{I}(2 \mathrm{I}-1)} \sum_{\alpha=1 \beta=1}^{3} \sum_{\alpha=1}^{3}\langle\mathrm{I}, \mathrm{m}|\left(3 \frac{\left(\mathrm{I}_{\alpha} \mathrm{I}_{\beta}+\mathrm{I}_{\beta} \mathrm{I}_{\alpha}\right)}{2}\right. \\
\left.-\delta_{\alpha \beta} \overrightarrow{\mathrm{I}} \cdot \overrightarrow{\mathrm{I}}\right)\left|\mathrm{I}, \mathrm{m}^{\prime}\right\rangle \mathrm{V}_{\alpha \beta} .
\end{array}
$$


Using the Wigner-Eckhart theorem and the "triangle selection rule" for Clebsch-Gordan coefficients, ${ }^{1,2}$ it is also possible to demonstrate that only nuclei with a spin-quantum number greater than one-half will have a quadrupole moment. Over two-thirds of the NMR-active nuclides in the periodic table satisfy this condition.

As is the case for the other NMR Hamiltonians, it is convenient to express the Hamiltonian in terms of products of spherical tensor operators rather than Cartesian ones

$$
\mathscr{H}_{\mathrm{Q}}=\frac{\mathrm{e}^{2} \mathrm{qQ}}{2 \mathrm{I}(2 \mathrm{I}-1)} \sum_{1=0,2} \sum_{\mathrm{m}=-2}^{2}(-1)^{\mathrm{m}_{\mathrm{m}}} \mathrm{R}_{\mathrm{l},-\mathrm{m}}^{\mathrm{Q}} \mathrm{T}_{\mathrm{l}, \mathrm{m}}^{\mathrm{Q}}
$$

where

$$
\begin{gathered}
\rho_{0,0}^{Q}=0 \\
\rho_{2,0}^{Q}=\sqrt{\frac{3}{2}} \\
\rho_{2, \pm 1}^{Q}=0 \\
\rho_{2, \pm 2}^{Q}=\frac{1}{2} \eta^{Q}
\end{gathered}
$$

and

$$
\begin{gathered}
\mathrm{T}_{0,0}^{\mathrm{Q}}=\mathrm{I}^{2} \\
\mathrm{~T}_{2,0}^{\mathrm{Q}}=\frac{1}{\sqrt{6}}\left[3 \mathrm{I}_{\mathrm{z}}^{2}-\mathrm{I}^{2}\right] \\
\mathrm{T}_{2, \pm 1}^{\mathrm{Q}}=\mp \frac{1}{2}\left[\mathrm{I}_{ \pm} \mathrm{I}_{\mathrm{z}}+\mathrm{I}_{\mathrm{z}} \mathrm{I}_{ \pm}\right] \\
\mathrm{T}_{2, \pm 2}^{\mathrm{Q}}=\frac{1}{2} \mathrm{I}_{ \pm}^{2} .
\end{gathered}
$$

Note that $\delta^{Q}$ (see Equation (1.78)) has been included in the constant in front of the sum in Equation (1.88) rather than in the $\rho_{2, n}^{Q}$ terms. 
Because of the size of the quadrupolar interaction, it is necessary to consider not only first-order perturbation terms (i.e.- those arising from the secular approximation) when transforming to the rotating frame but also second-order terms. To derive these, we will take the approach of Goldman et al. ${ }^{28}$ which utilizes an operator form of perturbation theory. Such an approach avoids the necessity of introducing correction terms as must be done when using coherent Average-Hamiltonian Theory. ${ }^{7,10}$

The operator form of static perturbation theory is simply a generalization of the standard non-degenerate perturbation theory described in Section 1.1.4. The energy corrections are projected onto the basis of eigenvectors of the zeroth-order Hamiltonian which leads to an operator version of Equation (1.44)

$$
\mathrm{D}=\mathcal{H}_{0}+\mathrm{D}^{(1)}+\mathrm{D}^{(2)}+\ldots
$$

where

$$
D^{(n)}=\sum_{j}|j\rangle E_{j}^{(n)}\langle j|
$$

and the $E_{j}^{(n)}$ are given by Equations (1.45) and (1.46). The advantage of this method over traditional perturbation calculations of individual shifts ${ }^{11}$ is that one can obtain an analytical form for the Hamiltonian which can be used to describe the evolution of any transition. This will be particularly useful for the simulations of Chapter 3 .

For the quadrupolar interaction, the first order quadrupolar Hamiltonian can easily be shown to be (in energy units)

$$
\tilde{\mathcal{H}}_{\mathrm{Q}}^{(1)}=\hbar\left(\frac{\omega_{\mathrm{Q}}}{3}\right) \mathrm{R}_{2,0}^{\mathrm{Q}} \mathrm{T}_{2,0}^{\mathrm{Q}}
$$

where $\omega_{Q}$ is the quadrupolar coupling constant

$$
\omega_{Q}=\frac{3 e^{2} q Q}{2 I(2 I-1) \hbar}=\frac{6 \pi}{2 I(2 I-1)} C_{q c c} .
$$


Equation (1.93) is identical with what would be obtained by making the secular approximation. The second-order contribution is calculated from Equations (1.46) and (1.92) as follows

$$
\tilde{\mathcal{H}}_{\mathrm{Q}}^{(2)}=\mathrm{D}^{(2)}=\sum_{\mathrm{j}} \sum_{\mathrm{k} \neq \mathrm{j}} \frac{|\mathrm{j}\rangle\left\langle\mathrm{j}\left|\mathscr{H}_{\mathrm{Q}}\right| \mathrm{k}\right\rangle\left\langle\mathrm{k}\left|\mathscr{H}_{\mathrm{Q}}\right| \mathrm{j}\right\rangle\langle\mathrm{j}|}{\mathrm{E}_{\mathrm{j}}^{(0)}-\mathrm{E}_{\mathrm{k}}^{(0)}}
$$

Defining $\mathrm{m} \equiv \mathrm{j}-\mathrm{k}$ gives

$$
\tilde{\mathcal{H}}_{\mathrm{Q}}^{(2)}=\frac{\hbar}{\omega_{L}}\left(\frac{\omega_{\mathrm{Q}}}{3}\right)^{2} \sum_{j} \sum_{m \neq 0} R_{2,-m}^{Q} R_{2, m}^{Q} \frac{|j\rangle\left\langle j\left|T_{2, m}^{Q}\right| j-m\right\rangle\left\langle j-m\left|T_{2,-m}^{Q}\right| j\right\rangle\langle j|}{\dot{m}} .
$$

But

$$
|j-m\rangle\left\langle j-m\left|=1-\sum_{n \neq j-m}\right| n\right\rangle\langle n| .
$$

Substitution leads to

$$
\tilde{\mathcal{H}}_{\mathrm{Q}}^{(2)}=\frac{\hbar \omega_{\mathrm{Q}}^{2}}{9 \omega_{\mathrm{L}}} \sum_{\mathrm{j}} \sum_{\mathrm{m} \neq 0} \mathrm{R}_{2,-\mathrm{m}}^{\mathrm{Q}} \mathrm{R}_{2, \mathrm{~m}}^{\mathrm{Q}} \frac{|\mathrm{j}\rangle\left\langle\mathrm{j}\left|\mathrm{T}_{2, \mathrm{~m}}^{\mathrm{Q}} \mathrm{T}_{2,-\mathrm{m}}^{\mathrm{Q}}\right| \mathrm{j}\right\rangle\langle\mathrm{j}|}{\mathrm{m}} .
$$

Because the term $T_{2, m}^{Q} T_{2,-m}^{Q}$ commutes with the projection operator, Equation (1.98) becomes

$$
\tilde{\mathcal{H}}_{\mathrm{Q}}^{(2)}=\frac{\hbar \omega_{\mathrm{Q}}^{2}}{9 \omega_{\mathrm{L}}} \sum_{\mathrm{m} \neq 0} \mathrm{R}_{2,-\mathrm{m}}^{\mathrm{Q}} \mathrm{R}_{2, \mathrm{~m}}^{\mathrm{Q}} \frac{\mathrm{T}_{2, \mathrm{~m}}^{\mathrm{Q}} \mathrm{T}_{2,-\mathrm{m}}^{\mathrm{Q}}}{\mathrm{m}}
$$

Explicitly summing and regrouping gives

$$
\tilde{\mathcal{H}}_{\mathrm{Q}}^{(2)}=\frac{\hbar \omega_{\mathrm{Q}}^{2}}{18 \omega_{\mathrm{L}}}\left\{2 \mathrm{R}_{2,1}^{\mathrm{Q}} \mathrm{R}_{2,-1}^{\mathrm{Q}}\left[\mathrm{T}_{2,1}^{\mathrm{Q}}, \mathrm{T}_{2,-1}^{\mathrm{Q}}\right]+\mathrm{R}_{2,2}^{\mathrm{Q}} \mathrm{R}_{2,-2}^{\mathrm{Q}}\left[\mathrm{T}_{2,2}^{\mathrm{Q}}, \mathrm{T}_{2,-2}^{\mathrm{Q}}\right]\right\}
$$

where $^{10}$ 


$$
\begin{aligned}
& {\left[\mathrm{T}_{2,1}^{\mathrm{Q}}, \mathrm{T}_{2,-1}^{\mathrm{Q}}\right]=\frac{1}{2}\left(4 \mathrm{I}^{2}-8 \mathrm{I}_{\mathrm{z}}^{2}-1\right) \mathrm{I}_{\mathrm{z}}} \\
& {\left[\mathrm{T}_{2,2}^{\mathrm{Q}}, \mathrm{T}_{2,-2}^{\mathrm{Q}}\right]=\left(2 \mathrm{I}^{2}-2 \mathrm{I}_{\mathrm{z}}^{2}-1\right) \mathrm{I}_{\mathrm{z}}}
\end{aligned}
$$

Both the first- and second- order terms are orientation dependent.

Figure 1.2a shows the energy-level diagram for a single spin- $5 / 2$ nucleus subject to the effects of the quadrupolar interaction to both first- and second-order. Note that to firstorder, the frequency of the $(-\mathrm{m} \leftrightarrow \mathrm{m})$ transitions (including the directly ofservable central transition) are unaffected (due to the fact that $T_{2,0}^{Q}$ only depends on even powers of $I_{z}$ ). This is generally the case for nuclei with odd-half-integer spin-quantum numbers. Inclusion of the second-order terms will influence all of the transitions. In a powdered solid sample, the central transition will be anisotropically broadened with a lineshape that depends on the values of $\mathrm{C}_{\mathrm{qcc}}$ and $\eta$, and an additional contribution to the isotropic shift will also be introduced (see Section 1.4.2). It is possible to extract values of the quadrupolar parameters by performing lineshape simulations; these parameters can then be related to bond geometries..$^{29,30,31}$ Further discussion of the nature of the central transition lineshape can be found in Chapter 6.

In the experiments described in this thesis, the strength of the applied rf field will not be strong enough to excite all allowed transitions simultaneously. However, selective excitation of the central transition $(\mathrm{m}=-1 / 2 \leftrightarrow \mathrm{m}=1 / 2)$ is possible if a relatively weak $\mathrm{rf}$ field $\left|\omega_{1 \mathrm{I}}\right| \ll\left|\omega_{\mathrm{Q}}\right|$ is applied near resonance. ${ }^{27}$ In such a case, the full $I_{x}$ and $I_{y}$ operators will not enter into Equation (1.58). Instead, one can assume that only the two-by-two submatrix between the $m=-1 / 2$ and $m=+1 / 2$ levels will be relevant. From Equations (1.50) and (1.51), one can see that this fictitious spin-1/2 operator ${ }^{4}$ will have an additional factor relative to the true spin-1/2 case. For a pulse applied along the $+y$ axis, the operator in the $(-1 / 2,+1 / 2)$ submanifold will be

$$
\left(I+\frac{1}{2}\right) \cdot\left[\begin{array}{cc}
0 & \frac{-i}{2} \\
\frac{i}{2} & 0
\end{array}\right] \text {. }
$$



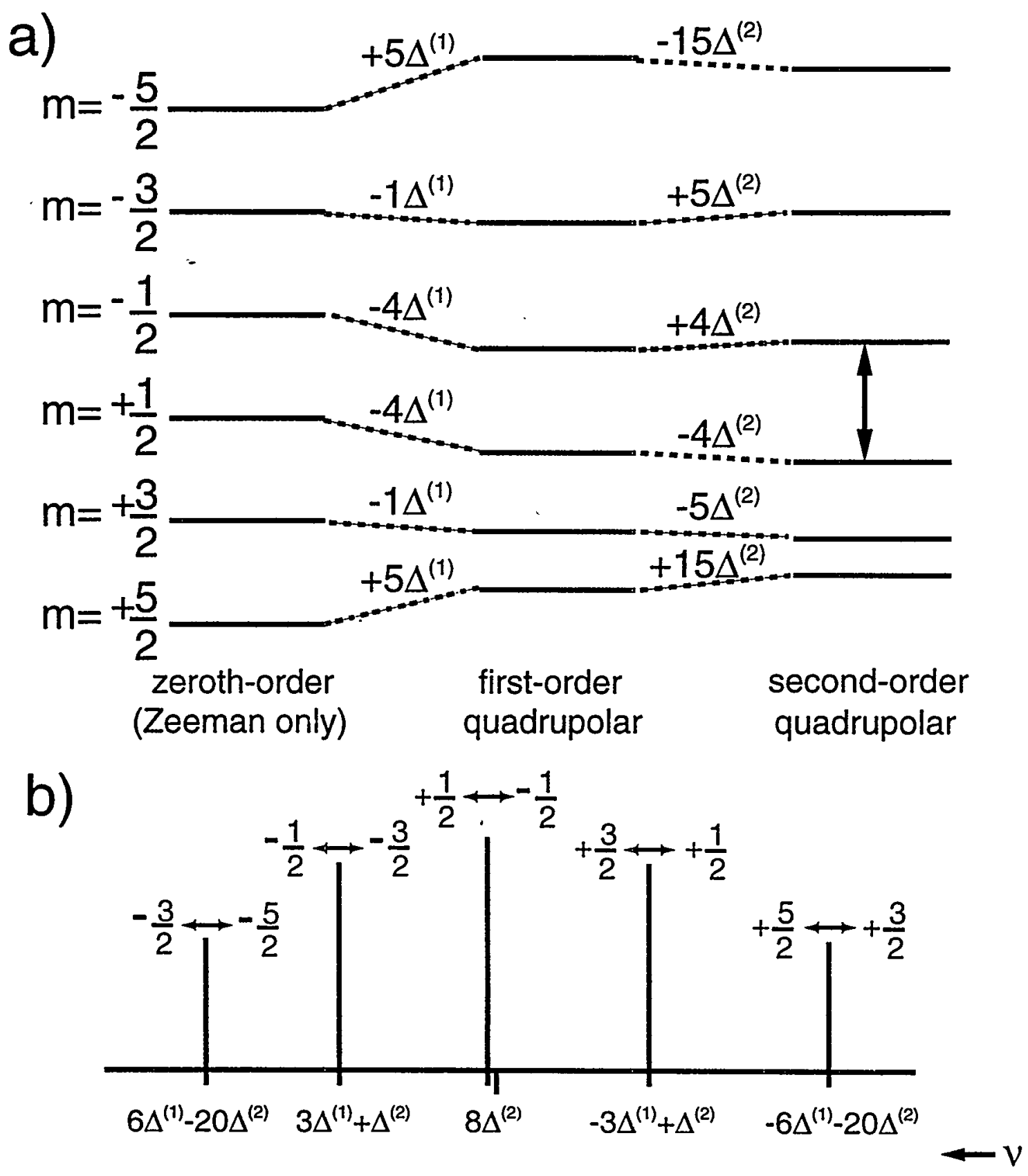

Figure 1.2 - (a) Energy level diagram for a single crystallite $\left(\eta^{\mathrm{Q}}=0^{\circ}, \beta^{\mathrm{Q}}=45^{\circ}\right)$ of a spin-5/2 nucleus in a static magnetic field. The effects of the first and second-order contributions to the quadrupolar Hamiltonian are depicted. Note that the frequencies of all $(-\mathrm{m} \leftrightarrow \mathrm{m})$ transitions are unaffected to first order. (b) The five allowed transitions of a spin-5/2 nucleus subject to both first- and second-order quadrupolar effects. Relative intensities of the five lines for non-selective excitation are 5:8:9:8:5.27 
The factor $(I+1 / 2)$ leads to an increased nutation frequency. Thus, the rf-field strength required to rotate the central transition by $90^{\circ}$ is $1 /(\mathrm{I}+1 / 2)$ of that required for a spin-1/2 nucleus (or for a quadrupole in a liquid in which nonselective excitation is easily achievable). ${ }^{27}$ This will be particularly important for the cross-polarization experiments of Chapter 4. A more rigorous derivation of this resul: may be found in the thesis of J. H. Baltisberger. ${ }^{11}$

\subsubsection{Other Interactions in NMR}

Other interactions can also potentially affect solid-state NMR spectra. One of these is an indirect dipolar-coupling mechanism which involves bonding electrons. It is known as the scalar interaction and is characterized by a coupling constant, J. Although important in solution-state spectroscopy, the J-coupling is generally neglected in solids since it is typically much smaller than the direct dipolar coupling. Its effects can sometimes be observed in highly-crystalline samples, however. ${ }^{32,33,34,35.36}$ This thesis will not explicitly treat the J-coupling. Other interactions such as spin-rotation coupling and the Knight shift in metals ${ }^{5}$ are also beyond the scope of this thesis.

So far all of the interactions in Sections 1.2.3 - 1.2.5 have been treated as separate, additive perturbations. However, there are also potentially second-order cross terms between the different interactions. ${ }^{27}$ The most significant of these would involve the quadrupolar interaction since it is the largest in the systems studied in this thesis. Such a cross term would lead to frequency shift in the spectrum of the spin-1/2 nucleus that is proportional to

$$
\frac{\omega_{D} \omega_{Q}}{\omega_{L}}
$$

in $\mathrm{Hz}$. Here $\omega_{\mathrm{D}}$ is the frequency of the dipolar coupling between the spins; $\omega_{\mathrm{Q}}$ is the quadrupolar frequency (see Equation (1.94)); and $\omega_{\mathrm{L}}$ is the Larmor frequency of the quadrupolar nucleus. For the dipolar couplings in the system studied in this thesis (see Chapter 3), such a term would be on the order of $5 \mathrm{~Hz}$ and is, therefore, negligible. 


\subsection{Powder Spectra}

So far this chapter has primarily been concerned with the orientation of a single crystallite. In solid-state NMR, however, powdered samples are often used. These powders are composed of many different crystallites, each of which is oriented differently with respect to the static magnetic field. This leads to broad powder patterns for each type of interaction (e.g. - CSA, dipolar, quadrupolar). Examples of these are shown in Figures $1.4 \mathrm{a}$ and $1.5 \mathrm{a}$.

To simulate the lineshapes of powder samples, it is necessary to sum over many different crystallite orientations. Conceptually, the most straightforward approach would be to use equidistant points on a sphere; in practice, however, this is somewhat inefficient and requires a large number of step sizes. ${ }^{11}$ Alternative methods of powder averaging have been proposed; ${ }^{37,38}$ the method used in this thesis is based on an algorithm described by Cheng et al., ${ }^{38}$ which traces out a spiral on a sphere. For a given number of points, this algorithm has been shown to be more accurate than averaging over "random" orientations. Using Cheng's method, the Euler angles $(\alpha, \beta, \gamma)$ are chosen according to

$$
\begin{gathered}
\alpha=\frac{360^{\circ} \cdot \bmod _{N}\left\{n \cdot v_{2}\right\}}{N}, \\
\beta=\frac{180^{\circ} \cdot n}{N},
\end{gathered}
$$

and

$$
\gamma=\frac{360^{\circ} \cdot \bmod _{N}\left\{\mathrm{n} \cdot \mathrm{v}_{3}\right\}}{\mathrm{N}}
$$

where $\mathrm{N}$ is the total number of orientations in the powder average; $\mathrm{n}$ is an integer ranging from 1 to $\mathrm{N}$; and $\mathrm{v}_{2}$ and $\mathrm{v}_{3}$ are tabulated in Tables 1.2 and 1.3. Note that different values of are $v_{2}$ are optimal depending on whether one averages over three angles (for an arbitrary rotation) or over two angles (e.g. - when cylindrical symmetry makes one of the Euler angles irrelevant). 


\begin{tabular}{|c||c|c|c|c|c|c|c|}
\hline $\mathbf{N}$ & $\mathbf{5 0}$ & $\mathbf{1 0 0}$ & $\mathbf{1 4 4}$ & $\mathbf{2 0 0}$ & $\mathbf{3 0 0}$ & $\mathbf{5 3 8}$ & $\mathbf{1 1 5 4}$ \\
\hline \hline $\mathbf{v}_{\mathbf{2}}$ & $\mathbf{7}$ & 27 & 11 & 29 & 37 & 55 & 107 \\
\hline $\mathbf{v}_{\mathbf{3}}$ & 11 & 41 & 53 & $\mathbf{7 9}$ & 61 & 229 & 271 \\
\hline
\end{tabular}

Table 1.2 - Table of parameters for use with Equations (1.105), (1.106), and (1.107) to determine suitable Euler angles $(\alpha, \beta, \gamma)$ for powder averaging in the general case. ${ }^{38}$

\begin{tabular}{|c||c|c|c|c|c|c|c|c|c|c|c|}
\hline N & $\mathbf{1 4 4}$ & $\mathbf{2 3 3}$ & $\mathbf{3 7 7}$ & $\mathbf{6 1 6}$ & $\mathbf{9 8 7}$ & $\mathbf{1 5 9 7}$ & $\mathbf{2 5 8 4}$ & $\mathbf{4 1 8 1}$ & $\mathbf{6 7 6 5}$ & $\mathbf{1 0 9 4 6}$ & $\mathbf{1 7 7 1 1}$ \\
\hline \hline $\mathrm{v}_{\mathbf{2}}$ & 55 & 89 & 144 & 233 & 377 & 616 & 987 & 1597 & 2584 & 4181 & 6765 \\
\hline
\end{tabular}

Table 1.3 - Table of parameters for use with Equations (1.105) and (1.106) to determine suitable Euler angles $(\alpha, \beta)$ for powder averaging when the symmetry of the problem is such that only two angles are required.

\subsection{Rotating Samples}

While the broad static powder patterns contain information about the environment of a given nuclear spin, they lead to poor resolution. Spectra of samples where the nuclei are subject to more than one interaction or where multiple sites are present quickly become uninterpretable. Fortunately, considerable improvement in resolution can be achieved if the sample is rapidly rotated about an axis that is not aligned with the static magnetic field. To understand how an external spatial reorientation can affect the spectra of internal interactions, it is necessary to use Wigner rotation matrices.

\subsubsection{Wigner Rotation Matrices Revisited}

The orientation dependence of a rotating sample can be calculated by performing two consecutive sets of Euler angle rotations as shown in Figure 1.3. The first set describes the orientation of an individual crystallite relative to the rotor frame, and the second set describes the orientation of the rotor relative to the $\mathrm{B}_{0}$ field. Mathematically, this can be expressed by applying Equation (1.35) in a nested manner

$$
R_{1, m}=\sum_{n=-1}^{1} \sum_{m^{\prime}=-1}^{1} D_{n, m}^{(1)}\left(\omega_{r} t+\phi_{r}, \theta, 0\right) D_{m^{\prime}, n}^{(1)}(\alpha, \beta, \gamma) \rho_{1, m^{\prime}}
$$


where $\theta$ is the angle between the rotor axis and the static magnetic field, $\omega_{\mathrm{r}}$ is the rate of rotation, and $\phi_{\mathrm{r}}$ is the initial phase of the rotor (which for simplicity we will set equal to zero in the following sections). Using Equation (1.36) and separating the $\mathrm{n}=0$ term gives

$$
\begin{aligned}
& \mathrm{R}_{\mathrm{l}, \mathrm{m}}=\mathrm{d}_{0, \mathrm{~m}}^{(1)}(\theta) \sum_{\mathrm{m}^{\prime}=-1}^{1} \mathrm{D}_{\mathrm{m}^{\prime}, 0}^{(1)}(\alpha, \beta, \gamma) \rho_{1, \mathrm{~m}^{\prime}} \\
& +\sum_{\substack{n=-1 \\
n \neq 0}}^{1} \sum_{m^{\prime}=-1}^{1} e^{-i n \omega_{r} t} d_{n, m}^{(1)}(\theta) D_{m^{\prime}, n}^{(1)}(\alpha, \beta, \gamma) \rho_{1, m^{\prime}}
\end{aligned}
$$

Note that the first term is time-independent. In the limit of very rapid rotation, the timedependent terms will average to zero, leaving only the time-independent term.
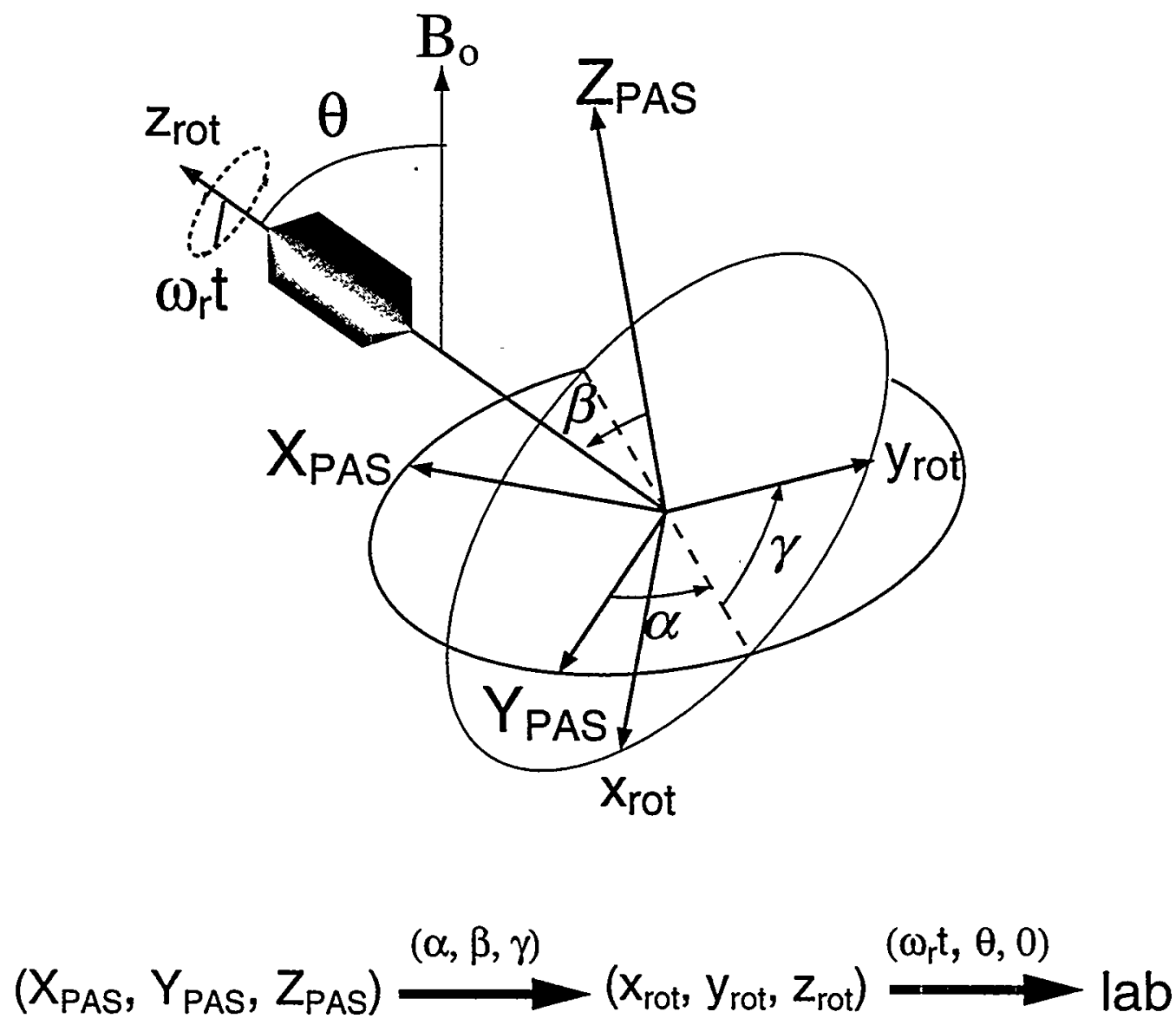

Figure 1.3 - Euler angle convention for a rotating sample. The first set of Euler angles $(\alpha, \beta, \gamma)$ describes the orientation of a given crystallite relative to the rotor axis, and the second set $\left(\omega_{\mathrm{r}} t, \theta, 0\right)$ describes the orientation of the rotor relative to the $B_{0}$ field. 
Several special cases are of interest. Recall that in the limit of large $\mathrm{B}_{0}$ fields, only spin tensors, $T_{1, m}$, with $m=0$ are retained in the Hamiltonian (secular approximation). This corresponds to retaining only spatial tensors, $R_{1, m}$, with $m=0$, due to the form of the NMR Hamiltonians (see Equations (1.60), (1.68), and (1.88)). Thus, the time-independent spatial terms in Equation (1.109) will be proportional to $\mathrm{d}_{0,0}^{(1)}(\theta)$. For $\mathrm{l}=2$ and $\theta=54.74^{\circ}$, $\mathrm{d}_{0,0}^{(1)}(\theta)$ equals zero; rapid spinning at $\theta=54.74^{\circ}$ (the "magic angle") will, therefore, eliminate the anisotropy in the $R_{2,0}$ terms. Note that the entire anisotropy of the chemical shielding and dipolar interactions is contained in such terms; the quadrupolar case is different and will be discussed in Section 1.4.2.

When the rate of spinning about the magic angle is less than or approximately equal to the breadth of the static lineshape, the time-dependent terms cannot be ignored. Considering the specific case of a spin subject to the chemical-shielding interaction, one can use Equations (1.12), (1.13), (1.66), and (1.109) to show that a crystallite will accumulate a phase given by

$$
\exp \left(-i \gamma B_{0} \int_{0}^{t}\left[\sigma_{i s o}+\sum_{\substack{n=-1 \\ n \neq 0}}^{1} \sum_{m^{\prime}=-2}^{1} e^{-i n \omega_{r} t} d_{n, 0}^{(2)}(\theta) D_{m^{\prime}, n^{\prime}}^{(2)}(\alpha, \beta, \gamma) \rho_{2, m^{\prime}}\right]\right)
$$

The time-dependent terms in Equation (1.110) can be regrouped into sines and cosines of $\omega_{\mathrm{r}} \mathrm{t}$ and $2 \omega_{\mathrm{r}} \mathrm{t}$. Using a property of Bessel functions

$$
e^{i z \sin \phi}=\sum_{k=-\infty}^{\infty} e^{i k \phi} J_{k}(z)
$$

one can show that the signal depends periodically on the rotation. ${ }^{39}$ When such a signal is Fourier transformed, a series of "sidebands" spaced at the rotor frequency will appear in the spectrum, and the intensities of the sidebands will contain information about the chemical-shielding parameters. ${ }^{40}$ Herzfeld and $B e r g e r^{40}$ have tabulated ratios of the sideband intensities to the centerband intensity for different values of the CSA parameters to allow them to be rapidly determined from a magic-angle spinning (MAS) spectrum; with modern computers, direct fitting of the spectrum can also be accomplished. 
Figure 1.4 shows simulations of spectra corresponding to the CSA interaction at various spinning speeds. Note that since the total integrated intensity of the spectrum is constant, rapid MAS not only improves resolution but also sensitivity. The spectrum in Figure $1.4 \mathrm{c}$ is of the type that can be used for Herzfeld-Berger analysis.

Another interesting angle is $\theta=0^{\circ}$. Using the definition of the reduced Wigner rotation matrix elements (Equation (1.37)), one can show that for $\theta=0^{\circ}$

$$
d_{n, m}^{(1)}\left(0^{\circ}\right)=\left\{\begin{array}{l}
0 \text { for } n \neq m \\
1 \text { for } n=m
\end{array} .\right.
$$

Since only $m=0$ terms are relevant in the secular approximation, it is easy to see (by using Equations (1.109) and (1.112)) that all time-dependent terms vanish for $\theta=0^{\circ}$. In fact, what remains is identical to the full, static powder pattern. Thus, spinning about an axis parallel to the $\mathrm{B}_{0}$ field is equivalent, from a theoretical point of view, to not spinning the sample at all. This can be viewed as a consequence of the $C_{\infty}$ symmetry induced by the $B_{0}$
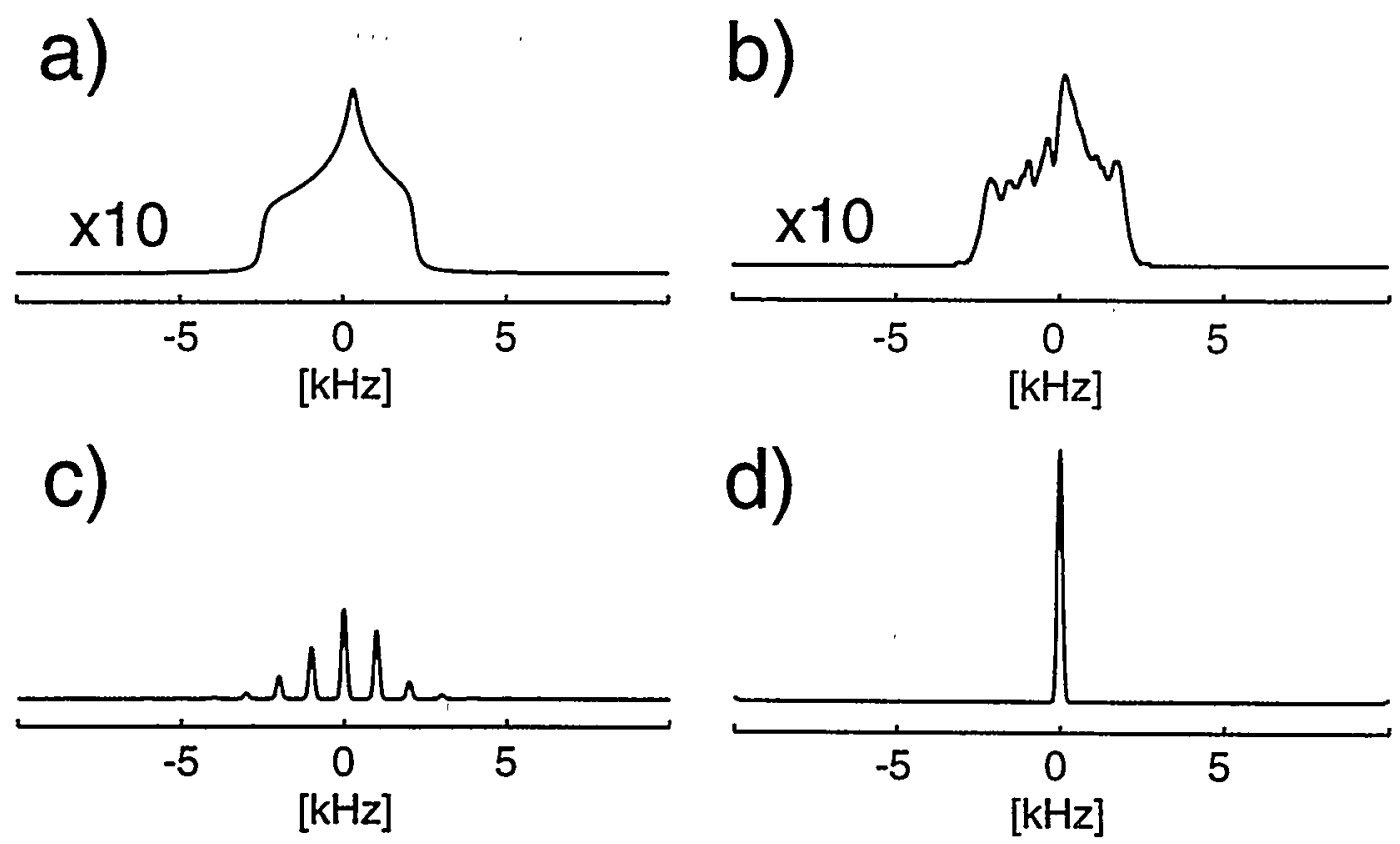

Figure 1.4 - Simulated CSA powder patterns for $\delta^{\mathrm{CS}}=-2.5 \mathrm{kHz}$ and $\eta^{\mathrm{CS}}=0.76$ at spinning speeds of (a) $0 \mathrm{~Hz}$, (b) $100 \mathrm{~Hz}$, (c) $1 \mathrm{kHz}$, and (d) $10 \mathrm{kHz}$. The total integrated intensity of all spectra are the same, but for clarity, the vertical axes of (a) and (b) have been scaled by a factor of 10. The rapid spinning spectrum in (d) has high sensitivity, but all information about the CSA parameters is lost. The spectrum in (c) represents a good compromise between sensitivity and information content. 
field. In practice, spinning about $0^{\circ}$ has the advantage of reducing the effects of magnetic field inhomogeneity, but the disadvantage of requiring specialized solid-state NMR probe technology.

\subsubsection{Effects of Sample Rotation on Quadrupolar Lineshapes}

For odd-half-integer quadrupolar nuclei, the situation is more complicated. This is due to the functional form of the second-order quadrupolar anisotropy, which, as can be seen from Equation (1.100), depends on products of second-rank spatial tensors. It is well known that the product of two commuting second-rank tensors can be written as a sum of zeroth-, second-, and fourth- rank tensors. ${ }^{41}$ By substituting Equation (1.108) into Equation (1.100), one can explicitly calculate the form of this anisotropy for a sample spun about an axis $\theta$ with respect to the static magnetic field. In this thesis, we will be interested in the frequencies of the $(+\mathrm{m} \leftrightarrow-\mathrm{m})$ transitions

$$
\omega_{+\mathrm{m} \leftrightarrow-\mathrm{m}}^{(2 \mathrm{Q})}=\left\langle\mathrm{I}, \mathrm{m}\left|\frac{\tilde{\mathcal{H}}_{\mathrm{Q}}^{(2)}}{\hbar}\right| \dot{\mathrm{I}}, \mathrm{m}\right\rangle-\left\langle\mathrm{I},-\mathrm{m}\left|\frac{\tilde{\mathcal{H}}_{\mathrm{Q}}^{(2)}}{\hbar}\right| \dot{\mathrm{I}},-\mathrm{m}\right\rangle
$$

Explicit calculation of $\omega_{+\mathrm{m} \leftrightarrow-\mathrm{m}}^{(2 \mathrm{Q})}$ is laborious even when sidebands are neglected and only time-independent terms retained. The steps needed to do this are outlined in the thesis of K. T. Mueller ${ }^{10}$ for the case $m=1 / 2$ and have since been generalized to other values of $\mathrm{m}^{42,43}$ We will present only the final result of this calculation (valid for the case of fast spinning relative to the static linewidth)

$$
\begin{aligned}
\omega_{+m \leftrightarrow-m}^{(2 \mathrm{Q})}=\frac{\mathrm{C}_{\mathrm{qcc}}^{2}}{\omega_{\mathrm{L}}}\left\{\mathrm{A}_{0} \mathrm{C}_{0}(\mathrm{I}, \mathrm{m})\right. & +\mathrm{A}_{2}\left(\alpha^{\mathrm{Q}}, \beta^{\mathrm{Q}}\right) \mathrm{C}_{2}(\mathrm{I}, \mathrm{m}) \mathrm{P}_{2}(\cos \theta) \\
& \left.+\mathrm{A}_{4}\left(\alpha^{\mathrm{Q}}, \beta^{\mathrm{Q}}\right) \mathrm{C}_{4}(\mathrm{I}, \mathrm{m}) \mathrm{P}_{4}(\cos \theta)\right\}
\end{aligned}
$$

where $\alpha^{\mathrm{Q}}$ and $\beta^{\mathrm{Q}}$ describe the orientation of a given crystallite relative to the rotor axis. Note that, as expected, the second-order quadrupolar anisotropy is the sum of zeroth-, second-, and fourth- rank terms (see Equations (1.38)-(1.41)). Equation (1.114) will be discussed in more detail in Chapter 6; for now what is important to notice is that in addition to the second-order Legendre polynomial, there is also a dependence on the 
fourth-order Legendre polynomial. Thus spinning at the magic angle only removes part of the quadrupolar anisotropy (see Figure 1.5). Experiments which can produce isotropic lines for odd-half-integer quadrupolar nuclei will be discussed in Chapter 6 .

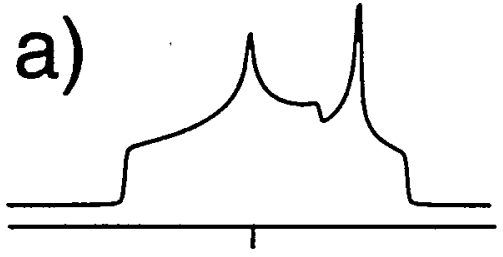

Frequency

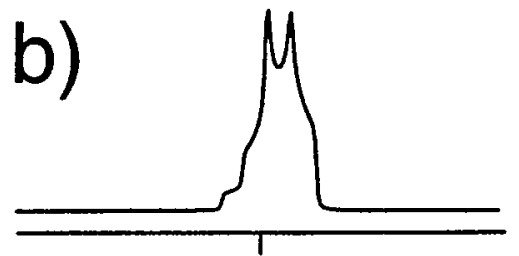

Frequency

Figure 1.5 - Schematic of second-order quadrupolar lineshapes for the central transition - (with $\eta=0.5$ ) in (a) a static sample and (b) a sample undergoing fast MAS. Removal of the $P_{2}(\cos \theta)$ term has significantly narrowed the lineshape but not eliminated its anisotropic character.

\subsection{Phase Cycling and Data Processing}

So far most of the discussions in this thesis have been concerned with the timedomain behavior of the NMR Hamiltonians. However, spectra are typically presented and analyzed in the frequency domain. The two domains can be related by a mathematical manipulation known as the Fourier transform. If $s(t)$ is the evolution of the transverse magnetization as a function of time, the frequency domain signal, $S(\omega)$, will be given by the complex Fourier transform of $s(t)^{4}$

$$
S(\omega)=\int_{-\infty}^{\infty} s(t) e^{-i \omega t} d t=\int_{-\infty}^{\infty} s(t)(\cos \omega t-i \sin \omega t) d t
$$

In NMR, one will often (though not always) record signals which are defined for $t \geq 0$ only. In such cases, the Fourier transform, $S(\omega)$, will contain both real (absorptive) and imaginary (dispersive) parts. The two most common Fourier transform pairs in NMR are summarized in Table 1.4. ${ }^{23}$ The discussion which follows in Section 1.5.2 will focus on lines which have a Lorentzian lineshape although the same principles apply to Gaussian lines as well. 


\begin{tabular}{|c|c|c|}
\hline Line Type & $s(t)$ & $S(\omega)$ \\
\hline \hline Lorentzian & $\mathrm{e}^{\mathrm{i} \Omega \mathrm{t}} \mathrm{e}^{-\mathrm{t} / \mathrm{T}_{2}}$ & $\frac{\mathrm{T}_{2}}{1+(\omega-\Omega)^{2}\left(\mathrm{~T}_{2}\right)^{2}}-\mathrm{i} \frac{(\omega-\Omega)\left(\mathrm{T}_{2}\right)^{2}}{1+(\omega-\Omega)^{2}\left(\mathrm{~T}_{2}\right)^{2}}=\mathrm{A}(\omega)-\mathrm{iD}(\omega)$ \\
\hline Gaussian & $\mathrm{e}^{\mathrm{i} \Omega \mathrm{t}} \mathrm{e}^{-\mathrm{t}^{2} /\left(\mathrm{T}_{2}\right)^{2}}$ & $\left(\sqrt{\pi} \mathrm{T}_{2}\right) \mathrm{e}^{\left.-i(\omega-\Omega)^{2}\left(\mathrm{~T}_{2}\right)^{2}\right\} / 4}$ \\
& & $\times\left\{\frac{1}{2}\left(1+\operatorname{erf}\left[\frac{\mathrm{i}(\omega-\Omega) \mathrm{T}_{2}}{2}\right]\right)\right\}$ \\
\hline
\end{tabular}

Table 1.4 - Complex Fourier transforms of Lorentzian and Gaussian decays which are defined for positive time values $(t \geq 0)$ only. This lack of symmetry about $t=0$ leads to dispersive components.

Useful theorems pertaining to Fourier transforms have been summarized in monographs on $\mathrm{NMR}^{4,44}$ and will not be reviewed here. Other important considerations such as digitization (dwell times, spectral width, resolution), zero filling, and apodization will also not be addressed in this thesis. Instead, the next two sections will focus on how to control the evolution of the signals that are observed and how to use such methods to obtain artifact-free, two-dimensional spectra.

\subsubsection{Coherence-Transfer Pathways}

In NMR, signals are typically recorded using quadrature detection, which corresponds to simultaneously observing two orthogonal components of the transverse magnetization. Such an acquisition scheme allows one to distinguish $\left(\omega_{L}+\Omega\right)$ from $\left(\omega_{L^{-}}\right.$ $\Omega$ ). If only one component were detected, those two frequencies could not be distinguished. Mathematically, the detected signal, also known as a free-induction decay (FID), can be written as ${ }^{11}$

$$
s(t)=\operatorname{Tr}\left\{\rho(t) e^{-t / T_{2}} I_{+} e^{i \phi_{R}}\right\}
$$

where $\phi_{R}$ is the phase of the receiver and $I_{+}$is the observable for quadrature detection. (In principle, the quadrature operator could be described instead by $I_{-}$, but we follow the convention of Ernst et al. ${ }^{4}$ ) 
From Equations (1.49), (1.54), (1.55), and (1.116), it is easy to see that only I. terms in the density matrix will produce observable signal. It is, therefore, necessary to apply pulses in such a way that all the frequencies of interest to the spectroscopist appear as prefactors of $I_{-}$. The way to do this is by coadding signals from experiments in which the phases of the rf pulses are varied in a systematic way.

To understand why such a phase cycling procedure works, it is useful to look the behavior of different coherences under rotations about different axes. In Section 1.1.1, a "coherence" was defined as an off-diagonal element of the density matrix. Coherences can be categorized by their order, or total change in magnetic quantum number. ${ }^{4}$ This is equivalent to characterizing them by their rate of precession about the $z$-axis since a coherence $\sigma_{p}$ of order $p$ acquires phase as follows ${ }^{4}$

$$
e^{-i \phi F_{z}} \sigma_{p} e^{i \phi F_{z}}=\sigma_{p} e^{-i p \phi}
$$

where $F_{z}$ is the total $z$ magnetization. Note that evolution about the $z$-axis (i.e. - free precession) does not change the order of a coherence.

Rotation about the $\mathrm{x}$ or $\mathrm{y}$ axis (as induced by an rf pulse) can change the order of a coherence as can be calculated from the equations in Sections 1.2.1 and 1.2.2. For instance, a $90^{\circ}$ pulse applied to the equilibrium magnetization will change the order from $\mathrm{p}=0$ to a linear combination of $\mathrm{p}=+1$ and $\mathrm{p}=-1$. A $180^{\circ}$ pulse will change $\mathrm{p}=+1$ to $\mathrm{p}=-1$, etc. Following Ernst et al. ${ }^{4}$, we can write the effects of a generalized rf pulse as

$$
\mathrm{U}_{\mathrm{rf}} \sigma_{\mathrm{p}} \mathrm{U}_{\mathrm{rf}}^{-1}=\sum_{\mathrm{p}^{\prime}} \sigma_{\mathrm{p}^{\prime}}
$$

where $U_{\text {rf }}$ represents the action of the pulse. Phase shifting that pulse by $\phi$ and rearranging gives 


$$
\begin{aligned}
e^{-i \phi F_{z}} U_{r f} e^{i \phi F_{z}} \sigma_{p} e^{-i \phi F_{z}} U_{r f}^{-1} e^{i \phi F_{z}} & =e^{-i \phi F_{z}} U_{r f}\left(\sigma_{p} e^{i p \phi}\right) U_{r f}^{-1} e^{i \phi F_{z}} \\
& =e^{-i \phi F_{z}}\left(U_{r f} \sigma_{p} U_{r f}^{-1}\right) e^{i \phi F_{z}} e^{i p \phi} \\
& =\sum_{p^{\prime}} e^{-i \phi F_{z}} \sigma_{p^{\prime}} e^{i \phi F_{z}} e^{i p \phi} \\
& =\sum_{p^{\prime}} \sigma_{p^{\prime}} e^{-i(\Delta p) \phi}
\end{aligned}
$$

where $\Delta \mathrm{p}=\mathrm{p}^{\prime}-\mathrm{p}$.

It is now easy to see that by summing experiments with different phase shifts, the experimenter can control which coherences are retained and which are eliminated. Detailed information on how to do this can be found elsewhere $e^{4,11,45}$ and only a few key points will be summarized below.

The first point is that when one increments the phase of a pulse from experiment to experiment by

$$
\Delta \phi=2 \pi / \mathrm{N}
$$

one will select every $\mathrm{N}$-th value of $\Delta \mathrm{p} .^{4}$ This rule determines the minimum length of phase cycle necessary to eliminate a certain coherence. For instance, suppose one wants to select the +1 quantum coherence but eliminate the -1 quantum coherence. The minimum phase cycle that achieves this would be a cycle with steps of $2 \pi / 3$ or $120^{\circ}$. Of course, smaller steps (such as $90^{\circ}$ phase increments) would also eliminate the -1 quantum coherence although this means that a longer phase cycle would be necessary. It will often be desirable to retain more than one coherence at a given stage, particularly if one wants to obtain pure absorption-phase lineshapes (see Section 1.5.2). This can easily be done using Equation (1.120); a phase cycle with steps of $2 \pi / 2$ or $180^{\circ}$ can be used to retain $p= \pm 1$ while eliminating $p=0, \pm 2$. Of course, some higher order coherences $(p= \pm 3, \pm 5)$ will also be retained in principle. In practice, however, high-order coherences can often be ignored since (1) they may have a low or zero probability of existing (e.g. - an isolated system of 
two spin-1/2 nuclei can at most have $\mathrm{p}= \pm 2$; even in large spin systems the highest possible coherences will be rare) or (2) later steps of the phase cycle will prevent them from contributing to the final observed signal.

To detect the $p=-1$ coherence, the phase of the receiver is cycled according to

$$
\phi_{R}=-\sum_{i}(\Delta \mathrm{p})_{i} \phi_{i}
$$

where $(\Delta \mathrm{p})_{\mathrm{i}}$ is the desired change in coherence induced by the $\mathrm{i}$-th pulse. By combining Equations (1.120) and (1.121), it is possible to design a pulse sequence in which different coherences are specifically retained during different time periods of the experiment. Such a sequence can be graphically represented as a "coherence transfer pathway." Examples of these will be seen throughout this thesis (see, for instance, Figures 5.3 and 6.10) where thick lines are used to indicate which coherences contribute to the final signal. Note that a common shorthand notation for pulse phases that are multiples of $90^{\circ}$ is $0=0^{\circ}, 1=90^{\circ}$, $2=180^{\circ}$, and $3=270^{\circ}$.

Phase cycling can be used to removed hardware-induced artifacts. The CYCLOPS cycle ${ }^{46}$ involves shifting the phase of the last pulse along with the receiver through all four spectrometer channels so that the effects of receiver imbalance are removed. Another common technique, spin-temperature alternation ${ }^{47}$ reverses the sense of precession (i.e.the direction of the magnetization vector) of the I spins relative to the $B_{1 I}$ spin-lock field from scan to scan by shifting the phase of the excitation pulse by $180^{\circ}$. If the I spin magnetization is transferred to the $S$ spins via cross polarization, the sense of precession of the $S$ spins about the $B_{1 S}$ field will also alternate between scans. ${ }^{23}$ Consequently, crosspolarized S-spin signal can be differentiated from directly excited S-spin signal, which will always precess in the same direction for a given $B_{1 S}$ field. Spin-temperature alternation is frequently used in cross-polarization experiments to suppress direct signals.

Another important use of phase cycles is for obtaining pure absorption-phase lineshapes in two-dimensional spectra. It is to that topic that we shall now turn. 


\subsubsection{Pure-Phase Two-Dimensional Spectra}

From Table 1.4, we see that the Fourier transform of an exponentially decaying, complex signal has both absorptive and dispersive components. For maximal resolution, however, it is desirable to have only absorptive peak shapes. In the one-dimensional case, it is always possible to obtain a spectrum which is purely absorptive in one channel of the detector and purely dispersive in the other. (Often this is done in practice by applying a phase correction after collection of arbitrarily phased data.) This is not as easily done in two-dimensional case, however. A generic two-dimensional FD can be written as

$$
s\left(t_{1}, t_{2}\right)=e^{i \Omega_{1} t_{1}} e^{i \varphi_{1}} e^{-t_{1} / T_{2}} e^{i \Omega_{2} t_{2}} e^{i \varphi_{2}} e^{-t_{2} / T_{2}}
$$

where $\varphi_{1}$ and $\varphi_{2}$ are arbitrary phase factors. Since it is always possible to phase correct the data, we can set these phase factors to zero without any loss of generality. Performing a Fourier transform over each time variable gives (see Table 1.4) (.11 $^{4}$

$$
\begin{aligned}
\mathrm{S}\left(\omega_{1}, \omega_{2}\right)= & \left(\mathrm{A}\left(\omega_{1}\right)-\mathrm{i} \mathrm{D}\left(\omega_{1}\right)\right)\left(\mathrm{A}\left(\omega_{2}\right)-\mathrm{iD}\left(\omega_{2}\right)\right) \\
=[ & \left.\mathrm{A}\left(\omega_{1}\right) \mathrm{A}\left(\omega_{2}\right)-\mathrm{D}\left(\omega_{1}\right) \mathrm{D}\left(\omega_{2}\right)\right] \\
& -\mathrm{i}\left[\mathrm{A}\left(\omega_{1}\right) \mathrm{D}\left(\omega_{2}\right)+\mathrm{D}\left(\omega_{1}\right) \mathrm{A}\left(\omega_{2}\right)\right]
\end{aligned}
$$

Clearly, neither channel is purely absorptive. The presence of a dispersive term in the real channel leads to "phase-twist" lineshapes with negative intensity in the wings.

To obtain purely absorptive lineshapes, it is necessary to use a more complicated acquisition scheme. ${ }^{48}$ This can be done by the methods of time-proportional phase incrementation (TPPI), ${ }^{4,49,50}$ hypercomplex data acquisition (also known as the method of States et al. ${ }^{51}$ ), or whole-echo acquisition..$^{52}$

The TPPI and States methods are equivalent in terms of signal-to-noise per unit time." Since TPPI was not used to acquire any of the data in this thesis, it will not be described here, and the interested reader is referred elsewhere. . $^{411,49,50}$

The hypercomplex method of States et al..$^{51}$ permits quadrature "detection" in the indirect dimension. The technique requires the experimenter to collect two separate twodimensional data sets in which the amplitude modulation in the $t_{1}$ dimension differs by $90^{\circ}$ 
between the sets. In practice, the second data set is obtained by shifting the pulse at the beginning or at the end of the $t_{1}$ period by $90^{\circ} / \mathrm{pl}$ where $\mathrm{p}$ is the order of the coherence. ${ }^{4,53}$ The phase cycle must ensure that both the $+p$ and $-p$ coherences are retained throughout the entire $t_{1}$ period. ${ }^{4}$ The retention of both $e^{i \Omega_{1}(p) t_{1}}$ and $e^{-i \Omega_{1}(p) t_{1}}$ leads to a signal with cosinusoidal amplitude modulation, and the $90^{\circ}$ phase shift gives a data set with sinusoidal amplitude modulation. The two signals can be written as

$$
\begin{aligned}
& s_{\cos }\left(t_{1}, t_{2}\right)=\cos \left(\Omega_{1} t_{1}\right) e^{-t_{1} / T_{2}} e^{-i \Omega_{2} t_{2}} e^{-t_{2} / T_{2}} \\
& s_{\sin }\left(t_{1}, t_{2}\right)=\sin \left(\Omega_{1} t_{1}\right) e^{-t_{1} / T_{2}} e^{-i \Omega_{2} t_{2}} e^{-t_{2} / T_{2}}
\end{aligned}
$$

where the -1 quantum coherence is directly detected in $t_{2}$. Fourier transformation in the $t_{2}$ dimension gives

$$
\begin{aligned}
& \mathrm{s}_{\cos }\left(\mathrm{t}_{1}, \omega_{2}\right)=\cos \left(\Omega_{1} \mathrm{t}_{1}\right) \mathrm{e}^{-\mathrm{t}_{1} / \mathrm{T}_{2}}\left(\mathrm{~A}\left(\omega_{2}\right)+\mathrm{iD}\left(\omega_{2}\right)\right) \\
& \mathrm{s}_{\sin }\left(\mathrm{t}_{1}, \omega_{2}\right)=\sin \left(\Omega_{1} \mathrm{t}_{1}\right) \mathrm{e}^{-\mathrm{t}_{1} / \mathrm{T}_{2}}\left(\mathrm{~A}\left(\omega_{2}\right)+\mathrm{iD}\left(\omega_{2}\right)\right) .
\end{aligned}
$$

Combining the real part of Equation (1.126) with i times the real part of Equation (1.127) gives

$$
s_{\text {tot }}\left(t_{1}, \omega_{2}\right)=e^{i \Omega_{1} t_{1}} e^{-t_{1} / T_{2}} A\left(\omega_{2}\right)
$$

Fourier transformation of the $t_{1}$ dimension gives a signal in the real channel, $\mathrm{A}\left(\omega_{1}\right) \mathrm{A}\left(\omega_{2}\right)$, which is purely absorptive.

Two particular linear combinations of Equations (1.124) and (1.125) are known as "echo" and "anti-echo" signals and have the following functional forms

$$
\begin{aligned}
& s_{\text {echo }}\left(t_{1}, t_{2}\right)=s_{\cos }\left(t_{1}, t_{2}\right)-i s_{\sin }\left(t_{1}, t_{2}\right)=e^{-i \Omega_{1} t_{1}} e^{-t_{1} / T_{2}} e^{-i \Omega_{2} t_{2}} e^{-t_{2} / T_{2}} \\
& s_{\text {anti }}\left(t_{1}, t_{2}\right)=s_{\cos }\left(t_{1}, t_{2}\right)+i s_{\sin }\left(t_{1}, t_{2}\right)=e^{+i \Omega_{1} t_{1}} e^{-t_{1} / T_{2}} e^{-i \Omega_{2} t_{2}} e^{-t_{2} / T_{2}} .
\end{aligned}
$$


These phase-modulated signals can be acquired directly by phase cycling to chose the - $p$ coherence for the "echo" signal and the $+\mathrm{p}$ coherence for the anti-echo signal. However, shorter phase cycles can be used if they are acquired as sine and cosine data sets (assuming that one desires to suppress all the coherences in between $-p$ and $+p$ ). Echo and anti-echo data sets are particularly convenient when the position of the maximum of the FID varies as a function of $t_{1}$ since they facilitate the application of shifted apodization functions. ${ }^{53}$ They also are useful when dealing with experiments involving concepts of time reversal. ${ }^{4}$ Details on how to process such spectra are given elsewhere..$^{53}$

An alternative technique for acquiring pure-phase spectra is known as whole-echo acquisition. $^{52}$ In this method, an echo is generated and acquistion started immediately after the echo-forming pulse. Assuming that the shape of the echo envelope is given by two Lorentzian decays centered about the point $\left(t_{2}-\tau\right)$ as shown in Figure 1.6, we can write the collected signal as

$$
s_{\text {whole }}\left(t_{1}, t_{2}\right)=e^{-i \Omega_{1} t_{1}} e^{-t_{1} / T_{2}} e^{-i \Omega_{2}\left(t_{2}-\tau\right)} e^{-\left|t_{2}-\tau\right| / T_{2}}
$$

Note that the phase cycle is such that during $t_{1}$ the $-p$ coherence is selected and the $+p$ coherence is suppressed and that the -1 quantum coherence is directly detected. Fourier transformation of the $t_{2}$ dimension gives

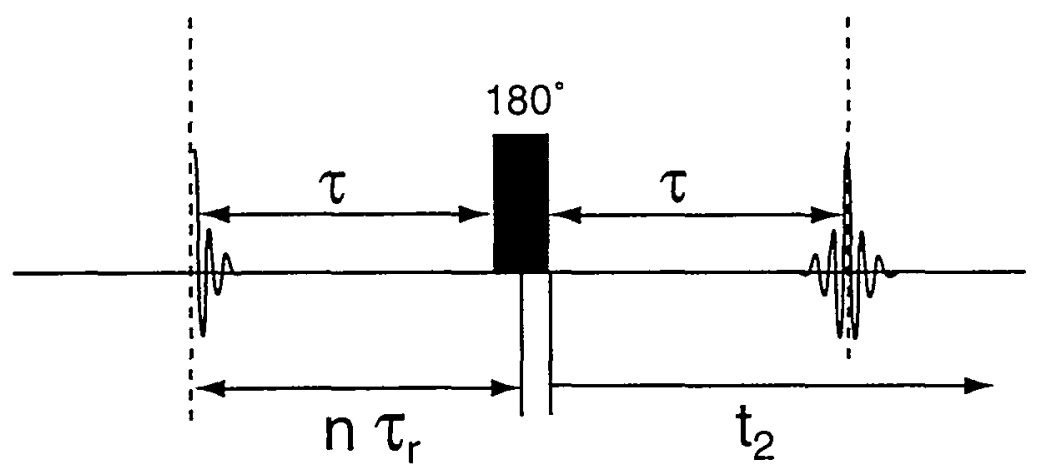

Figure 1.6 - Creation of echoes for whole-echo acquisition. The $180^{\circ}$ echo-forming pulse is placed at a time $\tau$ after the start of the decay. (If MAS is used, the $180^{\circ}$ pulse must be applied after an integral number of rotor periods.) At a time $\tau$ after the $180^{\circ}$, the echo maximum is reached. Acquisition is started immediately after the $180^{\circ}$ pulse so that the entire buildup and decay of the echo is recorded. In the discussion in the text, the finite length of the $180^{\circ}$ pulse is neglected. 


$$
s_{\text {whole }}\left(t_{1}, \omega_{2}\right)=e^{-i \Omega_{1} t_{1}} e^{-t_{1} / T_{2}} \int_{-\infty}^{\infty} e^{-i \Omega_{2}\left(t_{2}-\tau\right)} e^{-\left|t_{2}-\tau\right| / T_{2}} e^{-i \omega_{2} t_{2}} d t_{2}
$$

The key to whole-echo acquisition is that we record the entire buildup and decay of the echo.4,54 This allows us to make the substitution $t^{\prime}=t_{2}-\tau$

$$
s_{\text {whole }}\left(t_{1}, \omega_{2}\right)=e^{-i \Omega_{1} t_{1}} e^{-t_{1} / T_{2}} e^{-i \omega_{2} \tau} \int_{-\infty}^{\infty} e^{-\left|t^{\prime}\right| / T_{2}} e^{-i\left(\omega_{2}+\Omega_{2}\right) t^{\prime}} d t^{\prime}
$$

The time-domain signal is now symmetric about $t^{\prime}=0$. Since it has even parity (see Equation (1.115)), the corresponding frequency-domain signal will have no dispersive components. Explicit integration leads to

$$
s_{\text {whole }}\left(t_{1}, \omega_{2}\right)=e^{-i \Omega_{1} t_{1}} e^{-t_{1} / T_{2}} e^{-i \omega_{2} \tau}\left(\frac{2 T_{2}}{1+\left(\omega_{2}+\Omega_{2}\right)^{2}\left(T_{2}\right)^{2}}\right)
$$

Applying a first-order phase correction of $e^{i \omega_{2} \tau}$ gives

$$
s_{\text {whole }}{ }^{\prime}\left(t_{1}, \omega_{2}\right)=e^{-i \Omega_{1} t_{1}} e^{-t_{1} / T_{2}}\left(\frac{2 T_{2}}{1+\left(\omega_{2}+\Omega_{2}\right)^{2}\left(T_{2}\right)^{2}}\right)
$$

which, as expected, has no dispersive components in the $\omega_{2}$ dimension. Thus, a pure absorption-phase two-dimensional peak can be obtained.

The biggest advantage of whole-echo acquisition is that only one two-dimensional data set needs to be acquired. Thus, the signal-to-noise per unit time is a factor of $\sqrt{2}$ greater than in the hypercomplex experiment. However, whole-echo acquisition will not work for samples with long $T_{2}$ values since the entire echo cannot be acquired in such a case.

It is also possible to combine whole-echo acquisition with States or TPPI to produce spectra with high signal-to-noise ratios. ${ }^{11,53,54}$ 
As the case of whole-echo acquisition shows, an NMR spectroscopist has some flexibility about how to define the origin of the time axis. This becomes particularly important in cases in which the $t_{1}$ dimension is split in $\iota$ multiple segments, ${ }^{4,11,54,55,56}$ (see Chapters 5 and 6). A few such experiments are represented schematically in Figure 1.7. In these experiments (e.g. - MAT, DAS, MQMAS), the $t_{1}$ evolution is split into two or more parts, and an isotropic echo is formed at the end of the last part of the $t_{1}$ period. An anisotropic signal is then recorded during the time $t_{2}$. To obtain spectra which correlate narrow isotropic peaks with broader anisotropic lineshapes, one might naïvely think that one should start acquiring the signal immediately after the $t_{1}$ period ends. However, as can be inferred from examining Figure 1.7a, it is impossible to obtain a pure-phase spectrum with such a sequence. Hypercomplex acquisition won't work because the evolution of the +1 coherence during the last part of the evolution period cannot contribute to the final signal. One potential solution is to shift the $t_{2}$ origin to immediately after the last pulse. Then it is possible to do hypercomplex data acquisition (Figure 1.7b). Note that the definitions of the two time variables have changed, however. The new variables are related to the old variables as follows

$$
\begin{aligned}
& \mathrm{t}_{1}^{\prime}=(1-f) \mathrm{t}_{1} \\
& \mathrm{t}_{2}^{\prime}=\mathrm{ft}_{1}+\mathrm{t}_{2}
\end{aligned}
$$

where $\mathrm{ft}_{1}$ is the last fraction of evolution period. The acquired signal will therefore have the form

$$
\begin{aligned}
& \mathrm{s}_{\text {echo }}\left(\mathrm{t}_{1}{ }^{\prime}, \mathrm{t}_{2}{ }^{\prime}\right)=\mathrm{e}^{-\mathrm{i} \Omega_{1} \mathrm{t}^{\prime}{ }^{\prime}} \mathrm{e}^{-\mathrm{t}_{1}{ }^{\prime} / \mathrm{T}_{2}} \mathrm{e}^{-\mathrm{i} \Omega_{2}\left(\mathrm{t}_{2}{ }^{\prime}-\mathrm{ft}_{1}\right)} \mathrm{e}^{-\mid \mathrm{t}_{2}{ }^{\prime}-\mathrm{ft} \mathrm{t}_{1} / / \mathrm{T}_{2}} \\
& =e^{-i \Omega_{1} t_{1}{ }^{\prime}} e^{-t_{1}{ }^{\prime} / T_{2}} e^{-i \Omega_{2}\left(t_{2}{ }^{\prime}-\frac{f_{1}{ }^{\prime}}{1-f}\right)} e^{-\left|t_{2}{ }^{\prime}-\frac{f_{t}{ }^{\prime}}{1-f}\right| / T_{2}} .
\end{aligned}
$$

Fourier transformation with respect to $t_{2}^{\prime}$ gives

$$
s_{e c h o}\left(t_{1}^{\prime}, \omega_{2}^{\prime}\right)=e^{-i \Omega_{1} t_{1}^{\prime}} e^{-t_{1}^{\prime} / T_{2}} e^{-i \frac{f \omega_{2} t_{1}^{\prime}}{1-f}}\left(\frac{2 T_{2}}{1+\left(\omega_{2}^{\prime}+\Omega_{2}\right)^{2}\left(T_{2}\right)^{2}}\right)
$$


a)
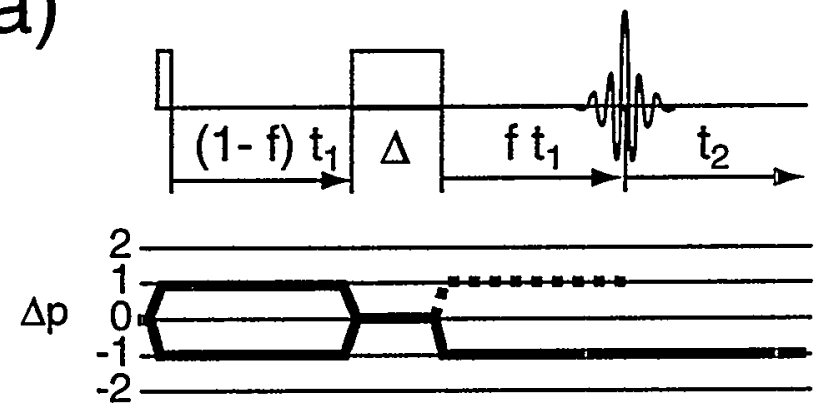

b)
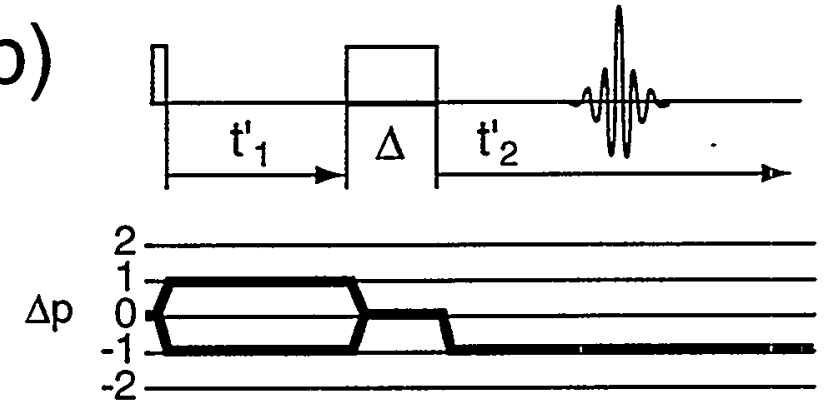

c)
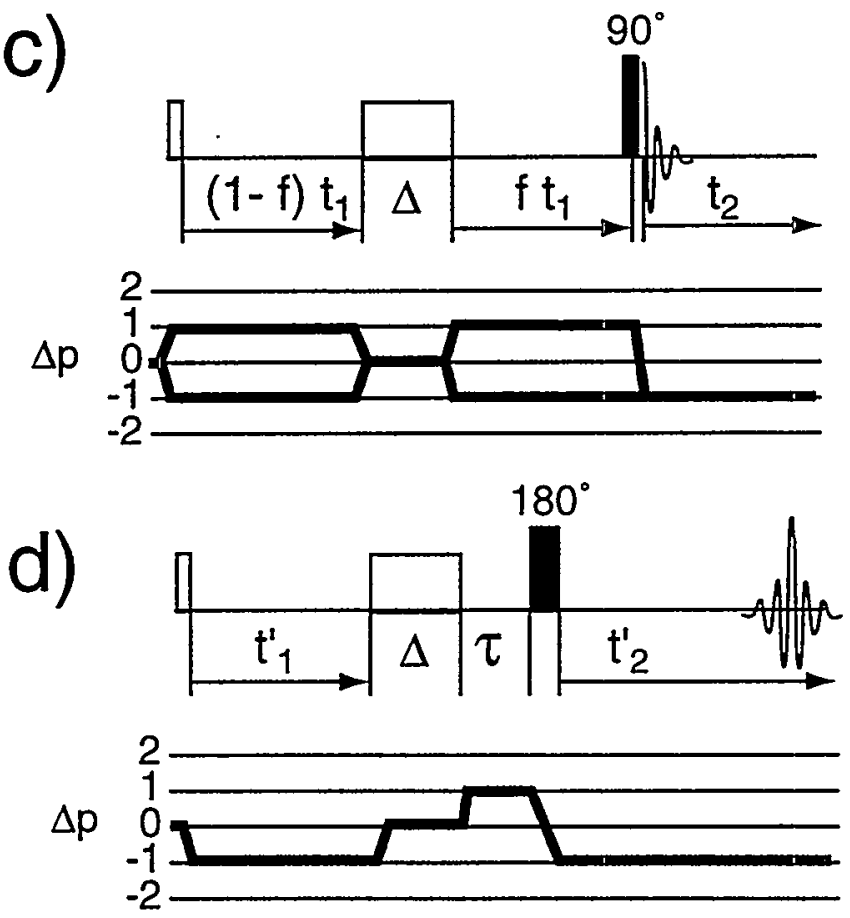

Figure 1.7 - Schematics of pulse sequences with split evolution periods. The white boxes do not represent specific pulses but rather portions of sequences in which the transverse magnetization does not evolve. With the timings shown in (a), pure-phase spectra cannot be obtained since the +1 coherence during the last $t_{1}$ segment (represented by a dashed line) is not recorded. Pure-phase spectra can be obtained with sequences (b), (c), and (d) as described in the text. 
Subsequently transforming with respect to $t_{1}{ }^{\prime}$ and looking at the real part gives

$$
\begin{aligned}
\operatorname{Re}\left[\mathrm{S}_{\mathrm{echo}}\left(\omega_{1}^{\prime}, \omega_{2}^{\prime}\right)\right]= & \left(\frac{\mathrm{T}_{2}}{1+\left(\omega_{1}^{\prime}+\Omega_{1}+\frac{\mathrm{f} \omega_{2}^{\prime}}{1-\mathrm{f}}\right)^{2}\left(\mathrm{~T}_{2}\right)^{2}}\right) \\
& \times\left(\frac{2 \mathrm{~T}_{2}}{1+\left(\omega_{2}^{\prime}+\Omega_{2}\right)^{2}\left(\mathrm{~T}_{2}\right)^{2}}\right)
\end{aligned}
$$

While this is a pure-phase signal, the frequencies in the two dimensions are correlated by the factor $f /(1-f)$, leading to diagonal ridges in the two-dimensional spectrum. For ease of interpretation, however, it would be preferable to have a purely isotropic signal in one dimension and an anisotropic signal in the other dimension. That is, one would like to view this pure-phase spectrum in terms of the frequencies $\left(\omega_{1}, \omega_{2}\right)$ corresponding to the times $\left(t_{1}, t_{2}\right)$ shown in Figure 1.7a. Fortunately, it is possible to have the best of both worlds -- absorptive lineshapes and direct isotropic-anisotropic correlation -- if one shears the spectrum by the angle $e^{4,11,55}$

$$
\Theta_{\text {shear }}=\arctan \left(\frac{f}{1-f}\right)
$$

In practice, the most convenient method for shearing ${ }^{4}$ is to apply a first-order phase correction of $\exp \left[+\mathrm{i}\left(\frac{\mathrm{f} \omega_{2}{ }^{\prime}}{1-\mathrm{f}}\right) \mathrm{t}_{1}{ }^{\prime}\right]$ to Equation (1.139) which leads to

$$
\begin{aligned}
\operatorname{Re}\left[S_{\text {echo }}\left(\omega_{1}{ }^{\prime}, \omega_{2}{ }^{\prime}\right)\right] & =\left(\frac{\mathrm{T}_{2}}{1+\left(\omega_{1}^{\prime}+\Omega_{1}\right)^{2}\left(\mathrm{~T}_{2}\right)^{2}}\right) \\
& \times\left(\frac{2 \mathrm{~T}_{2}}{1+\left(\omega_{2}{ }^{\prime}+\Omega_{2}\right)^{2}\left(\mathrm{~T}_{2}\right)^{2}}\right)
\end{aligned}
$$

Writing Equation (1.143) in terms of the variables $\left(\omega_{1}, \omega_{2}\right)$ gives 


$$
\begin{aligned}
\operatorname{Re}\left[\mathrm{S}_{\mathrm{echo}}\left(\omega_{1}, \omega_{2}\right)\right]= & \left(\frac{\mathrm{T}_{2}}{1+\left(\frac{\omega_{1}}{1-\mathrm{f}}+\Omega_{1}\right)^{2}\left(\mathrm{~T}_{2}\right)^{2}}\right) \\
& \times\left(\frac{2 \mathrm{~T}_{2}}{1+\left(\omega_{2}+\Omega_{2}\right)^{2}\left(\mathrm{~T}_{2}\right)^{2}}\right)
\end{aligned}
$$

where use has been made of Equation (1.136) and the fact that there is reciprocal scaling between the time and frequency domains. ${ }^{4}$ Note that this shearing transformation and concomitant change of variables leads to a spectral width in the $\omega_{1}$ dimension that is a factor of $(1-f)$ of that in the $\omega_{1}^{\prime}$ dimension. This necessitates a rescaling of the $\omega_{1}$ axis in order to obtain the correct isotropic shifts. ${ }^{55}$ It also means that the dwell time for the $t_{1}{ }^{\prime}$ dimension of the experiment in Figure $1.7 \mathrm{~b}$ must be carefully chosen to avoid aliasing. ${ }^{53}$

Pure-phase spectra can also be obtained by slight modifications to the pulse sequence of Figure $1.7 \mathrm{a} / \mathrm{b}$. Insertion of a properly phase-cycled $90^{\circ}$ pulse after the $\mathrm{ft}_{1}$ period can ensure that both +1 and -1 coherences are retained throughout the entire evolution period (Figure 1.7c), allowing direct application of hypercomplex data acquisition. ${ }^{10}$ Alternatively, a $180^{\circ}$ pulse can be inserted after a delay $\tau$ to create a full echo for each $t_{1}$ slice, and the principles of whole-echo acquisition can then be applied (Figure 1.7d). Note that two first-order phase shifts (one associated with whole-echo acquistion and one to shear the spectrum) will be required to process such data. ${ }^{54}$ Examples of many of these methods will be see throughout this thesis. 


\section{Chapter 2: ${ }^{13} \mathrm{C}$ Variable-Temperature CP/MAS Studies of Tetracyclopentadienyl Complexes}

In Chapter 1, the theoretical foundations for solid-state NMR were outlined. In this chapter, an example of how solid-state NMR can be used to probe molecular motion in a series of cyclopentadienyl compounds will be shown. Although this study involves no new techniques, it demonstrates the power of cross polarization, magic-angle spinning, and two-dimensional spectroscopy.

\subsection{Fluxional Motion in Organometallic Compounds}

One of the most important ligands in organometallic chemistry is the cyclopentadienyl ligand, $\mathrm{C}_{5} \mathrm{H}_{5}$ or $\mathrm{Cp}$, which can bind to a metal atom in at least ten different ways. ${ }^{57}$ These different configurations are typically categorized by their hapticity, $\eta$, which indicates the number of carbon atoms that lie close enough to the metal atom to form a bond..$^{58}$ Whether or not all of these carbon atoms actually participate in such a bond is often unclear since the nature of bonding in these compounds is not fully understood. Even the hapticity can be difficult to categorize due to ambiguities in X-ray diffraction data. The existence of monohapto $\left(\eta^{1}\right)$, trihapto $\left(\eta^{3}\right)$, and pentahapto $\left(\eta^{5}\right)$ cyclopentadienyl groups is now generally accepted, but while $\eta^{4}$-cyclopentadienyl groups have been postulated as intermediates in certain cases, their existence remains controversial. ${ }^{57}$ As for the nature of the bonding, it is generally assumed that monohaptocyclopentadienyl groups are bound to the metal atom via a single $\sigma$-bond. In the most common monohapto configuration, the carbon that binds to the metal is $\mathrm{sp}^{3}$ hybridized and the other four carbons are $\mathrm{sp}^{2}$ hybridized. The cyclopentadienyl ring is nearly planar, and contains two double bonds. It is believed that, in most cases, all five carbon atoms in a pentahapto group participate in the bonding through delocalized molecular orbitals: such bonding is called $\pi$ or $\eta$ bonding in older literature.

Describing organometallic compounds in terms of static bonding configurations is not an accurate way to represent many of them, however, since a large number are stereochemically non-rigid. They can undergo rapid intramolecular rearrangements even 
in the solid state. If these rearrangements do not lead to chemically distinct species, the motion is described as fluxional. Fluxional motion was first postulated for the compound $\left(\eta^{5}-\mathrm{C}_{5} \mathrm{H}_{5}\right)\left(\eta^{1}-\mathrm{C}_{5} \mathrm{H}_{5}\right) \mathrm{Fe}(\mathrm{CO})_{2}$ by Piper and Wilkinson in 1956 based on solution-state ${ }^{1} \mathrm{H}$ NMR spectra. ${ }^{59}$ When Piper and Wilkinson failed to see the multiple peaks they expected from a monohaptocyclopentadienyl group, they proposed that rapid ring rearrangements caused the metal-carbon $\sigma$-bond to shift to each carbon atom in turn. Subsequent studies by other workers confirmed this hypothesis, and the motion was termed "ring whizzing." 60 Since then, a variety of possible cyclopentadienyl group motions have been observed including interconversion of $\eta^{3}$ and $\eta^{5}$ ligands, interconversion of $\eta^{1}$ and $\eta^{5}$ ligands, rotation of the $\eta^{3}-\mathrm{C}_{5} \mathrm{H}_{5}$ groups, and rotation about the metal-carbon $\sigma$-bond. ${ }^{57,60,61}$ However, it is the ring-whizzing process which will primarily concern us here.

The mechanism for ring whizzing is difficult to determine a priori and remains controversial today. Since it involves the motion of a sigma bond in a $\pi$-electron system, it can be referred to as a sigmatropic rearrangement. ${ }^{62}$ Sigmatropic rearrangements are identified using the notation $[i, j]$ where the indices $i$ and $j$ indicate over how many atoms each end of the sigma bond migrates. In the case of a metal-carbon bond moving around a cyclopentadienyl ring, several types of rearrangements could occur. Unfortunately, it is impossible to distinguish between a [1,5] shift and a [1,2] shift in a five-membered ring. Thus, if such a shift were observed in an NMR spectrum, one could not determine whether the mechanism involved completely delocalized molecular orbitals $(a[1,5]$ shift $)$ or whether it were based on some sort of "principle of least motion" (a [1,2] shift). ${ }^{58}$ For convenience, such shifts will be referred to as $[1,2]$ shifts throughout the rest of this chapter, but this does not imply that a particular mechanism is favored. Similarly, the other possible type of shift in a five-membered ring will be referred to as a $[1,3]$ shift.

Predicting whether $[1,2]$ shifts, $[1,3]$ shifts, or both are to be expected is nontrivial. Although the molecular orbitals for cyclopentadienyl groups have been determined ${ }^{63,64,65}$, it is difficult to apply the Woodward-Hoffmann rules ${ }^{62}$ to the rearrangements. This is because the transition metal atom has many orbitals of various symmetries which could potentially participate in such a process, especially when the valence shell is not filled. ${ }^{60,66,67}$ Furthermore, the process may be dissociative rather than concerted since metal-carbon bonds are relatively weak. ${ }^{66,67}$ For dissociative processes, a 
Woodward-Hoffmann treatment would not be valid. In the case of cyclopentadienyl groups, Woodward-Hoffmann-based calculations suggest that [1,5] shifts (which are equivalent to $[1,2]$ shifts in a five-membered ring) would be favored. ${ }^{68}$ To date, most studies of the fluxional motion of monohaptocyclopentadienyl compounds have also concluded that $[1,2]$ shifts are the predominant rearrangement mechanism. However, the conclusions that were reached often depended on the assumptions that were used to assign the peaks in an NMR spectrum, and there has not always been a consensus. ${ }^{60.69}$ Moreover, in $\eta^{1}-\mathrm{C}_{7} \mathrm{H}_{7}$ rings, evidence for [1,2], [1,3], and [1,5] shifts has been found despite the fact that only $[1,5]$ shifts are predicted by the Woodward-Hoffman rules. ${ }^{70,66,67}$ "Forbidden" $[1,3]$ shifts have also been observed in five-membered rings in an indenyl ligand bound to $\mathrm{Hg},{ }^{71}$ and a few compounds that were predicted to be static were, in fact, found to be fluxional. ${ }^{66}$ Clearly orbital symmetry considerations are not always the only factors that control fluxional motion.

NMR has proven to be a useful tool for studying several types of Huxional motion. Few other techniques are able to detect rearrangements which do not change the structure of a compound. ${ }^{72}$ Although fluxional motion has been extensively studied in the solution state, the rates can sometimes be too rapid for solution-state NMR to aid in unraveling the mechanism. ${ }^{60}$ Solid-state studies are of paramount importance in understanding such cases although, due to steric considerations, some mechanisms that occur in solution may not necessarily occur in the solid state. ${ }^{73,74}$ The first solid-state NMR studies of fluxional motion used ${ }^{1} \mathrm{H}$ wideline NMR to measure second moments and linewidths as a function of temperature. ${ }^{72.75}$ A series of complexes containing monohaptocyclopentadienyl rings were examined using this method, and the experimental parameters were compared to theoretical models. ${ }^{76}$ Temperature-dependent measurements of relaxation times were used to determine the activation energy for the rearrangment. ${ }^{77}$ However, detailed mechanistic information could not be obtained. Several years later, analysis of ${ }^{13} \mathrm{C}$ NMR static powder line-shapes revealed that jumps through angles of $72^{\circ}$ were favored over jumps of $144^{\circ}$ in $\mathrm{Fe}\left(\eta^{5}-\mathrm{C}_{5}\left(\mathrm{CH}_{3}\right)_{5}\right)_{2}{ }^{78}$ Monitoring ${ }^{13} \mathrm{C}$ CP/MAS lineshapes as a function of temperature also permitted fluxional motion to be studied. ${ }^{74,79,80,81,82}$ Recently, two- 
dimensional exchange NMR has been applied to study the rotation of pentahaptocyclopentadienyl rings in the solid state ${ }^{61,83}$ as well as fluxional motion involving other moieites. ${ }^{84,85}$

In this chapter, the results of a solid-state NMR study of several related organometallic compounds will be presented. All of these compounds have four cyclopentadienyi groups bound to a metal atom in an approximately tetrahedral configuration, and all of the metal atoms have an oxidation state of $\mathrm{V}$. The compound $\mathrm{TiCp}_{4}$ has previously been studied in the solid-state; 82 the compounds that we studied involve metal atoms that usually have a chemistry closely related to that of titanium. Zirconium and hafnium are in the same column in the periodic table as titanium. They are slightly larger and therefore prone to form compounds with higher coordination numbers but are otherwise quite similar in behavior. While tin is not a transition metal element, its chemistry is often also similar to that of titanium since it is the same size, ${ }^{70}$ and extremely rapid fluxional motion in tin compounds has previously been observed. ${ }^{60}$ As will be shown in Section 2.2, the chemistry of $\mathrm{SnCp}_{4}, \mathrm{HfCp}_{4}$, and $\mathrm{ZrCp}_{4}$ is not the same, however.

\subsection{One-Dimensional ${ }^{13} \mathrm{C}$ Variable-Temperature CP/MAS Experiments}

Figure 2.1 depicts the structures of $\mathrm{Sn}\left(\eta^{1}-\mathrm{C}_{5} \mathrm{H}_{5}\right)_{4}, \mathrm{Hf}\left(\eta^{5}-\mathrm{C}_{5} \mathrm{H}_{5}\right)_{2}\left(\eta^{1}-\mathrm{C}_{5} \mathrm{H}_{5}\right)_{2}$, and $\mathrm{Zr}\left(\eta^{5}-\mathrm{C}_{5} \mathrm{H}_{5}\right)_{3}\left(\eta^{1}-\mathrm{C}_{5} \mathrm{H}_{5}\right)$-- which will hereafter be referred to as $\mathrm{SnCp}_{4}, \mathrm{HfCp}_{4}$, and $\mathrm{ZrCp}_{4}$, respectively. The numbers of sigma $\left(\eta^{1}\right)$ and eta $\left(\eta^{5}\right)$ bonded cyclopentadienyl rings in each compound were determined by $\mathrm{X}$-ray diffraction..$^{86,87,88}$

The compounds were synthesized by Drs. Leonidas Phillips, Frances Separovic, Murray S. Davies, and Manuel J. Aroney at the University of Sydney, Sydney, Australia by reacting a metal-halide precursor with sodium cyclopentadienide using methods from the literature. ${ }^{88,89}$ Since the materials were highly hygroscopic, they were shipped in sealed ampules and transferred to $7.5 \mathrm{~mm}$ zirconia pencil rotors in a glove box with a $\mathrm{N}_{2}$ atmosphere. Experiments were typically performed on a given compound for up to a day at a time, and the rotors were stored in a desiccator for several weeks between 
experiments. Only the $\mathrm{SnCp}_{4} \mathrm{NMR}$ spectra changed over time, suggesting that this was the only one of the compounds to undergo significant degradation during the course of the experiments.

All spectra were recorded on a home-built spectrometer ${ }^{90}$ interfaced to a Tecmag pulse programmer and data acquisition system. The ${ }^{1} \mathrm{H}$ Larmor frequency was 301.2 $\mathrm{MHz}$, and the ${ }^{13} \mathrm{C}$ Larmor frequency was $75.739 \mathrm{MHz}$. The probe was home-built but incorporated a $7.5 \mathrm{~mm}$ Chemagnetics MAS spinning module and used a Doty ${ }^{91}$ doubleresonance circuit design. The pulse sequence used for these experiments is shown in Figure 2.2 and is a standard Hartmann-Hahn cross polarization sequence with $\mathrm{CW}$ decoupling on the protons during the acquisition period. Typical proton decoupling field strengths were $35-50 \mathrm{kHz}$.

a)

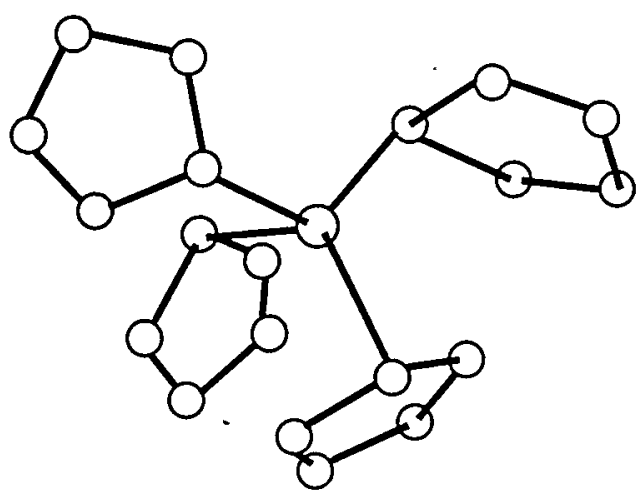

b)

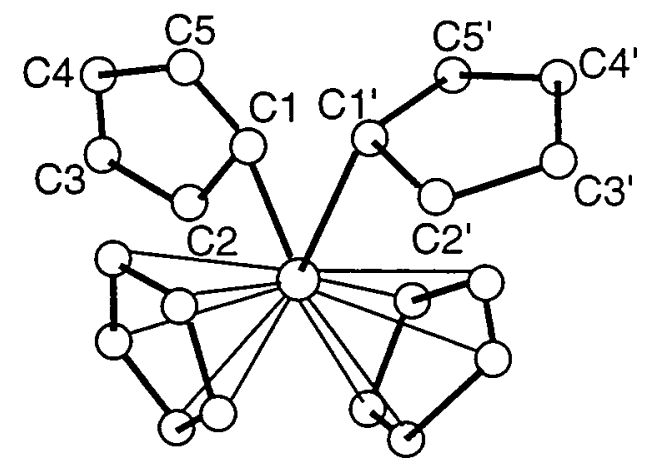

c)

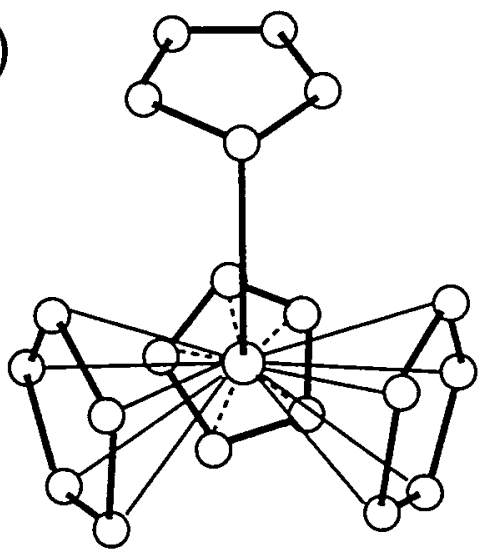

Figure 2.1 - Structures of (a) $\mathrm{Sn}\left(\eta^{1}-\mathrm{C}_{5} \mathrm{H}_{5}\right)_{4}$, (b) $\mathrm{Hf}\left(\eta^{5}-\mathrm{C}_{5} \mathrm{H}_{5}\right)_{2}\left(\eta^{1}-\mathrm{C}_{5} \mathrm{H}_{5}\right)_{2}$, and (c) $\mathrm{Zr}\left(\eta^{5}-\right.$ $\left.\mathrm{C}_{5} \mathrm{H}_{5} 5\right)_{3}\left(\eta^{1}-\mathrm{C}_{5} \mathrm{H}_{5}\right)$. 
The magic angle was set by maximizing the number of rotational echoes in the time-domain ${ }^{79} \mathrm{Br}$ spectrum of $\mathrm{KBr}$. The cross-polarization match condition was set on adamantane and fine-tuned on the actual samples. Typically, the second-sideband match condition $^{92}$ was used which, for a spinning speed of $\sim 4 \mathrm{kHz}$, gave a ${ }^{1} \mathrm{H} 90^{\circ}$ pulse length of $7 \mu$ s and $\mathrm{a}^{13} \mathrm{C} 90^{\circ}$ pulse length of $9 \mu \mathrm{s}$. The cross-polarization contact time was $1 \mathrm{~ms}$.

To obtain temperatures in the range $133-333 \mathrm{~K}$, the variable-temperature apparatus depicted in Figure 2.3 was used. In this set-up, gaseous $\mathrm{N}_{2}$ was cooled by being passed through a coil immersed in a liquid nitrogen bath. It was transported to the probe through a heater and an insulated stack placed in the bore of the magnet. A thermocouple located in the stack was interfaced to a temperature-controller which regulated the temperature of the $\mathrm{N}_{2}$ gas to within $\pm 5 \mathrm{~K}$. The design of the MAS spinning module was such that the rotor was supported by air bearings at either end, and the rf-coil was free-standing. This permitted the temperature-controlled gas to access a large portion of the rotor through holes at the top of the stator. $\mathrm{N}_{2}$ gas was also used for both the bearing and the drive air lines to minimize water condensation in the probe.

During the course of the experiments the temperature was decreased in steps of 10 $\mathrm{K}$ and the sample was allowed to equilibrate for at least twenty minutes at each temperature before spectra were recorded.

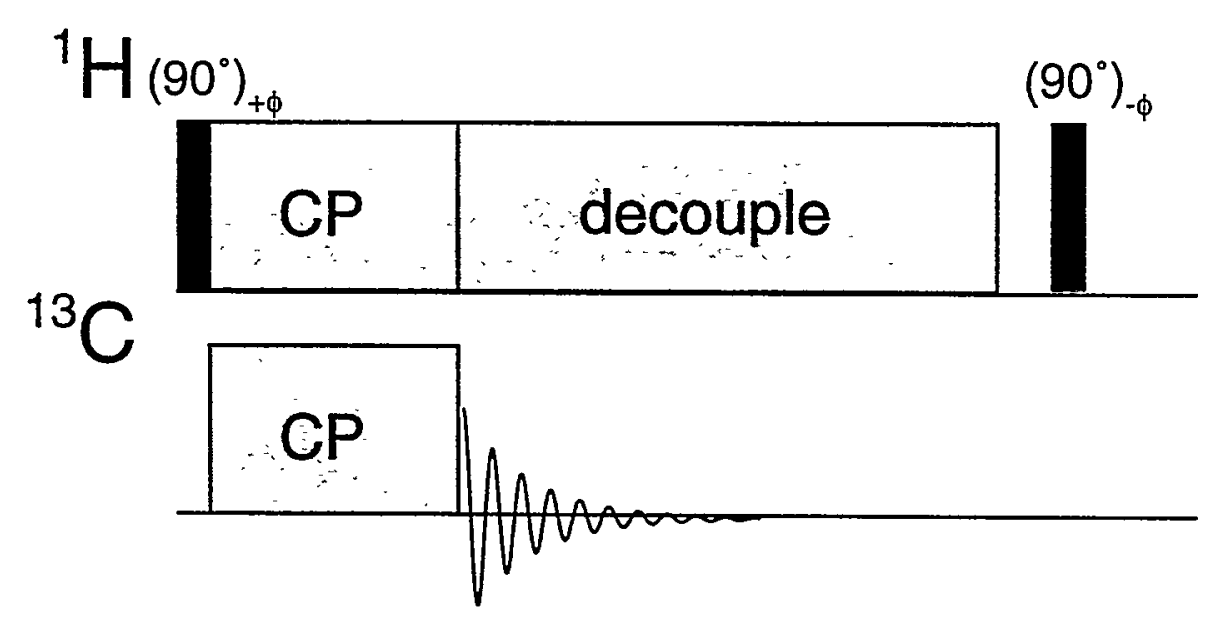

Figure 2.2 - Cross polarization pulse sequence for 1D VT-MAS experiments. CYCLOPS phase cycling ${ }^{46}$ and spin-temperature alternation ${ }^{47}$ were used. A flipback pulse at the end of the sequence returned any remaining spin-locked proton magnetization back to the zaxis. 
To calibrate the controller, an independent series of experiments were performed on the compound samarium acetate $\left(\mathrm{SmAc}_{3}\right)$ tetrahydrate using the same pulse sequence and spinning speed. Since the ${ }^{13} \mathrm{C}$ isotropic chemical shift of the chelating carbonyl group in $\mathrm{SmAc}_{3}$ is temperature-sensitive ${ }^{93,94}$ with a temperature dependence given by

$$
\delta_{\text {iso }}[\mathrm{ppm}]=-\frac{4867}{\mathrm{~T}}+209 \text {, }
$$

such experiments were used to establish a correlation between the temperature reading from the thermocouple and the actual temperature of the sample.

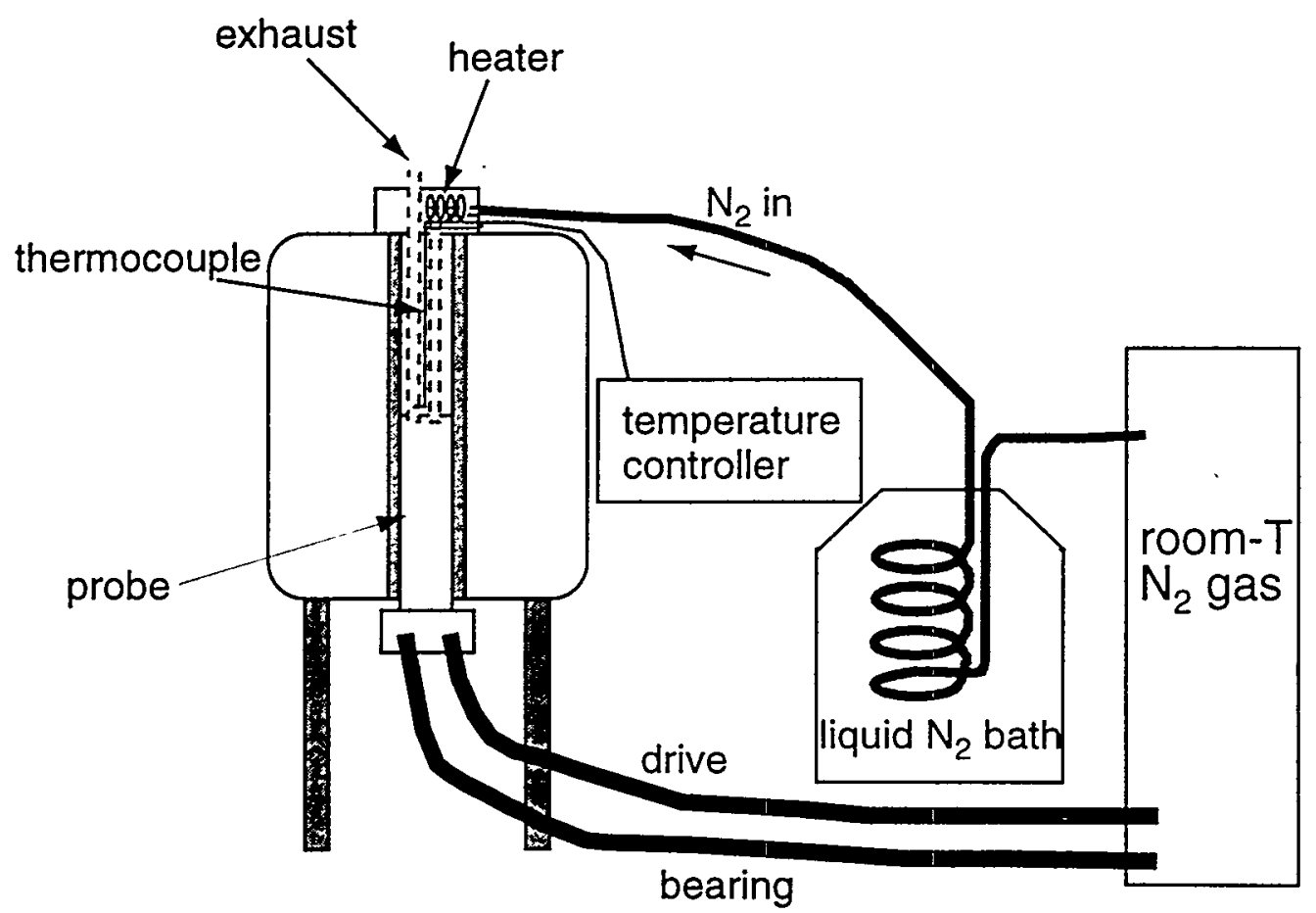

Figure 2.3 - Schematic of cooling apparatus used for variable-temperature MAS experiments. Bearing and drive air for the MAS probe were supplied from a high pressure (300 psi) $\mathrm{N}_{2}$ dewar. Sample cooling was achieved by blowing $\mathrm{N}_{2}$ gas through a copper coil located in a liquid- $\mathrm{N}_{2}$ bath. The heating coil and thermocouple in the VT stack were interfaced to a temperature controller which provided a feedback loop to regulate the temperature. 


\subsection{1 $\mathrm{SnCp}_{4}$ Spectra}

Fluxional motion in tin compounds tends to be very rapid, which means extremely low temperatures are needed to characterize it. ${ }^{60}$ Previous solution-state ${ }^{1} \mathrm{H}$ NMR studies of $\mathrm{SnCp}_{4}$ in various solvents showed only a single resonance even at temperatures as low as $138 \mathrm{~K}$, which prevented mechanistic information from being determined. ${ }^{95}$

$\mathrm{X}$-ray diffraction studies of $\mathrm{SnCp}_{4}$ showed that all cyclopentadienyl groups were monohapto and were bound to the tin atom in a distorted tetrahedral configuration through elongated $\sigma$-bonds (see Figure 2.1a). ${ }^{86}$ The crystal showed relatively low (monoclinic) symmetry with large variations in the angles between the planes of the cyclopentadienyl rings.

Figure 2.4 shows a series of one-dimensional MAS spectra of $\mathrm{SnCp}_{4}$ recorded at different temperatures. The poor signal-to-noise of these spectra is due to the fact that the $\mathrm{T}_{1}$ of the protons in this sample is rather long at low temperatures, requiring recycle delays of $40 \mathrm{~s}$.

At room temperature, only a single peak at $114.0 \mathrm{ppm}$ was observed in the spectrum of $\mathrm{SnCp}_{4}$. As the sample was cooled to $233 \mathrm{~K}$, two additional broad peaks began to appear at approximately $130 \mathrm{ppm}$ and $50 \mathrm{ppm}$. As the sample was cooled still further, the $114.0 \mathrm{ppm}$ peak diminished in size, while the broad peaks continued to increase in intensity. Finally, at $153 \mathrm{~K}$, the $114.0 \mathrm{ppm}$ peak had disappeared entirely, leaving only the two broader peaks.

A definitive interpretation of these spectra is difficult due to their poor sensitivity and resolution. Some of the sidebands overlap with some of the resonances, and the recycle delay may not have been long enough to ensure that the spectra are quantitative. Furthermore, $\mathrm{SnCp}_{4}$ was found to be extremely sensitive to moisture, and a change in the appearance of the room-temperature spectra over several experimental sessions (data not shown) indicated that the sample easily degraded. Although the spectra shown in Figure 2.4 were acquired on a freshly-packed sample, the integrity of the sample cannot be guaranteed, and therefore all interpretations of the spectra are provisional. Nonetheless, room temperature spectra acquired before and after the low-temperature experiments 

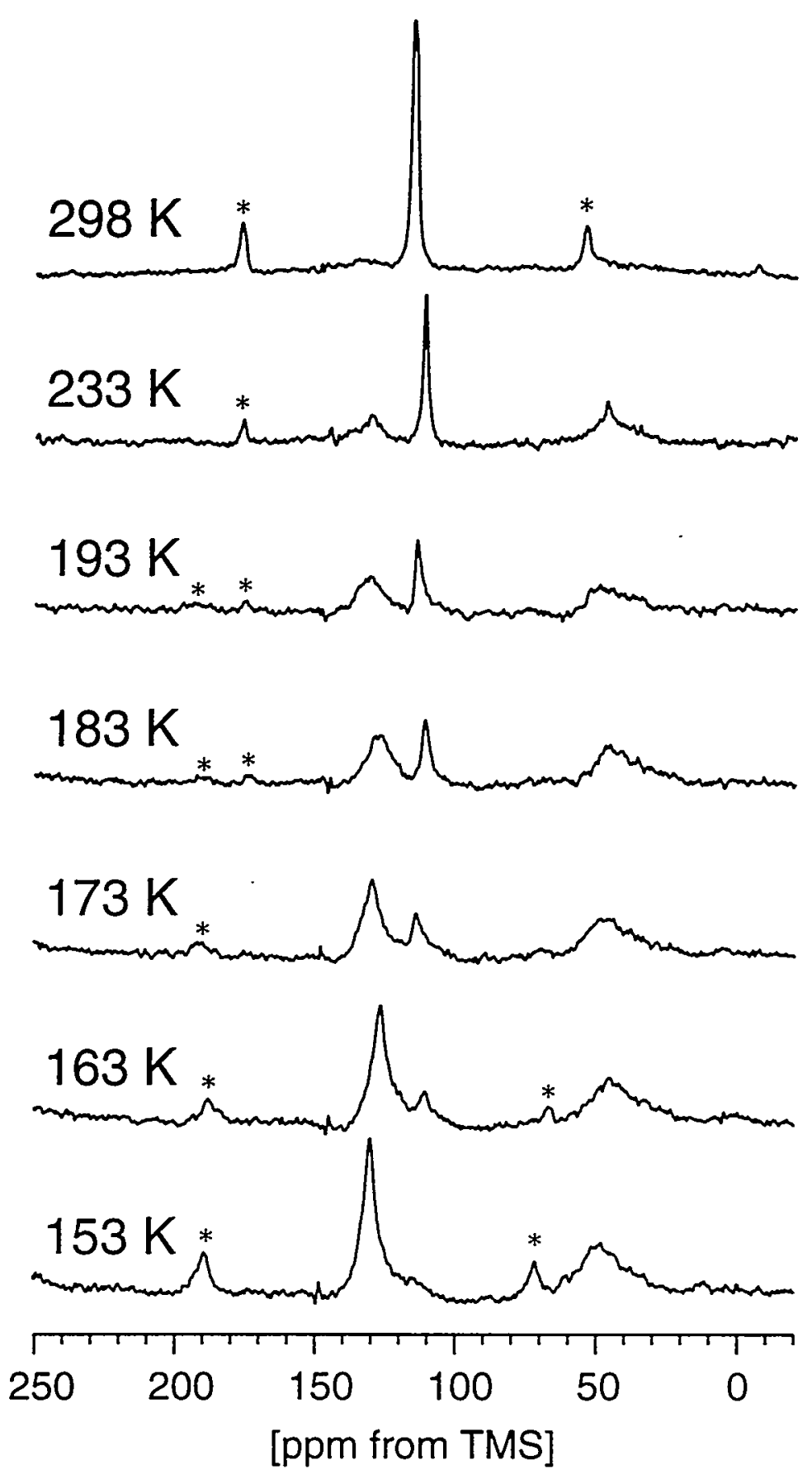

Figure 2.4 - ${ }^{13} \mathrm{C}$ CPMAS spectra of $\mathrm{Sn}\left(\eta^{1}-\mathrm{C}_{5} \mathrm{H}_{5}\right)_{4}$ at different temperatures. The spectra are unscaled, and asterisks are used to denote spinning sidebands in cases where they do not overlap significantly with peaks. Chemical shifts are in ppm from TMS. For all of the spectra except for the room-temperature one, 128 scans were recorded with a recycle delay of $40 \mathrm{~s}$. (The room temperature spectrum resulted from the acquisition of 64 scans with a $10 \mathrm{~s}$ recycle delay.) 
appeared identical, which supports the hypothesis that the broad peaks in Figure 2.4 represent motional processes rather than degradation of the complex due to absorption of water.

One possible interpretation of the spectra in Figure 2.4 is that the individual monohaptocyclopentadienyl rings in $\mathrm{SnCp}_{4}$ have different activation energy barriers for ring rearrangement. At $298 \mathrm{~K}$, the temperature was sufficiently high that all ring rearrangements were rapid, and a single, motionally averaged peak was seen. As the temperature was lowered, the rings with the highest activation energy barriers were now in the slow motion regime. In this limit, one would expect to see distinct and broad resonances for each of the five types of carbons in these rings (see Section 2.2.2). These resonances would then be expected to narrow as the temperature was lowered still further. Indeed, the resonances at approximately 130 and $50 \mathrm{ppm}$ were quite broad at $193 \mathrm{~K}$, and the $130 \mathrm{ppm}$ signal narrowed appreciably at lower temperatures. Although only two peaks (rather than five) were observed, the sum of the intensity of the peak at $130 \mathrm{ppm}$ and its sidebands is clearly greater than the intensity of the peak at $50 \mathrm{ppm}$ in the spectrum at 153 $\mathrm{K}$, indicating that more carbons contribute to the signal at $130 \mathrm{ppm}$. Further quantification was not attempted due to the limited resolution of this data. However, the empirical tendency ${ }^{96}$ for $\mathrm{sp}^{3}$-hybridized carbons to be more shielded than $\mathrm{sp}^{2}$-hybridized carbons suggests that the peak at $50 \mathrm{ppm}$ be assigned to the sigma-bonded carbons in the cyclopentadienyl rings and that the peak at $130 \mathrm{ppm}$ is due to the four remaining carbons. Further support for this assignment comes from the fact that $114 \mathrm{ppm}$ is the weighted average of four resonances at $\sim 130 \mathrm{ppm}$ and one resonance at $\sim 50 \mathrm{ppm}$.

As the temperature was lowered from $233 \mathrm{~K}$ to $153 \mathrm{~K}$, cyclopentadienyl rings with lower activational energy barriers entered the slow-motion regime. Thus, the intensity of the peak at $114.0 \mathrm{ppm}$ monotonically decreased while the intensity of the broad peaks increased. Finally, at $153 \mathrm{~K}$, the peak at $114.0 \mathrm{ppm}$ disappeared almost entirely, indicating that at this temperature few of the ring rearrangements were occurring rapidly on the NMR timescale.

Unfortunately, it was not possible with our experimental apparatus to be sure that the motion was frozen out entirely. However, it is likely that the broad peaks at $153 \mathrm{~K}$ were due to a dispersion of the isotropic chemical shifts for the different sites rather than 
motional effects. $\mathrm{X}$-ray crystallographic results show that steric distortions are present in crystalline $\mathrm{SnCp}_{4} ;{ }^{86}$ such distortions might be responsible both for variations in isotropic shifts and for the range of activation energy barriers.

Because of the long spin-lattice relaxation times and poor site resolution, twodimensional exchange experiments were not performed on this compound. Consequently, the ring rearrangement mechanism could not be determined.

A Herzfeld-Berger analysis ${ }^{40}$ was performed on the room-temperature spectrum to determine the motionally averaged values of the carbon chemical-shielding tensor. The following values were obtained: $\sigma_{11}=165 \pm 8 \mathrm{ppm}, \sigma_{22}=146 \pm 15 \mathrm{ppm}, \sigma_{33}=23 \pm 10$ ppm, $\eta^{C S}=0.2 \pm 0.3 \mathrm{ppm}, \delta^{C S}=-89 \pm 10 \mathrm{ppm}$. A previously reported analysis of the room temperature ${ }^{13} \mathrm{C}$ powder lineshape found values of $\sigma_{11}=180 \mathrm{ppm}, \sigma_{22}=143 \mathrm{ppm}$, and $\sigma_{33}=31 \mathrm{ppm} .{ }^{95}$ The authors of this study did not report error bars for this fit, but the signal-to-noise ratio was such that the error bars should be sizeable. Our results, therefore, are not inconsistent with theirs.

\subsection{2 $\mathrm{HfCp}_{4}$ Spectra}

The compound $\mathrm{HfCp}_{4}$ has two monohaptocyclopentadienyl rings and two pentahaptocylopentadienyl rings (see Figure 2.1b), which make it similar in structure to $\mathrm{TiCp}_{4}$. X-ray diffraction studies of $\mathrm{HfCp}_{4}$ show that, unlike in the case of the $\mathrm{SnCp}_{4}$ compound, considerable molecular symmetry is present. ${ }^{87,97}$ The two eta rings are magnetically equivalent and the two sigma rings are related by a two-fold axis of symmetry. In fact, as will be shown below, the corresponding primed and unprimed monohaptocyclopentadienyl carbons in Figure $2.1 \mathrm{~b}$ resonate at the same frequency. In the rest of this chapter, the unprimed labels $(\mathrm{C} 1, \mathrm{C} 2, \mathrm{C} 3$, etc.) will be used to refer to both carbons. The double bonds are between $\mathrm{C} 2$ and $\mathrm{C} 3$ and between $\mathrm{C} 4$ and $\mathrm{C} 5$.

Solution-state ${ }^{1} \mathrm{H}$ NMR studies of both $\mathrm{HfCp}_{4}$ and $\mathrm{TiCp}_{4}$ have been performed previously. ${ }^{98}$ While ring interchange was observed for $\mathrm{TiCp}_{4}$, no change was seen in the ${ }^{1} \mathrm{H}$ spectrum of $\mathrm{HfCp}_{4}$ at temperatures as low as $123 \mathrm{~K}$. A solid state study of $\mathrm{HfCp}_{4}$ was, therefore, desirable. 
Figure 2.5 shows the variable-temperature, one-dimensional ${ }^{13} \mathrm{C}$ CP/MAS spectra of $\mathrm{HfCp}_{4}$ for the same temperature range as in Section 2.2.1. The room temperature spectrum shows a single peak (at $113.0 \mathrm{ppm}$ ) and its spinning sidebands. Herzfeld-Berger analysis ${ }^{40,99}$ of this spectrum reveals that the chemical-shielding tensor associated with this site is axially symmetric, which is what is expected for a rapidly moving $\eta^{5}-\mathrm{C}_{5} \mathrm{H}_{5}$ group. ${ }^{82}$ Thus, the peak at $113.0 \mathrm{ppm}$ was assigned to the ten carbon atoms of the two $\eta^{5}-\mathrm{C}_{5} \mathrm{H}_{5}$ rings. This peak retained its intensity and its symmetry throughout the range of temperatures studied, indicating that the activation energy barrier for eta ring reorientation is very low. This motion may also provide an efficient relaxation mechanism for the protons at low temperature: the ${ }^{1} \mathrm{H} \mathrm{T}_{1}$ of $\mathrm{HfCp}_{4}$ is approximately $1.7 \mathrm{~s}$ at room temperature, significantly shorter than the $\mathrm{T}_{1}$ of $\mathrm{SnCp}_{4}$.

Absent from the spectrum at $298 \mathrm{~K}$ was direct evidence of the carbons from the sigma rings. These carbons may have contributed to the broad baseline in this spectrum. Alternatively, the motion of these groups could have occurred at a similar rate to the decoupling frequency, leading to destructive interference and loss of signal. ${ }^{100}$ As the temperature was lowered, peaks due to the monohaptocyclopentadienyl rings became evident: first as broad humps at $213 \mathrm{~K}$ and later as well-defined resonances at 90.0, 126.5, 127.9 , and $130.7 \mathrm{ppm}$. These resonances correspond to four of the five types of carbons on the $\eta^{1}-\mathrm{C}_{5} \mathrm{H}_{5}$ ring. The fifth resonance was assumed to lie under the intense $\eta^{5}-\mathrm{C}_{5} \mathrm{H}_{5}$ resonance because this large peak had six times the intensity of the smaller peaks; this assignment was subsequently confirmed by two-dimensional exchange experiments (see Section 2.3). As in the case of $\mathrm{SnCp}_{4}$, the most-shielded monohapto carbon resonance can be assigned to the carbon sigma-bonded to the metal group since $\mathrm{sp}^{3}$-hybridized carbons tend to be more shielded than $\mathrm{sp}^{2}$-hybridized carbons. ${ }^{96}$ The issues involved in assignment of the remaining resonances will be discussed in Sections 2.3 and 2.4.

Table 2.1 shows the principal values of the ${ }^{13} \mathrm{C}$ chemical shielding tensor for each of the sites in $\mathrm{HfCp}_{4}$. These were extracted from a spectrum of $\mathrm{HfCp}_{4}$ recorded at $133 \mathrm{~K}$ (data not shown) in which the motion of the sigma rings was assumed to be frozen out. (No cross peaks were seen in two-dimensional exchange spectra at this temperature for mixing times as long as $500 \mathrm{~ms}$.) Herzfeld-Berger spinning sideband intensity analysis ${ }^{40}$ was used to determine the principal values and a modified version of the Speedyfit ${ }^{99}$ 


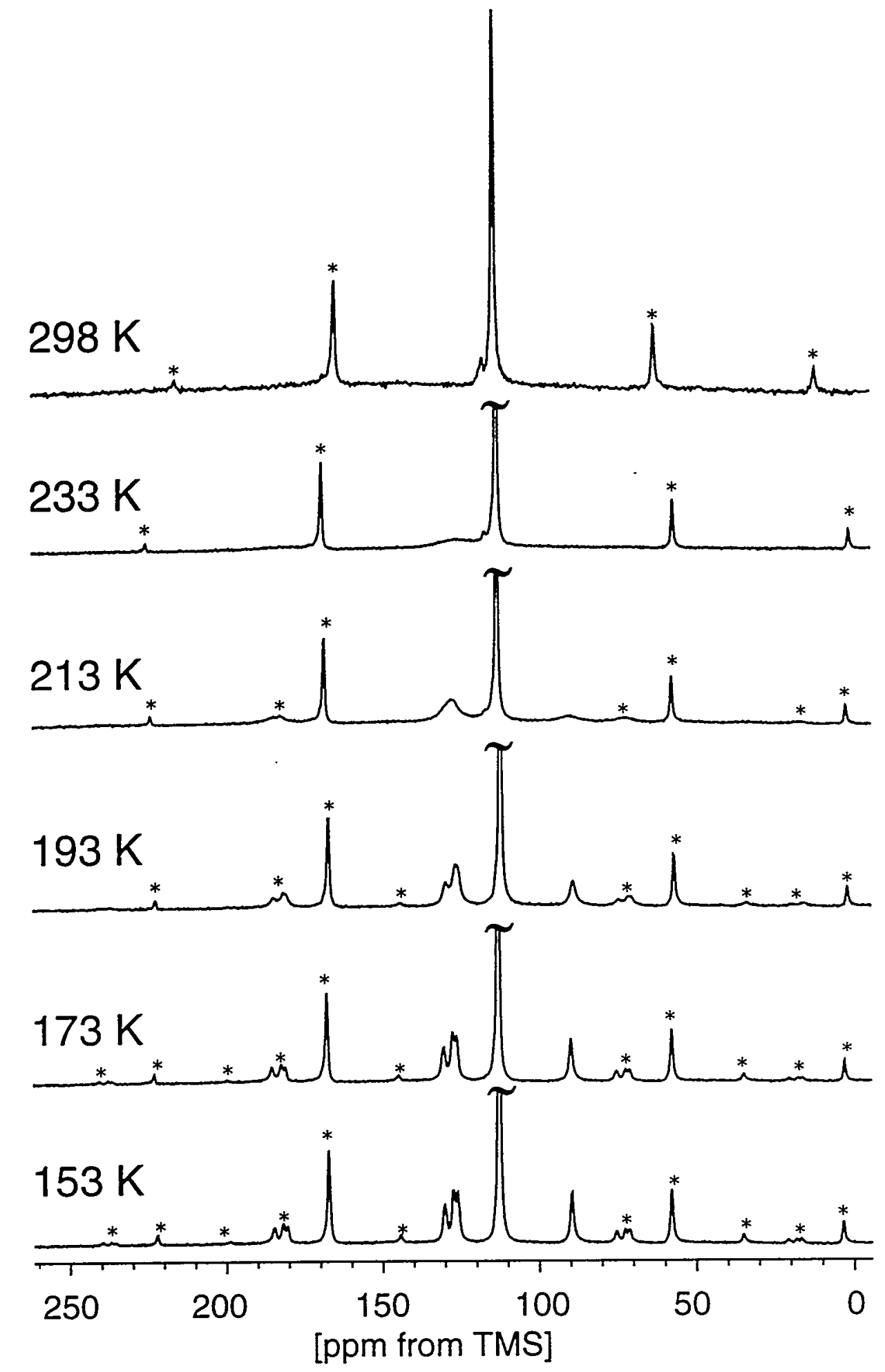

Figure $2.5-{ }^{13} \mathrm{C}$ CP/MAS spectra of $\mathrm{Hf}\left(\eta^{5}-\mathrm{C}_{5} \mathrm{H}_{5}\right)_{2}\left(\eta^{1}-\mathrm{C}_{5} \mathrm{H}_{5}\right)_{2}$ at different temperatures. The spectra are scaled to the height of the tallest peak, but that peak is truncated in these plots. Asterisks are used to denote spinning sidebands. Chemical shifts are in ppm from TMS. For each of these spectra, 512 scans were recorded with a recycle delay of $3 \mathrm{~s}$. 
program was used to deconvolve the partially overlapping lines by iteratively fitting a simulated spectrum to the experimental spectrum. Previous measurements of the ${ }^{13} \mathrm{C}$ CSA in this compound were performed on a static sample and, therefore, were an average over all sites. ${ }^{95}$

\begin{tabular}{|c|c|c|c|c|c|}
\hline $\begin{array}{c}\text { Isotropic } \\
\text { Shift [ppm] }\end{array}$ & $\sigma_{\mathbf{1 1}}$ [ppm] & $\sigma_{\mathbf{2 2}}$ [ppm] & $\sigma_{\mathbf{3 3}}$ [ppm] & $\eta^{\mathbf{C S}}$ & $\delta^{\mathbf{C S}}$ [ppm] \\
\hline \hline 90.0 & $157 \pm 1$ & $90 \pm 1$ & $23 \pm 1$ & $1.00 \pm 0.04$ & $-67 \pm 1$ \\
\hline $113.0^{\mathrm{a}}$ & $162 \pm 1$ & $162 \pm 1$ & $15 \pm 1$ & $0.00 \pm 0.01$ & $-98 \pm 1$ \\
\hline 126.5 & $197 \pm 3$ & $157 \pm 6$ & $24 \pm 4$ & $0.39 \pm 0.10$ & $-102 \pm 4$ \\
\hline 127.9 & $199 \pm 4$ & $158 \pm 6$ & $26 \pm 4$ & $0.41 \pm 0.11$ & $-102 \pm 4$ \\
\hline 130.7 & $220 \pm 2$ & $132 \pm 3$ & $39 \pm 2$ & $0.97 \pm 0.06$ & $-91 \pm 2$ \\
\hline
\end{tabular}

Table 2.1 - Principal values of the ${ }^{13} \mathrm{C}$ chemical-shielding tensor for individual sites in $\mathrm{HfCp}_{4}$.

a. This peak is a superposition of two peaks: an intense resonance from the pentahapto group and a smaller resonance (with one-fifth the intensity) from one of the carbons in the monohapto group. Thus, the corresponding principal values reflect a weighted average of the two.

The intensity of the peak at $113.0 \mathrm{ppm}$ is primarily due to the pentahapto carbons although it also contains contributions from one of the monohapto carbons. Still, the relative sideband intensities are dominated by the pentahapto carbons, and the chemicalshielding tensor appears axially symmetric.

The principal values of the chemical shielding tensor for the other sites are similar to those found in the literature. ${ }^{24,101}$ Based on a compilation of data from fifty-three compounds, T. M. Duncan ${ }^{24}$ has determined that a typical olefinic carbon nucleus has an isotropic chemical shift of $131 \mathrm{ppm}$ (with a standard deviation of $10 \mathrm{ppm}$ ) and principal chemical shielding tensor components of $\sigma_{11}=224 \pm 16 \mathrm{ppm}, \sigma_{22}=134 \pm 21 \mathrm{ppm}$, and $\sigma_{33}=37 \pm 15 \mathrm{ppm}$. The $126.5,127.9$, and $130.7 \mathrm{ppm}$ resonances in the spectrum of $\mathrm{HfCp}_{4}$ all have tensor components that fall within two standard deviations of these values, while the $\sigma_{22}$ and especially the $\sigma_{11}$ components of the $90.0 \mathrm{ppm}$ site lie outside this range. This further supports the assignment of the $90.0 \mathrm{ppm}$ resonance to the $\mathrm{C} 1$ carbon. However, empirical correlations of the chemical-shielding tensor components do not permit a definitive assignment of the $\mathrm{C} 2, \mathrm{C} 3, \mathrm{C} 4$, and $\mathrm{C} 5$ carbons. 
Two-dimensional exchange experiments were successfully performed on $\mathrm{HfCp}_{4}$ and will be discussed in Section 2.3.

\subsection{3 $\mathrm{ZrCp}_{4}$ Spectra}

The $\mathrm{X}$-ray structure of $\mathrm{ZrCp}_{4}$ was highly controversial for many years. Although the structure depicted in Figure 2.1c was first proposed as early as $1970,{ }^{102}$ it was immediately challenged by other researchers. ${ }^{103}$ The primary reason for this was that a $\mathrm{Zr}\left(\eta^{5}-\mathrm{C}_{5} \mathrm{H}_{5}\right)_{3}\left(\eta^{1}-\mathrm{C}_{5} \mathrm{H}_{5}\right)$ configuration would imply that the zirconium atom had twenty electrons in its valence shell (four from the zirconium, five from each pentahapto cyclopentadienyl group, and one from the monohapto group). This violates the wellestablished "18-electron rule" which predicts that transition-metal elements strive to achieve a closed-shell configuration. While exceptions to the 18-electron rule (such as $\mathrm{TiCp}_{4}$ and $\mathrm{HfCp}_{4}$ ) are known, they tend to be in the other direction; that is, elements on the left side of the d-block often have fewer than eighteen valence electrons because of steric considerations. ${ }^{58}$ In fact, this is often proposed as a reason for the instability of such compounds. ${ }^{104}$ In 1978, however, a more accurate X-ray structure determination showed that $\mathrm{ZrCp}_{4}$ does indeed have the configuration shown in Figure 2.1c, although the $\mathrm{Zr}-\mathrm{C}$ bond lengths were significantly longer than the typical case. ${ }^{88}$ It may be possible to think of these extended bond lengths as indicative of effectively fewer electrons being donated per Cp group; 8889 this would then allow an 18-electron configuration to be achieved.

Another surprising aspect of this structure was that it differed significantly from $\mathrm{HfCp}_{4}{ }^{87}$ Generally, it has been assumed that zirconium and hafnium have the same organometallic chemistry. ${ }^{89}$ However, the X-ray structures showed that this was clearly not the case (see Figure 2.1). Variable-temperature solution-state ${ }^{1} \mathrm{H}$ NMR studies of $\mathrm{ZrCp}_{4}$ have been performed but showed no change in the spectrum for temperatures as low as $123 \mathrm{~K} .{ }^{98}$

In Figure 2.6 a series of one-dimensional, variable-temperature ${ }^{13} \mathrm{C}$ CP/MAS spectra of $\mathrm{ZrCp}_{4}$ are shown. A strong pentahapto peak at $112.8 \mathrm{ppm}$ was visible at all temperatures studied. Already at room temperature, resonances due to the monohaptocyclopentadienyl groups were evident; therefore, higher temperature 


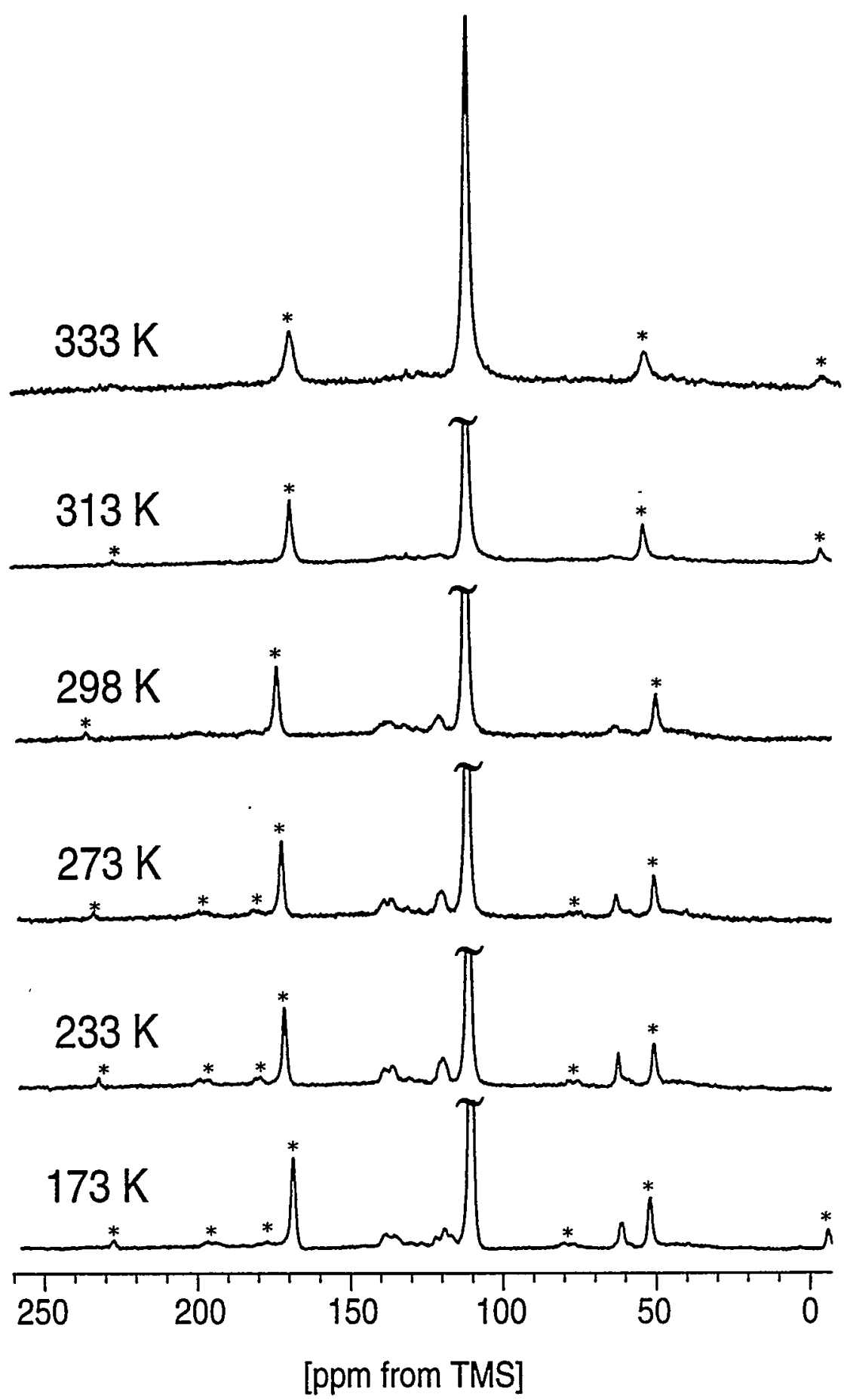

Figure $2.6-{ }^{13} \mathrm{C} \mathrm{CP/MAS} \mathrm{spectra} \mathrm{of} \mathrm{Zr}\left(\eta^{5}-\mathrm{C}_{5} \mathrm{H}_{5}\right)_{3}\left(\eta^{1}-\mathrm{C}_{5} \mathrm{H}_{5}\right)$ at different temperatures. The spectra are scaled to the height of the tallest peak, but that peak is truncated in these plots. Asterisks are used to denote spinning sidebands. Chemical shifts are in ppm from TMS. For each of these spectra, 512 scans were recorded with a recycle delay of $3 \mathrm{~s}$. 
experiments were performed to reach the fast-exchange limit. At $173 \mathrm{~K}$, five distinct resonances from the carbons in the monohaptocyclopentadienyl ring were observed at $63.7,119.1,124.3,138.3$, and $141.0 \mathrm{ppm}$. A sixth resonance at $121.4 \mathrm{ppm}$ was actually a sideband of the $63.7 \mathrm{ppm}$ site; with the achievable spinning speeds of the probe, it was not possible to prevent the sidebands and centerbands from overlapping. Consequently, Herzfeld-Berger fits could not be performed on the monohapto carbons. The principal values of the pentahapto carbon chemical shielding tensor were determined to have the following values: $\sigma_{11}=157 \pm 5 \mathrm{ppm}, \sigma_{22}=156 \pm 9 \mathrm{ppm}, \sigma_{33}=23 \pm 5 \mathrm{ppm}, \eta^{\mathrm{CS}}=0.0 \pm$ $0.1 \mathrm{ppm}, \delta^{\mathrm{CS}}=-89 \pm 5 \mathrm{ppm}$. To within the accuracy of the fitting procedure, the expected axial symmetry was observed. The ${ }^{13} \mathrm{C}$ CSA of this compound has been previously measured by another group, ${ }^{95}$ but their measurements were on a static sample which prevented them from distinguishing between monohapto and pentahapto cyclopentadienyl rings.

Two-dimensional exchange experiments on $\mathrm{ZrCp}_{4}$ were attempted for temperatures in the range of 198-298 $\mathrm{K}$ and for mixing times as long as $500 \mathrm{~ms}$, but no cross peaks were observed. Ring rearrangement in $\mathrm{ZrCp}_{4}$ may occur on a slower timescale, however.

\subsection{Two-dimensional Exchange Spectroscopy}

While the one-dimensional spectra of $\mathrm{HfCp}_{4}$ in Section 2.2.2 indicated that motion of the monohaptocyclopentadienyl rings was occurring, the nature of that motion remained to be determined. A variety of possible dynamical processes such as monohapto ring flips, exchange between monohapto and pentahapto rings, [1,2] sigmatropic rearrangements, and $[1,3]$ sigmatropic rearrangements may have been taking place.

To probe the dynamics in solid $\mathrm{HfCp}_{4}$, several types of experiments could be performed. One possibility is one-dimensional magnetization transfer experiments ${ }^{105,106}$ which can indicate exchange between a given pair of sites. However, in many ways, twodimensional experiments are preferable since they can provide information about many exchanging sites simultaneously. This examination of the dynamics of $\mathrm{HfCp}_{4}$ is believed to be the first instance in which two-dimensional exchange NMR was used to study the fluxional motion of $\eta^{1}-\mathrm{C}_{5} \mathrm{H}_{5}$ groups. 
Figure 2.7 shows the pulse-sequence, coherence-transfer pathway, and phase cycle for the two-dimensional exchange experiment performed on $\mathrm{HfCp}_{4}$. In this sequence, the cross-polarization step creates carbon magnetization which evolves for a time period $t_{1}$. Then this magnetization is flipped along the $\mathrm{z}$-axis" for a mixing period, $\tau_{\mathrm{mix}}$, after which another $90^{\circ}$ pulse is applied and the signal recorded. If atomic rearrangement occurs during $\tau_{\text {mix }}$, a two-dimensional pattern of peaks will result with off-diagonal peaks indicating which sites exchanged magnetization during the mixing time. The phase cycle shown in Figure 2.7 retains mirror-image coherence-transfer pathways to permit the construction of pure-phase two-dimensional spectra (see Section 1.5.2). Spin-temperature
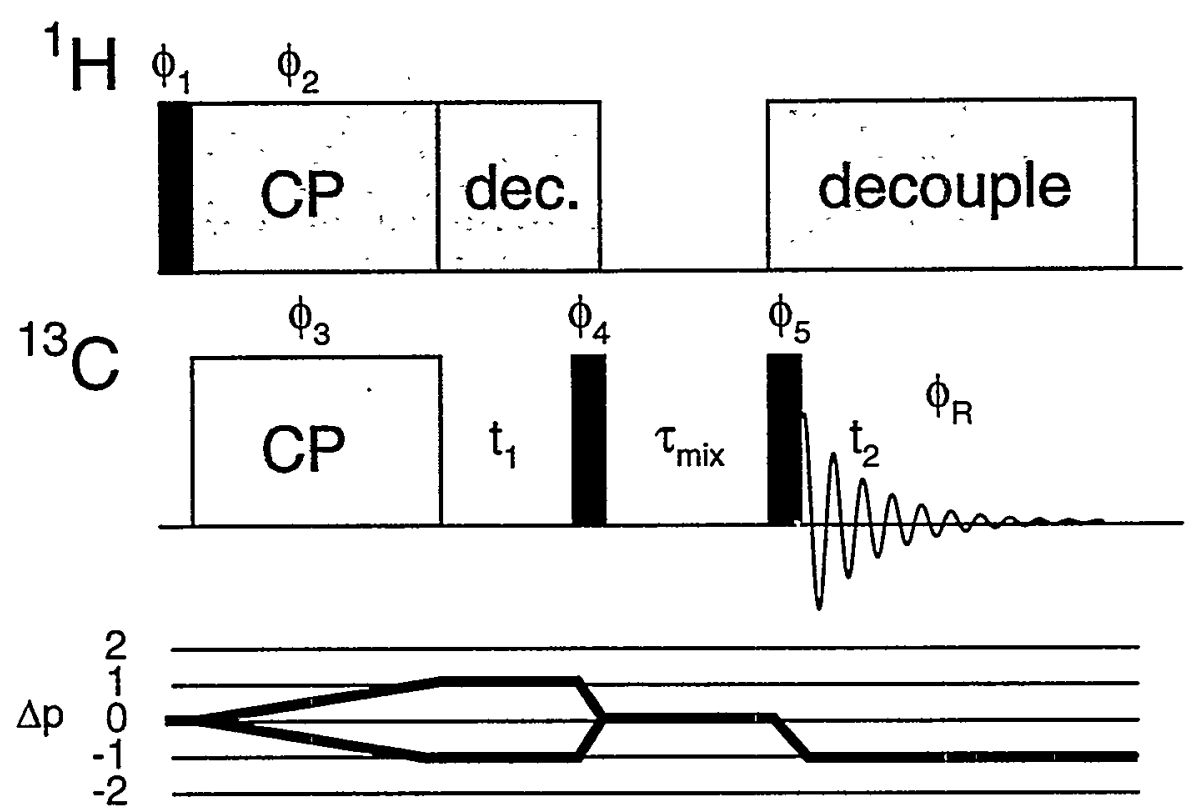

$$
\begin{array}{llll}
\phi_{1}: & 0202 & 0202 \\
\phi_{2}: & 1111 & 1111 \\
\phi_{3}: & 0000 & 0000 & \text { for cosine data set } \\
\phi_{3}: & 1111 & 1111 & \text { for sine data set } \\
\phi_{4}: & 0000 & 0000 & \\
\phi_{5}: & 0011 & 2233 \\
\phi_{R}: & 0213 & 2031 \\
\hline
\end{array}
$$

Figure 2.7 - Pulse sequence, coherence-transfer pathway, and phase cycle for a twodimensional exchange experiment using hypercomplex data acquisition. 
alternation $^{47}$ and CYCLOPS phase cycling ${ }^{46}$ of the last pulse are used to eliminate experimental artifacts. Proton decoupling is used during the acquisition to improve spectral resolution.

Figure 2.8a shows the two-dimensional exchange spectrum of $\mathrm{HfCp}_{4}$ recorded at $188 \mathrm{~K}$ with a mixing time of $50 \mathrm{~ms}$. The experimental apparatus and parameters were the same as those described in Section 2.2. Cross peaks indicated exchange between five pairs of resonances: $(90.0,113.0),(90.0,130.7),(113.0,126.5),(126.5,127.9)$, and $(127.9,130.7)$. Unfortunately, the spectrum was not artifact-free. The $(90.0,113.0)$ and $(113.0,126.5)$ cross peaks were actually more intense than they appear in the contour plot since the two-dimensional spectrum had a negative intensity ridge at $\omega_{2}=113 \mathrm{ppm}$. In addition, there was a positive intensity ridge at $\omega_{1}=113 \mathrm{ppm}$ due to $t_{1}$-noise, an experimental artifact. This obscured two of the cross peaks; however, due to the symmetry of two-dimensional exchange spectroscopy, their presence can he inferred from the other dimension. The quality of the spectrum was not high enough to permit an estimation of the rate constant from the cross peak intensities, but useful information about the rearrangement mechanism could still be obtained.

Several conclusions about peak assignments and the nature of the primary mechanism for rearrangement can be drawn from the locations of the cross peaks in the spectrum. First, the presence of cross peaks between the large peak at $113.0 \mathrm{ppm}$ and some but not all of the monohapto peaks indicates that ring exchange between the monohapto and pentahapto groups cannot be the dominant rearrangement mechanism at this temperature. It also confirms that one of the carbon resonances of the $\eta^{1}-\mathrm{C}_{5} \mathrm{H}_{5}$ group is indeed hidden underneath the $\eta^{5}-\mathrm{C}_{5} \mathrm{H}_{5}$ resonance. Secondly, the presence of cross peaks between the $90.0 \mathrm{ppm}$ resonance, which was assigned to the $\mathrm{C} 1$ carbon (see Section 2.2.2), and the peaks at 113.0 and $130.7 \mathrm{ppm}$ indicates that the primary rearrangement mechanism is not ring flips because the $\mathrm{Cl}$ carbon would not exchange positions during a ring flip. When a longer mixing time (200 ms) was employed to allow multiple exchanges to occur, cross peaks were observed between all of the monohapto resonances (Figure $2.8 \mathrm{~b}$ ). If we assume that significant spin diffusion has not occurred on this timescale (a reasonable assumption for natural abundance ${ }^{13} \mathrm{C}$ in the absence of spin-diffusion driving mechanisms $\left.{ }^{23,107,108}\right)$, this further indicates that the rearrangement mechanism is not ring 

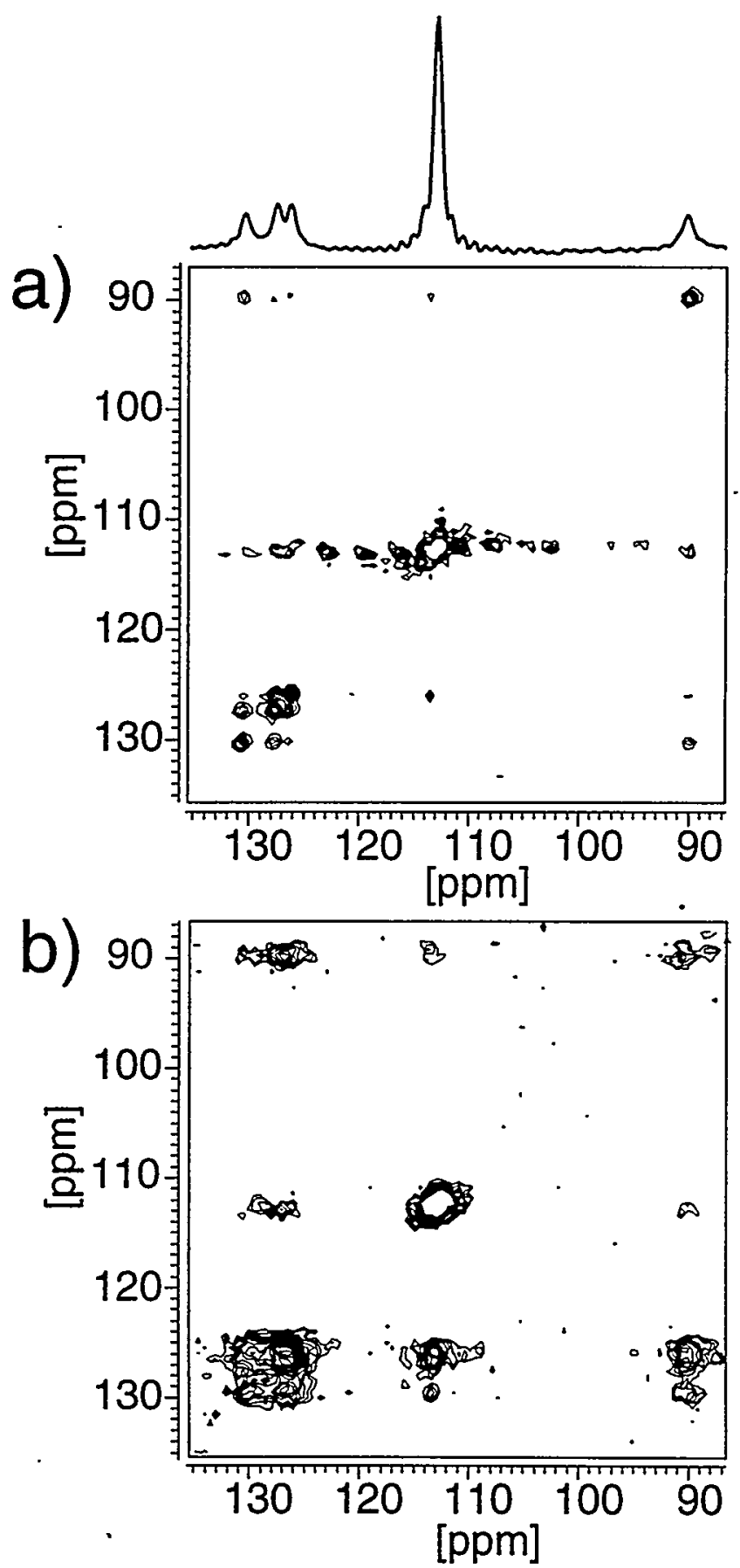

Figure 2.8 - Two-dimensional exchange spectra of $\mathrm{Hf}\left(\eta^{5}-\mathrm{C}_{5} \mathrm{H}_{5}\right)_{2}\left(\eta^{1}-\mathrm{C}_{5} \mathrm{H}_{5}\right)_{2}$ at $188 \mathrm{~K}$. One hundred and twenty-eight $t_{1}$ slices with twenty-four scans in each were recorded with a recycle delay of $3 \mathrm{~s}$. Only positive contours are shown. In (a), a mixing time of $50 \mathrm{~ms}$ was used, while in (b), a $200 \mathrm{~ms}$ mixing time was used. 
flips. Finally, the absence of equal-intensity cross peaks between all of the sites in Figure $2.8 \mathrm{a}$ rules out the possibility of multiple sigmatropic rearrangement mechanisms occurring with nearly equal probability. We can therefore conclude that a single type of sigmatropic rearrangement is the dominant mechanism, and that a single such rearrangement occurs in less than $50 \mathrm{~ms}$ at $188 \mathrm{~K}$.

The next step is to determine whether that mechanism is a $[1,2]$ or a $[1,3]$ sigmatropic rearrangement. Figure 2.9 depicts a schematic of the pattern of cross peaks observed in the two-dimensional exchange spectrum with the $50 \mathrm{~ms}$ mixing time. Unfortunately, interpretation of this pattern requires that the five $\eta^{1}-\mathrm{C}_{5} \mathrm{H}_{5}$ carbon

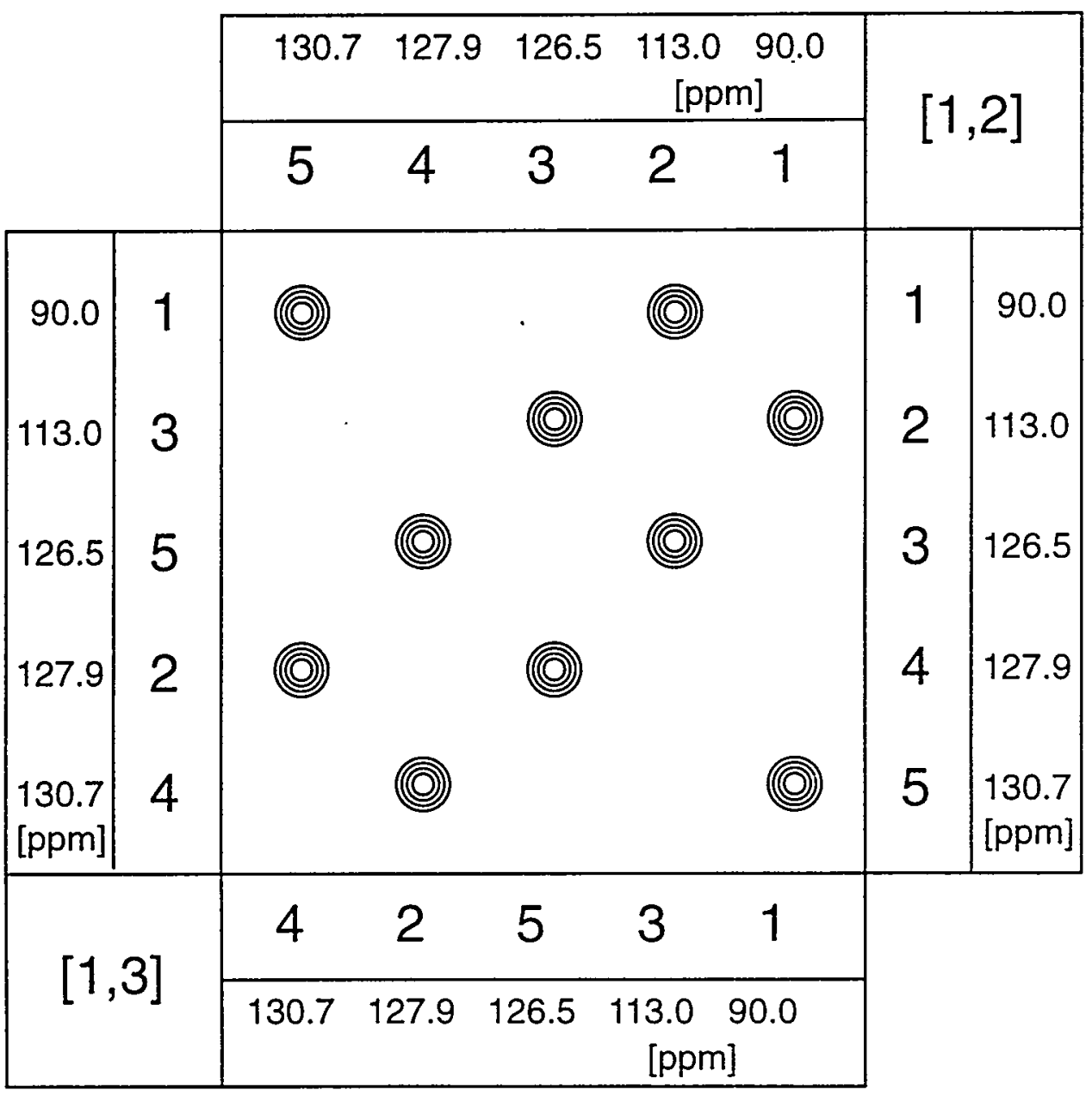

Figure 2.9 - Diagram of the cross peaks observed in the two-dimensional exchange spectrum of $\mathrm{HfCp}_{4}$ and the possible peak assignments. The labels on the top and the right sides of this diagram indicate the carbon resonance assignments that correspond to a $[1,2]$ sigmatropic rearrangement. The labels on the bottom and the left sides of this diagram indicate the carbon resonance assignments that correspond to a $[1,3]$ sigmatropic rearrangement. 
resonances be assigned. This is difficult to do a priori. Although one can assign the 90.0 ppm resonance to the $\mathrm{Cl}$ carbon with reasonable confidence (see Section 2.2.2), assignment of the remaining carbons is more difficult. One can make comparisons with solution-state studies of related compounds, but the perils of such an approach will be discussed in Section 2.4. For $\mathrm{HfCp}_{4}$, two possible assignment schemes are consistent with the experimentally determined pattern of cross peaks. The first, represented by the labels on the top and on the right hand side of Figure 2.9, would assign the $113.0 \mathrm{ppm}$ resonance to the $\mathrm{C} 2$ carbon, the $126.5 \mathrm{ppm}$ resonance to the $\mathrm{C} 3$ carbon, the $127.9 \mathrm{ppm}$ resonance to the $\mathrm{C} 4$ carbon, and the $130.7 \mathrm{ppm}$ resonance to the $\mathrm{C} 5$ carbon. The pattern of cross peaks would then be consistent with a [1,2] sigmatropic rearrangement in which $\mathrm{C} 1 \rightarrow \mathrm{C} 2$, $\mathrm{C} 2 \rightarrow \mathrm{C} 3, \mathrm{C} 3 \rightarrow \mathrm{C} 4, \mathrm{C} 4 \rightarrow \mathrm{C} 5$, and $\mathrm{C} 5 \rightarrow \mathrm{C} 1$. Alternatively, the assignment represented by the labels on the bottom and left sides of Figure 2.9 is possible. In the assignment, the $113.0 \mathrm{ppm}$ resonance would correspond to the $\mathrm{C} 3$ carbon, the $126.5 \mathrm{ppm}$ resonance to the $\mathrm{C} 5$ carbon, the $127.9 \mathrm{ppm}$ resonance to the $\mathrm{C} 2$ carbon, and the $130.7 \mathrm{ppm}$ resonance to the $\mathrm{C} 4$ carbon. The pattern of cross peaks would then be consistent with a $[1,3]$ sigmatropic rearrangement in which $\mathrm{C} 1 \rightarrow \mathrm{C} 3, \mathrm{C} 2 \rightarrow \mathrm{C} 4, \mathrm{C} 3 \rightarrow \mathrm{C} 5, \mathrm{C} 4 \rightarrow \mathrm{C} 1$, and $\mathrm{C} 5 \rightarrow \mathrm{C} 2$.

Knowledge of which NMR peaks were exchanging, even without knowing to which carbons they corresponded, permitted us to reexamine the one-dimensional variable-temperature spectra of Section 2.2.2 and extract Arrhenius parameters. The lineshapes could now be fit using a model in which magnetization hopped among the peaks according to the pattern depicted in Figure 2.9. In these fits, it was assumed that the chemical shifts were constant over the entire temperature range and that the increased line broadening at higher temperatures was entirely due to exchange. It was also assumed that exchange between the centerbands and sidebands was negligible. The monohapto peak at $113.0 \mathrm{ppm}$ could not be fit since it overlapped with the pentahapto peak, but it was assumed to have a similar intensity to the other four monohapto peaks. The relative intensities of the remaining four peaks were extracted from the $133 \mathrm{~K}$ spectrum and fixed to those values in the higher temperature simulations. Figure 2.10 shows the experimental spectra, the simulated spectra, and the rate constants extracted from the fits. 


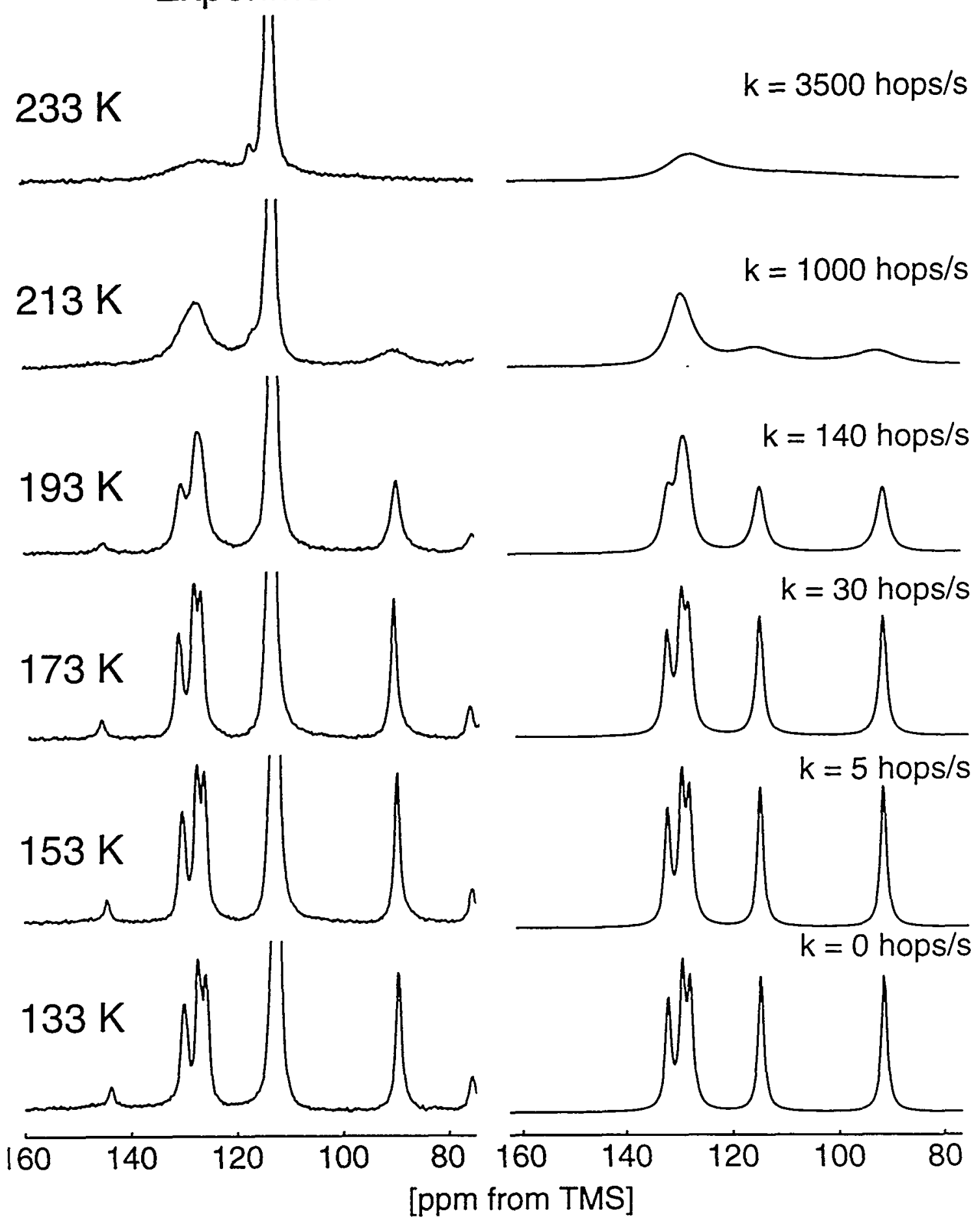

Figure 2.10 - Experimental and simulated spectra corresponding to a single sigmatropic rearrangement of the monohaptocyclopentadienyl groups in $\mathrm{HfCP}_{4}$. The peak at 113.0 $\mathrm{ppm}$ in the experimental spectra is comprised of carbon atoms that are involved in the exchange as well as carbon atoms that are not involved. Only the exchanging carbons are simulated. 
An Arrhenius plot of $\ln (\mathrm{k})$ vs. $1 / \mathrm{T}$ is shown in Figure 2.11 ; the error bars reflect a $\pm 5 \mathrm{~K}$ uncertainty in the temperature. From this plot, the activation energy for the sigmatropic rearrangement that occurs in $\mathrm{HfCp}_{4}$ was found to be $\mathrm{E}_{\mathrm{a}}=24.4 \pm 1.5 \mathrm{~kJ} /(\mathrm{K} \mathrm{mol})$ and the preexponential factor was $\mathrm{A}=8.4 \times 10^{8} \mathrm{~s}^{-1}$.

Once the resonances of the monohapto carbons are reliably assigned, the twodimensional exchange results can be used to definitively determine whether the rearrangement mechanism is a [1,2] or [1,3] shift. To assign the peaks correctly, however, independent experiments (such as INADEQUATE) will need to be performed on a doubly ${ }^{13} \mathrm{C}$-labeled version of $\mathrm{HfCp}_{4}$. Since doubly-labeled cyclopentadienyl rings are difficult to synthesize, such experiments have not yet been performed.

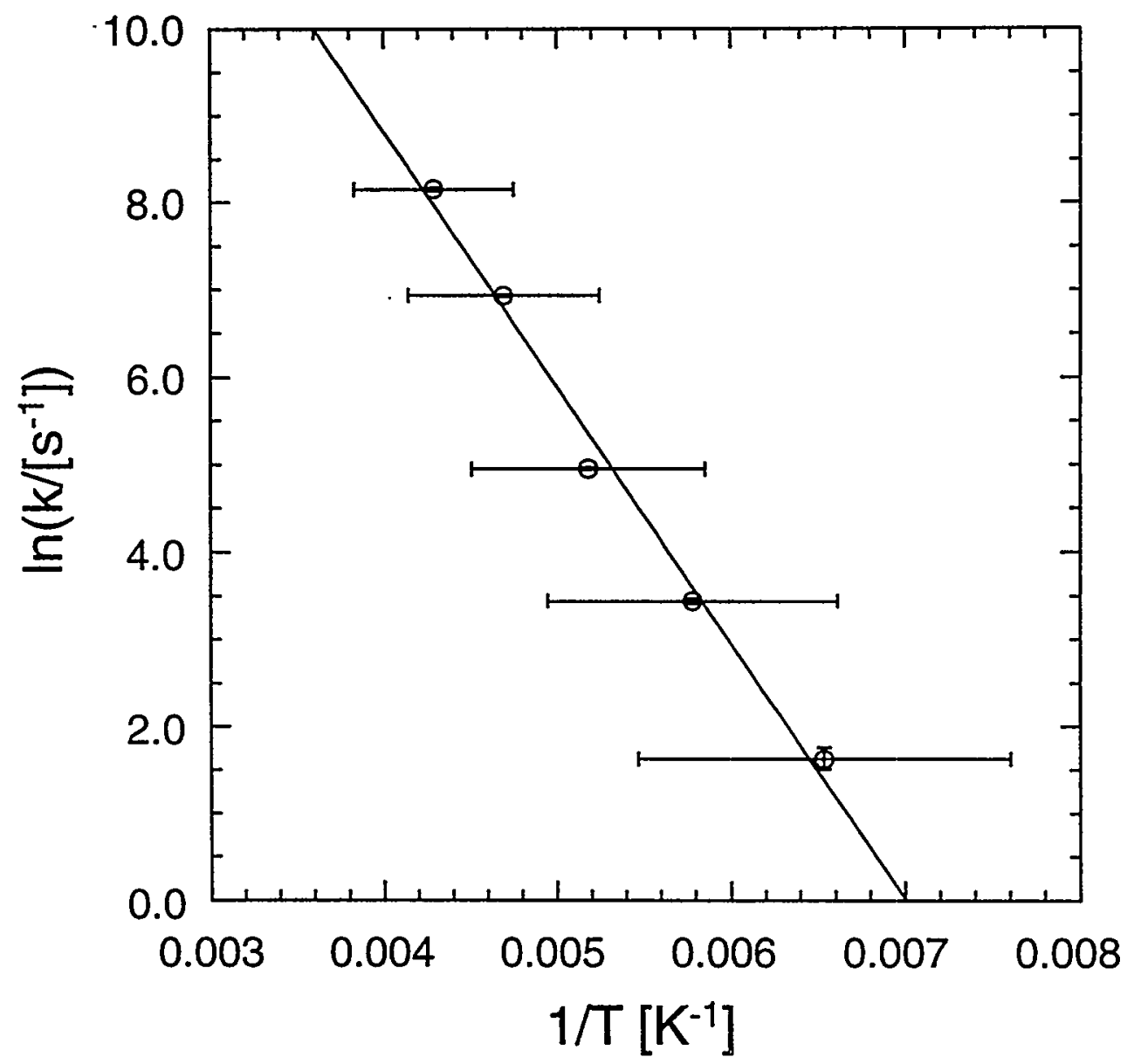

Figure 2.11 - Arrhenius plot of the rate of sigmatropic rearrangement in the monohapto cyclopentadienyl rings of $\mathrm{HfC}_{4}$ in the solid state. The activation energy is $\mathrm{E}_{\mathrm{a}}=24.4 \pm 1.5$ $\mathrm{kJ} /(\mathrm{K} \mathrm{mol})$ and the pre-exponential factor is $\mathrm{A}=8.4 \times 10^{8} \mathrm{~s}^{-1}$. 


\subsection{Comparison of $\mathrm{HfCp}_{4}$ and $\mathrm{TiCp}_{4}$ Exchange Spectra}

Since the crystal structure of $\mathrm{Hf}\left(\eta^{5}-\mathrm{C}_{5} \mathrm{H}_{5}\right)_{2}\left(\eta^{1}-\mathrm{C}_{5} \mathrm{H}_{5}\right)_{2}$ is very similar to that of $\operatorname{Ti}\left(\eta^{5}-\mathrm{C}_{5} \mathrm{H}_{5}\right)_{2}\left(\eta^{1}-\mathrm{C}_{5} \mathrm{H}_{5}\right)_{2}$, it is interesting to compare our results with the results of a recent solid-state NMR study of $\mathrm{TiCp}_{4}{ }^{82}$ In this work, variable-temperature, onedimensional ${ }^{13} \mathrm{C}$ CP/MAS experiments were performed on $\mathrm{TiCp}_{4}$ over the $165-369 \mathrm{~K}$ temperature range. Below $200 \mathrm{~K}$, five distinct monohaptocyclopentadienyl resonances were seen at $89.3,114.9,121.8,126.8$, and 136.3 ppm. A large pentahapto peak at 117.3 ppm was also observed in all spectra. One-dimensional exchange experiments based on magnetization transfer were performed at $165 \mathrm{~K}$, and lineshape analysis of the onedimensional, variable-temperature spectra was used to estimate the Arrhenius parameters for the ring-rearrangement process. The authors of the $\mathrm{TiCp}_{4}$ study concluded that a single dominant sigmatropic rearrangement was the primary motion at $165 \mathrm{~K}$ and that this rearrangement was a $[1,2]$ shift.

While their conclusion that a single sigmatropic rearrangement predominated is supported by their experimental evidence, their method of peak assignment is somewhat suspect. The authors noted that in solution studies of $\mathrm{TiCp}_{4}$ the $\mathrm{C} 2$ resonance is degenerate with the $\mathrm{C} 5$ resonance, and the $\mathrm{C} 3$ resonance is degenerate with the $\mathrm{C} 4$ resonance. $^{98}$ They therefore assumed that in the solid state, the $\mathrm{C} 2$ peak must lie next to the $\mathrm{C} 5$ peak, and the $\mathrm{C} 3$ peak must lie next to the $\mathrm{C} 4$ peak. Although this appears reasonable, our results for the structurally analogous $\mathrm{HfCp}_{4}$ compound show that this is not necessarily the case. The only two peak assignments consistent with the twodimensional exchange spectrum of $\mathrm{HfCP}_{4}$ (see Figure 2.9) require that the peaks in either the $(\mathrm{C} 2, \mathrm{C} 5)$ or the $(\mathrm{C} 3, \mathrm{C} 4)$ pair be separated by $17.7 \mathrm{ppm}$ ! This is, admittedly, quite a large separation for carbons with identical local electronic environments, but as the authors of the $\mathrm{TiCp}_{4}$ study point out, a plausible explanation for large splittings between monohapto carbon resonances in the solid state is a through-space perturbation of the local electronic environment by the aromatic pentahapto rings. ${ }^{82}$ Regardless of the reason for the splittings, it is clear that significant differences between solid and solution-state spectra make assignments based on solution-state studies unreliable. 
The authors of the $\mathrm{TiCp}_{4}$ study continued their arguments for the peak assignments by comparing asymmetry parameters for the different sites and discussing expected ring current shifts. They did not, however, justify why they thought the asymmetry parameter was a valid criterion for assigning pairs of carbon resonances within monohaptocyclopentadienyl groups. The components of the chemical-shielding tensor can be quite sensitive to long-range interactions, ${ }^{101}$ and the asymmetry parameters for the monohapto carbons in $\mathrm{TiCp}_{4}(\eta=0.54,0.83,0.87,0.72$, and 0.74 ) were significantly different from those measured in the $\mathrm{HfCp}_{4}$ analogue (see Table 2.1).

The final assignments made by the authors of the $\mathrm{TiCp}_{4}$ study were the following: $\mathrm{C} 1=89.3 \mathrm{ppm}, \mathrm{C} 3=114.9 \mathrm{ppm}, \mathrm{C} 4=121.8 \mathrm{ppm}, \mathrm{C} 2=126.8 \mathrm{ppm}$, and $\mathrm{C} 5=136.3 \mathrm{ppm}$. These assignments were then used in combination with magnetization-transfer experiments and with lineshape analysis to infer that a [1,2] sigmatropic shift rearrangement was the dominant mechanism for the monohaptocyclopentadienyl rings in $\mathrm{TiCp}_{4}$ at $165 \mathrm{~K}$.

Although it may be tempting to make a one-to-one correspondence between the peaks in $\mathrm{TiCp}_{4}$ and those in $\mathrm{HfCp}_{4}$, the above discussion shows that such assumptions can be dangerous. Despite their similar structures, $\mathrm{HfCP}_{4}$ and $\mathrm{TiCp}_{4}$ have different crystalpacking geometries, and the precise effect of such differences on the chemical shift is unknown. We therefore chose to perform the same two-dimensional exchange experiment (see Figure 2.7) directly on $\mathrm{TiCp}_{4}$.

A sample of $\mathrm{TiCp}_{4}$ was synthesized by Dr. Murray S. Davies according to the method of Calderon et al. ${ }^{98}$ Due to the extreme sensitivity of this compound to moisture and oxygen, the sample was sealed in a Wilmad glass rotor insert, and the entire insert was placed into a $7.5 \mathrm{~mm}$ zirconia rotor. The two-dimensional experiment was performed by Professor Eric Munson and Michelle Douskey at the University of Minnesota on a similar apparatus to that described in Section 2.1 .

Figure 2.12 shows the two-dimensional exchange spectrum of $\mathrm{TiCp}_{4}$ recorded at $183 \mathrm{~K}$ with a mixing time of $50 \mathrm{~ms}$. Comparison with Figure 2.8 reveals that the same pattern of cross peaks is present in both $\mathrm{HfCp}_{4}$ and $\mathrm{TiC}_{4}$. Therefore, by the arguments of Section 2.3, only two sets of peak assignments are possible. In one assignment, consistent with a [1,2] shift, the $114.9 \mathrm{ppm}$ resonance corresponds to the $\mathrm{C} 2$ carbon, the $121.8 \mathrm{ppm}$ 
resonance to the $\mathrm{C} 3$ carbon, the $126.8 \mathrm{ppm}$ resonance to the $\mathrm{C} 4$ carbon, and the $136.3 \mathrm{ppm}$ resonance to the $\mathrm{C} 5$ carbon. For the other assignment, consistent with a $[1,3] \mathrm{shift}$, the $114.9 \mathrm{ppm}$ resonance corresponds to the $\mathrm{C} 3$ carbon, the $121.8 \mathrm{ppm}$ resonance to the $\mathrm{C} 5$ carbon, the $126.8 \mathrm{ppm}$ resonance to the $\mathrm{C} 2$ carbon, and the $136.3 \mathrm{ppm}$ resonance to the $\mathrm{C} 4$ carbon. The assignment proposed by the authors of the $\mathrm{TiCp}_{4}$ study cannot, therefore, be correct. As in the case of $\mathrm{HfCp}_{4}$, independent experiments will be required to determine whether a $[1,2]$ or $[1,3]$ sigmatropic rearrangement mechanism is occurring.

This example highlights the utility of two-dimensional exchange spectroscopy. Unlike one-dimensional experiments, which require one to make a series of assumptions, the two-dimensional experiments provide direct evidence of which sites are exchanging.

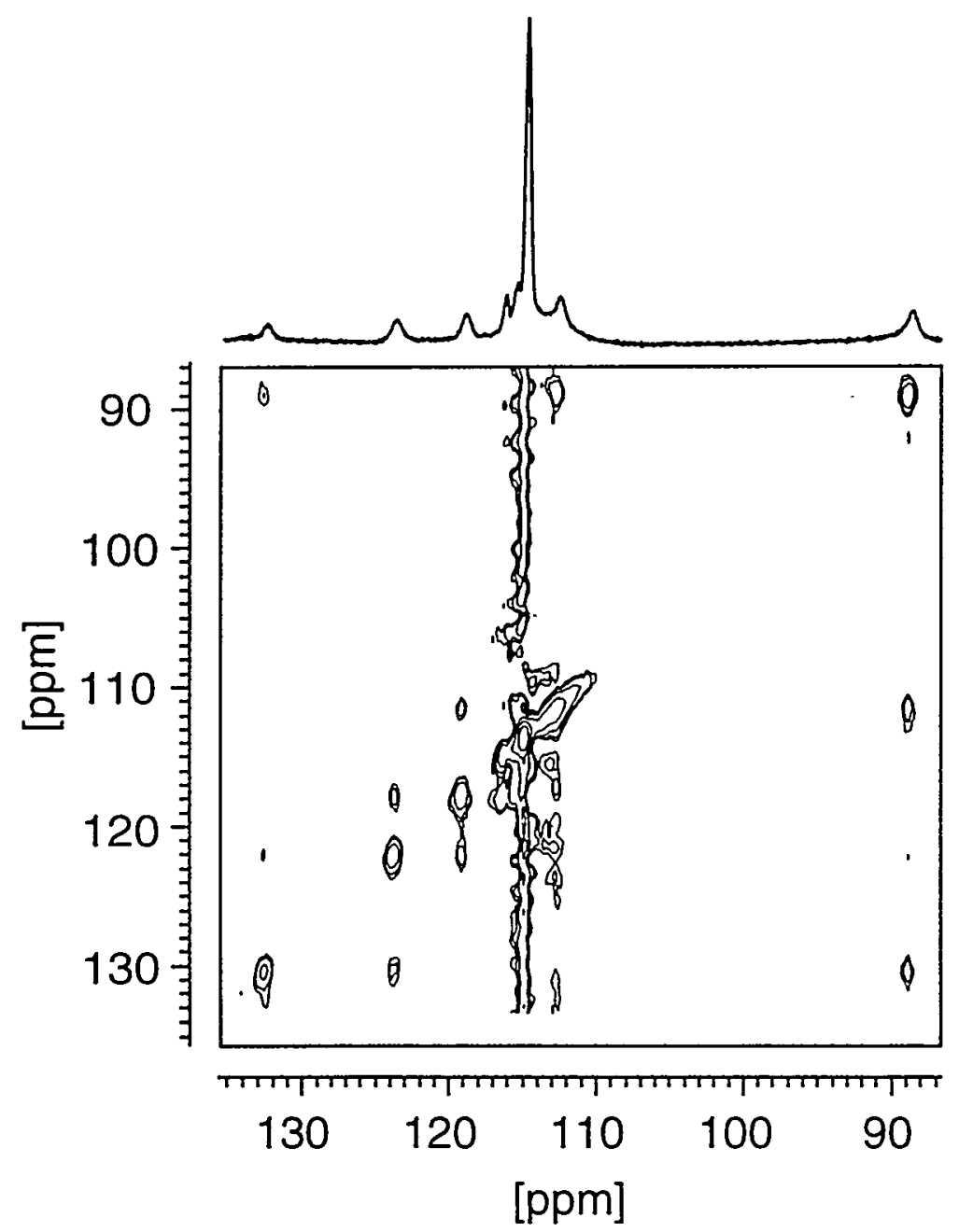

Figure 2.12 - Two-dimensional exchange spectrum of Ti $\left(\eta^{5}-\mathrm{C}_{5} \mathrm{H}_{5}\right)_{2}\left(\eta^{1}-\mathrm{C}_{5} \mathrm{H}_{5}\right)_{2}$ at $183 \mathrm{~K}$. Thirty-two $t_{1}$ slices were recorded. Only positive contours are shown. The mixing time was $50 \mathrm{~ms}$. 
For the cases of $\mathrm{HfCp}_{4}$ and $\mathrm{TiCp}_{4}$, they have shown that the monohaptocyclopentadienyl rings predominantly undergo a single type of sigmatropic rearrangement in the solid state and that only two sets of peak assignments are possible. When combined with an independent measurement of the connectivities between neighboring carbons (for instance, in an INADEQUATE experiment), the exchange spectra will enable the ringrearrangement mechanism to be definitively determined. 


\section{Chapter 3: Spin Locking of Quadrupolar Nuclei During MAS}

All of the experiments presented in Chapter 2 required cross polarization from ${ }^{1} \mathrm{H}$ to ${ }^{13} \mathrm{C}$ to enhance the carbon sensitivity. Without such cross polarization, the carbon signal would have been too weak to be detected on a practical timescale. The favorable properties of protons -- a high gyromagnetic ratio, high natural abundance, and typically short longitudinal relaxation times -- have made their use as a polarization source nearly ubiquitous in studies of organic, organometallic, and biological systems.

However, many interesting inorganic materials lack protons. In such cases, another polarization source is necessary to facilitate the study of insensitive nuclei. While nuclei such as ${ }^{23} \mathrm{Na}$ and ${ }^{27} \mathrm{Al}$ seem promising due to their $100 \%$ natural abundance and short relaxation times, they have spin-quantum numbers greater than $1 / 2$ and therefore are subject to the quadrupolar interaction. This can create complications when attempting to cross polarize from them.

Since cross polarization can only occur if both spins can be spin-locked long enough for magnetization to be transferred, it is necessary to determine the conditions under which efficient spin locking is possible. In this chapter, the complications inherent in spin locking a quadrupolar nucleus will be examined by both experiments and simulations on a model aluminosilicate compound.

\subsection{Low Albite as a Model Compound}

Silicon is a major component of many technologically and geochemically important inorganic materials including zeolites, glasses, minerals, and gels. Since these materials often have limited long-range order, solid-state ${ }^{29} \mathrm{Si}$ NMR has proven to be particularly useful in eliciting information about their structure. ${ }^{109,110}$ However, the low natural abundance of ${ }^{29} \mathrm{Si}$ (4.7\%) combined with its relatively low gyromagnetic ratio make ${ }^{29} \mathrm{Si}$ NMR inherently insensitive. Furthermore, significant improvement of the signal-to-noise ratio by signal averaging is usually time-consuming due to the typically long $\mathrm{T}_{1}$ relaxation times of ${ }^{29} \mathrm{Si}$ (frequently on the order of minutes). Consequently, twodimensional experiments are often impractical, unless isotopic enrichment or cross 
polarization $(\mathrm{CP})^{111}$ is used. If the silicate contains protons, cross polarization from protons to silicon can greatly enhance the ${ }^{29} \mathrm{Si} \mathrm{NMR}$ intensities. In this thesis, however, the case where protons are not present is investigated to see if cross polarization from quadrupolar nuclei can provide a significant enhancement of the ${ }^{29} \mathrm{Si}$ sensitivity.

Figure 3.1 depicts the crystal structure of the feldspar low albite $\left(\mathrm{NaAlSi}_{3} \mathrm{O}_{8}\right)$ as determined by $\mathrm{X}$-ray and neutron diffraction. ${ }^{112}$ Low albite was chosen as a model compound for ${ }^{27} \mathrm{Al}-$ to- $^{29} \mathrm{Si}$ and ${ }^{23} \mathrm{Na}$-to- ${ }^{29} \mathrm{Si}$ cross-polarization experiments for several reasons. In many ways low albite is a typical inorganic aluminosilicate, composed of a framework of connected $\mathrm{AlO}_{4}$ and $\mathrm{SiO}_{4}$ tetrahedra and non-framework, charge-balancing counterions (in this case $\mathrm{Na}^{+}$). ${ }^{113}$ However, the analysis of cross polarization from the quadrupolar nuclei in low albite is simplified by the presence of only one crystallographic ${ }^{27} \mathrm{Al}$ site and one ${ }^{23} \mathrm{Na}$ site. Furthermore, low albite is highly ordered with the silicon occupying three distinct crystallographic T-sites in equal amounts. ${ }^{112}$ Two of these sites are coordinated via bridging oxygens to one aluminum atom and three silicon atoms and are commonly denoted as $\mathrm{Q}^{4}(1 \mathrm{Al})$ sites. The superscript 4 indicates that all four oxygens are bridging, and the integer 1 indicates the presence of a single aluminum "nearest neighbor." The third silicon site is coordinated via bridging oxygens to two aluminum and

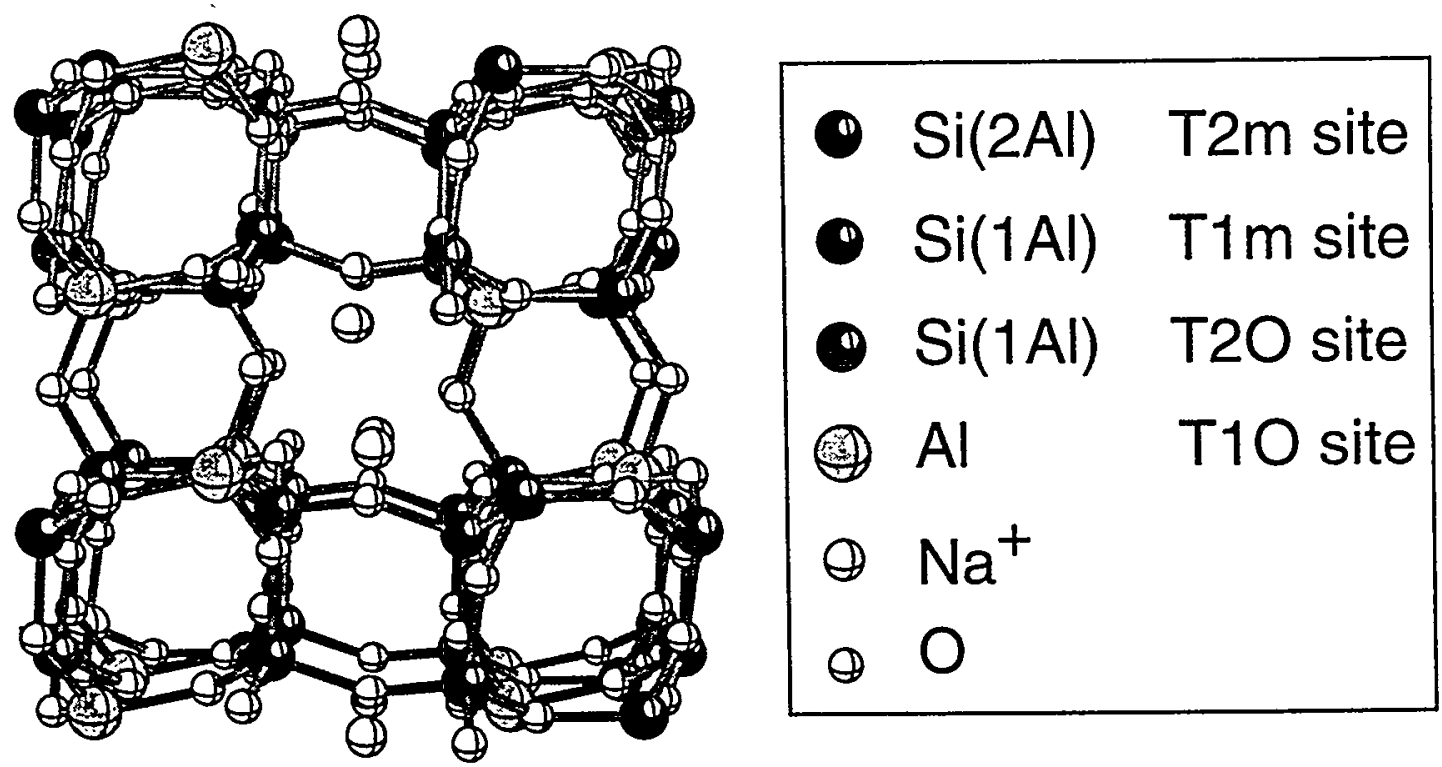

Figure 3.1 - The structure of low albite $\left(\mathrm{NaAlSi}_{3} \mathrm{O}_{8}\right)$ as determined by $\mathrm{X}$-ray and neutron diffraction. Four crystallographically inequivalent tetrahedral sites are present: one is occupied exclusively by $\mathrm{Al}$ and the other three by $\mathrm{Si}$ atoms. 
two silicon atoms and is denoted as a $\mathrm{Q}^{4}(2 \mathrm{Al})$ site. Assignment of these silicon sites to three distinct and narrow ${ }^{29} \mathrm{Si}$ MAS NMR resonances can be found in the literature. ${ }^{113,114,115,116,117}$ (Note that there is a mistake in the labeling of the crystallographic sites in Ref. ${ }^{116}$.) Table 3.1 lists these assignments along with the distances between each

\begin{tabular}{|c|c|c|c|c|}
\hline $\begin{array}{c}\text { Isotropic } \\
\text { Shift } \\
\text { [ppm] }\end{array}$ & $\begin{array}{c}\text { Crystallographic } \\
\text { T-site }\end{array}$ & $\begin{array}{c}\text { Number of } \\
\text { aluminum } \\
\text { nearest } \\
\text { neighbors }\end{array}$ & $\begin{array}{c}\text { Distance to } \\
\text { aluminum } \\
\text { nearest } \\
\text { neighbor(s) }\end{array}$ & $\begin{array}{c}\text { Distance to } \\
\text { nearest } \\
\text { sodium }^{\mathbf{b}}\end{array}$ \\
\hline \hline-91.8 & $\mathrm{~T} 2 \mathrm{~m}$ & 2 & $\begin{array}{c}3.019 \AA \\
3.080 \AA\end{array}$ & $3.291 \AA$ \\
\hline-96.1 & $\mathrm{~T} 20$ & 1 & $3.132 \AA$ & $3.494 \AA$ \\
\hline-103.9 & $\mathrm{~T} 1 \mathrm{~m}$ & 1 & $3.156 \AA$ & $3.394 \AA$ \\
\hline
\end{tabular}

Table 3.1 - Silicon sites in low albite $\left(\mathrm{NaAlSi}_{3} \mathrm{O}_{8}\right)$.

a. Chemical shift values were referenced to an external TMS standard and are within 1 ppm of literature values. ${ }^{113,114,115,116,117}$

b. Distances were determined using neutron-diffraction data from Harlow et al. ${ }^{112}$

silicon site and its nearest aluminum and sodium neighbors. The ${ }^{29} \mathrm{Si}$ isotropic chemical shifts follow the typical trends for aluminosilicates with more aluminum nearest neighbors corresponding to more deshielding within a given $\mathrm{Q}^{\mathrm{n}}$ group. ${ }^{110}$ The sample of low albite used for the experiments in this thesis came from Cazadero, California, U.S.A. Figure 3.2 shows the one-dimensional ${ }^{29} \mathrm{Si}$ MAS spectrum of this sample. Although other researchers have reported seeing splittings in two of the ${ }^{29} \mathrm{Si}$ resonances in low albite

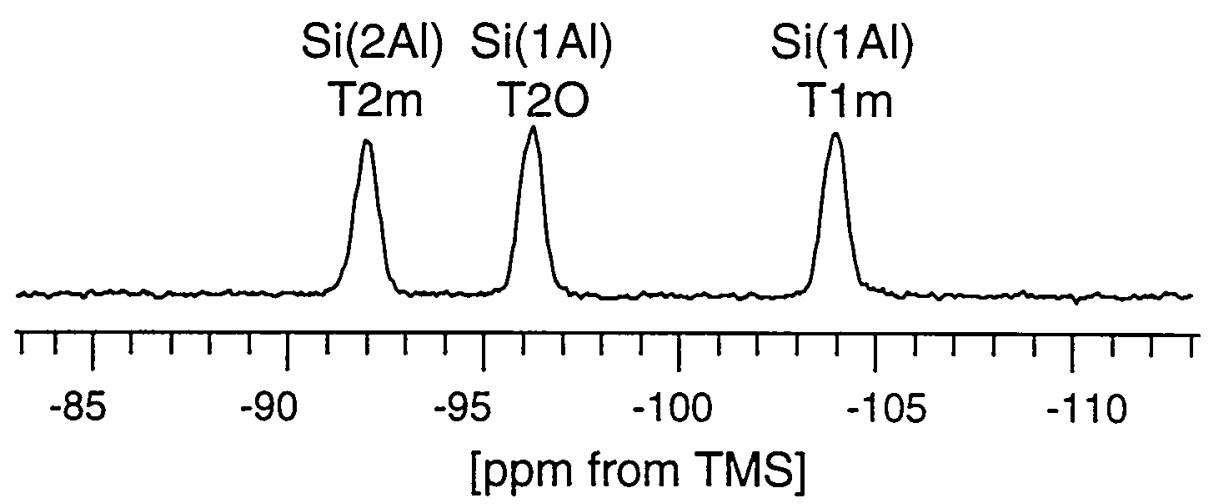

Figure 3.2 $-{ }^{29} \mathrm{Si}$ MAS NMR spectrum of low albite recorded at $11.7 \mathrm{~T}$ with a spinning speed of $2.4 \mathrm{kHz}$ and a recycle delay of $2000 \mathrm{~s}$. 
(which they attributed to heteronuclear J-couplings of approximately 8-9 $\mathrm{Hz}$ in strength), ${ }^{118}$ no such splittings were observed in our experiments. However, magneticfield inhomogeneity (due to sub-optimal shimming) may have obscured the J couplings.

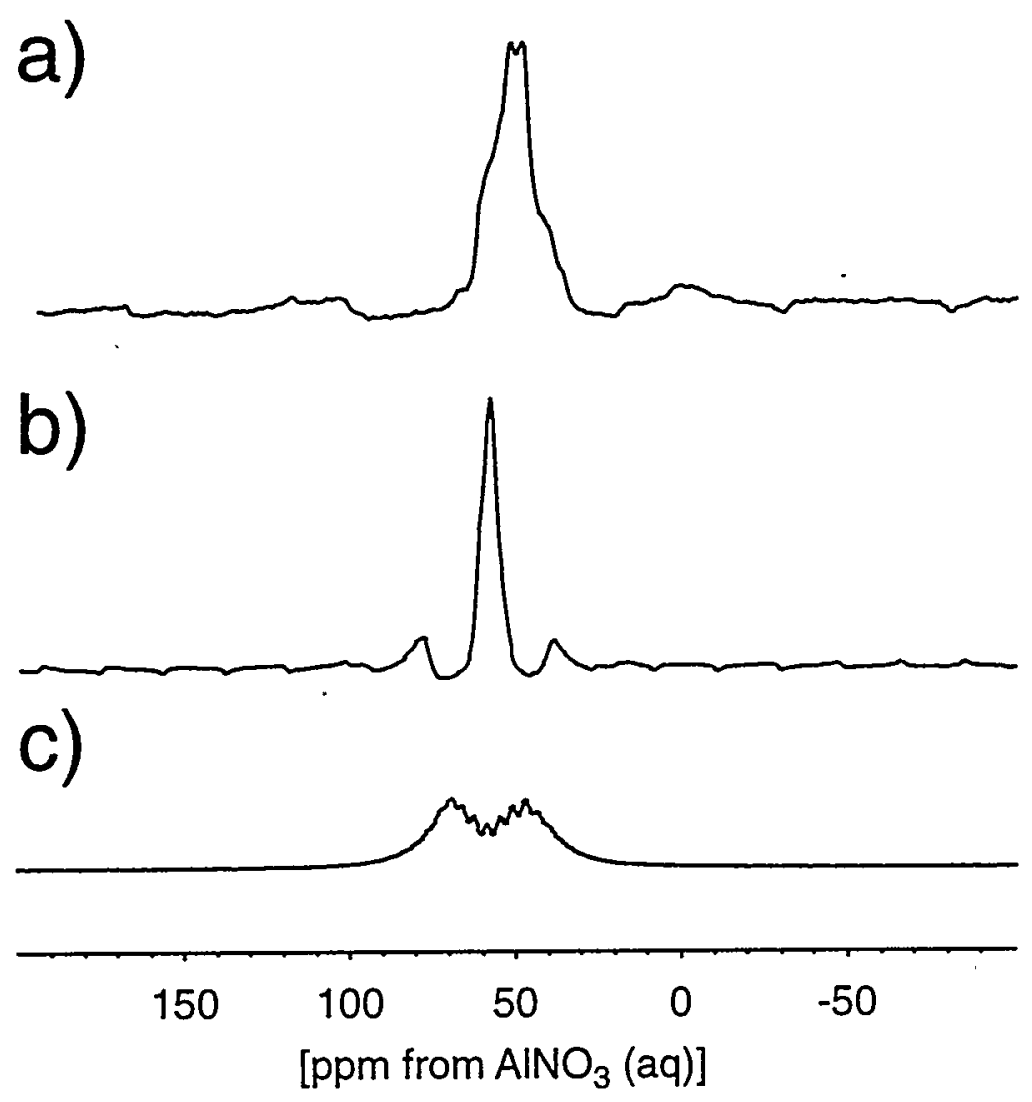

Figure $3.3-{ }^{27} \mathrm{Al}$ MAS spectra of low albite at (a) $9.4 \mathrm{~T}, 4 \mathrm{kHz}$ spinning speed, (b) $11.7 \mathrm{~T}$, $2.4 \mathrm{kHz}$ spinning speed, and (c) $11.7 \mathrm{~T}, 500 \mathrm{~Hz}$ spinning speed. To a good approximation only the central transition is excited and detected.

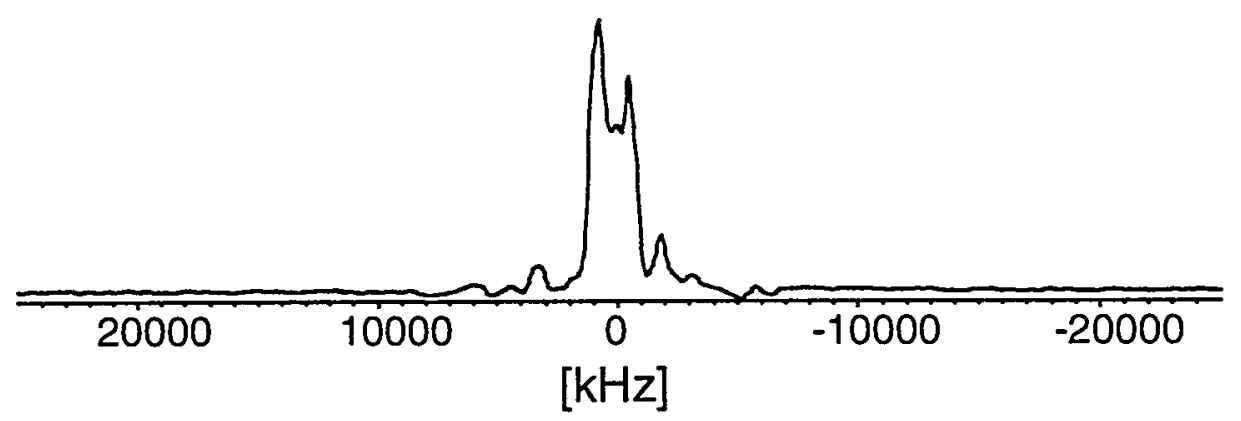

Figure 3.4 - ${ }^{23} \mathrm{Na}$ MAS spectra of low albite at $11.7 \mathrm{~T}, 2.4 \mathrm{kHz}$ spinning speed. Only the central transition is excited/detected. 
The ${ }^{27} \mathrm{Al}$ and ${ }^{23} \mathrm{Na}$ MAS spectra of low albite at various field strengths and spinning speeds are shown in Figures 3.3 and 3.4. The ${ }^{27} \mathrm{Al}$ spectrum is relatively structureless at $11.7 \mathrm{~T}$ and a spinning speed of $2.4 \mathrm{kHz}$, but the ${ }^{23} \mathrm{Na}$ spectrum shows a more typical powder pattern under the same conditions. Several sets of quadrupolar parameters for these sites have been published in the literature ${ }^{113,118.119}$ and are summarized in Table 3.2 .

\begin{tabular}{|c|c|c|c|}
\hline Nucleus & $\mathrm{C}_{\mathrm{qcc}}[\mathrm{MHz}]$ & $\eta$ & Reference \\
\hline \multirow[t]{3}{*}{${ }^{27} \mathrm{Al}$} & 3.37 & 0.634 & Brun et al. ${ }^{119}$ \\
\hline & 3.29 & 0.62 & Kirkpatrick et al. ${ }^{11}$ \\
\hline & 3.32 & 0.64 & Woessner et al. ${ }^{118}$ \\
\hline \multirow[t]{2}{*}{${ }^{23} \mathrm{Na}$} & 2.62 & 0.25 & Brun et al. ${ }^{119}$ \\
\hline & 2.59 & 0.25 & Kirkpatrick et al. ${ }^{11}$ \\
\hline
\end{tabular}

Table 3.2 - Quadrupolar parameters in low albite $\left(\mathrm{NaAlSi}_{3} \mathrm{O}_{8}\right)$

\subsection{Spin Locking of Half-Integer Quadrupolar Nuclei}

Since cross polarization can only occur if both spins can be spin-locked long enough for magnetization to be transferred, it is necessary to determine the conditions under which efficient spin locking is possible. Both spin-1/2 and quadrupolar nuclei undergo relaxation during a spin lock, characterized by one or several rotating-frame relaxation time constants, $T_{1 \rho}$. However, the behavior of the central transition of a halfinteger quadrupolar nucleus during a spin lock is also influenced by the time dependence of the quadrupolar coupling under MAS, which can dramatically reduce the spin-locking efficiency for certain combinations of rf field strengths, spinning speeds, and quadrupolar coupling constants. A theoretical treatment of some of these interference effects has been given in the literature ${ }^{120,121,122,123}$ and will be summarized here. Throughout this chapter and the next, the S-spin refers to the quadrupolar nucleus $\left({ }^{27} \mathrm{Al}\right.$ or ${ }^{23} \mathrm{Na}$ ) and the I-spin to the spin- $1 / 2$ nucleus $\left({ }^{29} \mathrm{Si}\right)$.

When a spin-lock field is applied to a quadrupolar nucleus, the rotating-frame Hamiltonian can be written (in units of energy) as 


$$
\tilde{\mathcal{H}}=\hbar \omega_{1 \mathrm{~S}} \mathrm{~S}_{\mathrm{x}}+\hbar \Delta \omega \mathrm{S}_{\mathrm{z}}+\tilde{\mathcal{H}}_{\mathrm{Q}}
$$

where $\omega_{1 S}$ is strength of the rf-field used for the spin lock, $\Delta \omega$ is the offset of the irradiation from the Larmor frequency, and $\tilde{H}_{Q}$ is the rotating-frame quadrupolar Hamiltonian. The quadrupolar Hamiltonian in the rotating frame can be expressed in terms of irreducible spherical tensor operators (see Section 1.2.5), ${ }^{6}$ and an operator form of static perturbation theory can then be applied. ${ }^{28}$ This procedure gives a first-order term

$$
\tilde{\mathcal{H}}_{\mathrm{Q}}^{(1)}=\hbar\left(\frac{\omega_{\mathrm{Q}}}{3}\right) \mathrm{R}_{2,0}^{\mathrm{Q}} \mathrm{T}_{2,0}^{\mathrm{Q}}
$$

where $\mathrm{R}_{2,0}^{\mathrm{Q}}$ and $\mathrm{T}_{2,0}^{\mathrm{Q}}$ are spatial and spin tensors, respectively, ${ }^{6}$ and $\omega_{\mathrm{Q}}$ is the quadrupolar coupling constant

$$
\omega_{Q}=\frac{3 \mathrm{e}^{2} \mathrm{qQ}}{2 \mathrm{~S}(2 \mathrm{~S}-1) \hbar}=\frac{6 \pi}{2 \mathrm{~S}(2 \mathrm{~S}-1)} \mathrm{C}_{\mathrm{qcc}} .
$$

The second-order contribution to the quadrupolar Hamiltonian is

$$
\tilde{\mathcal{H}}_{\mathrm{Q}}^{(2)}=\frac{\hbar \omega_{\mathrm{Q}}^{2}}{18 \omega_{\mathrm{L}}}\left\{2 \mathrm{R}_{2,1}^{\mathrm{Q}} \mathrm{R}_{2,-1}^{\mathrm{Q}}\left[\mathrm{T}_{2,1}^{\mathrm{Q}}, \mathrm{T}_{2,-1}^{\mathrm{Q}}\right]+\mathrm{R}_{2,2}^{\mathrm{Q}} \mathrm{R}_{2,-2}^{\mathrm{Q}}\left[\mathrm{T}_{2,2}^{\mathrm{Q}}, \mathrm{T}_{2,-2}^{\mathrm{Q}}\right]\right\}
$$

where $\omega_{\mathrm{L}}$ is the Larmor frequency.

Because the $R_{2, m}^{Q}$ terms in Equations (3.2) and (3.4) are orientation-dependent, the pattern of energy level spacings for a quadrupolar nucleus under a spin lock differs for different orientations of a crystallite with respect to the static magnetic field. This creates complications when a sample is spun about an angle other than $0^{\circ}$ since the spinning process changes the orientations of the crystallites in a powder sample. ${ }^{124}$ Considering just the first-order term of the quadrupolar Hamiltonian and applying the Wigner rotation matrices as shown in Section 1.4.2, one can write Equation (3.2) as 


$$
\begin{aligned}
\tilde{H}_{\mathrm{Q}}^{(1)}(\mathrm{t})=\hbar \mathrm{T}_{2,0}^{\mathrm{Q}} \cdot \sum_{\mathrm{m}=-2}^{2}\left\{( \frac { \omega _ { \mathrm { Q } } } { 3 } ) \mathrm { d } _ { - \mathrm { m } , 0 } ^ { ( 2 ) } ( \theta ) \mathrm { e } ^ { \mathrm { im } \omega _ { \mathrm { r } } \mathrm { t } } \cdot \left[\sqrt{\frac{3}{2}} \mathrm{D}_{0,-\mathrm{m}}^{(2)}\left(\alpha^{\mathrm{Q}}, \beta^{\mathrm{Q}}, \gamma^{\mathrm{Q}}\right)\right.\right. \\
\left.\left.\quad+\frac{1}{2} \eta^{\mathrm{Q}}\left(\mathrm{D}_{-2,-\mathrm{m}}^{(2)}\left(\alpha^{\mathrm{Q}}, \beta^{\mathrm{Q}}, \gamma^{\mathrm{Q}}\right)+\mathrm{D}_{2,-\mathrm{m}}^{(2)}\left(\alpha^{\mathrm{Q}}, \beta^{\mathrm{Q}}, \gamma^{\mathrm{Q}}\right)\right)\right]\right\} \\
=\mathrm{T}_{2,0}^{\mathrm{Q}} \cdot \hbar \Omega^{(1 \mathrm{Q})}\left(\alpha^{\mathrm{Q}}, \beta^{\mathrm{Q}}, \gamma^{\mathrm{Q}}, \mathrm{t}\right) .
\end{aligned}
$$

Here, $\alpha^{\mathrm{Q}}, \beta^{\mathrm{Q}}$, and $\gamma^{\mathrm{Q}}$ are Euler angles relating the principal-axis system of the quadrupolar interaction in a given crystallite to the reference frame of a rotor spinning about an axis oriented at an angle $\theta$ relative to the static magnetic field. For $\theta=0^{\circ}$, we see from Table 1.1 that

$$
\mathrm{d}_{-\mathrm{m} 0}^{(2)}\left(0^{\circ}\right)=\left\{\begin{array}{l}
1 \text { for } m=0 \\
0 \text { for } m=-2,-1,1,2
\end{array} .\right.
$$

Thus only the time-independent $(\mathrm{m}=0)$ term of Equation (3.5) remains, and it is clearly seen that spinning about $0^{\circ}$ is equivalent to not spinning at all. For $\theta=54.74^{\circ}$ (the magic angle), $\mathrm{d}_{0,0}^{(2)}\left(54.74^{\circ}\right)=0$, but the $\mathrm{m} \neq 0$ terms are non-zero. All first-order quadrupolar terms are, therefore, time-dependent under MAS, and the sign of $\Omega^{(1 \mathrm{Q})}\left(\alpha^{\mathrm{Q}}, \beta^{\mathrm{Q}}, \gamma^{\mathrm{Q}}, \mathrm{t}\right)$ can change zero, two, or four times per rotor cycle. Figure 3.5 shows an energy-level diagram for the Hamiltonian of Equation (3.1) where $S=5 / 2$ and where only the first-order contribution to $\tilde{\mathcal{H}}_{\mathrm{Q}}$ is considered. The eigenvalues are plotted as a function of $\Omega^{(1 Q)} / \omega_{1 S}$. When $\left|\Omega^{(1 Q)} / \omega_{1 S}\right|$ is large, the eigenstates of Equation (3.1) are welldefined and are labeled along each side of Figure 3.5. Four of these eigenstates are eigenvectors of $S_{z}$. The remaining two eigenstates, $\left|c^{+}\right\rangle$and $\left|c{ }^{-}\right\rangle$, are eigenstates of the fictitious spin- $1 / 2$ operator ${ }^{4}$ on the central transition $S_{x}^{(3,4)}$ and are defined as

$$
\begin{aligned}
& \left|c^{+}\right\rangle=\frac{1}{\sqrt{2}}\left\{\left|\frac{1}{2}\right\rangle+\left|-\frac{1}{2}\right\rangle\right\} \\
& \left|c^{-}\right\rangle=\frac{1}{\sqrt{2}}\left\{\left|\frac{1}{2}\right\rangle-\left|-\frac{1}{2}\right\rangle\right\}
\end{aligned}
$$


For small values of $\left|\Omega^{(1 Q)} / \omega_{1 S}\right|$, the eigenstates of the Hamiltonian are linear combinations of these six eigenstates. A similar diagram can be drawn for the spin-3/2 case (see Figure 3.6).

Note that the ordering of the eigenstates in Figures 3.5 and 3.6 depends on whether $\Omega^{(1 Q)}$ is positive or negative. Of particular interest, therefore, is the issue of what can happen to the spin-state populations when $\Omega^{(1 Q)}$ changes sign (i.e. - undergoes a "zero crossing") and how this influences the efficiency of the spin lock. To characterize the possible scenarios, an adiabaticity parameter, $\alpha$, has been defined ${ }^{120,121,125}$

$$
\alpha=\frac{\omega_{1 S}^{2}}{\omega_{Q} \omega_{r}}
$$

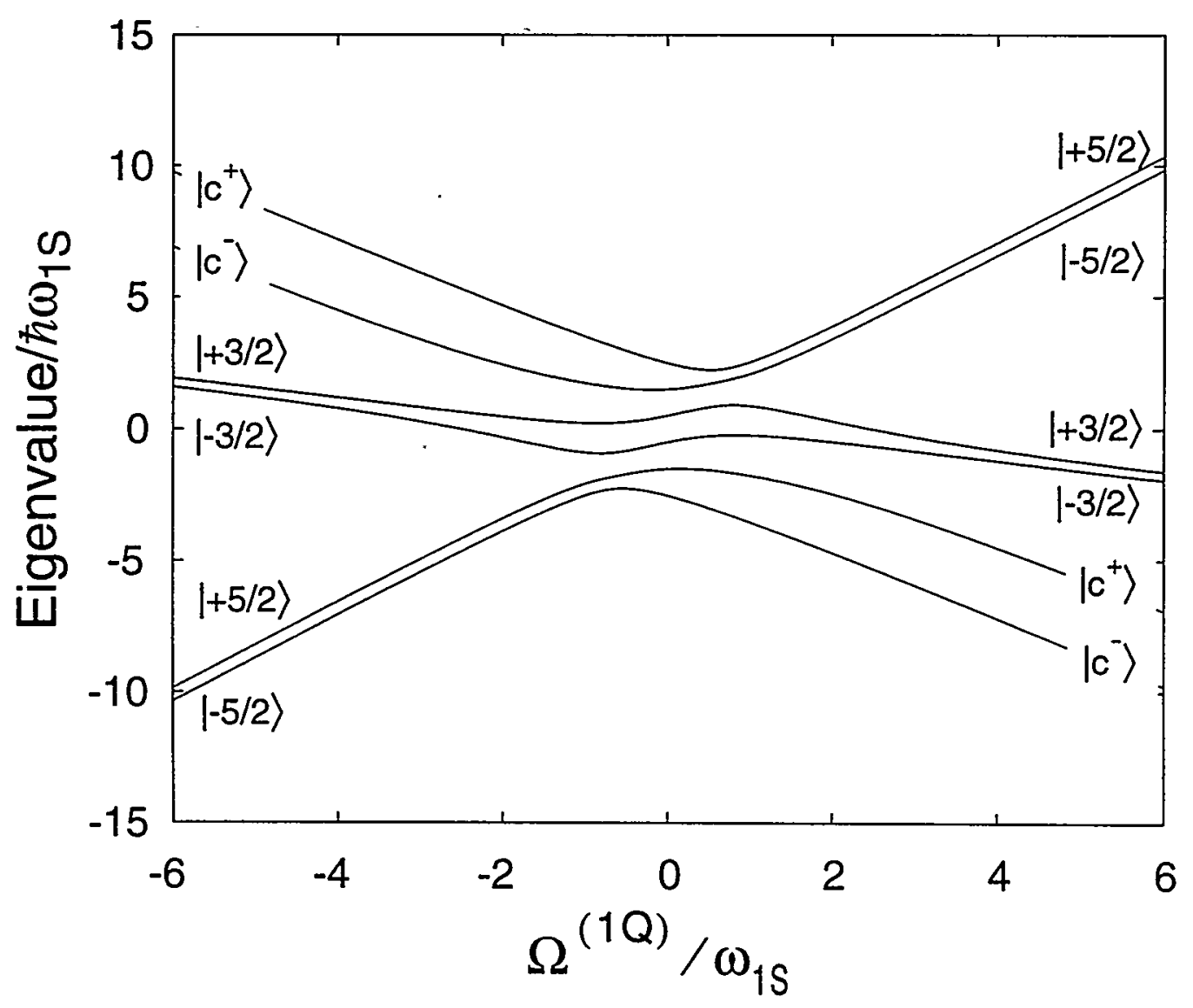

Figure 3.5 - Eigenvalues of Equation (3.1) where $S=5 / 2, \Delta \omega=0.1 \omega_{1 S}$, and $\overline{\mathcal{H}}_{\mathrm{Q}}$ is approximated by $\tilde{\mathscr{H}}_{Q}^{(1)}$. 
where $\omega_{\mathrm{r}}$ is the spinning speed. While the definition of the adiabaticity parameter can be refined to explicitly include individual crystallite orientations, ${ }^{11,122,126}$ Equation (3.8) is adequate for a qualitative description of the observed experimental behavior. For nuclei in which $\omega_{\mathrm{Q}} \gg \omega_{1 \mathrm{~S}}$, three regimes of spin-locking behavior have been defined based on the rate at which $\Omega^{(1 Q)}$ changes sign. In the adiabatic-passage regime $(\alpha \ll 1)$, the sign change is sufficiently slow that populations are transferred from their original eigenstate to the eigenstate that is derived by continuity. For the spin-5/2 case, this implies that the populations will oscillate between $\left|c^{ \pm}\right\rangle$and $| \pm 5 / 2\rangle$ as depicted in Figure 3.7a. In the sudden passage regime $(\alpha \ll 1)$, the sign change of the first-order quadrupolar Hamiltonian occurs too quickly for the populations to follow, and the populations remain in their original eigenstates. This is depicted schematically in Figure 3.7b. In both of these regimes, efficient spin-locking of the central transition is possible (although, as we will show below, there are some additional considerations). In the intermediate regime ( $\alpha \approx 1$ theoretically, $\alpha \approx 0.4$ experimentally ${ }^{120}$ ) the eigenstates are poorly defined, and spin locking is very inefficient.

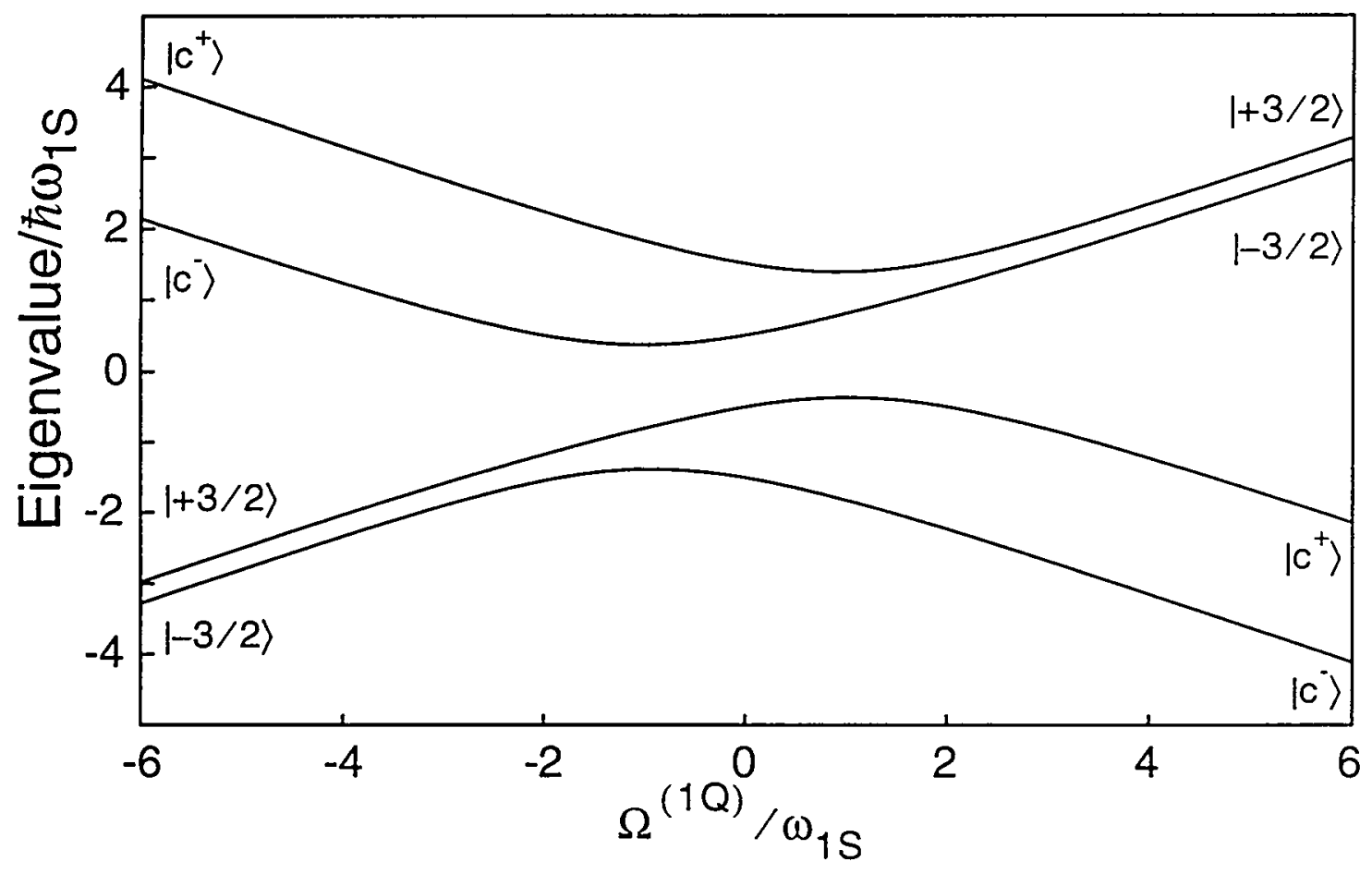

Figure 3.6 - Eigenvalues of Equation (3.1) where $S=3 / 2, \Delta \omega=0.1 \omega_{1 S}$, and $\overline{\mathcal{H}}_{\mathrm{Q}}$ is approximated by $\tilde{\mathcal{H}}_{Q}^{(1)}$ 

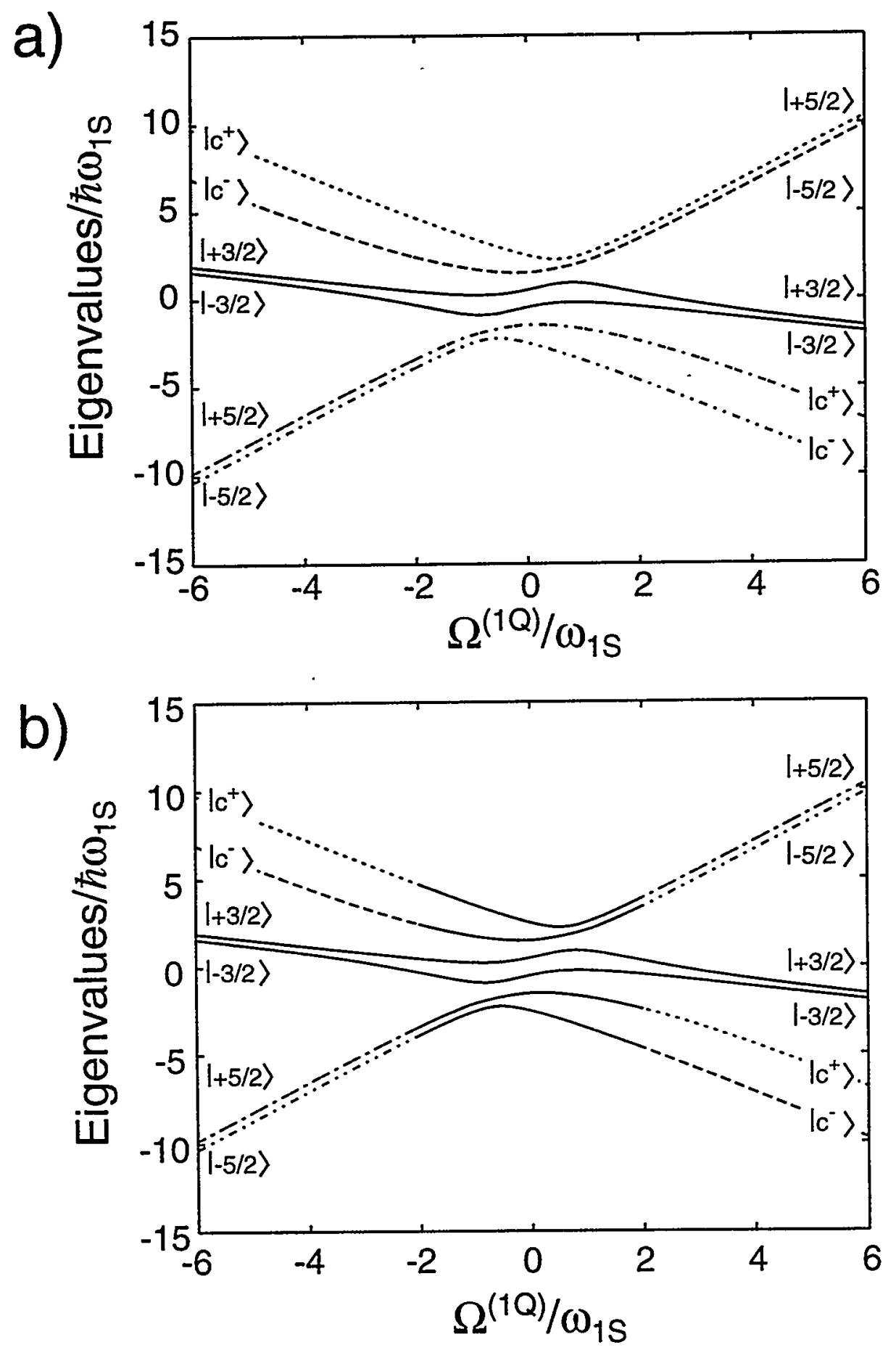

Figure 3.7 - Diagram of population transfers in different regimes. The patterned lines correspond to populations before and after a single zero crossing. (a) Adiabatic case. Populations are transferred to the eigenstates to which they are connected by continuity. (b) Sudden case. Populations remain in original eigenstates. 
This $\alpha$-dependent behavior has important implications for many experiments. In systems with vastly different $C_{q c c}$ 's, an if field of a given strength may not lock all sites. Although this is a disadvantage for purposes of quantitation, researchers have exploited this property and used spin locks as filters for spectral editing. ${ }^{127}$

With quadrupolar frequencies of the magnitude of those listed in Table 3.2 and with $\omega_{1 S}$ restricted to relatively low values due to the large coil size ${ }^{128}$ used in some of the experiments (see Chapter 4), spin locking in our cross-polarization experiments is limited to the sudden regime even at slow spinning speeds. In all simulations and measurements presented in Chapters 3, 4, and 5, $\alpha$ is less than 0.02 .

\subsection{Spin Locking in the Sudden Regime}

To analyze the detailed behavior of the aluminum spin-lock efficiency at slow spinning speeds and low-rf fields, experiments were performed on a home-built spectrometer incorporating a Tecmag acquisition system operating at a proton Larmor frequency of $301.2 \mathrm{MHz}$, which corresponds to ${ }^{27} \mathrm{Al}$ Larmor frequency of $78.5 \mathrm{MHz}$.

A Chemagnetics MAS probe with a $4 \mathrm{~mm}$ pencil rotor was used, and spinning speeds were regulated to within $\pm 5 \mathrm{~Hz}$ by a home-built spinning speed controller. ${ }^{129}$ For each of the one-dimensional experiments 64 scans were summed with a recycle time of 5 s. The MAS spinning speed was $4000 \mathrm{~Hz}$, and the selective $90^{\circ}$ pulse length on the aluminum central transition was $17 \mu \mathrm{s}$, which corresponds to an rf-field strength of $\omega_{1 \mathrm{~S}} /(2 \pi)=4900 \mathrm{~Hz}$.

Spectra using eight different spin-lock times $\left(\tau_{\mathrm{SL}}=10 \mu \mathrm{s}, 1 \mathrm{~ms}, 5 \mathrm{~ms}, 10 \mathrm{~ms}, 20\right.$ $\mathrm{ms}, 50 \mathrm{~ms}, 100 \mathrm{~ms}$, and $200 \mathrm{~ms}$ ) were recorded for each of twenty-eight different rf-field strengths in the range from $\omega_{1 \mathrm{~s}} /(2 \pi)=440 \mathrm{~Hz}$ to $\omega_{1 s^{\prime}} /(2 \pi)=4400 \mathrm{~Hz}$. This corresponds to a range of the adiabaticity parameter from $\alpha=5 \times 10^{-5}$ to $\alpha=5 \times 10^{-3}$. The integral of the central transition of the Fourier-transformed spectrum was used as a measure of the spin-lock efficiency for the corresponding if field strength. Figure 3.8a shows the spin-lock efficiency for spin-lock times $\left(\tau_{\mathrm{SL}}\right)$ of $10 \mu \mathrm{s}, 1 \mathrm{~ms}$, and $10 \mathrm{~ms}$. As expected, the intensity at $\tau_{\mathrm{SL}}=10 \mu \mathrm{s}$ is fairly constant and does not depend on the field strength. At longer spin-lock times, there is a very distinct dependence of the signal intensity on the rf-field strength. In addition to rotating-frame relaxation, there are dips at 

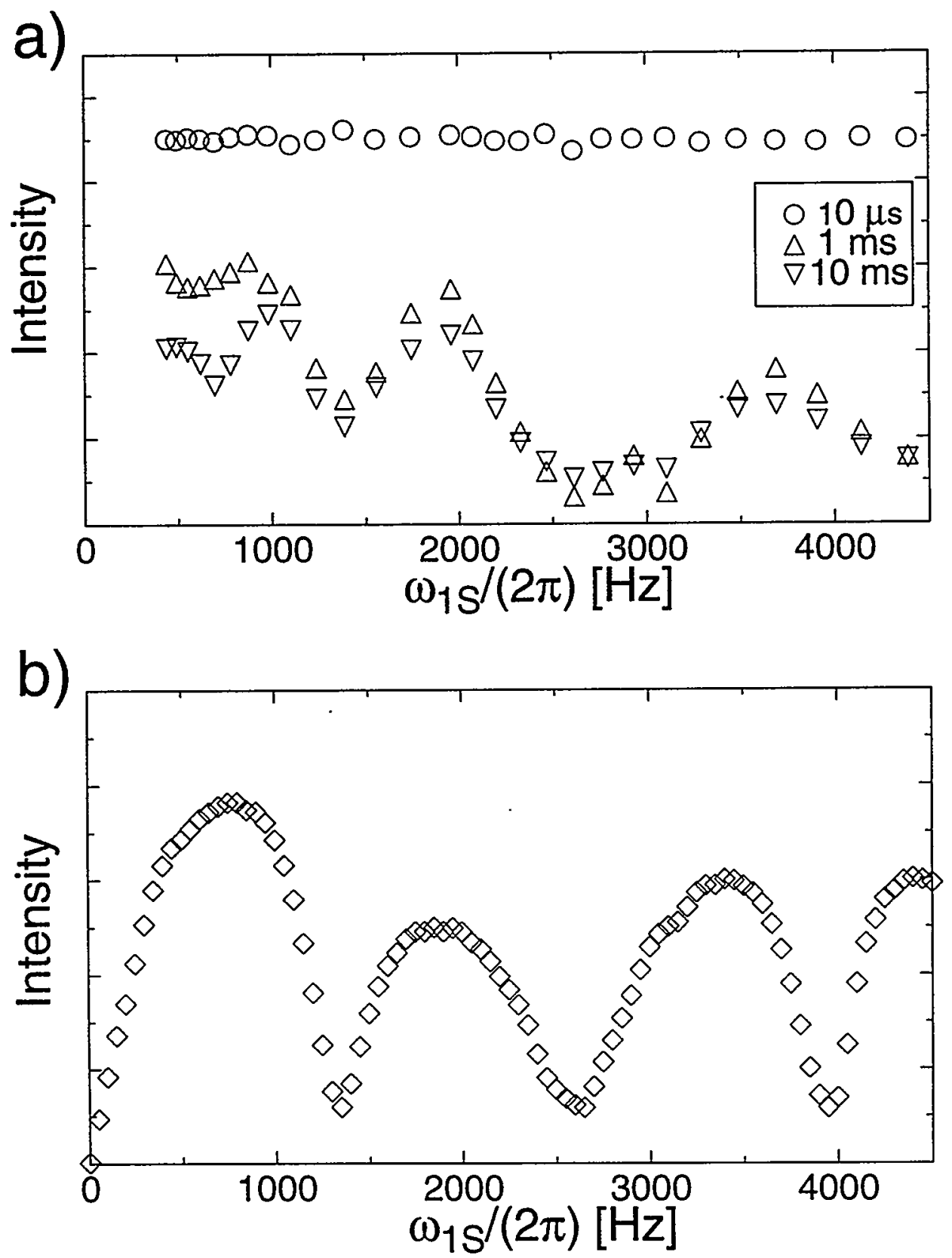

1

Figure 3.8 - Spin-lock efficiency for ${ }^{27} \mathrm{Al}$ in albite during magic-angle spinning $\left(\omega_{\mathrm{r}} /(2 \pi)=4000 \mathrm{~Hz}\right)$ as a function of the if field strength. The ${ }^{27} \mathrm{Al}$ Larmor frequency is $78.5 \mathrm{MHz}$. (a) Integrated intensity of the aluminum central transition at a function of the rf field strength for experiments with spin-lock times $\left(\tau_{\mathrm{SL}}\right)$ of $10 \mu \mathrm{s}[0], 1 \mathrm{~ms}[\Delta]$, and 10 ms $[\nabla]$. (b) Simulations of the "equilibrium" spin-lock efficiency for a spin-5/2 nucleus including both first- and second-order quadrupolar interactions and using the parameters 
spin-lock field strengths approximately equal to one-third, two-thirds, and one times the rotor frequency. The measured intensities for spin-lock times of $1 \mathrm{~ms}$ and $10 \mathrm{~ms}$ are very similar which indicates that the decay due to the time dependence of the quadrupolar interaction is very fast and occurs during the first few rotor cycles. The main differences between the spin-lock times of $1 \mathrm{~ms}$ and $10 \mathrm{~ms}$ occur at very low rf-field strengths and are most likely due to off-resonance effects.

The distinct dips in intensity found experimentally are clearly reflected in the numerical simulations shown in Figure $3.8 \mathrm{~b}$. These numerical simulations were performed using the NMR simulation package GAMMA. ${ }^{130}$ To solve the Liouville-vonNeumann equation for the time-dependent Hamiltonian, a small-step numerical integration with a time increment of $50 \mathrm{~ns}$ was performed. The simulations included the second-order quadrupolar Hamiltonian (Equation (3.4)) but omitted all relaxation effects. The Hilbert space was limited to a one-spin system; therefore, all scalar and dipolar couplings to other spins were neglected. The chemical-shielding tensor and chemicalshift offsets were also neglected in the simulations. A spinning speed of $4000 \mathrm{~Hz}$ and a ${ }^{27} \mathrm{Al}$ Larmor frequency of $78.5 \mathrm{MHz}$ were used. Three hundred different crystallite orientations were averaged using the method of Cheng et al. ${ }^{38}$ (see Section 1.3) to approximate all crystallite orientations present in a powder sample. The simulated timedomain data (intensity as a function of the spin-lock time) show a very rapid decay within the first $1 \mathrm{~ms}$ after which the intensity stabilizes and does not decay further due to the omission of relaxation effects from the numerical simulation. This equilibrium value is plotted as a function of the rf-field strength in Figure 3.8b. The simulations show the same characteristic decays of the spin-lock efficiency for rf-field strengths equal to one-third, two-thirds, and one times the rotor frequency as found in the experiment. At least some of the differences between the measurements and the simulations are due to the limitation of the simulation to a one-spin system. A similar level of agreement between experiment and simulation was found for other spinning speeds (data not shown).

A full theoretical analysis of the spin-lock behavior of quadrupolar nuclei under MAS is complicated by the fact that the Hamiltionian consists of a small time-independent term and a large time-dependent term. Consequently, perturbative approaches are not applicable, and analytical expressions for the interference process cannot be easily 
derived. Numerical simulations were, therefore, performed to determine whether the interference effects between the mechanical sample rotation and the spin-lock field strength are due to the first-order or to the second-order terms of the quadrupolar Hamiltonian. Simulations of the spin-lock efficiency for ${ }^{23} \mathrm{Na}$ (spin-3/2) and ${ }^{27} \mathrm{Al}$ (spin$5 / 2$ ) in low albite both with and without the second-order quadrupolar interaction were performed and are plotted as a function of the rf-field strength in Figure 3.9. The MAS frequency was set to $\omega_{\mathrm{r}} /(2 \pi)=2400 \mathrm{~Hz}$ which was the frequency used in the crosspolarization experiments of Chapter 4 , and 300 different crystallite orientations were averaged. The rf-field strength was varied between $\omega_{1 \mathrm{~s}} /(2 \pi)=0 \mathrm{~Hz}$ and $\omega_{1 S^{\prime}} /(2 \pi)=6800 \mathrm{~Hz}$ for both the ${ }^{23} \mathrm{Na}$ and ${ }^{27} \mathrm{Al}$ nuclei. These parameters result in values of $\alpha<0.019$ for ${ }^{27} \mathrm{Al}$ and $\alpha<0.015$ for ${ }^{23} \mathrm{Na}$, both well within the sudden passage regime. The Larmor frequencies were $130.31 \mathrm{MHz}$ for ${ }^{27} \mathrm{Al}$ and $132.28 \mathrm{MHz}$ for ${ }^{23} \mathrm{Na}$. The time increment for the numerical integration was $80 \mathrm{~ns}$. There are clear differences in the positions of the resonance dips between the spin-3/2 and the spin-5/2 simulations, but they follow a general rule. The spin-lock efficiency for simulations without the secondorder quadrupolar interaction (denoted by $\square$ in both parts of Figure 3.9) shows strong dips for the condition

$$
\frac{\omega_{1 S}}{\omega_{r}}=\frac{2 N}{S+1 / 2}
$$

where $\mathrm{N}$ is a positive integer and $\mathrm{S}$ is the spin-quantum number of the spin-locked quadrupolar nucleus. At these dips, the efficiency decays to approximately $50 \%$ of the non-resonance value. The number of dips predicted by considering only the first-order quadrupolar interaction is, however, insufficient to characterize the experimentally observed spin-lock efficiency.

In the simulations which include the both the first- and second-order quadrupolar interactions (denoted by $\diamond$ in Figures 1 and 2), the spin-lock efficiency decreases strongly when

$$
\frac{\omega_{1 S}}{\omega_{r}}=\frac{N}{S+1 / 2}
$$



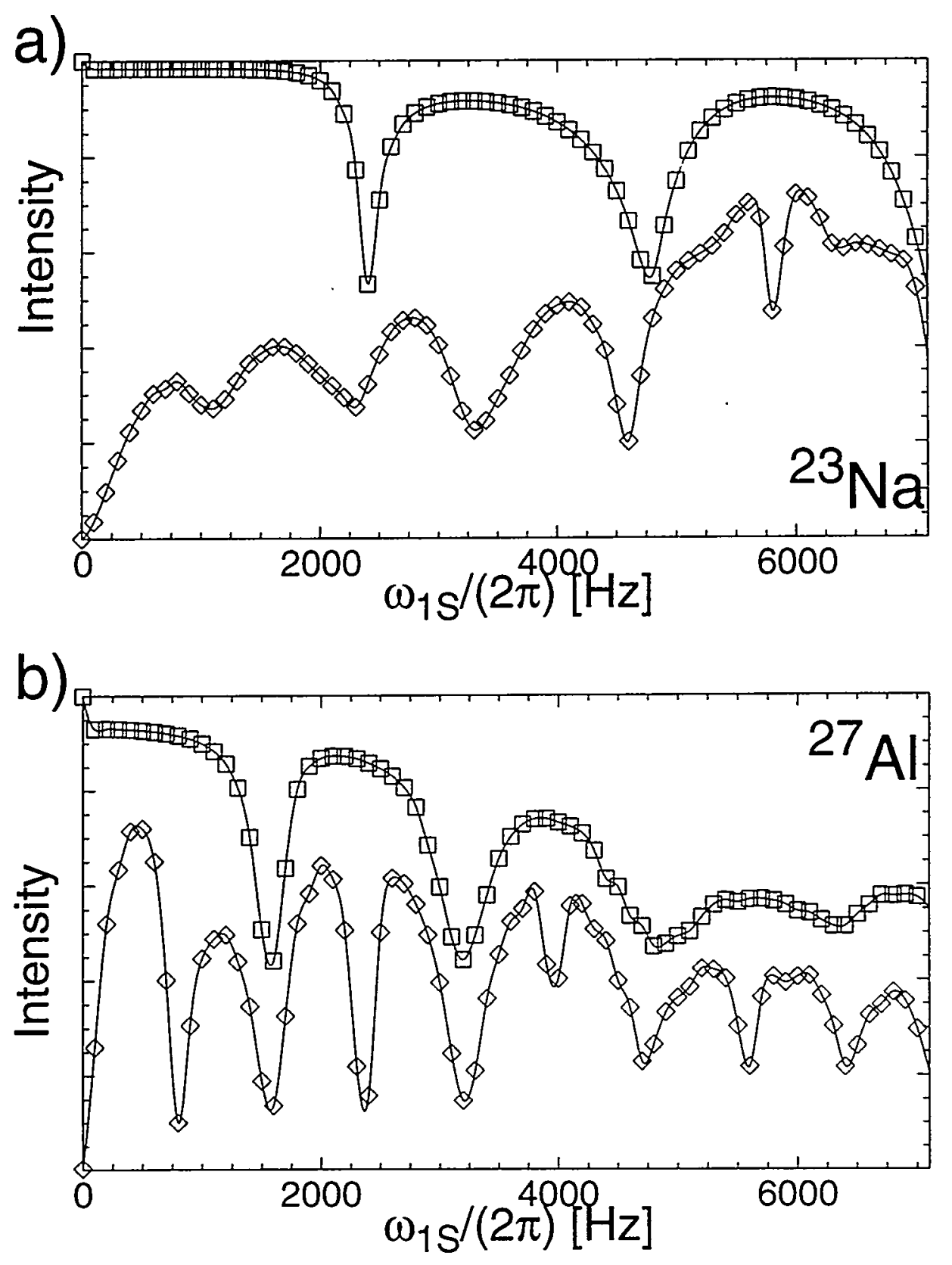

Figure 3.9 - (a) Simulations of the spin-lock efficiency for ${ }^{23} \mathrm{Na}(\mathrm{S}=3 / 2)$ at an MAS frequency of $\omega_{r} /(2 \pi)=2400 \mathrm{~Hz}$ both with [O] and without [ $\square$ ] the second-order quadrupolar interaction. (b) Simulations of the spin-lock efficiency for ${ }^{27} \mathrm{Al}(\mathrm{S}=5 / 2)$ at an MAS frequency of $\omega_{\mathrm{r}} /(2 \pi)=2400 \mathrm{~Hz}$ both with [ $\diamond$ ] and without [ $\square$ ] the secondorder quadrupolar interaction. Solid lines are guides to the eye. For all simulations the quadrupolar parameters of low albite were used. ${ }^{113}$ 
This leads to twice as many dips in the rf field dependence of the spin-lock efficiency as compared to the simulations without the second-order quadrupolar interaction and agrees with our experiments.

With increasing $\omega_{\mathrm{Q}}$, the overall efficiency of the spin lock decreases due to the large second-order quadrupolar Hamiltonian. This is the reason for the generally poorer spin-lock efficiency for the ${ }^{23} \mathrm{Na}$ in Figure $3.9 \mathrm{a}\left(\omega_{\mathrm{Q}}=8.11 \times 10^{6} \mathrm{rad} / \mathrm{s}\right)$ compared to the ${ }^{27} \mathrm{Al}$ in Figure $3.9 \mathrm{~b}\left(\omega_{\mathrm{Q}}=3.10 \times 10^{6} \mathrm{rad} / \mathrm{s}\right)$ for the simulations which include both firstand second-order terms. In making such comparisons, it is important to consider the values of $\omega_{\mathrm{Q}}$ rather than the more commonly tabulated $\mathrm{C}_{\mathrm{qcc}}$ values (see Equation (3.3)) since the $[2 S(2 S-1)]^{-1}$ scaling factor differs significantly for different values of $S$. In the case of low albite, for instance, the $\mathrm{C}_{\mathrm{qcc}}$ for ${ }^{23} \mathrm{Na}(\mathrm{S}=3 / 2)$ is less than the $\mathrm{C}_{\mathrm{qcc}}$ for ${ }^{27} \mathrm{Al}$ $(\mathrm{S}=5 / 2)$, but the value of $\omega_{\mathrm{Q}}$ for ${ }^{23} \mathrm{Na}$ is greater than the value of $\omega_{\mathrm{Q}}$ for ${ }^{27} \mathrm{Al}$. When

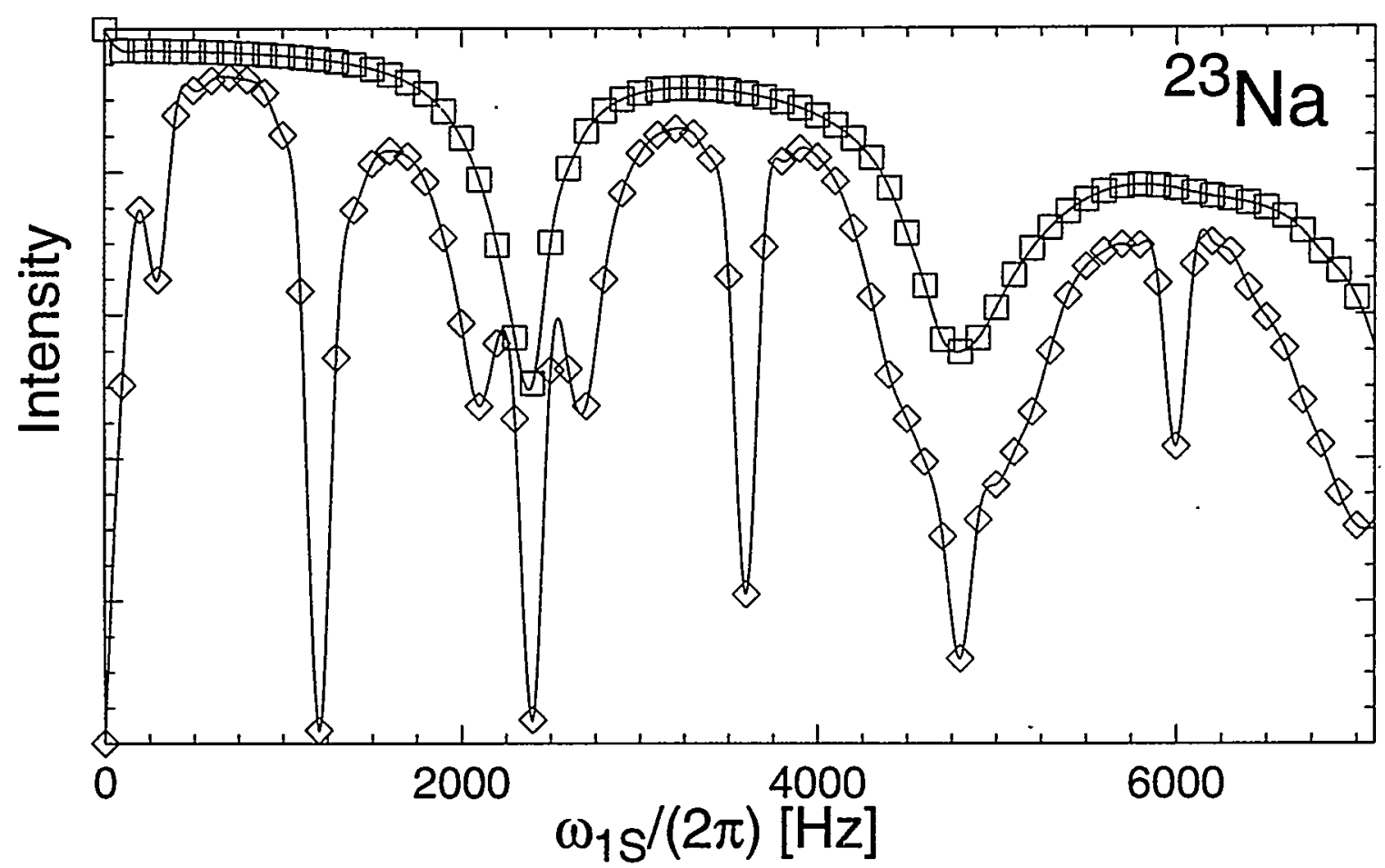

Figure 3.10 - Simulations of the spin-lock efficiency for ${ }^{23} \mathrm{Na}(\mathrm{S}=3 / 2)$ at an MAS frequency of $\omega_{\mathrm{r}} /(2 \pi)=2400 \mathrm{~Hz}$ and with $\omega_{\mathrm{Q}}=3.10 \times 10^{6} \mathrm{rad} / \mathrm{s}\left(\mathrm{C}_{\mathrm{qcc}}=0.99 \mathrm{MHz}\right)$ and $\eta=0.63$. Values are plotted both with $[\Delta]$ and without $[\square]$ the second-order quadrupolar interaction, and solid lines are guides to the eye. Note that with a smaller $\omega_{\mathrm{Q}}$, the dips are much sharper than in Figure 3.9a. 
smaller values of $\omega_{\mathrm{Q}}$ are used in the simulations for ${ }^{23} \mathrm{Na}$ (see Figure 3.10), the resonances at low rf-fields become sharper than in Figure 3.9a, and the spin-lock efficiency at points between the resonances increases.

The simulations shown in Figures 3.8b, 3.9 and 3.10 were performed for the case of on-resonance irradiation. When rf offsets of several hundred Hertz were incorporated into the simulations that only included the first-order contribution to the quadrupolar Hamiltonian (see Figure 3.11), the spin-locking behavior became qualitatively similar to the second-order case, and Equation 3.10 was obeyed. This is not surprising since both second-order shifts and if offsets can be expressed as linear combinations of fictitious spin-1/2 $\mathrm{S}_{\mathrm{z}}^{(\mathrm{a}, \mathrm{b})}$ operators ${ }^{4}$ connecting the $+\mathrm{m}$ and $-\mathrm{m}$ states. ${ }^{120}$

Clearly, both first-order and second-order terms of the quadrupolar Hamiltionian influence the spin-locking efficiency. However, it is important to consider the contributions of individual crystallite orientations to the powder average to determine whether the decreases in spin-lock efficiency are a property of the single-crystallite Hamiltonian itself or whether they are due to interference effects between different crystallite orientations. This has been partially discussed in the literature ${ }^{123}$ and is examined here in more detail.

Figures $3.12,3.13$, and 3.14 show spin-lock time dependences for a spin-5/2 nucleus $\left({ }^{27} \mathrm{Al}\right.$ in low albite) with (f-j) and without (a-e) the second-order quadrupolar interaction for four different orientations (a-d and $f-i$ ) and for the powder average (e and $j$ ). For the simulations of Figure 3.12, the spinning speed was $2400 \mathrm{~Hz}$, and the if field strength was $800 \mathrm{~Hz}$; these values satisfy the resonance condition of Equation (3.10) but not that of Equation (3.9). This is reflected in the fact that the simulations which exclude the second-order quadrupolar interaction (Figure 3.12a-e) show a good spin lock with only small-amplitude oscillations. Consequently the powder average (Figure 3.12e) shows almost no decay. Inclusion of the second-order quadrupolar interaction has a large effect on the spin-lock behavior (Figure 3.12f-j). The spin-locked magnetization oscillates between the positive and negative $x$-axis at a frequency that depends strongly on the crystallite orientation. The interference between these different oscillation frequencies causes the fast decay observed in the powder (Figure 3.12j). 


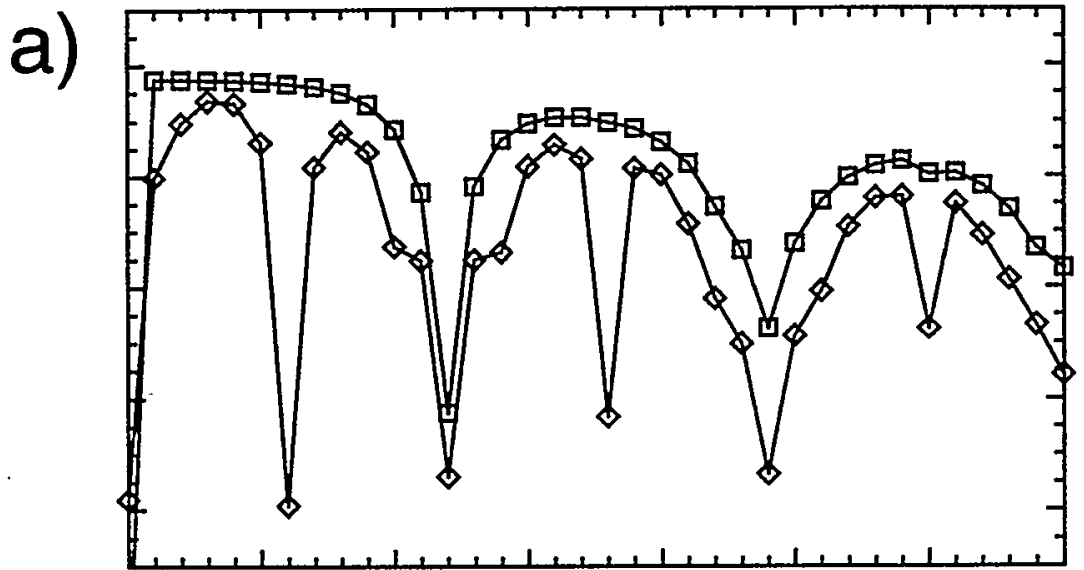

b)

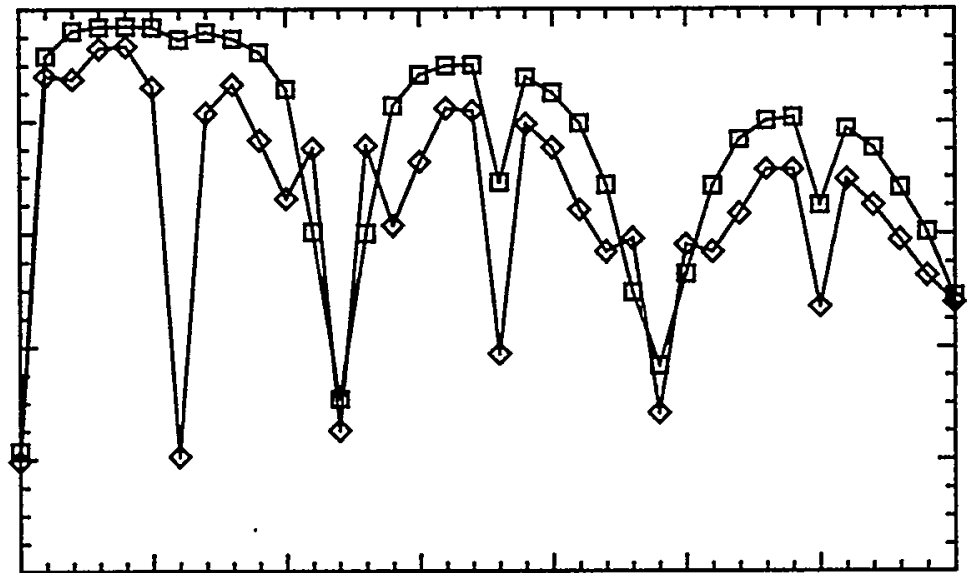

c)

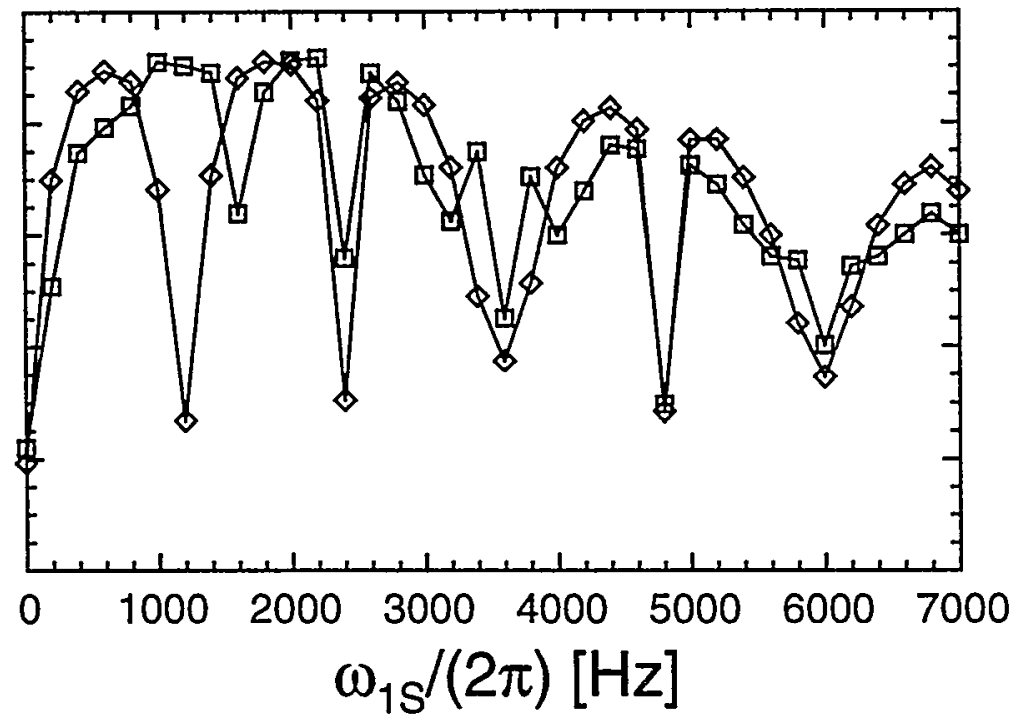

Figure 3.11 - Spin-lock efficiency as a function of resonance offset. The parameters of Figure 3.10 were used in these simulations. Values are plotted both with [0] and without [a] the second-order quadrupolar interaction, and solid lines are guides to the eye. (a) Offset of $10 \mathrm{~Hz}$. The behavior is qualitatively the same as for the on-resonance case. (b) Offset of $100 \mathrm{~Hz}$. Extra resonances begin to grow in making the first order simulations similar to the second order case. (c) Offset of $500 \mathrm{~Hz}$. Except for a slight distortion at low frequencies, the first and second-order cases are very similar now. 


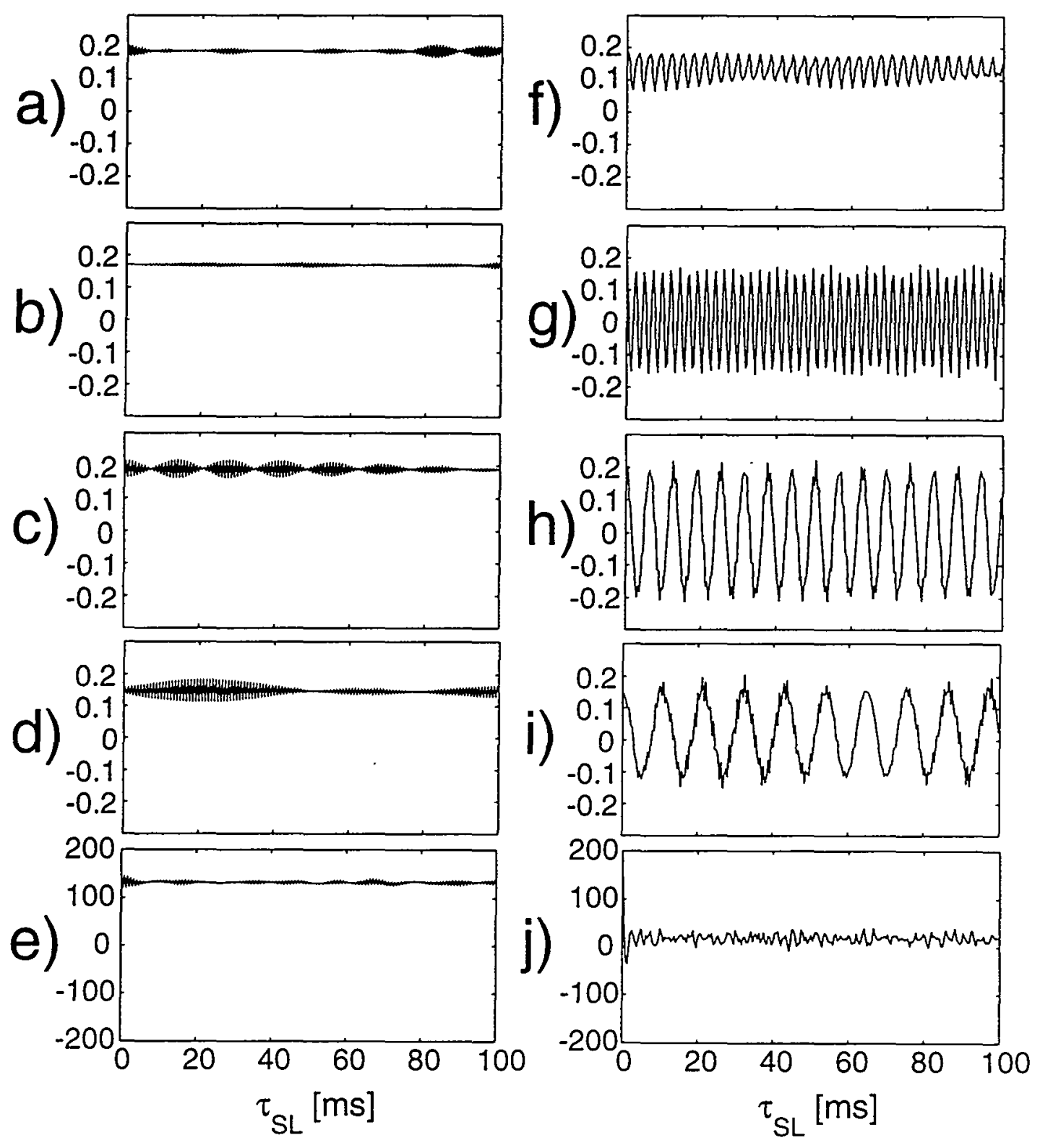

Figure 3.12 - Simulated spin-lock efficiency for ${ }^{27} \mathrm{Al}$ in low albite at an MAS frequency of $\omega_{\mathrm{r}} /(2 \pi)=2400 \mathrm{~Hz}$ and a spin-lock field strength of $\omega_{1 s^{\prime}}(2 \pi)=800 \mathrm{~Hz}$. The Euler angles $\left(\alpha^{Q}, \beta^{Q}, \gamma^{Q}\right)$ relate the principal-axis system of the quadrupolar interaction in a given crystallite to the reference frame of a rotor spinning at the magic angle. The simulations (a)-(e) were done without the second-order quadrupolar interaction while the simulations (f)-(j) include the second-order quadrupolar interaction. (a)-(d) and (f)-(i) show selected crystallite orientations with $\alpha^{\mathrm{Q}}=\gamma^{\mathrm{Q}}=0^{\circ}$ and $\beta^{\mathrm{Q}}=10^{\circ}$ ((a) and (f)), $\beta^{\mathrm{Q}}=30^{\circ}$ (b) and (g)), $\beta^{\mathrm{Q}}=50^{\circ}((\mathrm{c})$ and $(\mathrm{h})), \beta^{\mathrm{Q}}=70^{\circ}((\mathrm{d})$ and (i)). The simulations in (e) and (j) show the average over 1154 different crystallite orientations. For this choice of spinning speed and if field strength, only the resonance condition of Equation (3.10) is fulfilled. Therefore, the spin lock is very stable without the second-order quadrupolar interaction (e) but decays rapidly when the second-order quadrupolar interaction is included $(j)$. 

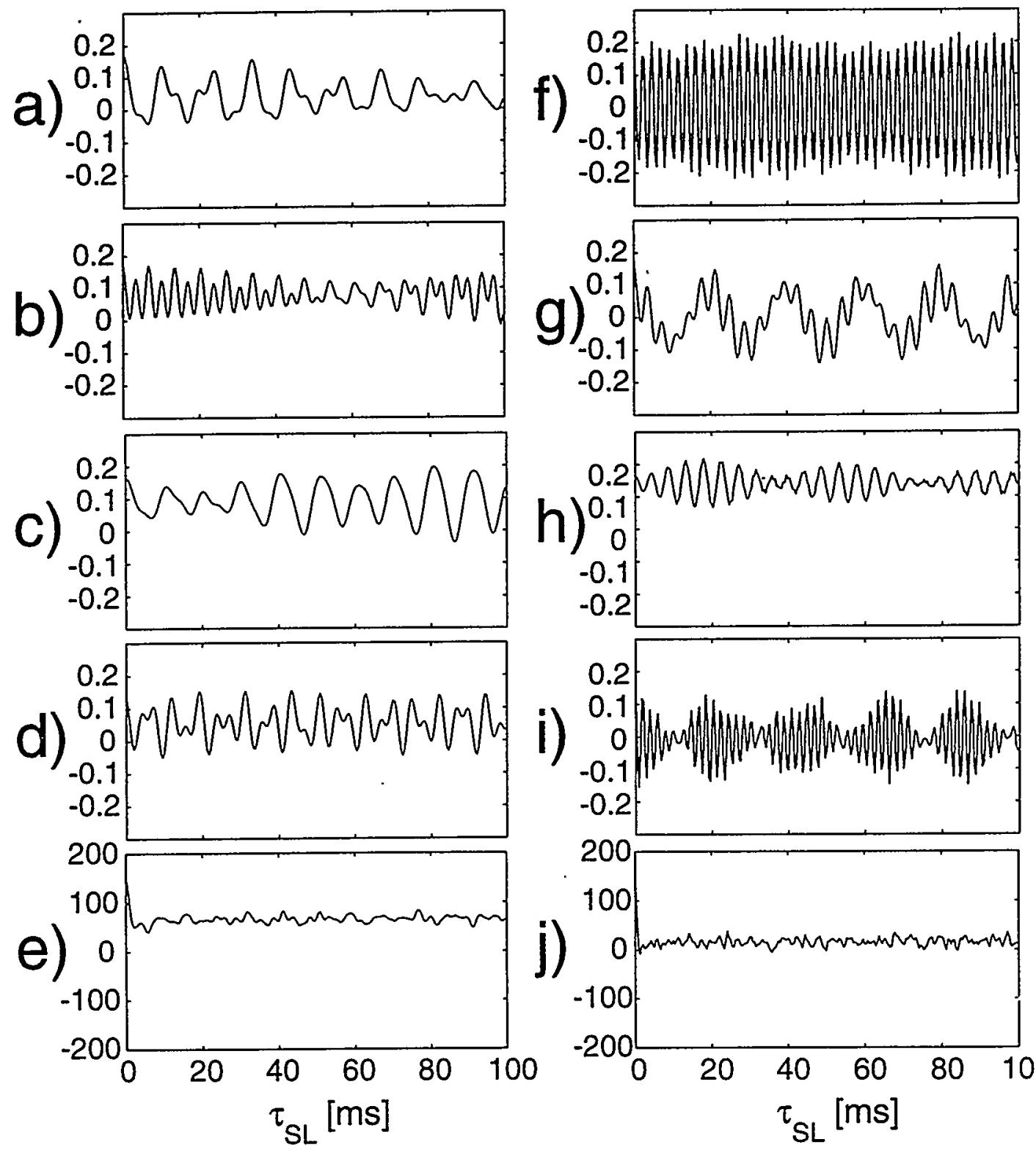

Figure 3.13 - Simulated spin-lock efficiency for ${ }^{27} \mathrm{Al}$ in low albite at an MAS frequency of $\omega_{\mathrm{r}} /(2 \pi)=2400 \mathrm{~Hz}$ and a spin-lock field strength of $\omega_{1 S^{\prime}} /(2 \pi)=1600 \mathrm{~Hz}$. The Euler angles $\left(\alpha^{\mathrm{Q}}, \beta^{\mathrm{Q}}, \gamma^{\mathrm{Q}}\right)$ relate the principal-axis system of the quadrupolar interaction in a given crystallite to the reference frame of a rotor spinning at the magic angle. The simulations (a)-(e) were done without the second-order quadrupolar interaction while the simulations (f)-(j) include the second-order quadrupolar interaction. (a)-(d) and (f)-(i) show selected crystallite orientations with $\alpha^{Q}=\gamma^{Q}=0^{\circ}$ and $\beta^{Q}=10^{\circ}$ ((a) and (f)), $\beta^{Q}=30^{\circ}$ ((b) and (g)), $\beta^{Q}=50^{\circ}((\mathrm{c})$ and $(\mathrm{h})), \beta^{\mathrm{Q}}=70^{\circ}(\mathrm{d})$ and (i)). The simulations in (e) and (j) show the average over 1154 different crystallite orientations. For this choice of spinning speed and rf field strength, the resonance conditions of Equation (3.9) and (3.10) are both fulfilled. Therefore, we see a fast decay of the spin-locked magnetization both with (j) and without (e) the second-order quadrupolar interaction. 

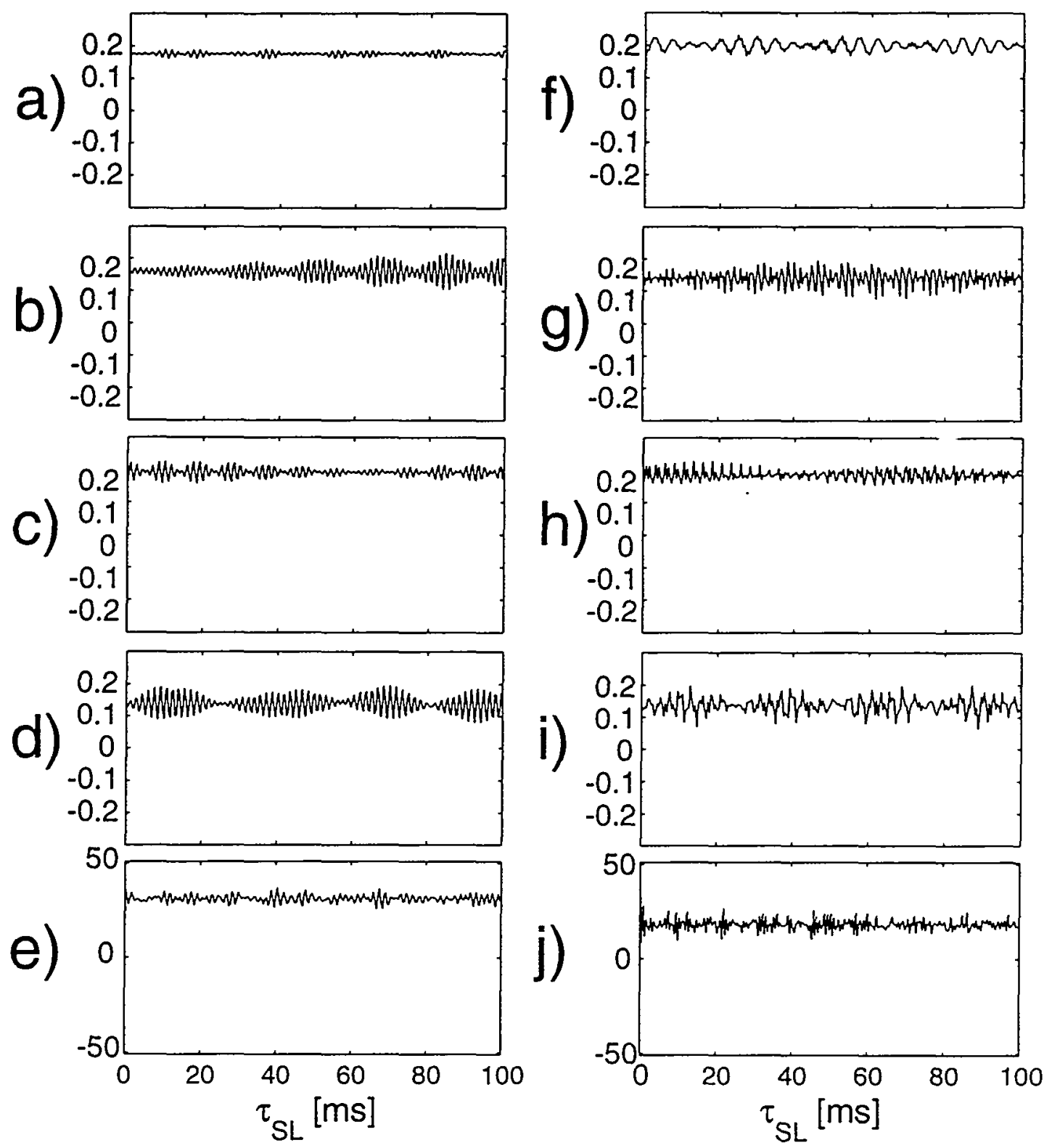

Figure 3.14 - Simulated spin-lock efficiency for ${ }^{27} \mathrm{Al}$ in low albite at an MAS frequency of $\omega_{r^{\prime}}(2 \pi)=2400 \mathrm{~Hz}$ and a spin-lock field strength of $\omega_{1 s^{\prime}} /(2 \pi)=1200 \mathrm{~Hz}$. The Euler angles $\left(\alpha^{\mathrm{Q}}, \beta^{\mathrm{Q}}, \gamma^{\mathrm{Q}}\right)$ relate the principal-axis system of the quadrupolar interaction in a given crystallite to the reference frame of a rotor spinning at the magic angle. The simulations (a)-(e) were done without the second-order quadrupolar interaction while the simulations (f)-(j) include the second-order quadrupolar interaction. (a)-(d) and (f)-(i) show selected crystallite orientations with $\alpha^{Q}=\gamma^{\mathrm{Q}}=0^{\circ}$ and $\beta^{\mathrm{Q}}=10^{\circ}\left((\mathrm{a})\right.$ and $(\mathrm{f})$ ), $\beta^{\mathrm{Q}}=30^{\circ}$ $((\mathrm{b})$ and $(\mathrm{g})), \beta^{\mathrm{Q}}=50^{\circ}((\mathrm{c})$ and $(\mathrm{h})), \beta^{\mathrm{Q}}=70^{\circ}((\mathrm{d})$ and $(\mathrm{i}))$. The simulations in (e) and (j) show the average over 1154 different crystallite orientations. For this choice of spinning speed and rf field strength, neither the resonance condition of Equation (3.9) nor that of Equation (3.10) is fulfilled. Therefore, the spin lock is stable both with (j) and without (e) the second-order quadrupolar interaction. 
For the simulations of Figure 3.13, the resonance conditions of Equations (3.9) and (3.10) are simultaneously satisfied by setting the spinning speed equal to $2400 \mathrm{~Hz}$ and the rf-field strength equal to $1600 \mathrm{~Hz}$. In Figure 3.13 there are strong oscillations for both the simulations without (Figure 3.13a-e) and with (Figure 3.13f-j) the second-order quadrupolar interaction. However, when the second-order quadrupolar interaction is omitted, the oscillations are only between the positive maximum and zero (Figure 3.13ad) leading to a reduced but non-vanishing value for the powder average (Figure 3.13e). Inclusion of the second order interaction results again in oscillation between the positive maximum and its ccrresponding negative value (Figure 3.13f-i). Consequently the powder average is almost zero (Figure 3.13j) due to the interference of magnetization from different crystallite orientations. Simulations for spin-lock fields in between resonance points (see Figure 3.14) show only small oscillations about non-zero values and thus good spin-lock efficiency. Similar simulations were calculated for spin-3/2 nuclei (data not shown) and show the same general behavior as the spin-5/2 simulations discussed above.

Note that the powder-averaged simulations of the spin lock at the resonance conditions (see, for instance, Figure 3.12j) show oscillations for short spin-lock times $\left(\tau_{\mathrm{SL}}<5 \mathrm{~ms}\right.$ ); such oscillations were also observed experimentally (data not shown). Fourier transformation of these reveals lineshapes that look qualitatively similar to rotary resonance spectra ${ }^{131}$ of spin-1/2 nuclei in which the chemical-shielding anisotropy is recoupled by setting the spin-lock field equal to one or two times the spinning frequency. Work is currently in progress to understand the nature of the lineshapes observed in the quadrupolar case and to determine whether the quadrupolar parameters can be extracted from such spectra.

The resonance conditions defined by Equation 3.10 have an important consequence for cross-polarization experiments involving half-integer quadrupolar spins. Before setting up the cross-polarization condition, it is necessary to experimentally optimize the spin-lock efficiency of the quadrupolar nucleus for a given sample (i.e. given $\omega_{Q}$ ) and spinning speed in order to avoid a severe loss of magnetization. 


\subsection{Direct measurement of the ${ }^{27} \mathrm{Al}$ and ${ }^{23} \mathrm{Na} \mathrm{T}_{1 \rho}$ 's in low albite}

In addition to losses due to inefficient spin locking, the magnetization of quadrupolar nuclei under spin-lock conditions is further influenced by rotating-frame spin-lattice relaxation. Unlike for the case of spin-1/2 nuclei, the rotating-frame behavior of a quadrupolar nucleus cannot in general be modeled by a single exponential. ${ }^{26,132,133}$ Consequently, no single relaxation time constant $\left(T_{1 \rho}\right)$ can be defined. In our experimental observations, rotating-frame relaxation of aluminum and sodium in albite consists of at least two exponentially decaying components. Figure 3.15 shows such a decay for ${ }^{27} \mathrm{Al}$ in albite for $\omega_{1 \mathrm{~S}} /(2 \pi) \approx 500 \mathrm{~Hz}$ and $\omega_{\mathrm{r}} /(2 \pi)=2400 \mathrm{~Hz}$ (the values used in the cross-polarization experiments of Chapter 4). The solid curve in Figure 3.15 corresponds to a least-squares fit of the data to a biexponential function of the form

$$
I(t)=A \cdot e^{-t / \tau_{f}}+B \cdot e^{-t / \tau_{s}}
$$

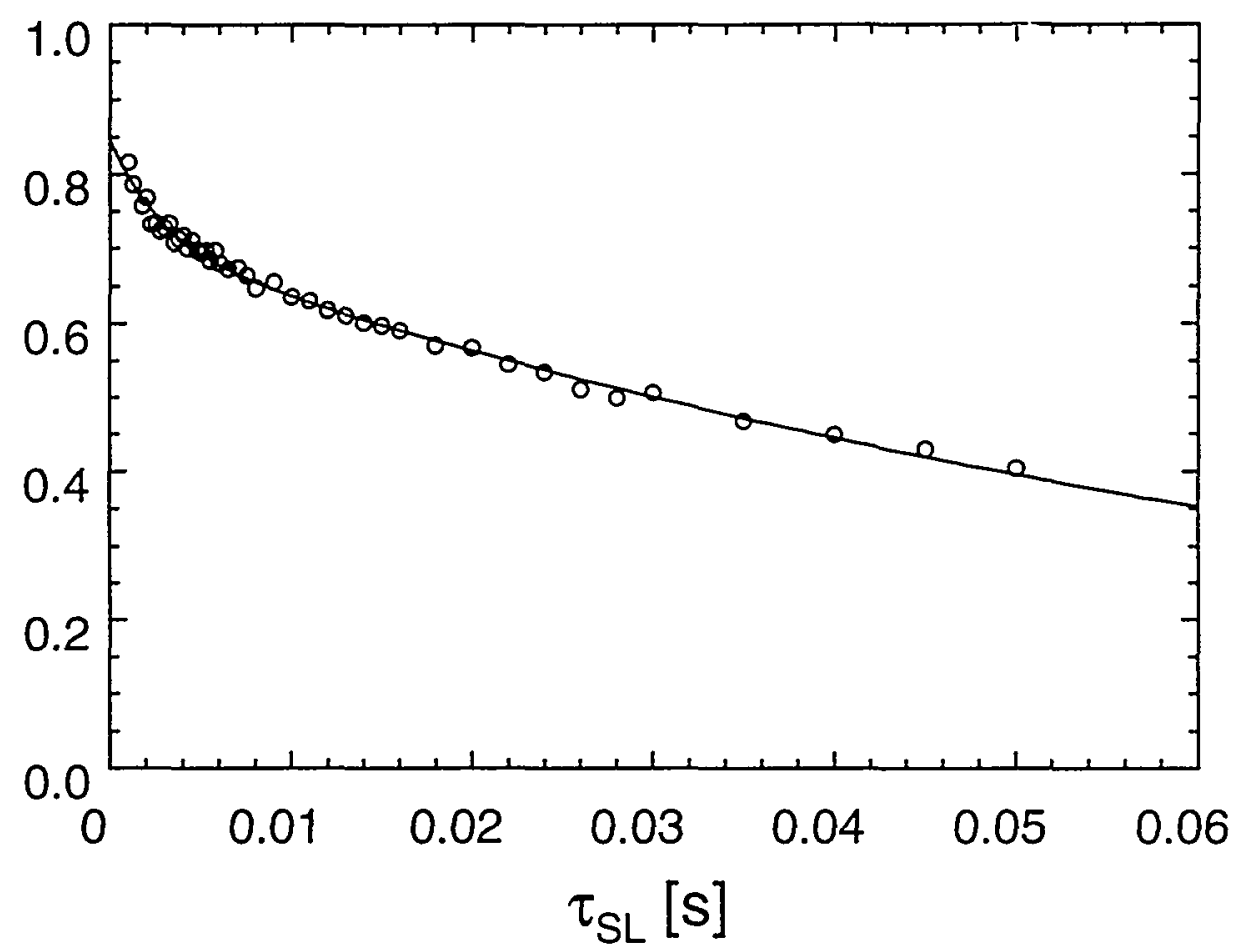

Figure 3.15 - Rotating-frame relaxation of ${ }^{27} \mathrm{Al}$ in albite at $\omega_{1 \mathrm{~S}} /(2 \pi) \approx 500 \mathrm{~Hz}$ and $\omega_{\mathrm{r}} /(2 \pi)=2400 \mathrm{~Hz}$. The solid line is a nonlinear least-squares fit of the experimental points to a biexponential function with time constants of 2.7 and $85 \mathrm{~ms}$. 
in which the fast-decaying component is characterized by a time constant $\tau_{\mathrm{f}}=2.7 \mathrm{~ms}$; the slowly-decaying component is characterized by a time constant $\tau_{\mathrm{s}}=85 \mathrm{~ms}$; and the scaling parameters have values of $A=0.13$ and $B=0.71$. The rotating frame relaxation of ${ }^{23} \mathrm{Na}$ in albite also requires more than one exponential for a good fit (see Figure 3.16). With typical cross-polarization contact times on the order of $\tau_{\mathrm{CP}}=10 \mathrm{~ms}$ to $\tau_{\mathrm{CP}}=50 \mathrm{~ms}$, the rotating-frame relaxation of the quadrupolar nuclei in low albite poses no problem for efficient cross polarization.

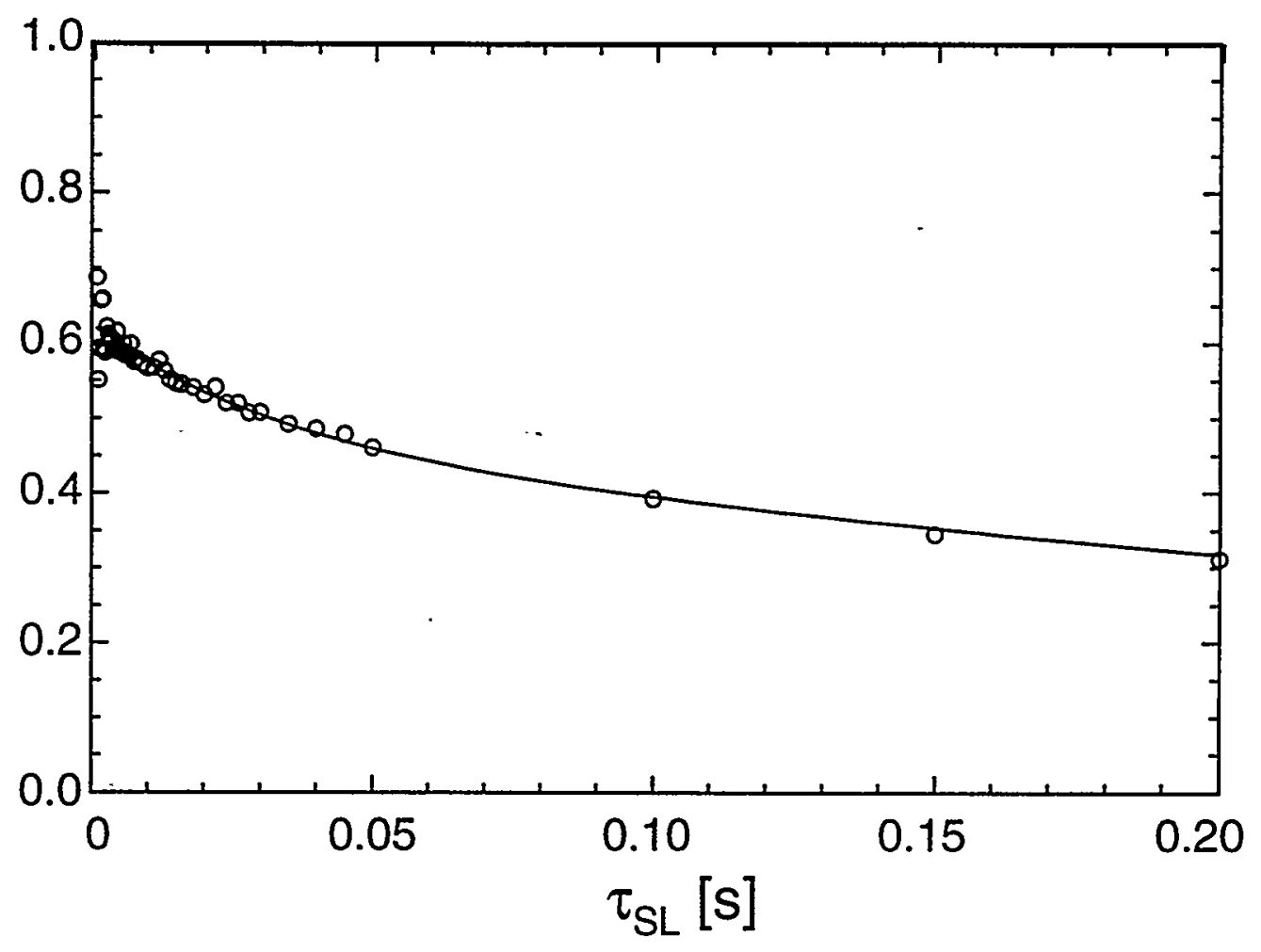

Figure 3.16 - Rotating-frame relaxation of ${ }^{23} \mathrm{Na}$ in albite at $\omega_{1 \mathrm{~s}} /(2 \pi) \approx 500 \mathrm{~Hz}$ and $\omega_{\mathrm{r}} /(2 \pi)=2400 \mathrm{~Hz}$. The solid line is a nonlinear least-squares fit of the experimental points to a biexponential function with time constants of 9.1 and $28 \mathrm{~ms}$. Note that even with a biexponential, the first points are not well fit. However, a mono-exponential fit fails completely. 


\section{Chapter 4: Cross Polarization of Quadrupolar Nuclei During MAS Using Low Radiofrequency Field Amplitudes}

Using the results of the spin-lock efficiency study of Chapter 3 , the ${ }^{27} \mathrm{Al}-$ to- $^{29} \mathrm{Si}$ cross-polarization dynamics in low albite were investigated. Although cross polarization was achieved from both ${ }^{27} \mathrm{Al}$ and ${ }^{23} \mathrm{Na}$, we chose to concentrate on ${ }^{27} \mathrm{Al}$ since the crosspolarized signals were more intense. As mentioned in Section 3.1, albite has three different silicon sites (see Table 3.1): two of which $\left(\mathrm{Q}^{4}(1 \mathrm{Al})\right.$ ) have one aluminum atom and three silicon atoms as nearest neighbors while the third $\left(\mathrm{Q}^{4}(2 \mathrm{Al})\right)$ has two aluminum atoms and two silicon atoms as nearest neighbors. Based on simple models one might expect that the NMR signal intensity corresponding to the silicon with two aluminum atoms as nearest neighbors would be twice as intense as those of the silicons with only one aluminum atom as a nearest neighbor. ${ }^{134}$ However, as a one-dimensional ${ }^{29} \mathrm{Si}$ NMR spectrum acquired using ${ }^{27} \mathrm{Al}-\mathrm{to}^{29} \mathrm{Si}$ cross polarization shows, this is not true in the case of low albite (Figure 4.1). The two silicon sites in albite with one aluminum nearest neighbor ( $\delta=-96.1 \mathrm{ppm}$ and $\delta=-103.9 \mathrm{ppm}$ ) have equal intensities while the site with two aluminum nearest neighbors $(\delta=-91.8 \mathrm{ppm})$ has a lower intensity. There is no significant difference in the line widths of the three lines: the full width at halfmaximum of a Lorentzian line fit was $\Delta \omega_{1 / 2} \approx 35 \mathrm{~Hz}$ for all three lines. To investigate this behavior in more detail, we have performed cross-polarization contact-time dependence measurements as well as rotating-frame relaxation time measurements for the three silicon sites.

\subsection{Previous Studies of Cross Polarization Involving Quadrupolar Nuclei}

Cross polarization to and from quadrupolar nuclei is becoming an increasingly popular technique. Although cross polarization under static conditions is easier to achieve and interpret, ${ }^{135}$ the ubiquity of MAS probes and the development of the MultipleQuantum Magic-Angle Spinning technique (see Chapter 6) has made an understanding of cross polarization to and from quadrupolar nuclei during MAS desirable. In this thesis, 
we will only be concerned with the case of cross polarization to and from the central transition of an odd-half-integer quadrupolar nucleus, although multiple-quantum cross poiarization is conceivable. ${ }^{136,137,138}$

The earliest CP/MAS experiments involving quadrupolar nuclei were largely empirical in nature. ${ }^{139,140,141}$ In 1992, A. J. Vega extended his quadrupolar spin-lock theory ${ }^{120}$ (see Section 3.2) to cross polarization ${ }^{121}$ and compared the effects of the adiabatic and sudden regimes. He showed that cross polarization occurs continuously in the sudden regime although only a subset of the nuclei participate. The reason for this is that populations that were initially in $a+3 / 2$ or $\pm 5 / 2$ state (see Figure $3.7 \mathrm{~b}$ ) are not interconverted to the central transition by sample rotation and, therefore, cannot participate in the cross polarization-process (although relaxation effects could, of course, alter the populations of different states). In the adiabatic regime, population interconversions do occur (see Figure 3.7a), permitting cross polarization from spin-1/2

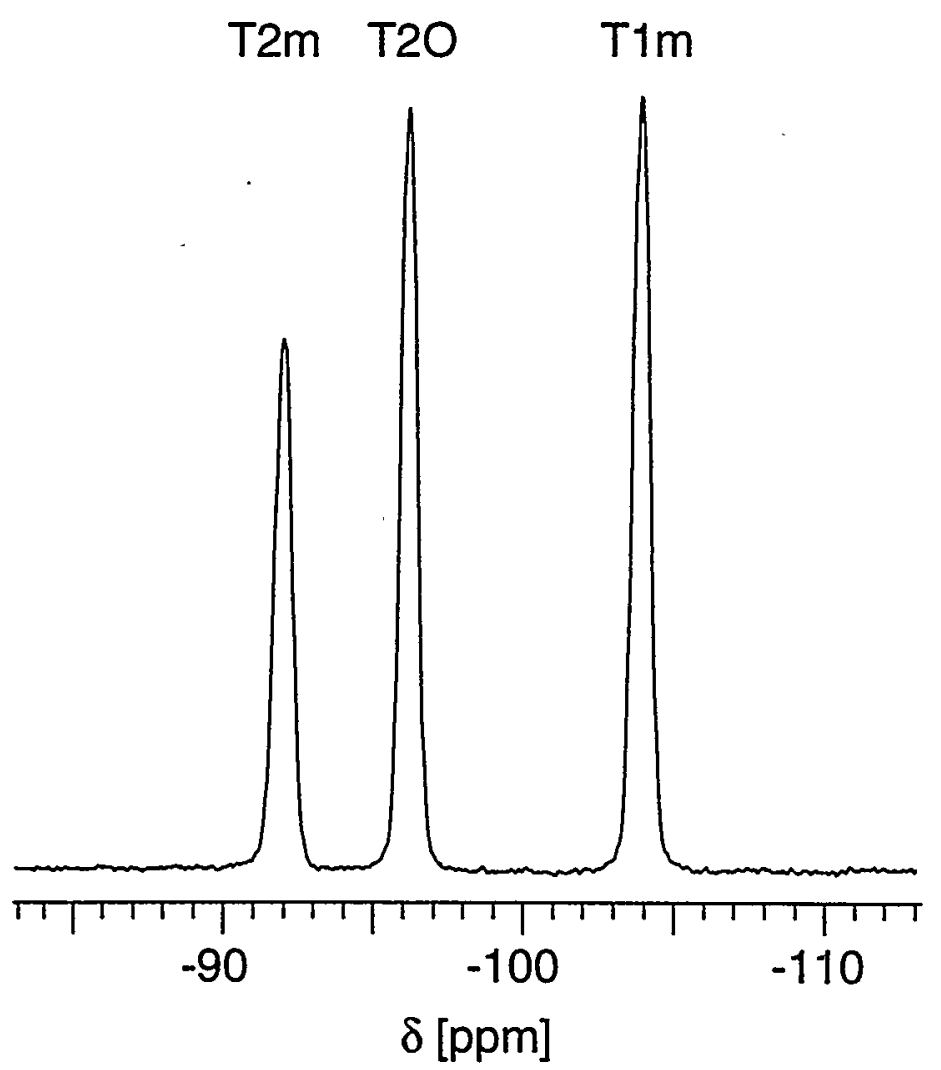

Figure 4.1 - One-dimensional ${ }^{29} \mathrm{Si}$ spectrum of low albite obtained with cross polarization from ${ }^{27} \mathrm{Al}$. The crystallographic sites are indicated at the three different peaks. The peak at $\delta=-91.8 \mathrm{ppm}$ shows the lowest intensity although this silicon site has two nearestneighbor aluminum atoms. The other two lines at $\delta=-96.1 \mathrm{ppm}$ and $\delta=-103.9 \mathrm{ppm}$ have roughly the same intensity. 
nuclei to a larger fraction of the quadrupolar spins. When cross polarizing from quadrupolar to spin-1/2 nuclei, however, the preparatory pulse will just excite populations that are initially in the central transition so these spins are the only ones which can participate. Since Vega's work, more applications of cross polarization from quadrupolar nuclei have appeared in the literature. ${ }^{134,142,143,144,145}$ Although some of these papers included studies of the cross-polarization dynamics, erroneous assumptions were made in a few instances. In particular, simplifications which were incompatible with measured values were used in models of cross-polarization dynamics. ${ }^{134}$

Due to the inefficiency of spin locking and cross polarizing quadrupolar nuclei under MAS conditions, angle-reorientation experiments are desirable. Such experiments take advantage of the fact that when the rotor axis is parallel to the $\mathrm{B}_{0}$ field, timedependent terms in the Hamiltonian vanish (see Section 1.4.1) which leads to cross polarization that is as efficient as in a static sample. Such experiments have been successfully performed, ${ }^{122,144}$ and their advantages and disadvantages as compared to the MAS experiment will be discussed below (see Section 4.5)

\subsection{Experimental Parameters}

All cross-polarization experiments were performed on a Chemagnetics CMX-500 spectrometer operating at a proton Larmor frequency of $500.1 \mathrm{MHz}$. The ${ }^{27} \mathrm{Al}$ frequency

was $130.31 \mathrm{MHz}$; the ${ }^{23} \mathrm{Na}$ frequency was $132.28 \mathrm{MHz}$; and the ${ }^{29} \mathrm{Si}$ frequency was 99.34 MHz.

Figure 4.2 shows the circuit diagram for the home-built double-resonance MAS probe used in these experiments. The probe incorporated a Chemagnetics "jumbo" spinning module with $14 \mathrm{~mm}$ outer diameter zirconia rotors. The volume of the rotors was approximately $2.8 \mathrm{~mL}$. The spinning speed was controlled by a home-built spinningspeed controller to an accuracy of $\pm 2 \mathrm{~Hz}$. An MAS speed of $2400 \mathrm{~Hz}$ was used for all CP experiments and silicon $T_{1 \rho}$ measurements described in this section. Typically, a 10-17 $\mu \mathrm{s}$ pulse was used for selective excitation of the central transition of aluminum. The cross polarization was optimized experimentally based on the spin-lock efficiency for the central transition of the quadrupolar nucleus as a function of the rf-field strength. At $\omega_{\mathrm{r}} /(2 \pi)=2400 \mathrm{~Hz}$, the best spin-lock level for the ${ }^{27} \mathrm{Al}$ resonance was found to be 


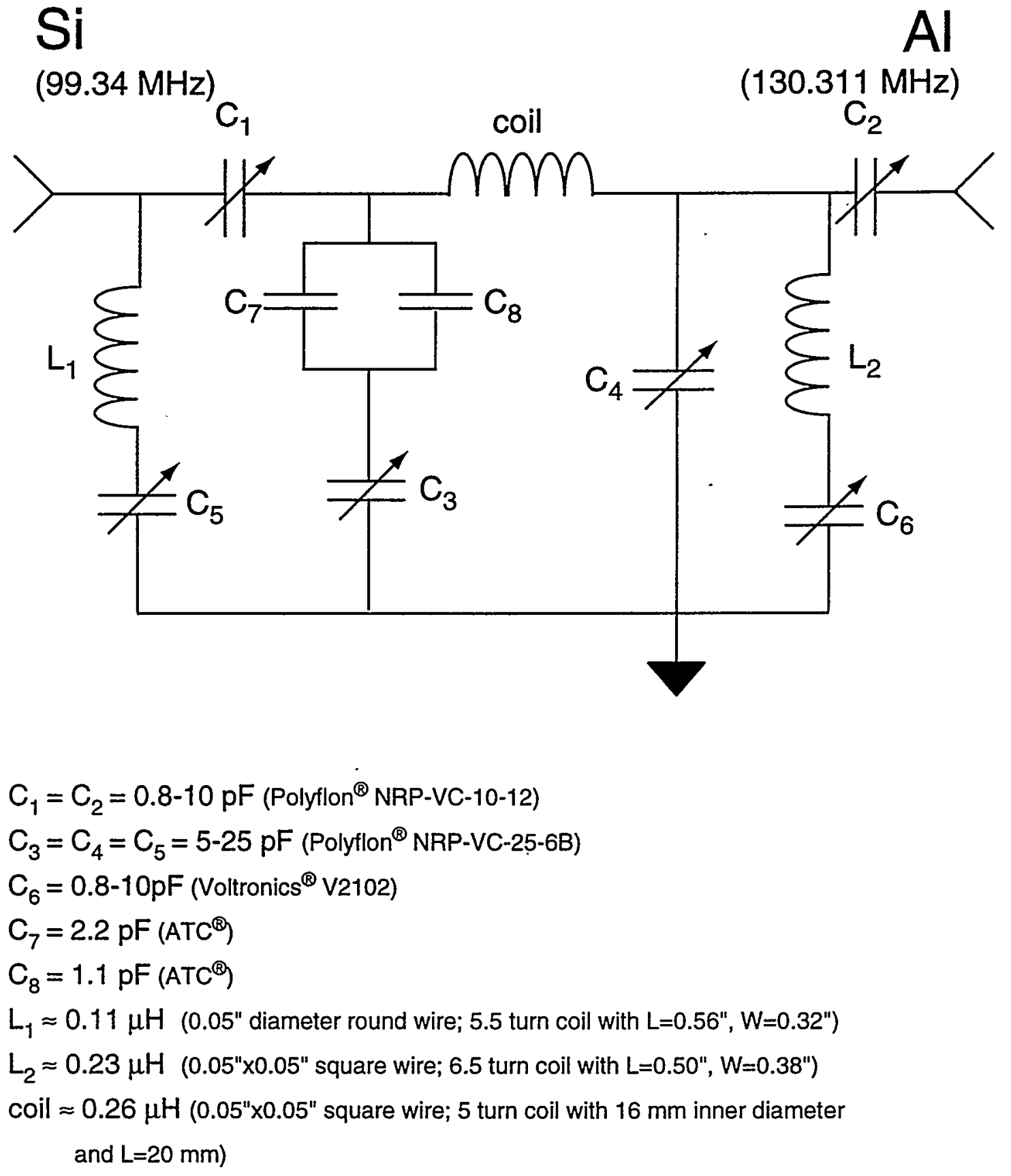

Figure 4.2 - Circuit diagram for the home-built double-resonance probe used in these experiments. The circuit is based on the "lumped-element" design of Doty et al..$^{91}$ except tunable traps are used on both channels. The impedances are estimated using the coil formula ${ }^{128.146}$ which neglects the effects of wire thickness and wire shape. At the power levels used for the CP experiments in this thesis, better than $40 \mathrm{~dB}$ isolation is observed on each channel. 
$\omega_{1 s^{\prime}} /(2 \pi) \approx 500 \mathrm{~Hz}$, which agrees well with the simulations of Figure $3.9 \mathrm{~b}$. The crosspolarization sequence used in these experiments incorporates spin-temperature alternation. ${ }^{47}$ For the contact-time dependence study, cross-polarization signal intensities were measured for contact times $\left(\tau_{\mathrm{CP}}\right)$ between $0.5 \mathrm{~ms}$ and $500 \mathrm{~ms}$. For these measurements, 128 scans of 1024 data points were summed with a recycle delay of $5 \mathrm{~s}$. Sixteen dummy scans preceded the collection of data. The silicon rotating-frame relaxation times $\left(T_{1 \rho}\right)$ were measured using the same parameters as for the $C P$ timedependence studies but adding a spin-lock period after the CP contact time (Figure 4.3). On-resonance measurements were made for each site in both the CP dynamics and silicon $T_{1 \rho}$ experiments to eliminate offset effects that are expected to occur at such low rf-power levels.

\subsection{Hartmann-Hahn Matching for Quadrupolar Nuclei}

Cross polarization is a process by which magnetization is transferred from one spin species to another. In solids, this typically occurs through the dipolar Hamiltonian although in some cases it can also occur through scalar J couplings. ${ }^{147}$ The basic types of cross polarization that have been developed so far are based on either adiabatic demagnetization or spin locking; ${ }^{7}$ only the second case will be discussed in this thesis.

To perform Hartmann-Hahn ${ }^{111}$ cross polarization, each spin species is subjected to rf irradiation in the form of a spin-lock field, and the amplitudes of these spin-lock fields are adjusted to permit energy-conserving magnetization transfer between the two types of spins. Mathematically, this can be described as follows.

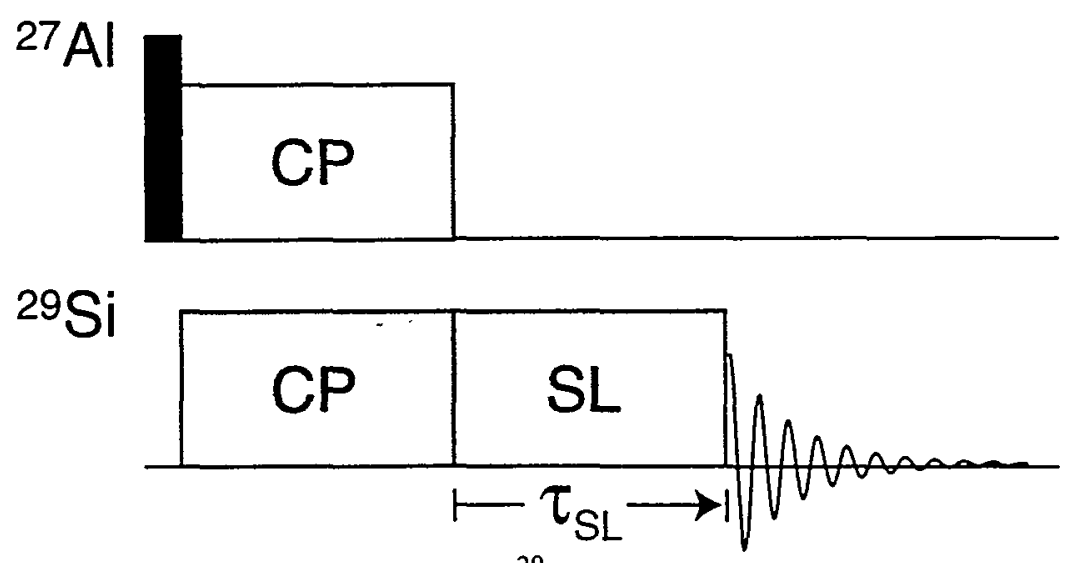

Figure 4.3 - Pulse sequence for measuring ${ }^{29} \mathrm{Si} \mathrm{T}_{1 \rho}$ values as described in the text. 
The Hamiltonian for cross polarization from many spins of type $S$ to a single spin of type $I$ is given (in the doubly rotating frame) by ${ }^{148,149}$

$$
\begin{aligned}
& \mathscr{H}=\sum_{\mathrm{k}} \hbar \Delta \omega_{\mathrm{S}_{\mathrm{k}}} \mathrm{S}_{\mathrm{kz}}+\hbar \omega_{1 \mathrm{~s}} \sum_{\mathrm{k}} \mathrm{S}_{\mathrm{kx}}+\hbar \Delta \omega_{\mathrm{I}} \mathrm{I}_{\mathrm{z}}+\hbar \omega_{1 \mathrm{I}} \mathrm{I}_{\mathrm{x}}+ \\
& +\hbar \sum_{\mathrm{k}} \mathrm{b}_{\mathrm{eff}, \mathrm{S}_{\mathrm{k}} \mathrm{I}}\left(2 \mathrm{~S}_{\mathrm{kz}} \mathrm{I}_{\mathrm{z}}\right) \\
& +\hbar \sum_{j>k} b_{e f f,} S_{j} S_{k}\left[2 S_{j z} S_{k z}-\frac{1}{2}\left(S_{j+} S_{k-}+S_{j-} S_{k+}\right)\right]
\end{aligned}
$$

where the effective dipolar coupling constants have the form (see Equations (1.68) and (1.71))

$$
b_{\text {eff, } s_{j} S_{k}}=-\left\{\left(\frac{\mu_{0}}{4 \pi}\right) \frac{\hbar \gamma_{S_{j}} \gamma_{S_{k}}}{r_{S_{j} S_{k}}^{3}}\right\} \frac{2}{\sqrt{6}} R_{2,0}^{D} .
$$

For the static case, $R_{2,0}^{D}$ will simply be proportional to the second-rank Legendre polynomial of the cosine of the angle between the dipolar vector and the $\mathrm{B}_{0}$ field while in the MAS case, it will contain terms that oscillate with $\omega_{\mathrm{r}}$ and $2 \omega_{\mathrm{r}}$ (see Equation (1.109))

$$
\begin{aligned}
b_{e f f, S_{j} S_{k}}^{\text {MAS }}=-\left\{\left(\frac{\mu_{0}}{4 \pi}\right)^{\hbar \gamma_{S_{j}} \gamma_{S_{k}}}\right. & \left.r_{S_{j} S_{k}}^{3}\right\}[ \\
& +\frac{1}{\sqrt{6}} D_{0,-2}^{(2)}\left(\alpha^{D}, \beta^{D}, \gamma^{D}\right) e^{i 2 \omega_{r} t} \\
& -\frac{1}{\sqrt{3}} D_{0,1}^{(2)}\left(\alpha^{D}, \beta^{D}, \gamma^{D}, \gamma^{D}\right) e^{-i \omega_{r} t} \\
& \left.+\frac{1}{\sqrt{6}} D_{0,2}^{(2)}\left(\alpha^{D}, \beta^{D}, \gamma^{D}\right) e^{-i 2 \omega_{r} t}\right] .
\end{aligned}
$$

Note that the choice of labels "I" and "S" in Equation (4.1) differs from the conventional usage but is consistent with the notation used in Chapter 3 .

For the case of on-resonance irradiation on both channels where $\left|\omega_{1 S}\right|$ "| $\left|b_{\text {eff, } s_{j} s_{k} \mid},\right| b_{\text {eff, } s_{k}} \mid$ and $\left|\omega_{11}\right| »\left|b_{\text {eff, } s_{k} I}\right|$, it is convenient to used a "tilted" interaction frame which corresponds a rotation of $\pi / 2$ about $\left(\sum_{\mathrm{k}} \mathrm{S}_{\mathrm{ky}}+\mathrm{I}_{\mathrm{y}}\right)$ (see Section $1.1 .2)^{148}$ 


$$
\begin{aligned}
\mathscr{H}^{\text {tilt }}= & \hbar \omega_{1 S} \sum_{k} S_{k z}+\hbar \omega_{1 I} I_{z}+\sum_{k} b_{e f f, S_{k} I} 2 S_{k x} I_{x} \\
& -\frac{1}{2} \hbar \sum_{j>k} b_{e f f, S_{j} S_{k}}\left[2 S_{j z} S_{k z}-\frac{1}{2}\left(S_{j+} S_{k-}+S_{j-} S_{k+}\right)\right] \\
& +\frac{3}{2} \hbar \sum_{j>k} b_{\text {eff, } S_{j} S_{k}}\left[\frac{1}{2}\left(S_{j+} S_{k+}+S_{j-} S_{k-}\right)\right]
\end{aligned}
$$

The last term in Equation (4.4) is non-secular and can usually be ignored. In this tilted frame, the direction of the spin lock on nucleus of a given type provides the axis of quantization for that nucleus.

For the moment, let us assume that all homonuclear dipolar couplings between the $\mathrm{S}$-spins can be ignored. (Their effects will be reintroduced below.) What we would like to see is how polarization can be transferred between the $S$ and I spins. Clearly, the third term on the right-hand side of Equation (4.4) is what couples the two species. Using Equations (1.52) and (1.53), it can be rewritten as

$$
\sum_{k} b_{e f f, S_{k} I} 2 S_{k x} I_{x}=\sum_{k} b_{e f f, S_{k} I}\left\{\frac{1}{2}\left(S_{k+} I+S_{k-} I_{+}\right)+\frac{1}{2}\left(S_{k+} I_{+}+S_{k-} I_{-}\right)\right\}
$$

Equation (4.5) contains two terms that can potentially be used for polarization transfer between the $S$ and I spin systems: (1) a zero-quantum or "flip-flop" term $\left(S_{k+} I_{-}+S_{k-} I_{+}\right)$ in which one spin flips down while the other flips up and (2) a double-quantum or "flopflop" term $\left(\mathrm{S}_{\mathrm{k}+} \mathrm{I}_{+}+\mathrm{S}_{\mathrm{k}-} \mathrm{I}_{-}\right)$in which either both spins flip up or both spins flip down.

If a quantum of S-spin magnetization equaled a quantum of I-spin magnetization, the "flip-flop" term would provide an energy-conserving mechanism for polarization transfer. Although the gyromagnetic ratios of different types of nuclei are not equal, it is possible to create a situation in which the quanta are the same by a judicious choice of rffield strengths. According to Hartmann and Hahn, ${ }^{11}$ transfer of polarization between two different types of nuclei in a static sample can occur if the rf-amplitudes are matched according to the condition

$$
\sqrt{I(I+1)-m_{I}\left(m_{I}-1\right)} \cdot\left|\omega_{1 I}\right|=\sqrt{S(S+1)-m_{S}\left(m_{S}-1\right)} \cdot\left|\omega_{1 S}\right|
$$


where $\omega_{1 \mathrm{I}}=-\gamma_{\mathrm{I}} \mathrm{B}_{1 \mathrm{I}}, \omega_{1 \mathrm{~S}}=-\gamma_{S} \mathrm{~B}_{1 \mathrm{~S}}$, and the transitions being matched are between quantum levels $\mathrm{m} \leftrightarrow(\mathrm{m}-1)$ for each nucleus. For two spin-1/2 nuclei, Equation (4.6) reduces to

$$
\left|\omega_{1 I}\right|=\left|\omega_{1 S}\right|
$$

while when polarization is transferred between a spin- $1 / 2$ nucleus (I) and the central transition of a half-integer quadrupolar nucleus (S), Equation (4.6) simplifies to

$$
\left|\omega_{11}\right|=\left(S+\frac{1}{2}\right) \cdot\left|\omega_{1 S}\right|
$$

Typically, the double-quantum term in Equation (4.5) -is ignored since it is nonsecular although in the case of small if fields and high spinning speeds, energy-conserving double-quantum cross polarization can occur between two spin-1/2 nuclei. ${ }^{150}$ However, it is interesting to note that because ${ }^{29} \mathrm{Si}$ has a negative gyromagnetic ratio while ${ }^{27} \mathrm{Al}$ has a positive one, the relevant term in the dipolar Hamiltonian for ${ }^{27} \mathrm{Al}-{ }_{\text {to- }}{ }^{29} \mathrm{Si}$ cross polarization is actually the "flop-flop" term. That is, the energy-conserving transition corresponds to one in which both spins flip the same way simultaneously (see Figure 4.4).

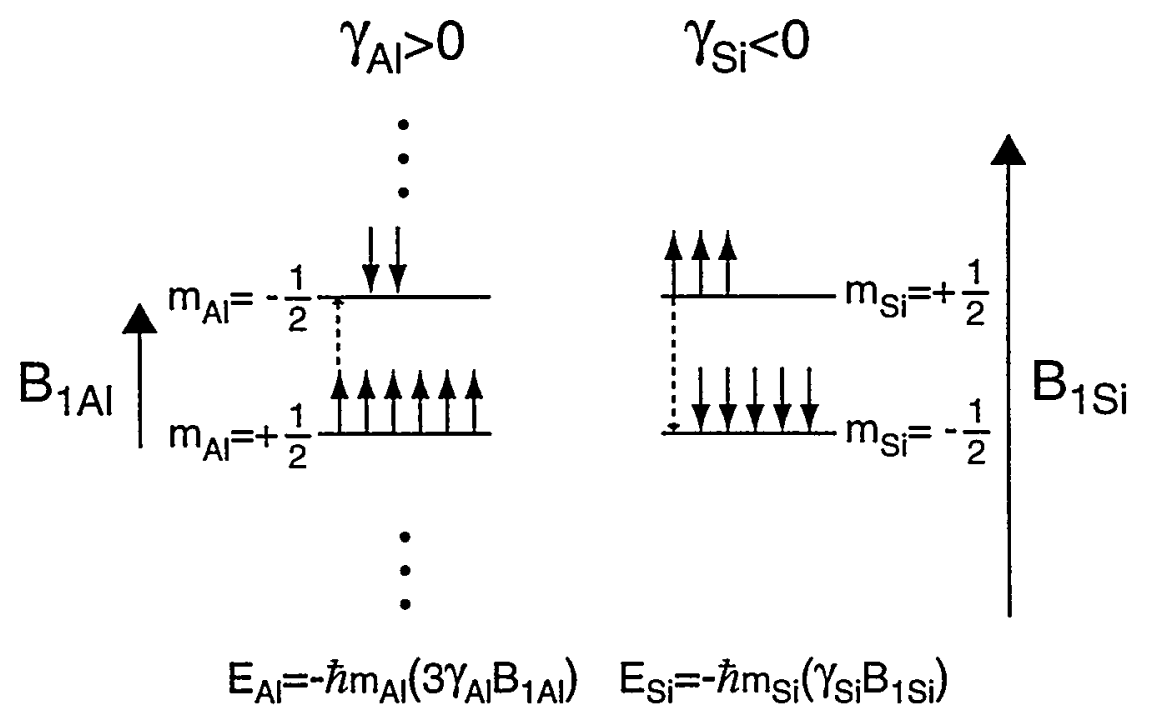

Figure 4.4 - Schematic of cross polarization from the central transition of ${ }^{27} \mathrm{Al}$ to ${ }^{29} \mathrm{Si}$ at the Hartmann-Hahn match. The opposite signs of the gyromagnetic ratios of the two spins causes the energy-conserving transition to be the "flop-flop" transition (e.g. - both spins change from spin up to spin down). 
This is merely a technicality, however, and the general concepts that have been developed for cross polarization between spins with positive gyromagnetic ratios will be applicable here.

When more than one nucleus of a given type is present in a sample, homonuclear dipolar couplings (the fourth term on the right-hand side of Equation (4.4)) will modify the effective spin-lock field felt by a given $S$ spin, and, therefore, a slightly different spinlock field strength $\left(\omega_{11}\right)$ will be required to match it. This leads to a broadening of the match condition of Equation (4.6) as depicted schematically in Figure 4.5a. Here, the number of crystallites fulfilling a given match condition is plotted as a function of the
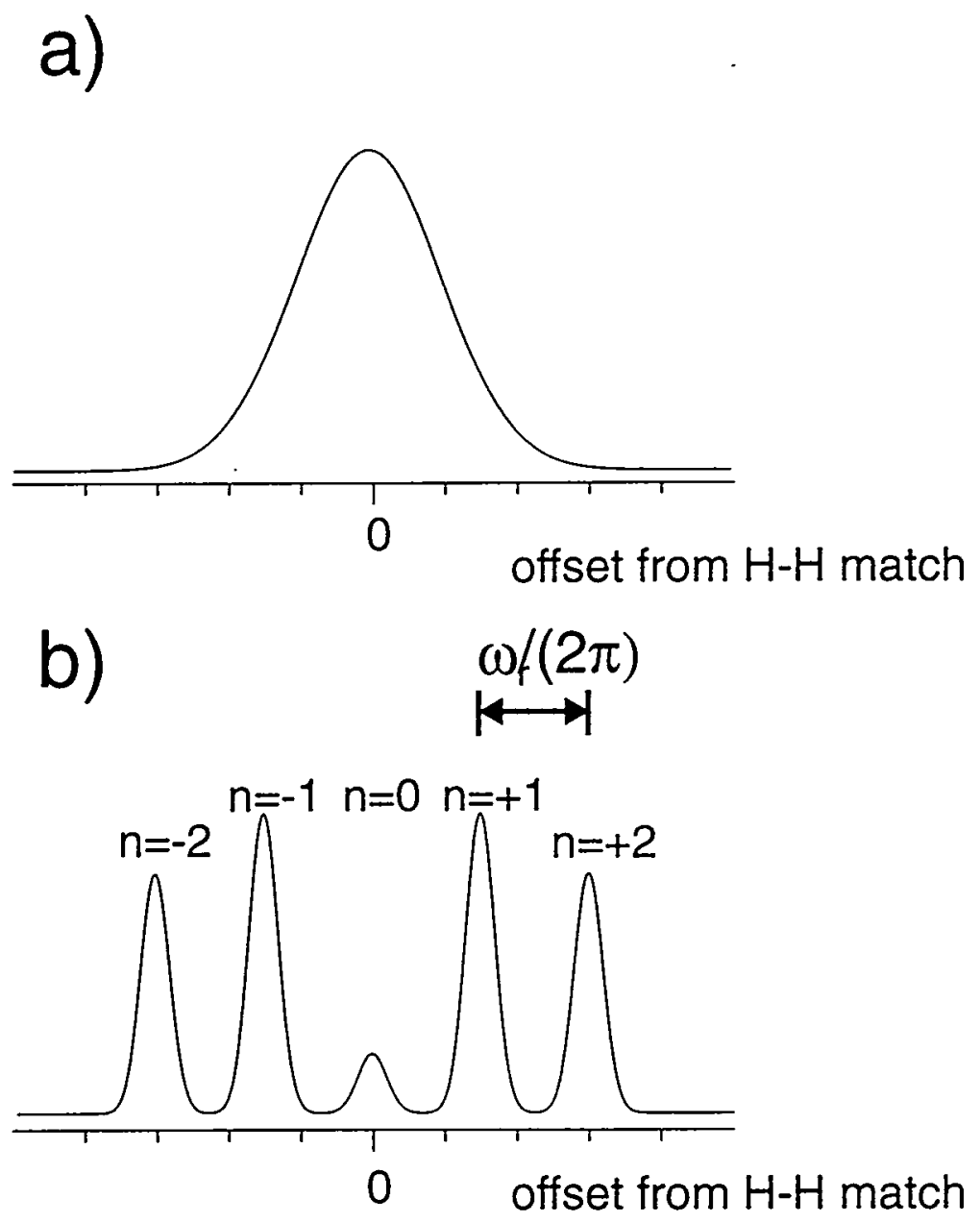

Figure 4.5 - (a) Schematic of the match condition for the static and "slow" spinning cases $\left(\omega_{\mathrm{r}} \ll \omega_{\mathrm{d}}\right)$. Intensities are plotted as a function of Hartmann-Hahn mismatch. Homonuclear dipolar couplings lead to a distribution of match conditions for different crystallites, centered about the Hartmann-Hahn match condition. (b) Schematic of the Hartmann-Hahn match condition for the "fast" spinning case $\left(\omega_{r}\right.$ " $\left.\omega_{d}\right)$. The match condition is split into sidebands spaced by $\omega_{r}$ 
deviation from the exact Hartmann-Hahn match: $\sqrt{I(I+1)-m_{I}\left(m_{I}-1\right)} \cdot\left|\omega_{11}\right|-\sqrt{S(S+1)-m_{S}\left(m_{S}-1\right)} \cdot\left|\omega_{1 S}\right| \cdot \quad$ Typical distributions for a static powder sample are symmetric and approximately Gaussian.

Magic-angle spinning further modifies the Hartmann-Hahn match condition. For the case of transfer between two spin-1/2 nuclei, it has been shown ${ }^{92,150}$ that when the rate of rotation, $\omega_{\mathrm{r}}$, equals or exceeds the strength of the homonuclear dipolar couplings, $\omega_{\mathrm{d}}$, the match condition of Equation (4.7) splits into distinct sidebands

$$
\left|\omega_{1 \mathrm{II}}\right|=\left|\omega_{1 \mathrm{~S}}\right|+n \omega_{\mathrm{r}}
$$

where $\mathrm{n}$ is an integer (see Figure 4.5b). These sidebands are a consequence of the fact that magic-angle spinning (see Equation (4.3)) makes the heteronuclear dipolar Hamiltonian time-dependent. Transforming Equation (4.4) to an interaction representation using

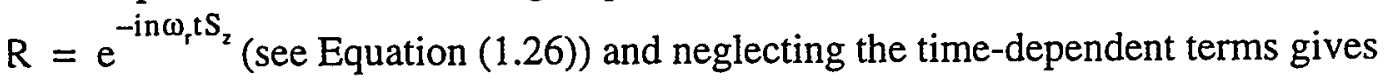

$$
\begin{aligned}
\left(\mathcal{H}^{\text {tilt }}\right)^{\prime}= & \hbar\left(\omega_{1 S}-n \omega_{r}\right) \sum_{k} S_{k z}+\hbar \omega_{1 I} I_{z} \\
& -\hbar d_{I S} \sum_{k} d_{-n, 0}^{(2)}(\theta) D_{0,-n}^{(1)}(\alpha, \beta, \gamma)\left[\frac{1}{2}\left(S_{k+} I_{-}+S_{k-} I_{+}\right)\right]
\end{aligned}
$$

where the homonuclear couplings have been ignored. For positive gyromagnetic ratios, it is clear that the match condition of Equation (4.9) permits energy-conserving magnetization transfer to occur via the Hamiltonian of Equation (4.10). To first order, Equation (4.10) is only valid for $\mathrm{n}= \pm 1, \pm 2$; thus, at short contact times the most efficient polarization transfer will occur for these. At longer contact times, however, equally efficient transfer occurs for the centerband due to higher-order processes, and less efficient transfer occurs for $|\mathrm{n}|>2 . .^{150,151} \quad \mathrm{~J}$ cross polarization can also potentially contribute to the centerband signal. ${ }^{147}$ As in the static case, the match conditions are broadened due to homonuclear couplings (see Figure 4.5) although the extent of broadening is reduced due to partial averaging by the sample spinning. ${ }^{92}$

An analogous modification of the static cross-polarization condition has been proposed for transfer between a spin-1/2 nucleus and the central transition of a halfinteger quadrupolar nucleus under MAS ${ }^{121}$ 


$$
\left|\omega_{11}\right|=(S+1 / 2)\left|\omega_{1 S}\right|+n \omega_{r}
$$

and has been observed experimentally. ${ }^{134}$ Compared to the aluminum homonuclear dipolar couplings in low albite $\omega_{\mathrm{d}} /(2 \pi) \approx 200 \mathrm{~Hz}$, a speed of $2400 \mathrm{~Hz}$ is in the "fast" spinning limit. Therefore, the match condition is expected to split into sidebands under our experimental conditions.

An additional complication occurs when $n \omega_{\mathrm{r}}$ is larger than the rf-field strength. This is depicted schematically in Figure 4.6 for the experimental conditions of Section 4.2. In these experiments the aluminum field strength was $\omega_{1 \mathrm{~s}} /(2 \pi) \approx 500 \mathrm{~Hz}$, and the spinning speed was $\omega_{\mathrm{r}} /(2 \pi)=2400 \mathrm{~Hz}$. From Equation (4.11), the centerband for cross polarization from ${ }^{27} \mathrm{Al}$ to ${ }^{29} \mathrm{Si}$ would be expected at a silicon field strength of $\omega_{1 \mathrm{I}} /(2 \pi)=1500 \mathrm{~Hz}$, the first positive $(\mathrm{n}=+1)$ sideband at $\omega_{1 \mathrm{I}} /(2 \pi)=3900 \mathrm{~Hz}$,

a)
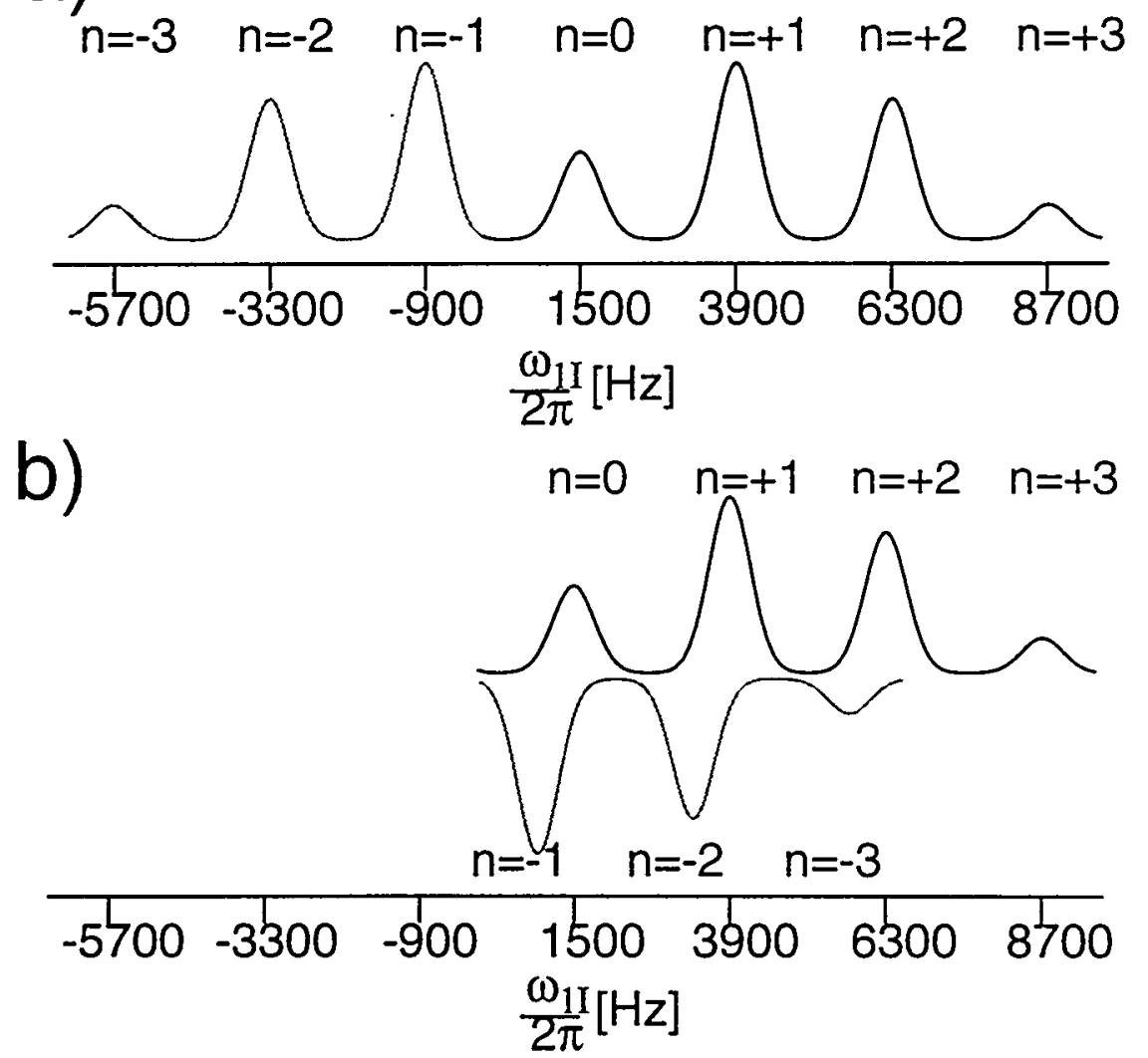

Figure 4.6 - Schematic of match conditions for our experiments as a function of the spinlock field strength on ${ }^{29} \mathrm{Si}$. (a) Hypothetical sideband positions based on Equation (4.11). (b) Folding-back of sidebands with negative intensity. 
the second positive $(n=+2)$ sideband at $\omega_{1 I} /(2 \pi)=6300 \mathrm{~Hz}$, etc. However, the $\mathrm{n}=-1$ and $\mathrm{n}=-2$ sidebands would be expected at negative field strengths (Figure 4.6a), which seems unphysical. Actually, a change in the sign of the field strength simply corresponds to a change in the direction of the quantization axis of one of the spins (i.e. - a shift from double-quantum to zero-quantum cross polarization). ${ }^{150}$ By using the Hamiltonian of Equation (4.10) and projecting the initial density matrix onto the Zeeman terms of this Hamiltonian, the final density matrix can be approximated. ${ }^{150}$ From this, one can determine that the cross-polarized signal in the vicinity of the Hartmann-Hahn match - condition will have the following dependence on the spin-lock amplitudes

$$
\left\langle I_{z}\right\rangle \propto \frac{N\left[(S+1 / 2)\left|\omega_{1 S}\right|+n \omega_{r}\right] \cdot\left(\left|\omega_{1 I}\right|\right)}{N\left[(S+1 / 2)\left|\omega_{1 S}\right|+n \omega_{r}\right]^{2}+\left(\left|\omega_{1 I}\right|\right)^{2}}
$$

The denominator in Equation (4.12) will always be positive, but the numerator will change sign depending on the relative magnitudes of $(S+1 / 2)\left|\omega_{1 S}\right|$ and $\omega_{r}$ This translates into a change in sign of the intensity of the cross-polarized signal. Thus, sidebands corresponding to negative values of $\omega_{11}$ will be folded back around the frequency $\omega_{1 \mathrm{I}} /(2 \pi)=0 \mathrm{~Hz}$ and will appear at $\left|\omega_{1 \mathrm{I}}\right|$ with negative intensity in the match condition spectrum (Figure 4.6b). ${ }^{150}$

Figure 4.7 shows the experimentally measured intensity of the signal crosspolarized from ${ }^{27} \mathrm{Al}$ to one of the ${ }^{29} \mathrm{Si}$ peaks in low albite as a function of the magnitude of the ${ }^{29} \mathrm{Si}$ rf field strength, $\omega_{1 \mathrm{II}} /(2 \pi)$, for cross-polarization contact times of $\tau_{\mathrm{CP}}=10,50$, and $750 \mathrm{~ms}$. The experiments agree reasonably well with the predicted match condition profile sketched in Figure 4.6b; however, the precise positions of the centerband and sidebands are difficult to determine due to both the partial overlap of positive and negative sidebands and the difficulty of measuring low field strengths accurately. All three peaks show similar behavior although the maxima are shifted since the offset is of the same order of magnitude as the ${ }^{27} \mathrm{Al}$ spin-lock field. The relative intensities of all sidebands remained the same (within experimental error) for contact times ranging between $10 \mathrm{~ms}$ and $750 \mathrm{~ms}$. 
The interference between different sideband matching conditions as seen in Figure 4.7 illustrates a second important consideration in setting up cross-polarization experiments involving half-integer quadrupolar nuclei at low $\mathrm{rf}$ powers. In addition to optimizing the quadrupolar spin-lock efficiency for a given spinning speed (as described in Chapter 3), one must make sure that the chosen spin-lock field strength and spinning speed create at least some sidebands of the match condition which do not interfere profoundly with other sidebands. Although the criteria for selecting efficient quadrupolar spin-lock field strengths eliminate some of the cases with the most destructive interference, there are other values of $\omega_{1 S}$ and $\omega_{\mathrm{r}}$ which give a good spin loc!. for the quadrupolar nucleus but for which the interference between the cross-polarization match sidebands would be severe.

Furthermore, $\omega_{\mathrm{r}}$ must be kept very stable since any change in the spinning speed will shift the positions of the folded-back sidebands and thereby alter the intensity of the cross-polarized signal (possibly even changing its sign). For our experimental parameters, slight changes in the spinning speed led to profound changes in the cross-polarized

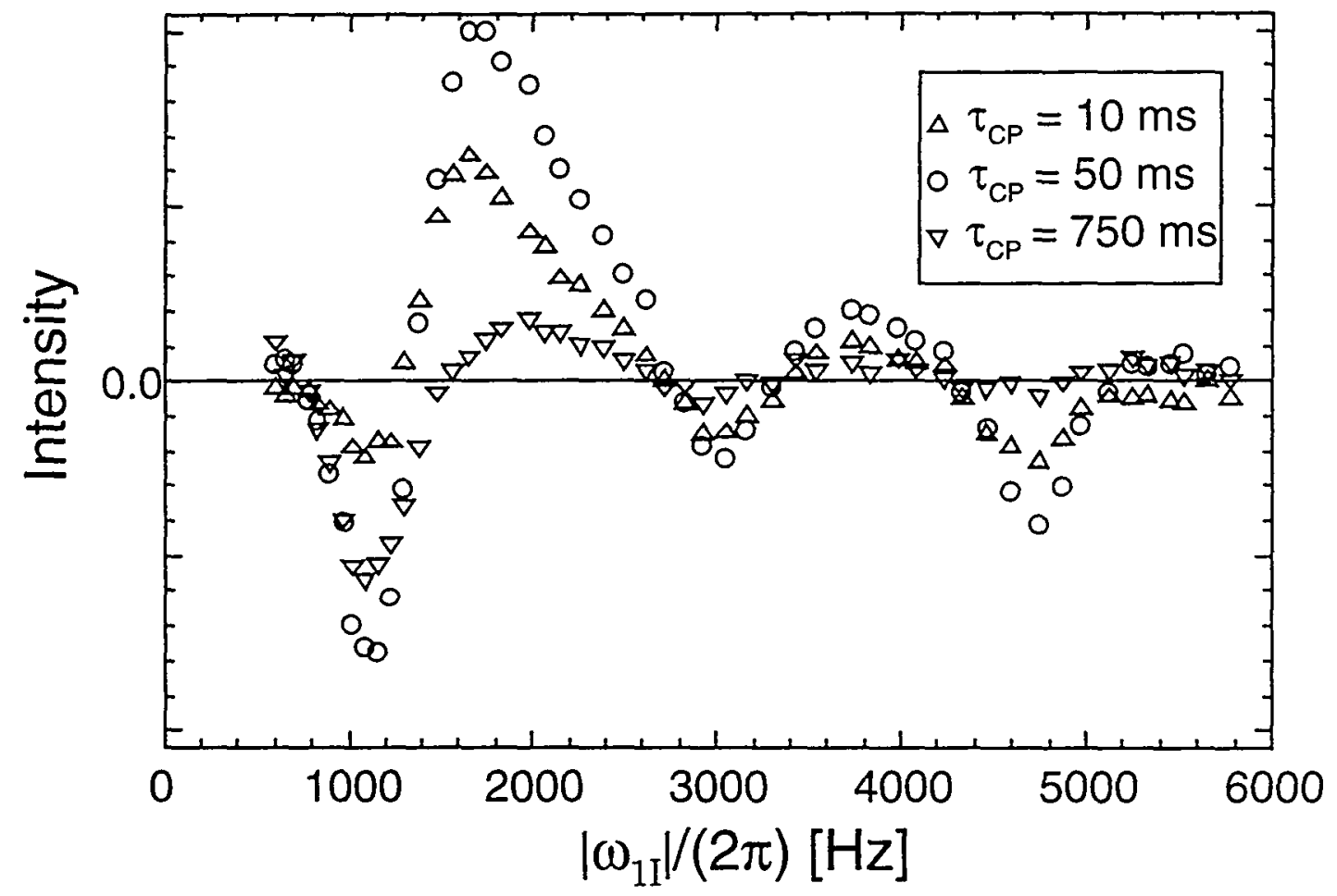

Figure 4.7 - Intensity of signal cross-polarized from ${ }^{27} \mathrm{Al}$ to the $\mathrm{Q}^{4}(2 \mathrm{Al}){ }^{29} \mathrm{Si}$ site in low albite. The pattern of matching condition sidebands agrees qualitatively with the expected pattern (see Figure 4.6b). 
intensities. The intensity of the peak at $-91.8 \mathrm{ppm}$ decreased by $34 \%$ when the spinning speed was changed from $2400 \mathrm{~Hz}$ to $2300 \mathrm{~Hz}$. Only a $2 \%$ decrease would be expected from varying the MAS rate alone (see Section 1.4.1); the rest must be a consequence of changes in the match condition. Even spinning-speed fluctuations of $10 \mathrm{~Hz}$ produced noticeable effects.

For both the CP contact-time dependence experiments and the silicon $T_{1 \rho}$ measurements, the centerband match condition was used. This match condition had relatively little overlap with the folded sidebands for the chosen values of $\omega_{1 S}$ and $\omega_{\mathrm{r}}$ as Figure 4.7 shows. Ncte that the centerband match signal may contain both high-order dipolar and J contributions; no attempt was made to distinguish between the two effects.

\subsection{Cross-Polarization Dynamics for Quadrupolar Nuclei}

In order to analyze the $\mathrm{CP}$ contact-time dependence of the ${ }^{29} \mathrm{Si}$ magnetization, the rotating-frame relaxation rate constants for all three silicon sites were measured onresonance at the if-field strength used for the cross-polarization experiments. Eight different time points from $\tau_{\mathrm{SL}}=500 \mathrm{~ms}$ to $\tau_{\mathrm{SL}}=6 \mathrm{~s}$ were measured four times each and fitted to a monoexponential decay as shown in Figure 4.8. The relaxation times that were obtained are: $\mathrm{T}_{1 \rho}(\delta=-91.8 \mathrm{ppm})=5.8 \pm 0.2 \mathrm{~s}, \mathrm{~T}_{1 \rho}(\delta=-96.1 \mathrm{ppm})=12.8 \pm 1.1 \mathrm{~s}$, and $T_{1 \rho}(\delta=-103.9 \mathrm{ppm})=11.5 \pm 0.7 \mathrm{~s}$. It is interesting to see that the silicon site with two aluminum nearest neighbors has a $T_{1 \rho}$ of only one-half the value of that for the silicon sites with only one aluminum nearest neighbor. This proportionality between the rate constant and the number of nearest neighbors suggests that the main relaxation pathway involves the aluminum atoms.

Figure 4.9 shows the cross-polarization intensity as a function of contact time for all three silicon sites. The measurements were done as three separate on-resonance measurements to avoid resonance-offset effects due to the low spin-lock field strengths. The most remarkable feature of these measurements is that the maximum polarization reached for the site with two aluminum nearest neighbors is lower than the maximum intensities of the two silicon sites with one aluminum nearest neighbor. The contact time dependences were fit to a simple model based on the so-called "thermodynamic" description of cross polarization described by Mehring. ${ }^{7}$ In this model (depicted 
schematically in Figure 4.10), the magnetization of a given spin species is parameterized in terms of a spin temperature. These species then interact with each other and with a "lattice" of other degrees of freedom according to first-order kinetic equations, subject to

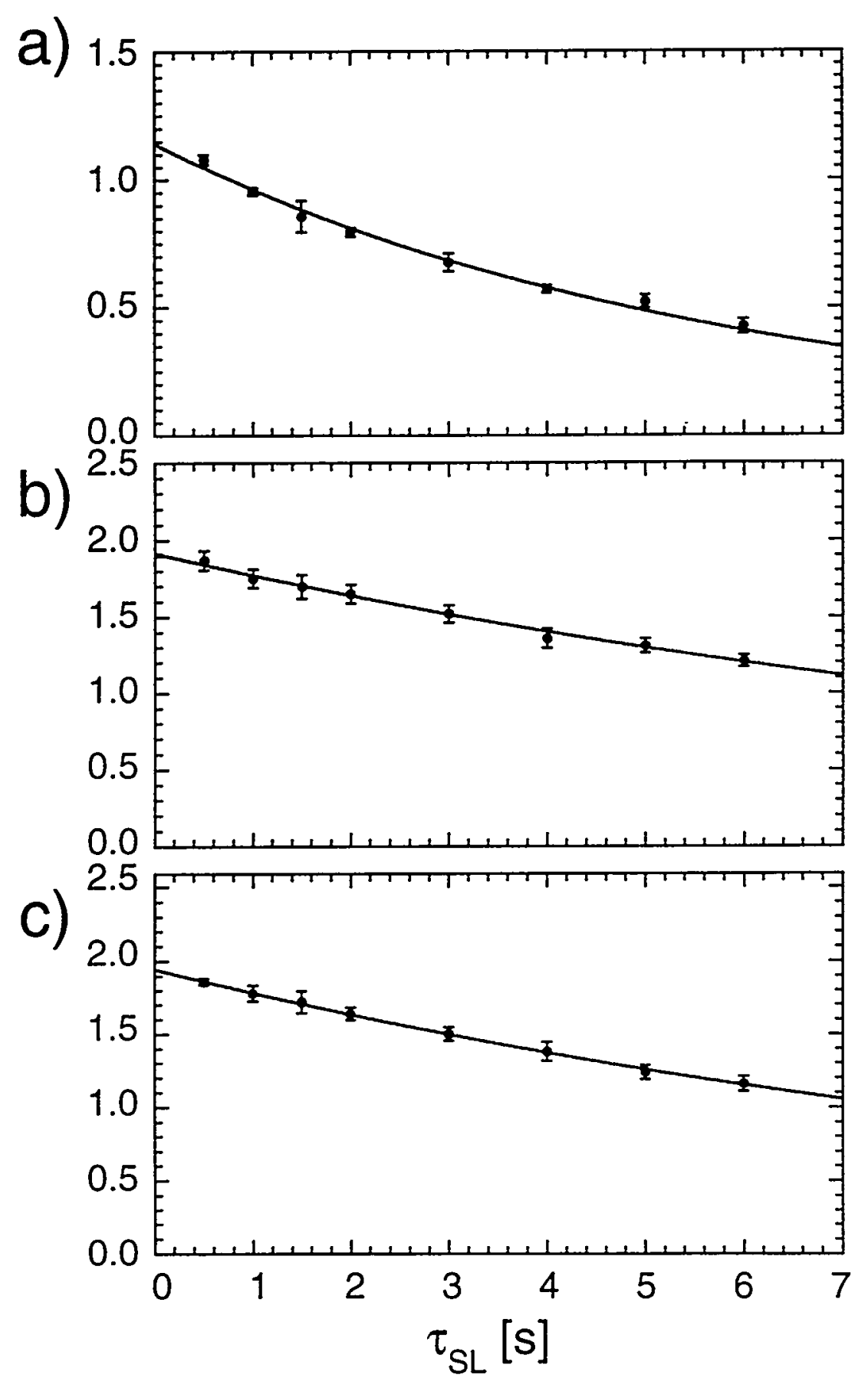

Figure $4.8-{ }^{29} \mathrm{Si} \mathrm{T}_{1 \mathrm{p}}$ measurements for the ${ }^{29} \mathrm{Si}$ sites in low albite. The error bars represent the standard deviation of four independent measurements. The solid lines are the best fits to the data points corresponding to $T_{1 \rho}$ values of (a) $T_{1 \rho}(\delta=-91.8 \mathrm{ppm})=5.8 \pm$ $0.2 \mathrm{~s}$, (b) $\mathrm{T}_{1 \rho}(\delta=-96.1 \mathrm{ppm})=12.8 \pm 1.1 \mathrm{~s}$, and $(\mathrm{c}) \mathrm{T}_{1 \mathrm{p}}(\delta=-103.9 \mathrm{ppm})=11.5 \pm 0.7 \mathrm{~s}$. 


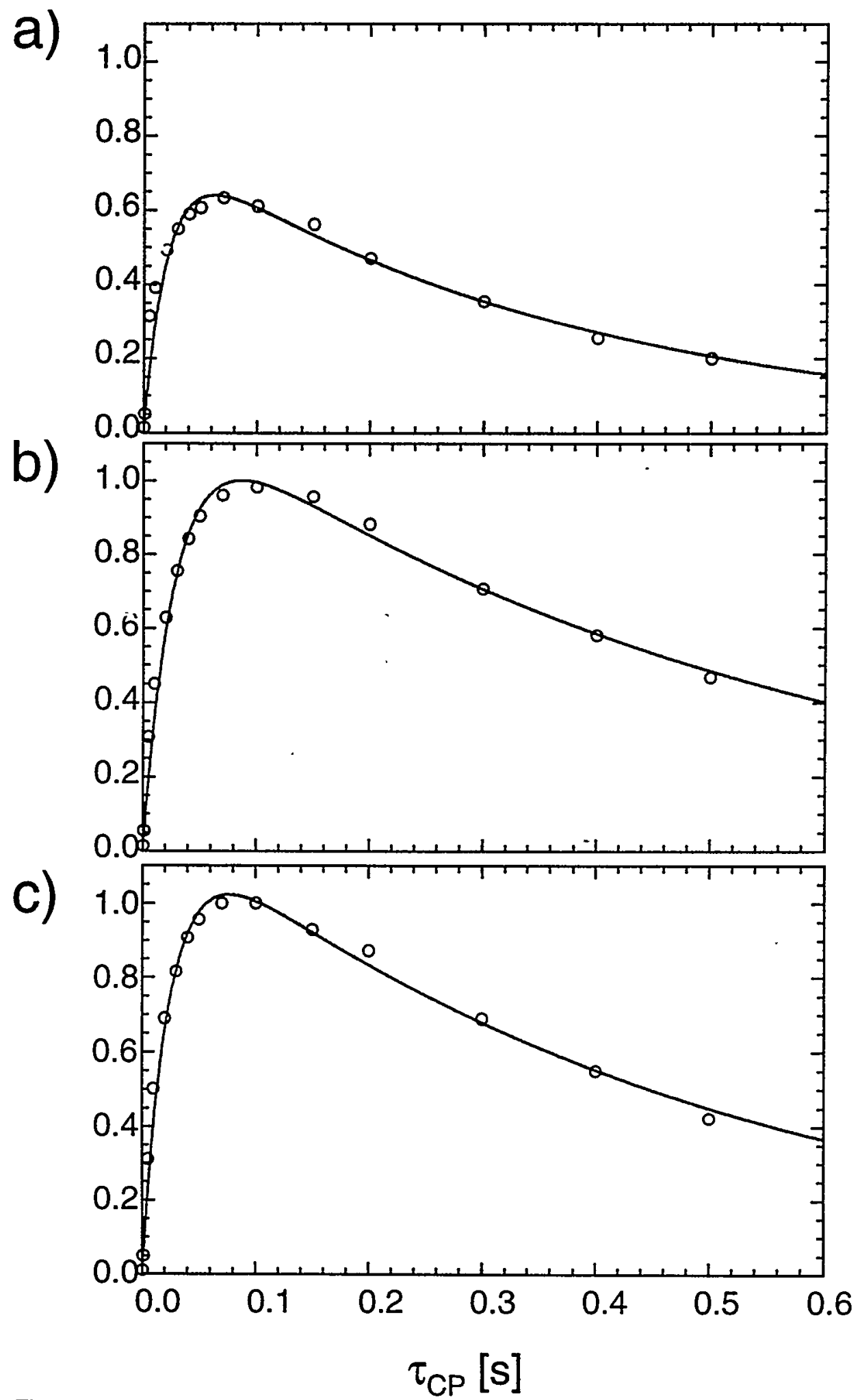

Figure 4.9 - Cross-polarization time dependences for the three different crystallographic silicon sites in low albite: (a) the $\mathrm{Q}^{4}(2 \mathrm{Al})$ site at $\delta=-91.8 \mathrm{ppm}$, (b) and (c) the $\mathrm{Q}^{4}(1 \mathrm{Al})$ sites at $\delta=-96.1 \mathrm{ppm}$ and $\delta=-103.9 \mathrm{ppm}$, respectively. The fits through the experimental points are based on Equation (4.34) and the parameters extracted from the fit are summarized in Table 4.1. 
the constraints imposed by the initial conditions and the law of conservation of energy. This simple model makes no assumptions about whether the mechanism for magnetization transfer is based on the dipolar-coupling interaction, the scalar-coupling interaction, or both. The derivation follows.

The quantum-mechanical definition of magnetization for $N_{I}$ spins of type $I$ is given by 5

$$
\left\langle\mathrm{M}_{\mathrm{z}}\right\rangle=\hbar \gamma_{\mathrm{I}} \operatorname{Tr}\left\{\rho \sum_{\mathrm{i}=1}^{\mathrm{N}_{1}} \mathrm{I}_{\mathrm{i}, \mathrm{z}}\right\}
$$

where the density matrix in the high-temperature approximation is given by Equation (1.16) and the Zeeman Hamiltonian is

$$
\mathcal{H}_{Z}=-\hbar \gamma_{\mathrm{I}} \mathrm{B}_{\mathrm{z}} \sum_{\mathrm{i}=1}^{\mathrm{N}_{\mathrm{l}}} \mathrm{I}_{\mathrm{i}, \mathrm{z}}
$$

Here we have used $B_{z}$ to indicate the magnetic field along which the spins are quantized. This may or may not be equal to the static field $\left(B_{0}\right)$ depending on the frame of reference. If the spins are assumed to be non-interacting (the validity of this approximation will be discussed below), Equation (4.13) can be written as $N_{I}$ multiplied by the magnetic moment of a single spin

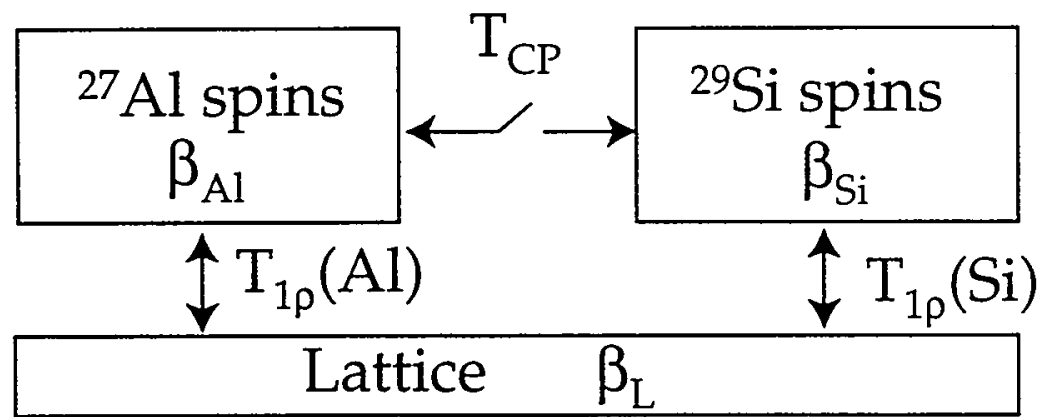

Figure 4.10 - Schematic of the phenomenological "thermodynamic" model of cross polarization which considers that each spin species can be described by a "spin temperature" and that both cross relaxation and relaxation to the "lattice" can be described by first-order kinetic equations. 


$$
\left\langle\mathrm{M}_{\mathrm{z}}\right\rangle=\mathrm{N}_{\mathrm{I}} \hbar \gamma_{\mathrm{I}} \operatorname{Tr}\left\{\rho \mathrm{I}_{\mathrm{z}}\right\}
$$

where the Zeeman Hamiltonian for a single spin (Equation (1.48)) is used in the density matrix. Substitution of Equations (1.16) and (1.48) into Equation (4.15) gives

$$
\left\langle M_{z}\right\rangle=N_{I} \hbar \gamma_{I} \cdot \frac{\left[\operatorname{Tr}\left\{I_{z}\right\}+\operatorname{Tr}\left\{\hbar \gamma_{I} B_{z} I_{z}^{2}\right\}\right]}{Z}
$$

where $\mathrm{Z}$ is the partition function (Equation (1.15)). The trace of the $I_{z}$ operator vanishes, and Equation (4.16) can therefore be written as

$$
\left\langle\mathrm{M}_{\mathrm{z}}\right\rangle=\beta_{\mathrm{I}} \mathrm{C}_{\mathrm{I}} \mathrm{B}_{\mathrm{z}}
$$

where the inverse spin temperature for the I spins is defined as

$$
\beta_{\mathrm{I}}=\frac{\hbar}{\mathrm{k}_{\mathrm{B}} \mathrm{T}_{\mathrm{I}}}
$$

The magnetization obeys the Curie law since it is inversely proportional to temperature, and $\mathrm{C}_{\mathrm{I}}$ is known as the Curie constant.

The Curie constant can be defined in terms of the following two functions

$$
F(I)=\frac{1}{Z}=\frac{1}{\operatorname{Tr}\{1\}}=\frac{1}{(2 I+1)}
$$

and

$$
G(I)=\operatorname{Tr}\left\{I_{z}^{2}\right\}=\sum_{m=-I}^{I} m^{2}=2 \sum_{m=1}^{I} m^{2}=\frac{I(I+1)(2 I+1)}{3} .
$$

By comparison of Equations (4.16) and (4.17), one can show that the Curie constant is given by

$$
C_{I}=N_{I} \hbar \gamma_{I}^{2} F(I) G(I)
$$


For spin-1/2 nuclei, $C_{I}$ is evaluated by direct substitution of $I=1 / 2$ into Equations (4.19) and (4.20). For cross polarization involving the central transition of a spin- $1 / 2$ nucleus, a modification must be made. ${ }^{135}$

To understand the nature of this modification, one must consider what effect selective excitation has on the density matrix. The equilibrium density operator for a spin$5 / 2$ nucleus in the lab frame is proportional to the $I_{z}$ operator and has the matrix form

$$
\rho_{\mathrm{eq}} \propto \frac{1}{2}\left[\begin{array}{cccccc}
5 & 0 & 0 & 0 & 0 & 0 \\
0 & 3 & 0 & 0 & 0 & 0 \\
0 & 0 & 1 & 0 & 0 & 0 \\
0 & 0 & 0 & -1 & 0 & 0 \\
0 & 0 & 0 & 0 & -3 & 0 \\
0 & 0 & 0 & 0 & 0 & -5
\end{array}\right]
$$

Application of a selective $90^{\circ}$ pulse along the $y$-axis will only affect the central transition. If the phase of this pulse and the receiver are alternated by $180^{\circ}$ between scans, ${ }^{47}$ the residual z-magnetization will be cancelled out and the density matrix during the spin lock can be approximated by a fictitious spin-1/2 operator on the central transition (see Section 1.2.5). ${ }^{120}$ Switching to the "doubly rotating tilted frame"152 in which the axis of quantization is along the effective field formed by the spin-lock field and the offset (see Figure 4.11) gives the matrix

$$
\rho\left(\tau_{S L}^{\text {alt, tilt }}\right) \propto \frac{1}{2}\left[\begin{array}{cccccc}
0 & 0 & 0 & 0 & 0 & 0 \\
0 & 0 & 0 & 0 & 0 & 0 \\
0 & 0 & 1 & 0 & 0 & 0 \\
0 & 0 & 0 & -1 & 0 & 0 \\
0 & 0 & 0 & 0 & 0 & 0 \\
0 & 0 & 0 & 0 & 0 & 0
\end{array}\right]
$$

Equation (4.23), not (4.22), is the form of the density matrix that must be substituted into Equation (4.15). It is effectively a spin- $1 / 2$ system which means that $I=1 / 2$ is that value that must be substituted into the equation for $G(\mathrm{I})$ (Equation (4.20)). However, the dimensionality of the matrix is still that of a spin- $5 / 2$ system so $I=5 / 2$ must be substituted into the equation for $\mathrm{F}(\mathrm{I})$ (Equation (4.19)). This gives a Curie constant of 


$$
C_{S}=N_{S} \hbar \gamma_{S}^{2} \cdot F(S=5 / 2) \cdot G(S=1 / 2)=\frac{N_{S} \hbar \gamma_{S}^{2}}{12}
$$

for the selectively excited aluminum atoms and a Curie constant of

$$
C_{I}=N_{I} \hbar \gamma_{I}^{2} \cdot F(I=1 / 2) \cdot G(I=1 / 2)=\frac{N_{I} \hbar \gamma_{I}^{2}}{4}
$$

for the silicon atoms during our cross-polarization experiments.

With this modification in mind, we can follow Mehring's treatment and address the dynamics of the cross-polarization process. The equilibrium aluminum magnetization on the central transition is given by

$$
\left\langle M_{S}\right\rangle_{\text {eq }}=\beta_{S}(t=\infty) C_{S} B_{0}
$$

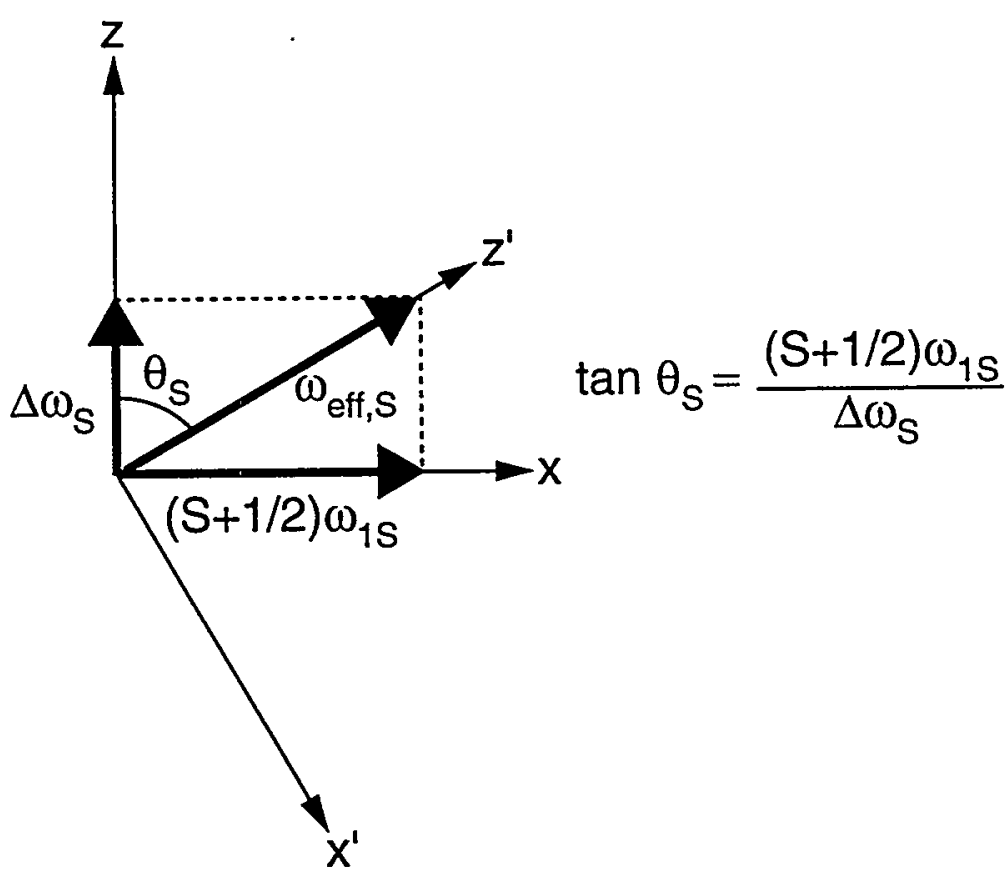

Figure 4.11 - Schematic of the doubly rotating tilted frame from the point of view of the $S$ spins. (The I spin case is analogous.) The $S$ spins see a coordinate system that rotates at the S-spin Larmor frequency while the I spins see a coordinate system that rotates at the Ispin Larmor frequency. Thus, only offset terms remain along the $z$ axis. The coordinate system is then "tilted" (by angles of $\theta_{S}$ and $\theta_{I}$ for the $S$ and I spins, respectively) so that the effective field in the rotating frame defines a new $z$ axis. For on-resonance irradiation, the tilting angle is $90^{\circ} .{ }^{152}$ 
where $C_{S}$ is given by Equation (4.24). This is the initial condition at the start of the experiment. After a selective pulse is applied, this magnetization is rotated by $90^{\circ}$ and spin-locked with a field of strength $B_{1 S}$. It is assumed that the pulse is short enough that no significant change in entropy will occur during the pulse. ${ }^{149}$ Note that since this pulse is selective, the effective field strength is actually $(\mathrm{S}+1 / 2) \mathrm{B}_{1 \mathrm{~S}} \cdot{ }^{27}$ Since the $\mathrm{B}_{1 \mathrm{~S}}$ field is much smaller than the $B_{0}$ field, a non-equilibrium situation exists. The origin of the time axis is chosen to be the point at which the spin-lock field is turned on. The inverse spin temperature at the start of the spin lock can be found by equating the magnetizations and solving for $\beta_{S}(t=0)$

$$
\begin{gathered}
\left\langle M_{S}(t=0)\right\rangle=\left\langle M_{S}\right\rangle_{\text {eq }} \\
\beta_{S}(t=0) C_{S}\left((S+1 / 2) B_{1 S}\right)=\beta_{S}(t=\infty) C_{S} B_{0} \\
\beta_{S}(t=0)=\frac{B_{0}}{(S+1 / 2) B_{1 S}} \cdot \beta_{S}(t=\infty) .
\end{gathered}
$$

From the form of Equation (4.27), one can see that the spins are cooled by the spinlocking process. They will, therefore, relax back to the lattice with a time constant $T_{1 \rho}^{(S)}$.

The Hartmann-Hahn match condition, however, has provided another relaxation pathway: the energy-conserving flip-flop transitions of Equation (4.5). In this phenomenological approach, it is assumed that the rate at which these transitions occur can be described by a cross relaxation rate constant, $T_{C P}$ The cross relaxation is constrained by the requirement of conservation of energy

$$
\frac{d\left(E_{S}\right)}{d t}+\frac{d\left(E_{I}\right)}{d t}=0
$$

Using the Curie law definition of magnetization (Equation (4.17)), Equation (4.28) becomes

$$
\frac{d\left(-\beta_{S} C_{S}\left((S+1 / 2) B_{1 S}\right)^{2}\right)}{d t}+\frac{d\left(-\beta_{I} C_{I}\left((I+1 / 2) B_{1 I}\right)^{2}\right)}{d t}=0
$$


where a generalized version of the Hartmann-Hahn condition has been used to describe the effective applied field strengths. Substitution of the Curie constants gives

$$
\frac{d \beta_{S}}{d t}+\lambda\left(\frac{\gamma_{I} B_{1 I}}{3 \gamma_{S} B_{1 S}}\right)^{2} \cdot \frac{d \beta_{I}}{d t}=0
$$

where

$$
\lambda=\frac{(\mathrm{S}+1 / 2) \mathrm{N}_{\mathrm{I}}}{(\mathrm{I}+1 / 2) \mathrm{N}_{\mathrm{S}}}
$$

When the Hartmann-Hahn match condition is met, the term in parentheses in Equation (4.30) equals one.

Using the constraint of Equation (4.30), one can write first-order rate equations that describe the cross polarization process

$$
\frac{\mathrm{d}}{\mathrm{dt}}\left[\begin{array}{c}
\beta_{S} \\
\beta_{\mathrm{I}}
\end{array}\right]=\left[\begin{array}{cc}
-\mathrm{k}_{\mathrm{S}}-\lambda \mathrm{k}_{\mathrm{SI}} & \lambda \mathrm{k}_{\mathrm{SI}} \\
\mathrm{k}_{\mathrm{SI}} & -\mathrm{k}_{\mathrm{I}}-\mathrm{k}_{\mathrm{SI}}
\end{array}\right]\left[\begin{array}{c}
\beta_{S} \\
\beta_{\mathrm{I}}
\end{array}\right]
$$

where $\mathrm{k}_{\mathrm{I}}$ and $\mathrm{k}_{\mathrm{S}}$ are the relaxation rate constants for the $\mathrm{I}$ and $\mathrm{S}$ spins respectively and $\mathrm{k}_{\mathrm{SI}}$ is the cross-polarization rate constant. Using the initial conditions of

$$
\begin{aligned}
& \beta_{\mathrm{I}}(0)=0 \\
& \beta_{S}(0)=S_{0}
\end{aligned}
$$

where $S_{0}$ is a constant representing the initial S-spin magnetization (see Equation (4.27)), one can solve Equation (4.32) to obtain the following equation for the intensity of the I spin as a function of contact time

$$
\begin{aligned}
& I\left(\tau_{C P}\right)=\frac{S_{0} \xi k_{S I}}{W} \cdot\left[e^{-\left(k_{s}+k_{1}+k_{S I}(1+\lambda)-W\right) \tau_{C P} / 2}\right. \\
& \left.-\mathrm{e}^{-\left(\mathrm{k}_{\mathrm{S}}+\mathrm{k}_{\mathrm{I}}+\mathrm{k}_{\mathrm{SI}}(1+\lambda)+\mathrm{W}\right) \tau_{\mathrm{CP}} / 2}\right]
\end{aligned}
$$

where 


$$
W=\sqrt{\left(k_{S}-k_{I}-k_{S I}(1-\lambda)^{2}\right)+4 k_{S I}^{2} \lambda} \text {. }
$$

Note that Equation (4.34) does not make any simplifying assumptions about the relative magnitudes of the different parameters. Although in our case $\mathrm{k}_{\mathrm{I}}$ is several orders of magnitude smaller than $\mathrm{k}_{\mathrm{S}}$ and $\mathrm{k}_{\mathrm{SI}}$, we chose not to make any approximations based on this fact since $\mathrm{k}_{\mathrm{I}}$ could be independently and directly measured. It is also important to realize that assumptions which may be valid for systems with an abundance of protons (such as the assumption that $\lambda$ is small) will often not be appropriate for systems that do not contain protons. Unwarranted simplifications have been made in the literature ${ }^{134}$ and may have led to incorrect conclusions.

This phenomenological theory of cross polarization has several limitations. It assumes that a common spin temperature is rapidly reached among spins of a given type even though this may not be true for a system of dilute spins with a small gyromagnetic ratio (where heteronuclear and homonuclear couplings are of the same magntitude). ${ }^{148}$ Thermodynamics also cannot be rigorously applied to isolated pairs of spins. Another problem with the phenomenological approach is that it incorrectly describes the behavior of the system when the spin-lock amplitudes are mismatched. ${ }^{149}$ A more rigorous treatment of cross polarization ${ }^{148,149}$ has shown that, due to quantum-mechanical constraints, a system will often not reach its true equilibrium state but rather a metastable quasi-equilibrium. The quasi-equilibrium approach provides a more accurate description of cross polarization under mismatched conditions and predicts a broadened match condition (see Figure 4.5a) although the predicted lineshape is Lorentzian rather than the Gaussian typically observed. The quasi-equilibrium approach does not address crosspolarization dynamics, however. Still another concern (particular to our situation) is that the fictitious spin-1/2 approximation may break down for quadrupolar nuclei.

Despite its deficiencies, the phenomenological model has been widely applied with some degree of success to studies of $\mathrm{CP}$ dynamics at the Hartmann-Hahn match which leads us to consider it here. An important caveat is that parameters extracted from this model should not be viewed as true thermodynamic variables but as dependent in some (unspecified) way on the experimental conditions. 
Equation (4.34) can now be compared with the experimentally measured curves for cross polarization dynamics from ${ }^{27} \mathrm{Al}$ to ${ }^{29} \mathrm{Si}$ in low albite. For the silicon sites with one aluminum nearest neighbor, $\lambda$ is equal to 3 (see Equation (4.31)) while for the silicon site with two aluminum nearest neighbors, $\lambda$ equals $3 / 2$. Although most of the intensity in the ${ }^{29} \mathrm{Si}$ MAS spectrum of low albite at $\omega_{\mathrm{r}} /(2 \pi)=2400 \mathrm{~Hz}$ is concentrated in the centerbands, a few low-intensity sidebands are present for each site. Thus, a small correction factor, $\xi$, is included for each site so that the centerband intensities can be directly compared. $\xi$ represents the fraction of the total intensity of a given site that is in the centerband. Because the principal values of the ${ }^{29} \mathrm{Si}$ chemical shielding tensors in low albite are known (see Chapter 5), ${ }^{153} \xi$ can be calculated. At $\omega_{\mathrm{r}^{\prime}} /(2 \pi)=2400 \mathrm{~Hz}, \xi$ is equal to $0.76,0.83$, and 0.81 for the $\delta=-91.8 \mathrm{ppm}, \delta=-96.1 \mathrm{ppm}$, and $\delta=-103.9 \mathrm{ppm}$ sites respectively. These values are quite similar to each other and show that for our case, this is indeed a small correction. However, for slowly-spun samples that contain sites with vastly different chemical-shielding anisotropies, the differences in $\xi$ would be significant.

To determine the cross-polarization time dependences, the measured values (see Figure 4.8) of the silicon rotating-frame relaxation times $\left(k_{I}=1 / T_{1 p}^{(1)}\right)$ were used, and three-parameter fits were performed to extract values for $\mathrm{S}_{0}, \mathrm{k}_{\mathrm{S}}$, and $\mathrm{k}_{\mathrm{SI}}$. The results of the best fits are summarized in Table 4.1. The rate constants for the cross-polarization process

\begin{tabular}{|c|c|c|c|c|}
\hline $\begin{array}{c}\text { Isotropic Shift } \\
{[\mathbf{p p m}]}\end{array}$ & $\begin{array}{c}\mathbf{k}_{\mathbf{I}}=\mathbf{1} / \mathbf{T}_{\mathbf{1}}{ }^{(\mathbf{I})} \\
{\left[\mathbf{s}^{-\mathbf{1}}\right]^{\mathbf{a}}}\end{array}$ & $\begin{array}{c}\mathbf{k}_{\mathbf{S}}=\mathbf{1} / \mathbf{T}_{\mathbf{1}}{ }^{(\mathbf{S})} \\
{\left[\mathbf{s}^{-\mathbf{1}}\right]}\end{array}$ & $\begin{array}{c}\mathbf{k}_{\mathbf{S I}}=\mathbf{1} / \mathbf{T}_{\mathbf{C P}} \\
{\left[\mathbf{s}^{-1}\right]}\end{array}$ & $\mathbf{S}_{\mathbf{0}}$ \\
\hline \hline-91.8 & 0.17 & 45 & 2.8 & 17 \\
\hline-96.1 & 0.08 & 29 & 2.2 & 22 \\
\hline-103.9 & 0.09 & 33 & 2.4 & 25 \\
\hline
\end{tabular}

Table 4.1 - Parameters from cross-polarization time-dependence fits

a. $T_{1 \rho}{ }^{(I)}$ 's for silicon were measured by independent experiments and used as fixed parameters.

are, as expected, all in the same range since the distances to the nearest-neighbor aluminum atoms are very similar (see Table 3.1). However, the initial S-spin 
magnetizations and the aluminum relaxation rate constants show a rather large and unexpected variation. Since there is only one aluminum site, one would expect to obtain similar values for these two parameters.

Although there is only one crystallographic aluminum site in low albite, it is conceivable that there could be different $T_{1 p}$ relaxation rates for aluminum atoms in different magnetic environments. Figure 4.12a shows a schematic view of the two possible environments, an $S_{2} I$ system (for $Q^{4}(2 \mathrm{Al})$ sites) and an SI system (for $\mathrm{Q}^{4}(1 \mathrm{Al})$ sites). To determine if the magnetic environments were a significant factor, aluminum $T_{1 \rho}$

a)
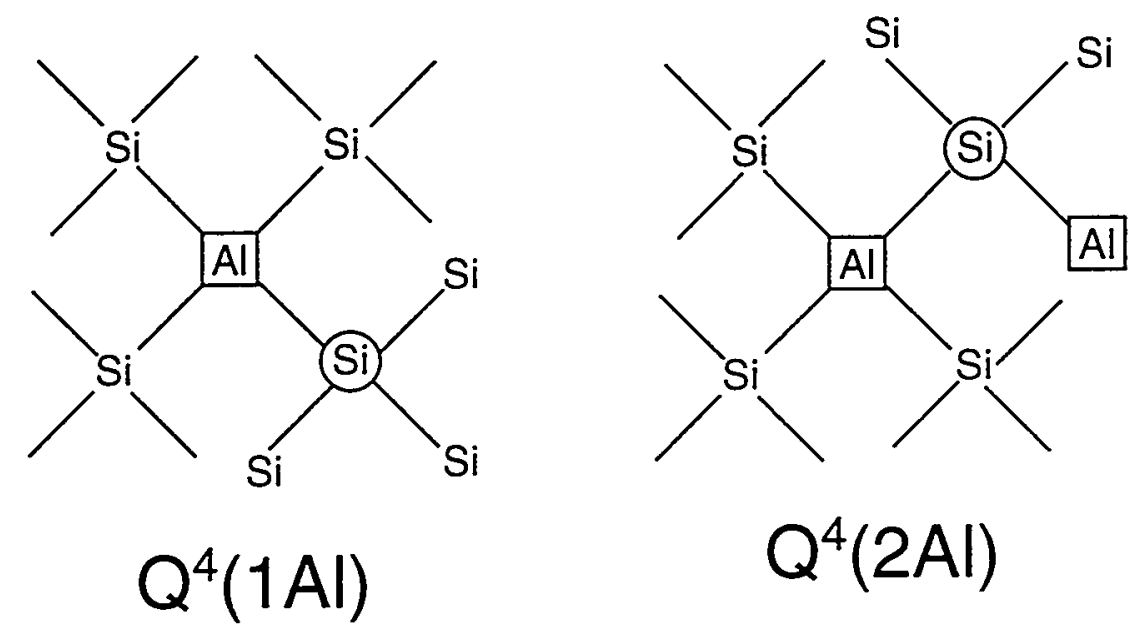

b)

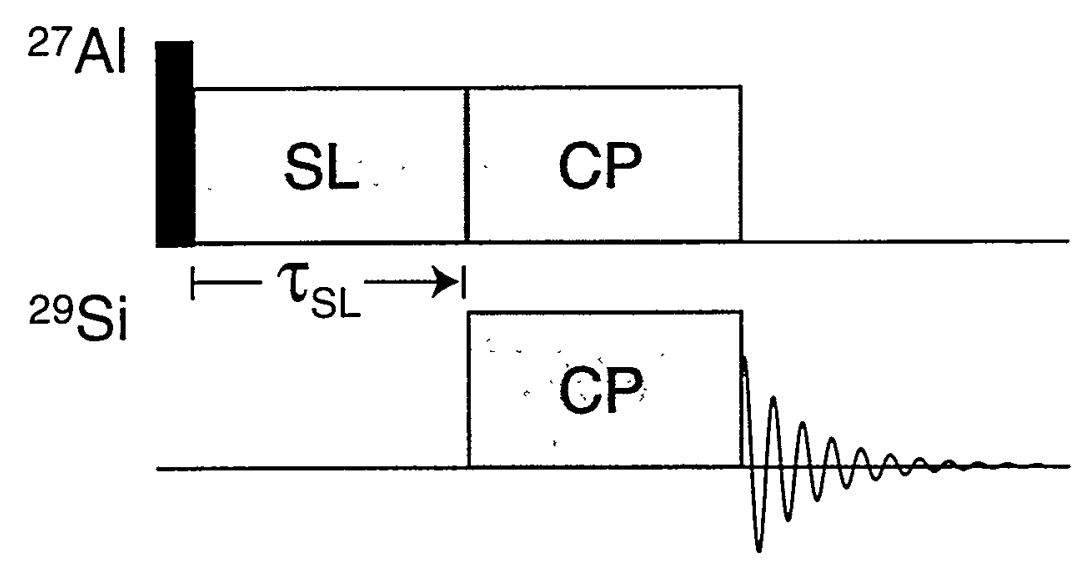

Figure 4.12 - (a) Possible magnetic environments in low albite. (b) Pulse sequence for indirect ${ }^{27} \mathrm{Al} \mathrm{T} \mathrm{T}_{10}$ measurement. A spin-lock on the ${ }^{27} \mathrm{Al}$ channel is followed by cross polarization to ${ }^{29} \mathrm{Si}$ to separately measure the $\mathrm{T}_{1 \rho}$ 's of aluminum atoms in different magnetic environments. 
values were measured indirectly using to the pulse sequence of Figure $4.12 \mathrm{~b}$. The results of such measurements are shown in Figure 4.13. The fast-decaying portion of the biexponential (see Section 3.4) could not be reliably measured due to the finite rise time of the pulse on the ${ }^{29} \mathrm{Si}$ channel, but the slow time constants are the same for all three sites to within experimental error. Variations in aluminum $T_{1 \rho}$ values are, therefore, not the reason for the low intensity of the $\mathrm{Q}^{4}(2 \mathrm{Al})$ site.

Another potential method for deconvolving the rate parameters would be to perform "drain" experiments as a function of contact time. ${ }^{143,154}$ Such experiments would consist of two parts: (a) Hartmann-Hahn cross polarization from ${ }^{29} \mathrm{Si}$ to ${ }^{27} \mathrm{Al}$ followed by detection of the ${ }^{29} \mathrm{Si} \mathrm{FID}$, and (b) a spin-lock only on the ${ }^{29} \mathrm{Si}$ spins followed by detection of the ${ }^{29} \mathrm{Si} \mathrm{FID}$. Phase modulation of the spin lock on the ${ }^{27} \mathrm{Al}$ spins during part (a) would prevent ${ }^{27} \mathrm{Al}-{ }_{\text {-to- }}{ }^{29} \mathrm{Si}$ polarization transfer and eliminate the effect of the ${ }^{27} \mathrm{Al} \mathrm{T} \mathrm{T}_{1 \rho}$ on the signal. Taking the difference between experiments (b) and (a) and normalizing to experiment (b) would give a signal with an intensity that depends only on $\lambda$ and $T_{C P} A$

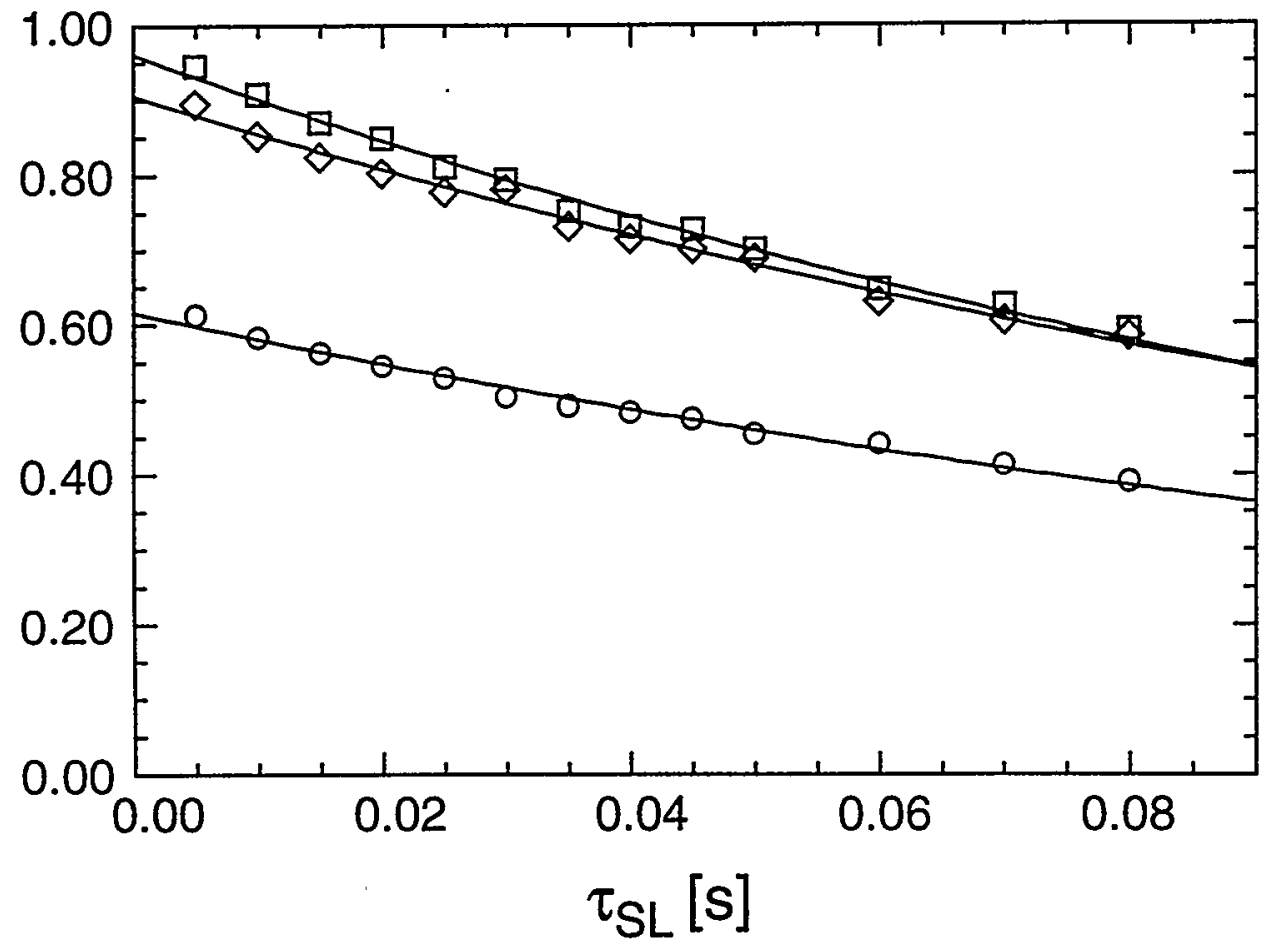

Figure 4.13 - Indirect $T_{1 \rho}$ measurements for the aluminum atoms near the three different silicon sites in low albite. The circles correspond to the $\delta=-91.8 \mathrm{ppm}$ site; the diamonds correspond to the $\delta=-96.1 \mathrm{ppm}$ site; and the squares correspond to the $\delta=-103.9 \mathrm{ppm}$ site. To within the accuracy of the measurement, the slow time constants are the same in all three cases. 
single-parameter fit could therefore be used, permitting a more reliable determination of $\mathrm{T}_{\mathrm{CP}}$ Unfortunately, the long $\mathrm{T}_{1}$ of the ${ }^{29} \mathrm{Si}$ spins makes ${ }^{29} \mathrm{Si}$-to- ${ }^{27} \mathrm{Al}$ cross polarization impractical.

To determine how sensitive the contact-time-dependence fits are to the parameters, Equation (4.34) was used to generate simulated curves for several sets of parameters. Specifically, pairs of curves with identical values of $S_{0}$ and $k_{S}$ (the mean values of those listed in Table 4.1) were generated for $\lambda=3 / 2$ (the $\mathrm{Q}^{4}(2 \mathrm{Al})$ site) and for $\lambda=3$ (the $\mathrm{Q}^{4}(1 \mathrm{Al}$ ) sites). The other parameters were varied but were constrained to have the same order of magnitude as the values in Table 4.1. A few of these curves are plotted in Figure 4.14a-d with the dotted lines corresponding to the $\mathrm{Q}^{4}(2 \mathrm{Al})$ site and the solid lines corresponding to one of the $\mathrm{Q}^{4}(1 \mathrm{Al})$ sites. In Figure $4.14 \mathrm{a}$, the $\mathrm{k}_{\mathrm{I}}$ and $\mathrm{k}_{\mathrm{SI}}$ values from Table 4.1 were used (along with the appropriate values of $\xi$ ), and the maximum $\mathrm{CP}$ intensity for the site with two aluminum nearest neighbors was found to be higher than that for the siie with one aluminum nearest neighbor. However, the maxima of these simulated curves appeared especially sensitive to the value of $k_{S I}$. With just slight variations in the value of $k_{S I}$ (Figure $4.14 \mathrm{~b}-\mathrm{d}$ ), the relative intensities of the $\mathrm{Q}^{4}(2 \mathrm{Al})$ and the $\mathrm{Q}^{4}(1 \mathrm{Al})$ sites changed considerably. In most cases, they were within a few percent of each other, but sometimes the $Q^{4}(2 \mathrm{Al})$ site was more intense than the $\mathrm{Q}^{4}(1 \mathrm{Al})$ site and other times the converse was true. Thus, there is no simple relationship between the maximum intensity of the crosspolarized signal and the number of nearest-neighbor aluminum atoms for the conditions of our experiments.

In view of these simulations, our experimental observation that the maximum intensity reached for the site with two aluminum nearest neighbors is lower than the maximum intensities of the two silicon sites with one aluminum nearest neighbor is not surprising and agrees qualitatively with the phenomenological theory. In fact, precise quantitative agreement between our data and Equation (4.34) is not expected. The spintemperature model we have used assumes an exponential decay of the spin-locked magnetization, but as pointed out in Chapter 3 , the relaxation of ${ }^{27} \mathrm{Al}$ in low albite is at least biexponential. The fact that there is no simple way to incorporate biexponential relaxation processes is one of the limitations of the phenomenological model. However, Equation (4.34) is still a useful approximation. The values of $T_{1 \rho}^{(S)}$ extracted from the fits 
(see Table 4.1) all fall into the physically reasonable range of being larger than the fastdecaying component and smaller than the slowly-decaying component of the measured rotating-frame relaxation of ${ }^{27} \mathrm{Al}$ in low albite (see Figure 3.15 ).
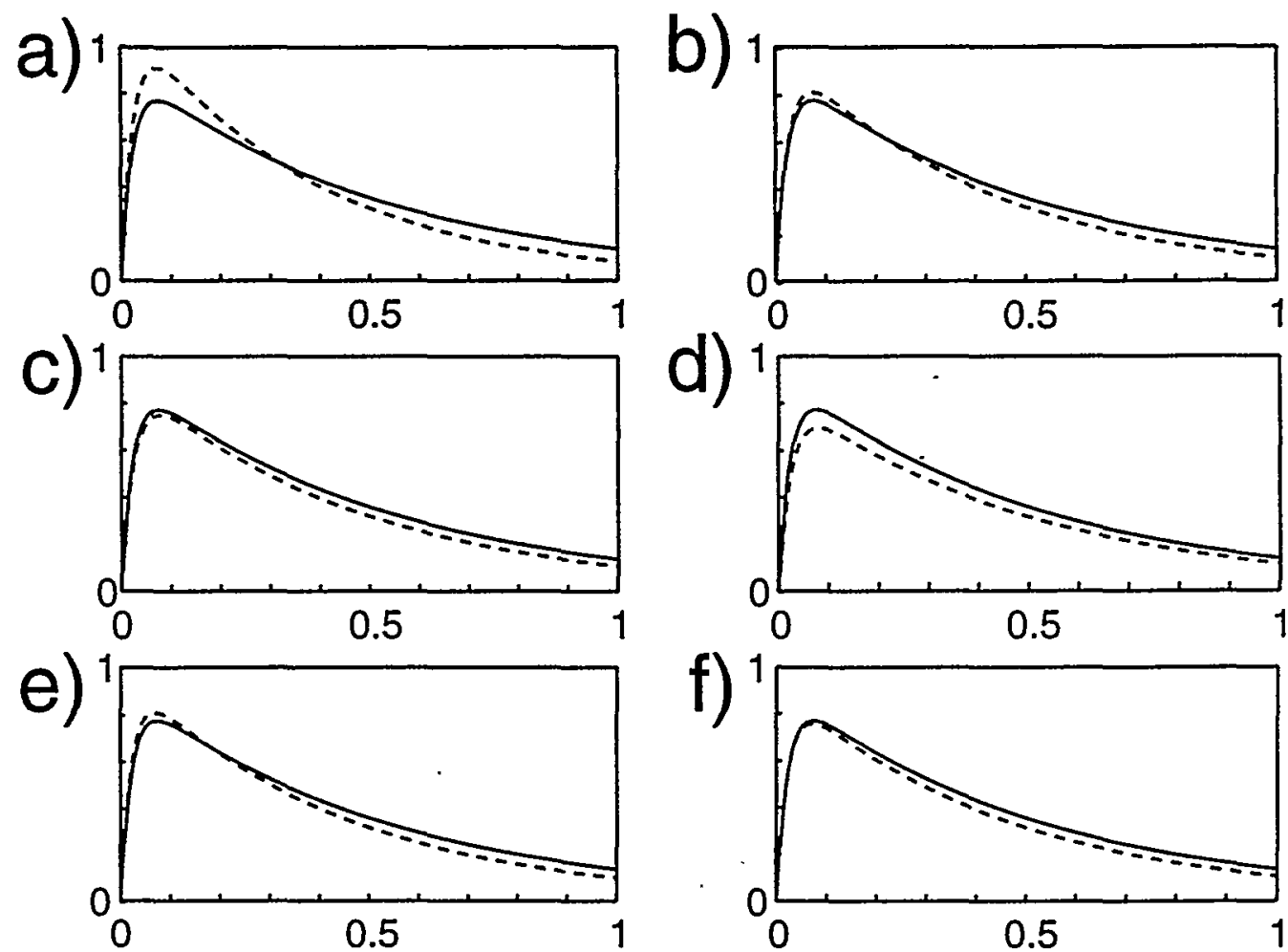

f)
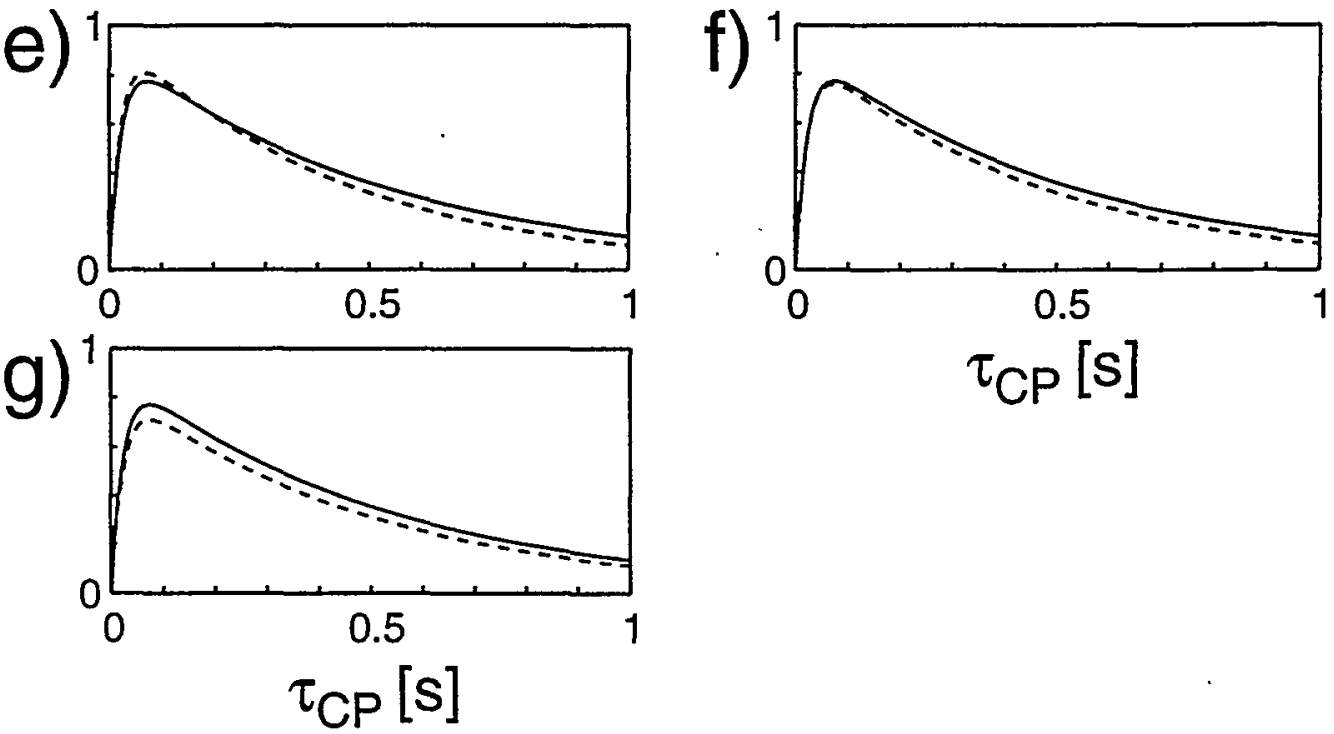

1

\section{$\tau_{\mathrm{CP}}[\mathrm{s}]$}

Figure 4.14 - Simulated cross-polarization time-dependence curves showing the sensitivity of the relative intensities to slight variations in cross-polarization rate constants. In all simulations, $\mathrm{S}_{0}=21$ and $\mathrm{k}_{\mathrm{S}}=36 \mathrm{~s}^{-1}$ (the average of the values in Table 4.1) for both curves. The dotted line represents the cross-polarized silicon intensity for a $\mathrm{Q}^{4}(2 \mathrm{Al})$ site, and the solid line represents the cross-polarized silicon intensity for a $\mathrm{Q}^{4}(1 \mathrm{Al})$ site. The following parameters are held constant in all simulations for the $\mathrm{Q}^{4}(1 \mathrm{Al})$ site (solid line): $\xi=0.83, \mathrm{k}_{\mathrm{I}}=0.08 \mathrm{~s}^{-1}, \mathrm{k}_{\mathrm{SI}}=2.2 \mathrm{~s}^{-1}$, and $\lambda=3$. In all of the simulations for the $\mathrm{Q}^{4}(2 \mathrm{Al})$ site (dotted line), two parameters are held constant: $\xi=0.76$ and $\mathrm{k}_{\mathrm{I}}=0.17 \mathrm{~s}^{-1}$, and the others vary as follows: (a) $\lambda=3 / 2, \mathrm{k}_{\mathrm{SI}}=2.8 \mathrm{~s}^{-1}$; (b) $\lambda=3 / 2$, $\mathrm{k}_{\mathrm{SI}}$ $=2.4 \mathrm{~s}^{-1}$; (c) $\lambda=3 / 2, \mathrm{k}_{\mathrm{SI}}=2.2 \mathrm{~s}^{-1}$; (d) $\lambda=3 / 2, \mathrm{k}_{\mathrm{SI}}=2.0 \mathrm{~s}^{-1}$; (e) $\lambda=2.78, \mathrm{k}_{\mathrm{SI}}=2.6 \mathrm{~s}^{-1}$; (f) $\left.\lambda=2.78, \mathrm{k}_{\mathrm{SI}}=2.4 \mathrm{~s}^{-1} ; \mathrm{g}\right) \lambda=2.78, \mathrm{k}_{\mathrm{SI}}=2.2 \mathrm{~s}^{-1}$ 
Cross-polarization intensities often cannot be quantitatively interpreted even for the relatively simple case of cross polarization between two spin-1/2 nuclei. When quadrupolar nuclei are involved, there is the additional complication that a selective pulse on the central transition will not uniformly excite all possible crystallite orientations. Thus, the value of $\lambda$ for the $Q^{4}(2 \mathrm{Al})$ site would be expected to deviate from $3 / 2$. As discussed in Section 1.2.5, only $9 / 35 \approx 25.7 \%$ of the aluminum spins are expected to be excited by a selective pulse on the central transition. ${ }^{27}$ From simple statistics, one can calculate the probabilities that a given ${ }^{29} \mathrm{Si}$ nucleus in a $\mathrm{Q}^{4}(2 \mathrm{Al})$ site has zero, one, or two excited aluminum neighbors. Since spin-temperature alternation ${ }^{47}$ eliminates all direct (non-cross-polarized) ${ }^{29} \mathrm{Si}$ signal, one only needs to be concerned with the cases of one or two excited aluminum atoms. Taking the weighted average of these two cases gives $\lambda=$ 2.78. While this is significantly different from the value of $\lambda=1.5$ used in the fits above, an examination of the simulations in Figure 4.14e-g reveals that the same general behavior is observed, but the values of $k_{S I}$ are slightly shifted. Since the values of $\lambda$ and $k_{S I}$ are highly correlated, their effects are difficult to deconvolve, and the matter was not pursued further.

It is conceivable to correct for all these unknown scaling factors by explicitly taking the experimental conditions into consideration and simulating the relevant part of the spin system numerically. ${ }^{151}$ Such an approach may enable quantitative extraction of relative intensities from cross-polarization spectra involving half-integer quadrupolar nuclei.

\subsection{Prognosis for Cross-Polarization from Quadrupolar Nuclei Using Low-Rf Field Strengths}

In general, the improvement in signal-to-noise ratio that can be obtained by using cross polarization depends on two factors: (1) the gyromagnetic ratio of the two spins and (2) their longitudinal relaxation times. The relaxation time determines how fast an experiment can be repeated, and a shorter relaxation time has the advantage of permitting faster signal averaging. In low albite, the signal-to-noise ratio per unit time was enhanced by a factor of five for the ${ }^{27} \mathrm{Al}-\mathrm{to}_{-}{ }^{29} \mathrm{Si}$ cross-polarization experiment relative to the directexcitation experiment when both were optimized. The enhancement for the ${ }^{23} \mathrm{Na}$ case was 
a factor of two (see Figure 4.15). Although in a single scan the signal obtained by cross polarization is actually less intense than the direct-excitation signal, there is a still a gain in the signal-to-noise ratio per unit time due to the faster repetition rate in the crosspolarization spectrum ( $5 \mathrm{~s}$ ) compared to the direct-excitation spectrum (2000 s).

The cross-polarization experiment gives less signal per scan than the direct excitation experiment because the cross-polarization efficiency for quadrupolar nuclei is usually very low for a sample spun about an angle greater than $30^{\circ}$ from the static field as a consequence of the time-dependence of the first-order quadrupolar interaction. ${ }^{11,122}$ Although switched-angle spinning experiments take advantage of the increased crosspolarization efficiency for samples spun about an axis parallel to the static magnetic

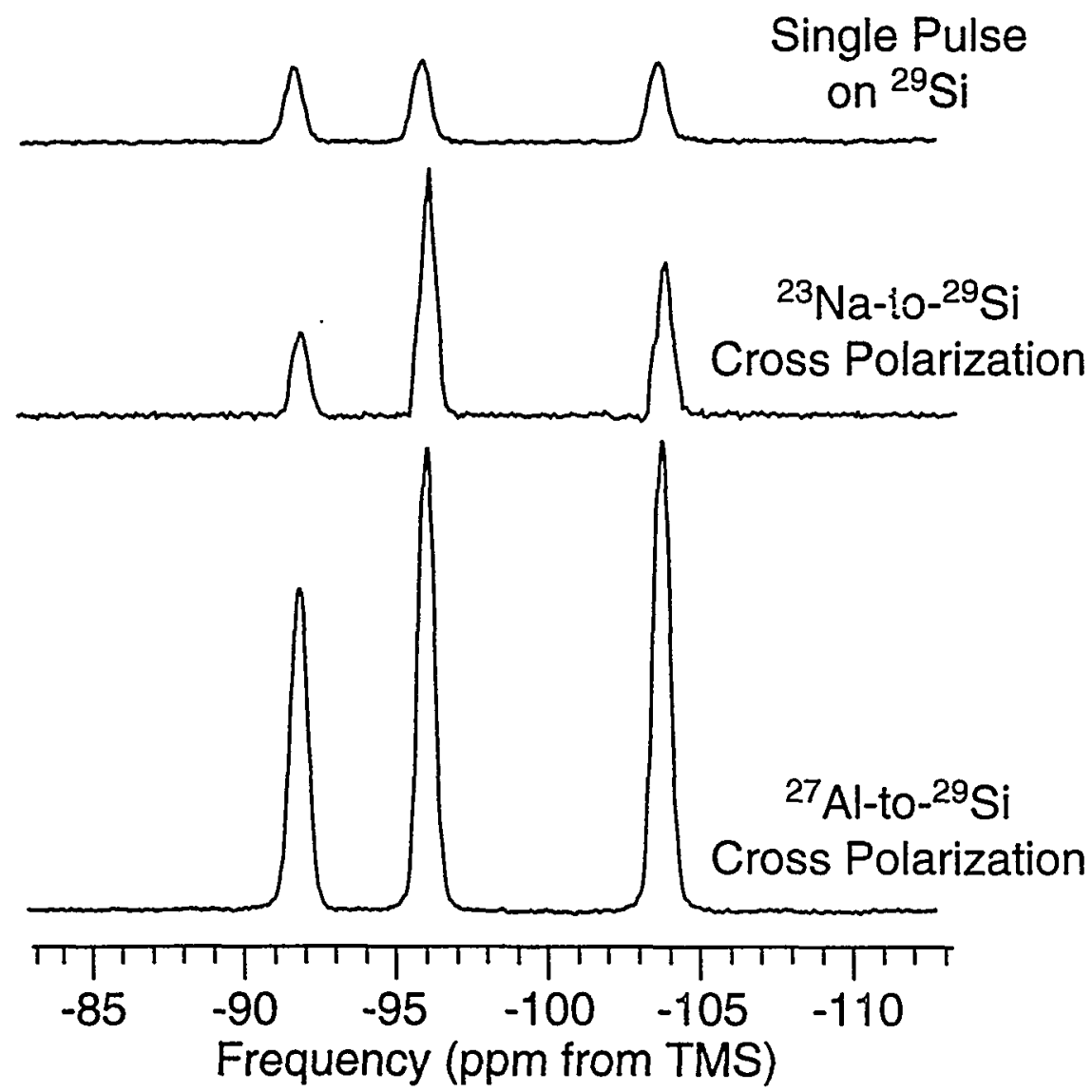

Figure 4.15 - Relative intensities for constant time experiments. All three of these spectra were acquired by signal averaging for $8000 \mathrm{~s}$ although the recycle delays (and, hence, number of scans) differed from experiment to experiment due to differences in $T_{1}$. (The delays were $2000 \mathrm{~s}, 2 \mathrm{~s}$, and $5 \mathrm{~s}$ for the ${ }^{29} \mathrm{Si},{ }^{23} \mathrm{Na}-\mathrm{to}^{29} \mathrm{Si}$, and ${ }^{27} \mathrm{Al}-\mathrm{to}^{29} \mathrm{Si}$ experiments, respectively.) The experiments were normalized by the square root of the number of scans so that the noise levels were the same. 
field, ${ }^{122,144}$ they cannot be performed on conventional MAS equipment. Furthermore, the design of switched-angle spinning probes which are capable of spinning about an axis parallel to the static field ${ }^{155,156}$ precludes the use of a large volume rotor, but use of a large rotor greatly enhances sensitivity. Thus, despite the loss in intensity per scan, cross polarization from quadrupolar nuclei during MAS can still be a useful technique in many cases.

It is possible to enhance the cross-polarized signal further by performing a linearamplitude ramp during the spin lock on the spin-1/2 channel (see Figure 4.16). ${ }^{157,158,159}$ Such a ramp is typically centered at an amplitude equal to the center of one of the match conditions (see Figure 4.5b; in our case, we used the centerband), and the amplitude is stepped over the full width of that condition. The variation in amplitude compensates for dipolar broadening and field inhomogeneity, permitting a larger fraction of the crystallites in the sample to be Hartmann-Hahn matched than in the constant-amplitude case. For sufficiently small ramp slopes, the process can be considered quasi-adiabatic. In principle, equivalent results will be obtained regardless of whether the slope of the ramp is positive or negative or whether the ramp is performed on the $S$ or I channel. Our experiments had to be performed with the ramp on the ${ }^{29} \mathrm{Si}$ side, however, since the spin lock on ${ }^{27} \mathrm{Al}$ was so small. This led to an additional $40 \%$ signal enhancement and a slight modification of the cross-polarization parameters (see Figure 4.17). However, the relative heights of the three peaks did not change. More complicated amplitude-modulation techniques have also been developed, ${ }^{160,161}$ but they require specialized hardware. Furthermore, they are highly optimized for pairs of spin-1/2 nuclei and may not be easily generalizable to quadrupolar systems. For these reasons, they were not pursued further.

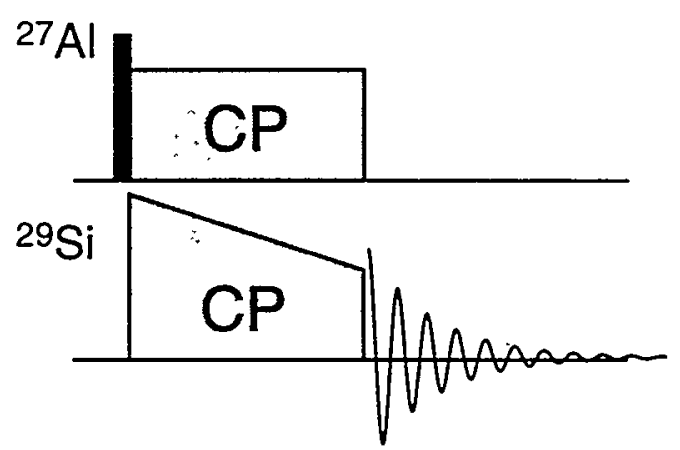

Figure 4.16 - Linear ramp sequence for enhancing cross-polarized signal intensity by matching to all crystallites. 
In heterogenous samples, quantitation can be improved in some cases by performing a chain of cross polarization steps in which the source spins are allowed to
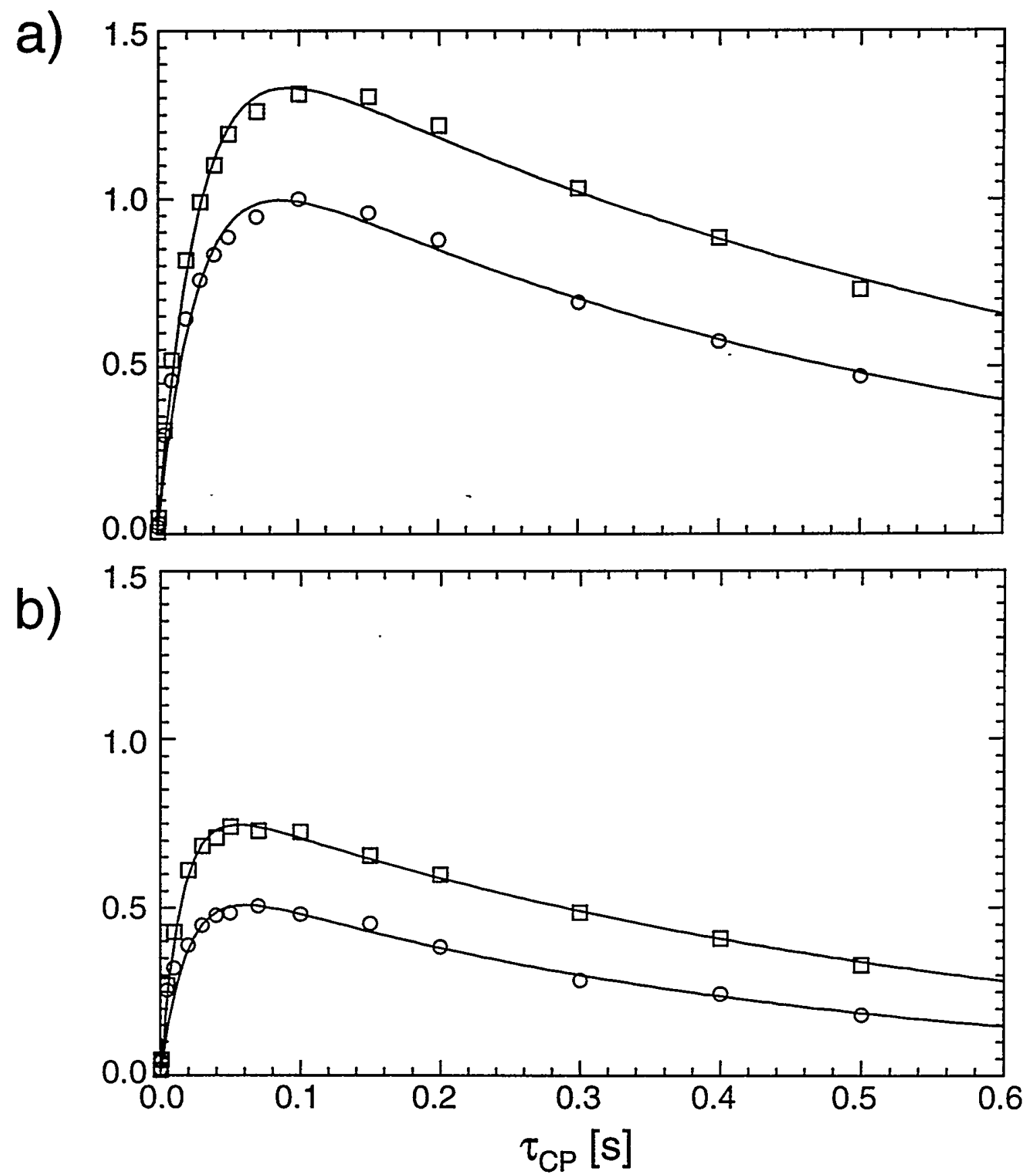

Figure 4.17 - Comparison of cross polarization dynamics for experiments performed using a Hartmann-Hahn match (circles) and linear amplitude ramp (squares). (a) The $\delta=-96.1$ ppm site. (b) The $\delta=-91.8 \mathrm{ppm}$ site. Although intensities were enhanced by $40 \%$ by using the ramp, the CP dynamics were not substantially altered, and the relative intensities of the $\mathrm{Q}^{4}(1 \mathrm{Al})$ and $\mathrm{Q}^{4}(2 \mathrm{Al})$ sites remained the same. 
relax completely between contact periods. ${ }^{162,163}$ Since such a method is not practical for use in two-dimensional experiments, we chose not to explore it with our system.

In addition to large differences in $\mathrm{T}_{1}$ times, two other conditions must be fulfilled for cross polarization from quadrupolar nuclei to be advantageous: (1) the quadrupolar coupling constant must be small enough to allow efficient spin locking, and (2) the $T_{1 \rho}$ relaxation times of both spins must be long compared to the inverse of the cross-relaxation rate constant.
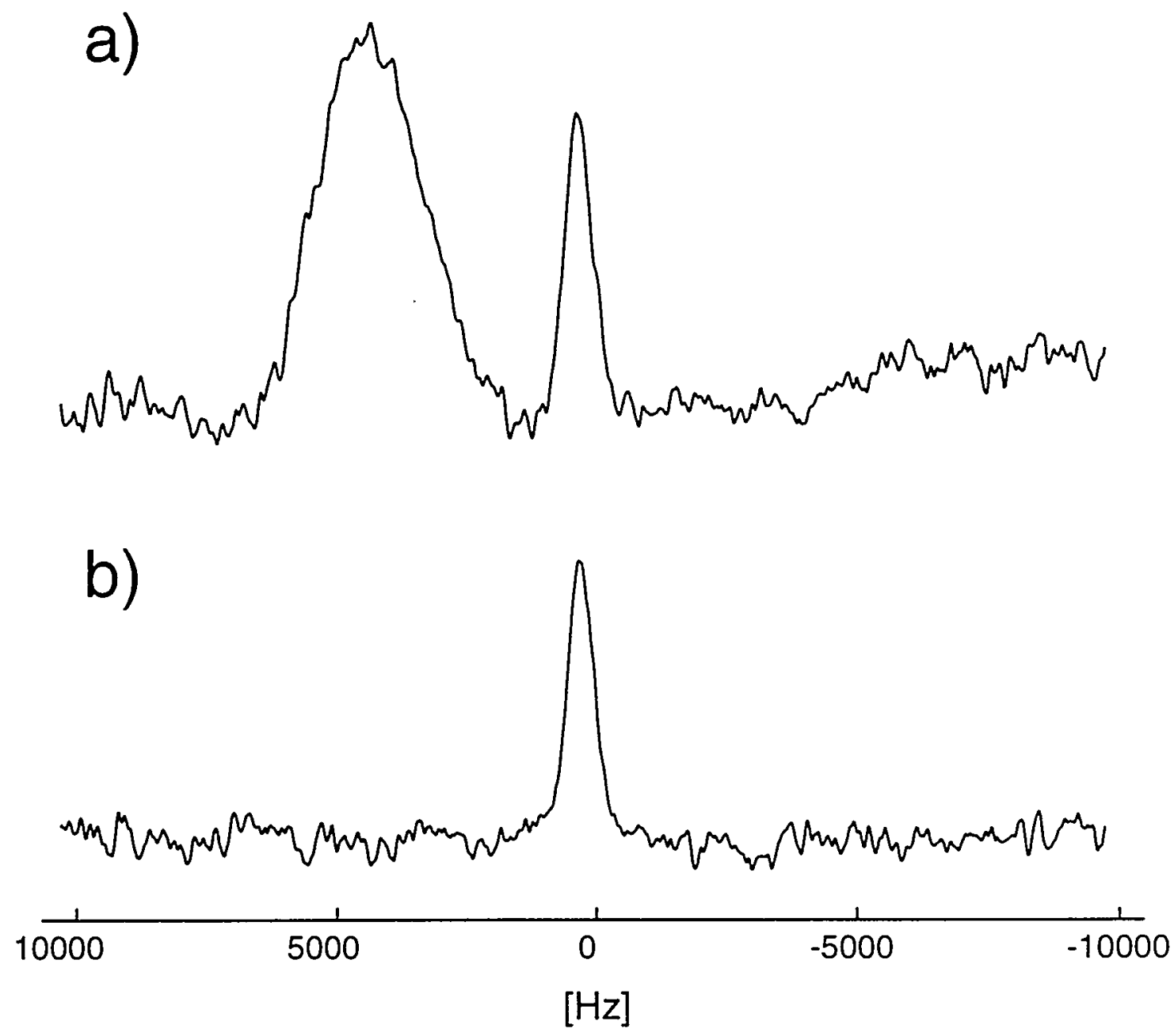

Figure $4.18-{ }^{29} \mathrm{Si}$ spectra of dehydrated zeolite Na-A. (a) Direct ${ }^{29} \mathrm{Si}$ signal. Thirty-two scans were recorded with a recycle delay of $20 \mathrm{~s}$. The broad downfield peak is due to glass ampule in which the sample was sealed. (b) ${ }^{27} \mathrm{Al}-\mathrm{to}^{2}{ }^{29} \mathrm{Si} \mathrm{CP}$ signal. 1056 scans were recorded with a recycle delay of $0.5 \mathrm{~s}$. The spectra are scaled to the same noise level to permit a direct comparison of signal intensities. 
In samples where the spin-1/2 nucleus has a relatively short $T_{1}$, cross polarization from quadrupolar nuclei, although still possible, may not lead to a signal-to-noise enhancement. Figure 4.18 shows two ${ }^{29} \mathrm{Si}$ spectra of dehydrated zeolite Na-A, both acquired with 10.7 minutes of signal averaging. The experimental parameters were similar to those described in Section 4.2. The spectra are scaled to the same noise level, allowing a direct comparison of signal intensities. Although the cross-polarized spectrum eliminates the background ${ }^{29} \mathrm{Si}$ signal from the glass ampule, the signal-to-noise per unit time is approximately the same in both cases. However, the presence of a cross-polarized signal can be useful in heteronuclear correlation experiments even if it does not lead to signal enhancement (as will be demonstrated in Chapter 6). 


\section{Chapter 5: Applications of Cross Polarization from Quadrupolar Nuclei to Aluminosilicates}

An advantage of the faster repetition rate for ${ }^{27} \mathrm{Al}-$ to- ${ }^{29} \mathrm{Si}$ cross polarization is the increased feasibility of two-dimensional experiments since a five-fold increase in signal enhancement corresponds to a twenty-five fold savings in time. In this chapter and the next, we present two-dimensional experiments which demonstrate applications of cross polarization from quadrupolar nuclei.

\subsection{Isotropic-Anisotropic Correlaticn Spectroscopy}

Due to its typical high resolution and sensitivity to local atomic environments, solid-state ${ }^{29} \mathrm{Si}$ NMR has been used extensively to study a wide range of materials and

materials-related problems. Most of the chemical information obtained using ${ }^{29} \mathrm{Si} \mathrm{NMR}$ has utilized the isotropic chemical shift. Through empirical relationships, information about aluminum occupancy of next-nearest neighbor positions, Si-O-T bond angles, and $\mathrm{Si}-\mathrm{O}$ bond lengths have been obtained. ${ }^{110.164}$

The chemical shift is strongly dependent on the local electronic environment and on the orientation of a crystallite relative to the static magnetic field. To fully characterize the local acomic environment, both the isotropic and the anisotropic components of the chemical shift need to be determined. For single crystals the complete orientationdependent chemical-shielding tensor can be determined by measuring NMR spectra as a function of orientation of the crystal about three axes relative to the magnetic field ${ }^{6}$ or by a more efficient technique that involves the sudden reorientation of the crystal during a twodimensional NMR experiment. ${ }^{165,166,167}$ For powders, the chemical-shielding interaction can be determined from NMR spectra of a static or slowly rotating sample. When multiple atomic sites are present, however, the powder patterns will often overlap, precluding the determination of the chemical-shielding parameters from a simple onedimensional spectrum.

The problem of spectral overlap can be overcome by the use of two-dimensional NMR techniques which combine the high resolution of an isotropic chemical-shift spectrum in one dimension with the high information content but low resolution of an 
anisotropic spectrum in a second dimension. Two-dimensional isotropic-anisotropic correlation techniques have led to the greater utilization of the anisotropic chemical shift for the characterization of the local atomic environment, for the study of molecular motion, and for comparisons with theoretical calculations. ${ }^{23}$

Recently, many isotropic-anisotropic correlation techniques have been developed including: Magic-Angle Hopping (MAH), ${ }^{168}$ Magic-ringle Turning (MAT), TOSSreTOSS, ${ }^{169}$ Switched-Angle Spinning (SAS), ${ }^{170,171,172}$ Variable-Angle Correlation SpectroscopY (VACSY), ${ }^{90,173}$ experiments involving changes in the spinning speed ${ }^{174,175}$ and variations of the above. All of these techniques involve reorientation of the powder sample either by hops between discrete positions (MAH), by sample spinning (MAT, TOSS-reTOSS, and the variable speed techniques), or by a combination of the two (VACSY, SAS). VACSY, SAS and the variable speed techniques will not be discussed further in this thesis since they cannot be carried out with a conventional MAS set-up. The Magic-Angle Hopping experiment also requires special equipment but will briefly be mentioned because it is the conceptual predecessor of Magic-Angle Turning.

\subsection{Magic-Angle Hopping}

Magic-Angle Hopping (MAH) ${ }^{168,176}$ was one of the first techniques developed to produce isotropic-anisotropic correlation spectra. The experiment makes use of the fact that anisotropic second-rank interactions will be averaged to zero under transformations that have cubic (or higher) symmetry. ${ }^{177}$ Rapid magic-angle spinning is one example of such a transformation, but it can be shown ${ }^{11}$ that three discrete $120^{\circ}$ "hops" about the magic-angle axis also suffice to average out the chemical-shielding anisotropy. A derivation of this property as it applies to the MAH experiment will not be presented here, but the same principle will be discussed in more detail in Section 5.3.1 in relation to MAT.

Figure 5.1 shows the Magic-Angle Hopping pulse sequence (with the conventional use of the label $S$ for the directly detected spins). The evolution period is divided into three segments, each of which occurs at a different rotor phase angle. Z-filters ${ }^{4}$ are used to store one component of the magnetization along the static field while the sample is 
reoriented. At the end of $t_{1}$, only the isotropic part of the chemical shift remains. Twodimensional Fourier transformation leads to a spectrum in which isotropic shifts are correlated with static powder patterns.

While the MAH experiment is conceptually elegant, it requires a specialized probe design ${ }^{176}$ which, although commercially available, is not found in many NMR laboratories. Another drawback of the experiment is that static powder patterns have poor signal-to-noise compared to MAS sideband patterns. Both of these problems can be circumvented by performing slow-spinning variations of the experiment.

\subsection{Isotropic-Anisotropic Correlation by Slow Spinning}

The MAH pulse sequence of Figure 5.1 can, in fact, be applied to rotating samples as was first shown by Gan. ${ }^{178}$ In this experiment, the pulses are synchronized with the sample rotation so that $t_{1}$ evolution occurs after the sample has rotated by $120^{\circ}$ increments. In the limit that $t_{1}$ is much smaller than the rotor period (requiring rotation rates of less than $100 \mathrm{~Hz}$ ), it is possible to view the slow-spinning experiment as an approximation to the discrete hopping experiment.

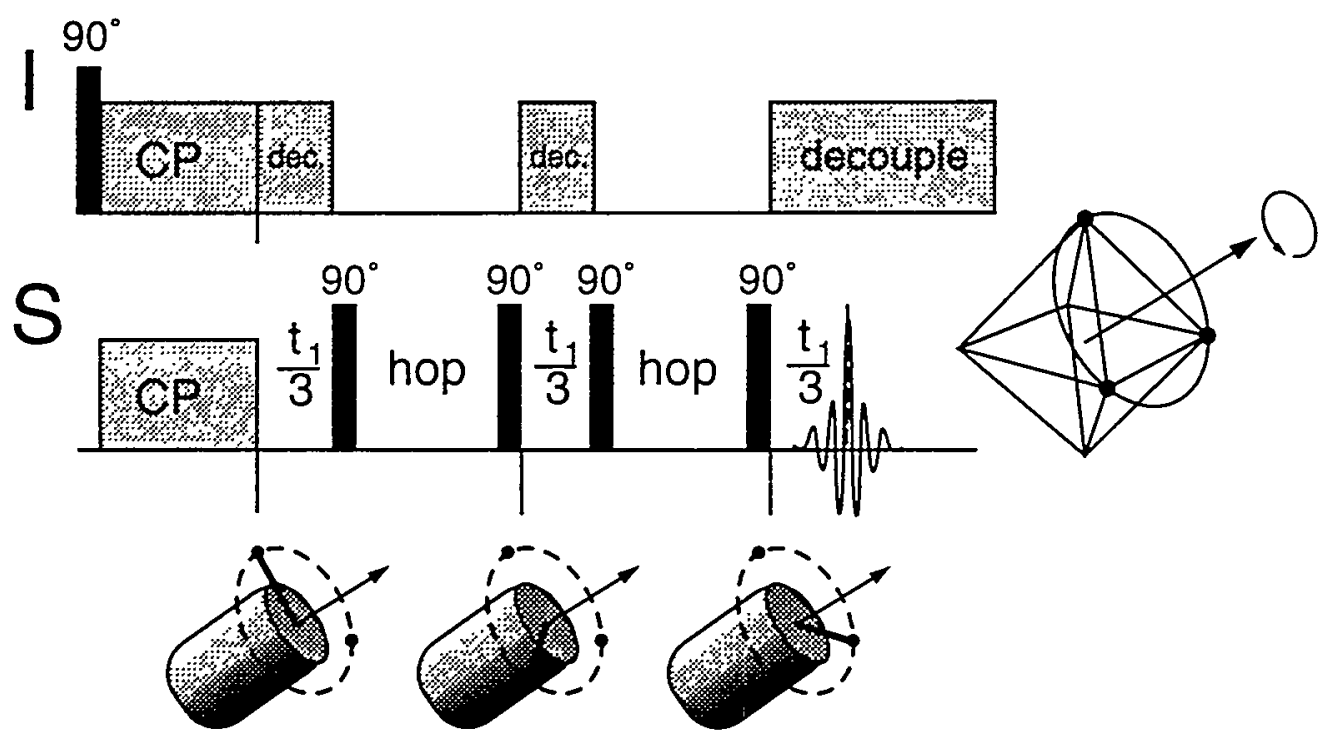

Figure 5.1 - The Magic-Angle Hopping experiment. The sample is static during each portion of the evolution period. Z-filters are used to store a component of the magnetization as the sample is reoriented by $120^{\circ}$ about the magic-angle axis. These positions correspond to three vertices of an octahedron. At the end of the $t_{1}$ period, the chemical shift anisotropy has been averaged out. 
Using such a slow spinning speed is not the best way to perform the experiment, however, since it leads to distorted powder patterns which are difficult to interpret. A better approach is to spin at a fast enough rate that distinct spinning sidebands appear in the anisotropic dimension. This will improve the signal-to-noise and, hence, the reliability of fits of the CSA parameters. In addition, when the S-spin chemical-shielding anisotropy is greater than the S-spin homonuclear dipolar coupling (as is the case for nuclei with low gyromagnetic ratios and low natural abundance such as ${ }^{13} \mathrm{C}$ or ${ }^{29} \mathrm{Si}$ ), it will be possible to choose a spinning rate that makes the sideband intensities independent of the dipolar interaction. To theoretically describe the case where the evolution period is a significant fraction of the rotor period, one must consider the time-dependence explicitly"l as will be shown below (Section 5.3.1).

The pulse sequence of Figure 5.1 is not the only possible sequence that can be used to implement this concept. Variants using $180^{\circ}$ pulses hâve been developed by Gann et al. ${ }^{179}$ and Hu et al. ${ }^{180}$ Collectively, these experiments are known as Magic-Angle Turning experiments, ${ }^{11,180}$ and they will be discussed in detail in the following sections.

\subsubsection{Theory of Magic-Angle Turning Experiments}

To show how Magic-Angle Hopping and Magic-Angle Turning experiments lead to an isotropic spectrum in the $\omega_{1}$ dimension, the theory behind such experiments will be outlined. Related derivations can also be seen in the Ph.D. theses of Baltisberger ${ }^{11}$ and Gann. ${ }^{181}$

Figure 5.2 shows a schematic of a generalized MAH/MAT type of experiment. It is not meant to represent a particular pulse sequence but will serve as a framework for the following discussion. The periods labeled $\delta$ will be constructively added in several of the pulse sequences to form the evolution period, $\mathrm{t}_{1}$. (In one case, ${ }^{180}$ the evolution period is formed slightly differently, but the same formalism can still be used.) $\Gamma$ represents the number of basic building blocks (groups of pulses) used to construct the sequence, and $\mathrm{N}$ and $\mathrm{k}$ are integers. The isotropic echo is formed at the time $\tau^{\prime}$. In the following derivation, we will assume that the pulses are short enough relative to the rotor period that their finite width can be neglected. 
During an MAT experiment, the magnetization evolves under the secular chemical-shift Hamiltonian (see Section 1.2.3)

$$
\mathcal{H}_{\mathrm{CS}}=\Omega(\mathrm{t}) \mathrm{I}_{\mathrm{z}}
$$

where the time-dependent frequency of the chemical shift for a single crystallite is given by (see Equations (1.108) and (1.60))

$$
\Omega(t)=\sum_{1} C_{1} \sum_{m=-1}^{1} D_{m, 0}^{(1)}\left(\omega_{r} t+\phi_{r}, \theta, 0\right) A_{1, m}^{c s} .
$$

The $\left\{C_{1}\right\}$ are constants, and $A_{1, m}^{C S}$ defines the orientation of the chemical shift principal values relative to the rotor axis (via the Euler angles $\alpha^{C S}, \beta^{C S}$, and $\gamma^{C S}$ ). In the zerothorder average Hamiltonian approximation, the phase acquired during the evolution period Of an MAT pulse sequence can be determined by integrating Equation (5.2) over time and making use of the fact that the pulse sequence contains repetitive building blocks

$$
\begin{gathered}
\Phi\left(\tau^{\prime}\right)=\sum_{j=0}^{\Gamma-1}\left[J \int_{N \tau_{r} j / \kappa}^{\left(N \tau_{r} j / \kappa\right)+\delta} \Omega(t) d t+K \int_{\left(N \tau_{r} j / \kappa\right)+\delta}^{N \tau_{r}(j+1) / \kappa} \Omega(t) d t\right] \\
+L \int_{\Gamma N \tau_{r} / \kappa}^{\tau^{\prime}} \Omega(t) d t .
\end{gathered}
$$

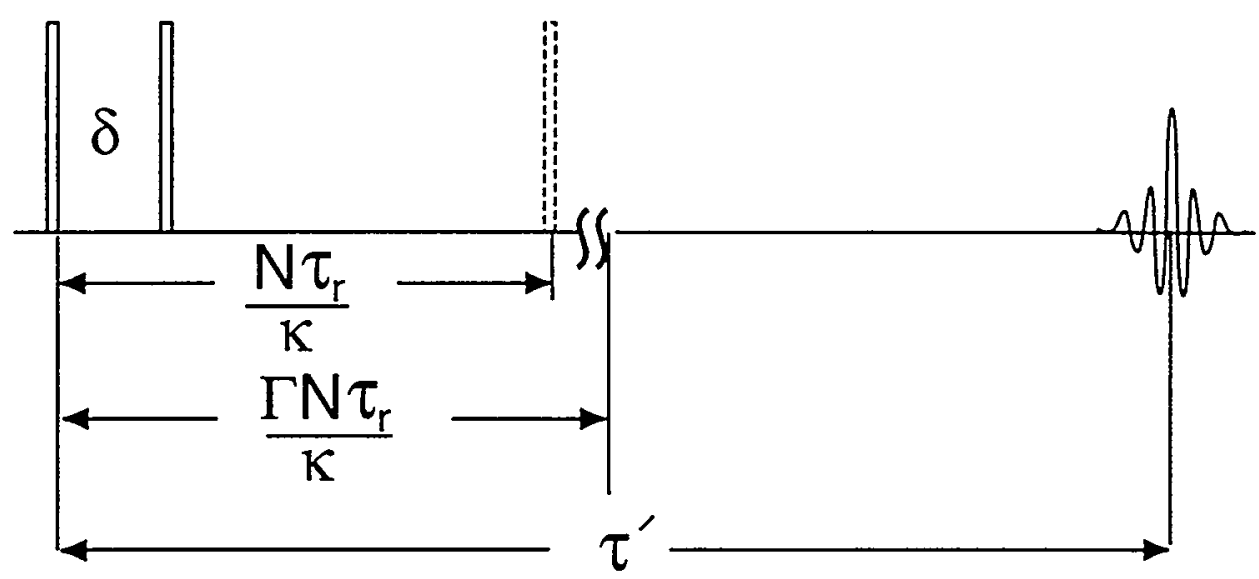

Figure 5.2 - Generalized MAH/MAT type experiment. The basic building block is repeated $\Gamma$ times, and the periods $\delta$ contribute to the evolution. $N$ and $\kappa$ are integers; restrictions on their values are described in the text. 
Here, the terms $\mathrm{J}, \mathrm{K}$, and $\mathrm{L}$ have been introduced to describe the effect of the pulses on the spins since the types of pulses that are applied dictate how the phase accumulates in the transverse plane (e.g. - a $180^{\circ}$ pulse reverses the sign of the phase of a given crystallite ${ }^{182}$ ). Thus, Equation (5.3) represents the combined effects of the pulse sequence on both spin space and geometrical space.

Substituting Equations (5.2) and (1.36) into Equation (5.3) and factoring out the $\mathrm{m}=0$ term gives

$$
\begin{aligned}
& \Phi\left(\tau^{\prime}\right)=\sum_{1} C_{1} d_{0,0}^{(1)}(\theta) A_{1,0}^{C S}\left\{\sum_{j=0}^{\Gamma-1}\left[J \int_{N \tau_{r} j / x}^{\left(N \tau_{r} j / k\right)+\delta} d t+K \int_{-\left(N \tau_{r} j / x\right)+\delta}^{N \tau_{r}(j+1) / \kappa} d t\right]\right. \\
& \left.+L \int_{\Gamma \mathrm{N} \tau_{\mathrm{r}} / \kappa}^{\tau^{\prime}} \mathrm{dt}\right\}+\sum_{\mathrm{l}} \mathrm{C}_{\mathrm{l}} \sum_{\mathrm{m}=-\mathrm{l} ; \mathrm{m} \neq 0}^{1} \mathrm{e}^{-\mathrm{im} \phi_{\mathrm{r}}(1)} \mathrm{m}, 0^{(1)}(\theta) \mathrm{A}_{\mathrm{l}, \mathrm{m}}^{\mathrm{CS}} \\
& \times\left\{\sum_{j=0}^{\Gamma-1}\left[J \int_{N \tau_{r} j / \kappa}^{\left(N \tau_{r} j / \kappa\right)+\delta} e^{-i m \omega_{r} t} d t+K \int_{\left(N \tau_{r} j / \kappa\right)+\delta}^{N \tau_{r}(j+1) / \kappa} e^{-i m \omega_{r} t} d t\right]\right. \\
& \left.+\mathrm{L} \int_{\Gamma \mathrm{N} \tau_{\mathrm{r}} / \mathrm{K}}^{\tau^{\prime}} \mathrm{e}^{-\mathrm{im} \omega_{\mathrm{r}} \mathrm{t}} \mathrm{dt}\right\}
\end{aligned}
$$

Integrating Equation (5.4) and using the fact that $\omega_{\mathrm{r}} \tau_{\mathrm{r}}=2 \pi$ leads to

$$
\begin{aligned}
& \Phi\left(\tau^{\prime}\right)=\sum_{\mathrm{l}} \mathrm{C}_{\mathrm{l}} \mathrm{d}_{0,0}^{(1)}(\theta) \mathrm{A}_{\mathrm{l}, 0}^{\mathrm{CS}}\left\{\mathrm{L}\left(\tau^{\prime}-\frac{\Gamma \mathrm{N} \tau_{\mathrm{r}}}{\mathrm{K}}\right)+\sum_{\mathrm{j}=0}^{\Gamma-1}\left[\mathrm{~J} \delta+\mathrm{K}\left(\frac{\mathrm{N} \tau_{\mathrm{r}}}{\mathrm{K}}-\delta\right)\right]\right\} \\
& +\sum_{l} C_{l} \sum_{m=-l ; m \neq 0}^{1} e^{-i m \phi_{r}} d_{m, 0}^{(l)}(\theta) A_{l, m}^{C S}\left(\frac{i}{m \omega_{r}}\right) \\
& \times\left\{\left[(J-K) e^{-i m \omega_{r} \delta}-J+K e^{-(i m N 2 \pi) / \kappa}\right] \sum_{j=0}^{\Gamma-1}\left(e^{-i m N 2 \pi} \frac{j}{k}\right)^{j}\right. \\
& \left.+\mathrm{L}\left(\mathrm{e}^{-\mathrm{im} \omega_{\mathrm{r}} \tau^{\prime}}-\mathrm{e}^{-(\mathrm{im} \Gamma \mathrm{N} 2 \pi) / \mathrm{k}}\right)\right\} \text {. }
\end{aligned}
$$

For the chemical-shielding interaction, 1 can only have the values 0 or 2. Under MAS conditions, $\mathrm{d}_{0,0}^{(2)}$ equals zero, and the $\mathrm{m}=0$ term in Equation (5.5) simplifies to 


$$
\mathrm{C}_{0} \sigma_{i s o}\left\{\Gamma \delta(\mathrm{J}-\mathrm{K})+\frac{\mathrm{K} \Gamma \mathrm{N} \tau_{\mathrm{r}}}{\kappa}+\mathrm{L}\left(\tau^{\prime}-\frac{\Gamma N \tau_{\mathrm{r}}}{\kappa}\right)\right\}
$$

where the summation over $\mathrm{j}$ has been performed.

The remaining terms in Equation (5.5) contain the anisotropic parts of the chemical-shielding interaction. To see how these terms can be made to vanish, it is useful to perform the sum over $j$ explicitly. Since it is a geometric series, it can be analytically evaluated

$$
\sum_{j=0}^{\Gamma-1}\left(e^{-\frac{i m N 2 \pi}{\kappa}}\right)^{j}=\frac{1-e^{-(i m \Gamma N 2 \pi) / \kappa}}{1-e^{-(i m N 2 \pi) / \kappa}}
$$

The denominator cannot equal zero which means that

$$
\frac{\mathrm{mN}}{\mathrm{K}} \neq \text { integer } ; \mathrm{m}= \pm 1, \pm 2
$$

The lowest value of $\kappa$ for which this relation can be satisfied is $\kappa=3$; then it will hold for all values of $\mathrm{N}$ that are not multiples of 3 . This constrains the number of rotor cycles over which an MAT-type experiment can be performed. All MAT sequences published to date use $\kappa=3$. Note that similar principles have been used to remove spinning sidebands for odd-half-integer quadrupolar nuclei although in that case five $72^{\circ}$ rotations about the angle $63.43^{\circ}$ are necessary. ${ }^{179}$

It is now necessary to consider specific details of the pulse sequences to demonstrate how the different flavors of MAT produce isotropic spectra in $\omega_{1}$. For the experiment of $\mathrm{Gan}^{178}$ (see Figure 5.3), the $90^{\circ}$ pulses act as z-filters. Therefore, evolution in the transverse plane will only occur during the periods $\delta$ and during the acquisition. This corresponds to the case where $\mathrm{J}=1, \mathrm{~K}=0$, and $\mathrm{L}=1$; thus, Equation (5.6) becomes

$$
\mathrm{C}_{0} \sigma_{\mathrm{iso}}\left\{\Gamma \delta+\left(\tau^{\prime}-\frac{\Gamma N \tau_{\mathrm{r}}}{\kappa}\right)\right\}
$$

and the anisotropic term is given by 


$$
\frac{\left(\mathrm{e}^{-\mathrm{im} \omega_{\boldsymbol{r}} \delta}-1\right)\left(1-\mathrm{e}^{-(\mathrm{im} \Gamma \mathrm{N} 2 \pi) / \mathrm{k}}\right)+\left(\mathrm{e}^{-\mathrm{im} \omega_{r} \tau^{\prime}}-\mathrm{e}^{-(\mathrm{im} \Gamma \mathrm{N} 2 \pi) / \mathrm{k}}\right)\left(1-\mathrm{e}^{-(\mathrm{imN} 2 \pi) / \mathrm{k}}\right)}{\left(1-\mathrm{e}^{-(\mathrm{imN} 2 \pi) / \mathrm{k}}\right)}
$$

In the sequence of Figure 5.3, two blocks of pulses are applied $(\Gamma=2)$. When the time interval between the end of the last block and the start of acquisition is set equal to $\delta$, one can show (after a lot of algebra) that the numerator in Equation (5.10) is zero. Thus, the anisotropy is eliminated, and a purely isotropic echo is formed. Furthermore, it is easy to see that Equation (5.9) reduces to $C_{0} \sigma_{\text {iso }}\left\{3 \delta_{r}\right\}$ so $\delta$ must equal $t_{1} / 3$.

In the experiments of Gann et al. ${ }^{179}$, evolution occurs in the transverse plane throughout the entire pulse sequence. The $180^{\circ}$ pulses will simply change the sign of the chemical-shift Hamiltonian, corresponding to $\mathrm{J}=1, \mathrm{~K}=-1$, and $\mathrm{L}=1$. Equation (5.6), therefore, becomes

$$
\mathrm{C}_{0} \sigma_{\mathrm{iso}}\left\{2 \Gamma \delta-\frac{\Gamma N \tau_{\mathrm{r}}}{\kappa}+\left(\tau^{\prime}-\frac{\Gamma N \tau_{\mathrm{r}}}{\kappa}\right)\right\}
$$

From Figure 5.5, we see that $\Gamma=\kappa=3$. This means that the sum in Equation (5.7) equals zero and the anisotropic term has the form

$$
\left(e^{-i m \omega_{r} \tau^{\prime}}-e^{-i m N 2 \pi}\right)
$$

which vanishes whenever $\tau^{\prime}$ is an integer multiple of the rotor period. Thus, a series of isotropic echoes spaced at integer multiples of the rotor period will be recorded during the acquisition period. ${ }^{183}$ These are the well-known MAS rotational echoes, and when Fourier-transformed they lead to sidebands in the frequency domain. ${ }^{39}$ The spin echo (due to the $180^{\circ}$ pulses) will occur when $\tau^{\prime}=2 \mathrm{~N} \tau_{\mathrm{r}}$ (see Equation (5.11)) so $\delta$ is $\mathrm{t}_{1} / 6$.

The experiments of $\mathrm{Hu}$ et al. ${ }^{180}$ need to be described somewhat differently because of their unconventional evolution scheme. Still, the above formalism can be used if $\delta$ is replaced by $\left(N \tau_{r} / 6+\varepsilon\right)$ for the positive evolution experiment (see Figure 5.7). The accumulation of phase is described by $\mathrm{J}=1, \mathrm{~K}=-1, \mathrm{~L}=-1$, and Equation (5.6) becomes

$$
\mathrm{C}_{0} \sigma_{\text {iso }}\left\{6\left(\frac{N \tau_{\tau}}{6}+\varepsilon\right)-\frac{\Gamma N \tau_{\tau}}{\kappa}-\left(\tau^{\prime}-\frac{\Gamma N \tau_{\tau}}{\kappa}\right)\right\}
$$


Since $\Gamma=\kappa=3$, the anisotropic terms again vanish whenever $\tau^{\prime}$ is an integer number of rotor periods. The spin echo will be formed at $\tau^{\prime}=N \tau_{\tau}$, and, therefore, and $\varepsilon=t_{1} / 6$.

Clearly, isotropic echoes can be formed by all three pulse sequences. In the next two sections we will examine experimental aspects of the techniques and compare their performance as applied to low albite.

\subsubsection{MAT with $90^{\circ}$ Pulses}

Figure 5.3 shows the first version of the MAT experiment proposed by $\operatorname{Gan}^{178}$ in 1992. The pulse sequence is identical to the MAH sequence of Figure 5.1 except that the sample is continuously rotated rather than discretely jumped. While the sequence successfully eliminates the anisotropy in the $\omega_{1}$ dimension (as demonstrated in the previous section), it cannot be used to generate pure absorption-mode lineshapes in that dimension, despite assertions to the contrary. ${ }^{11,178}$ (The earlier MAH paper ${ }^{168}$ did not claim to be phase-sensitive, and spectra were displayed in the absolute value mode.) The reason that pure-phase spectra cannot be obtained can be seen by inspecting the pulse sequence and coherence-transfer pathway shown in Figure 5.3. The $90^{\circ}$ pulse after the second zfilter restores both +1 and -1 quantum coherences, but the detector records only one of

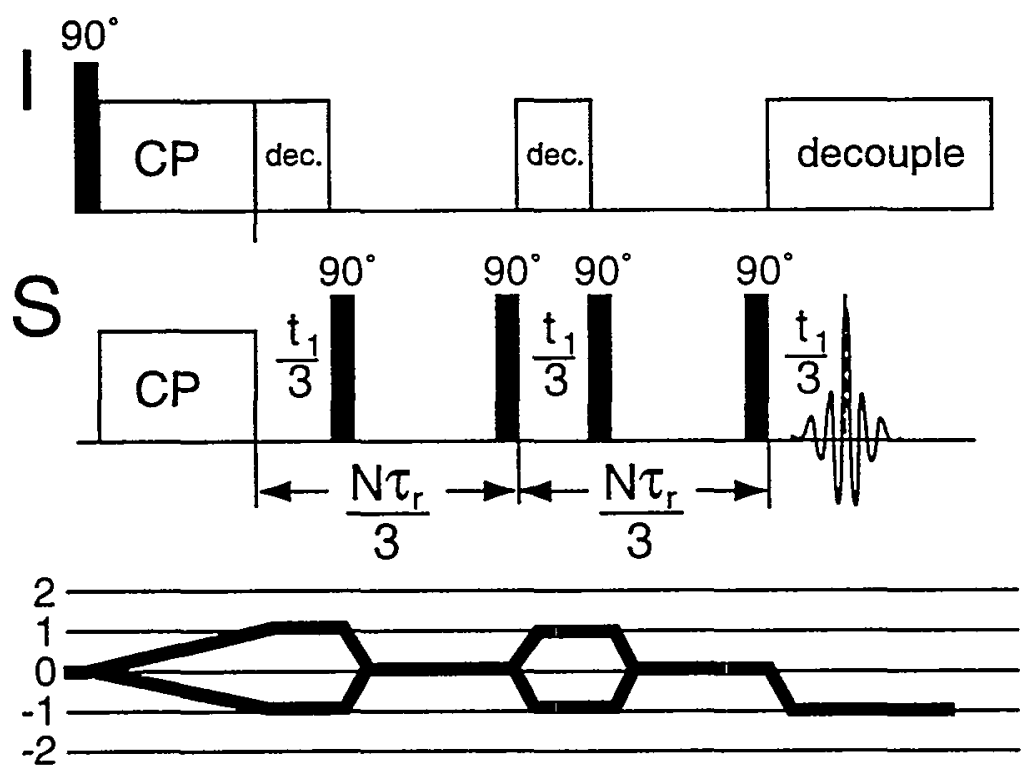

Figure 5.3 - The original MAT experiment ${ }^{178}$. Note that the pulse sequence fails to retain both the +1 and -1 coherences throughout the entire evolution period, preventing pure absorption-phase spectra from being recorded. 
them (the -1 quantum coherence in this figure). However, no pulse separates the last portion of the $t_{1}$ period from the acquisition period, $t_{2}$. Thus, the evolution of the +1 quantum coherence during the last part of the $t_{1}$ period is not recorded, prohibiting phasesensitive detection in $\omega_{1}{ }^{4}$ Simply applying whole-echo acquisition to the sequence with only $90^{\circ}$ pulses as has been suggested ${ }^{11}$ does not alleviate this problem since a whole echo cannot be obtained for small values of $t_{1}$. It is possible to add a $180^{\circ}$ pulse after the last $90^{\circ}$, however, ${ }^{184}$ in a combined hypercomplex/whole- $\epsilon$ ino acquisition experiment (see Figure 1.7d). This not only produces pure-absorption lineshapes but also can eliminate probe-ringdown effects. ${ }^{185}$ Variations which incorporate echoes in each segment of the $t_{1}$ period to suppress artifacts due to relaxation also exist. ${ }^{185,186}$

It is, however, possible to obtain a pure-phase, isotropic-anisotropic spectrum using only $90^{\circ}$ pulses. A sequence that does this was also proposed by $\mathrm{Gan}^{183}$ and is depicted in Figure 5.4. Like the original MAT experiment, the symmetry of $120^{\circ}$ rotations about the magic-angle axis is exploited, but the definition of $t_{1}$ used in this experiment is somewhat unconventional. By varying both the time at which signal acquisition is begun and the phase cycling of the receiver, experiments with effectively different signs of $t_{1}$ can be recorded as described elsewhere. ${ }^{183}$ The experiment labeled $\mathrm{P}^{+}$in Figure 5.4 corresponds to a $\mathrm{t}_{1}$-domain signal proportional to $\mathrm{e}^{\mathrm{iC}_{0} \sigma_{\mathrm{iso}}\left\{3\left(\mathrm{t}_{1} / 2\right)\right\}}$ (neglecting relaxation) while the experiment labeled $\mathrm{P}^{-}$corresponds to $\mathrm{e}^{\mathrm{iC}_{0} \sigma_{\mathrm{tso}}\left\{3\left(-\mathrm{t}_{1} / 2\right)\right\}}$. To process the data, one must first apodize the $\mathrm{P}^{+}$and $\mathrm{P}^{-}$data sets separately since the echo maxima shift in opposite directions in increments of half the $t_{1}$ dwell time. Hypercomplex cosine and sine data sets can be then formed by linear combination of these two data sets, and Fourier. transformation in both dimensions gives a skewed spectrum. It is tempting to think that one should shear the spectrum by using Equation (1.141) with $f=1 / 3$ to obtain more easily interpretable results. However, the rotational echoes present in the $t_{2}$ dimension of the $\mathrm{P}^{+}$ and $\mathrm{P}^{-}$data sets lead to sidebands in the two-dimensional spectrum, and shearing would produce anisotropic shapes that are difficult to interpret. ${ }^{183}$

The pulse sequence of Figure 5.4 was applied to low albite, but the quality of the recorded spectrum was poor (data not shown) with dispersive contributions to the lineshape even in the unsheared spectrum. A possible reason for this is the combination of a long ${ }^{29} \mathrm{Si} \mathrm{T}_{2}$ relaxation time and weak heteronuclear couplings in low albite. The pulse 
sequences of Figures 5.1, 5.3, and 5.4 were designed under the assumption that the magnetization which remains in the transverse plane after the first $90^{\circ}$ pulse of the $z$-filter will completely dephase before the second $90^{\circ}$ pulse..$^{186}$ While this is a good approximation for ${ }^{13} \mathrm{C}$ spins coupled to many protons, ${ }^{168}$ it may not be valid for our case where the heteronuclear couplings are weak and transverse relaxation slow. Although, in principle, it would be possible to create a longer phase cycle that eliminates the effect of this residual magnetization, there is a better alternative for samples with long $T_{2}$ times.
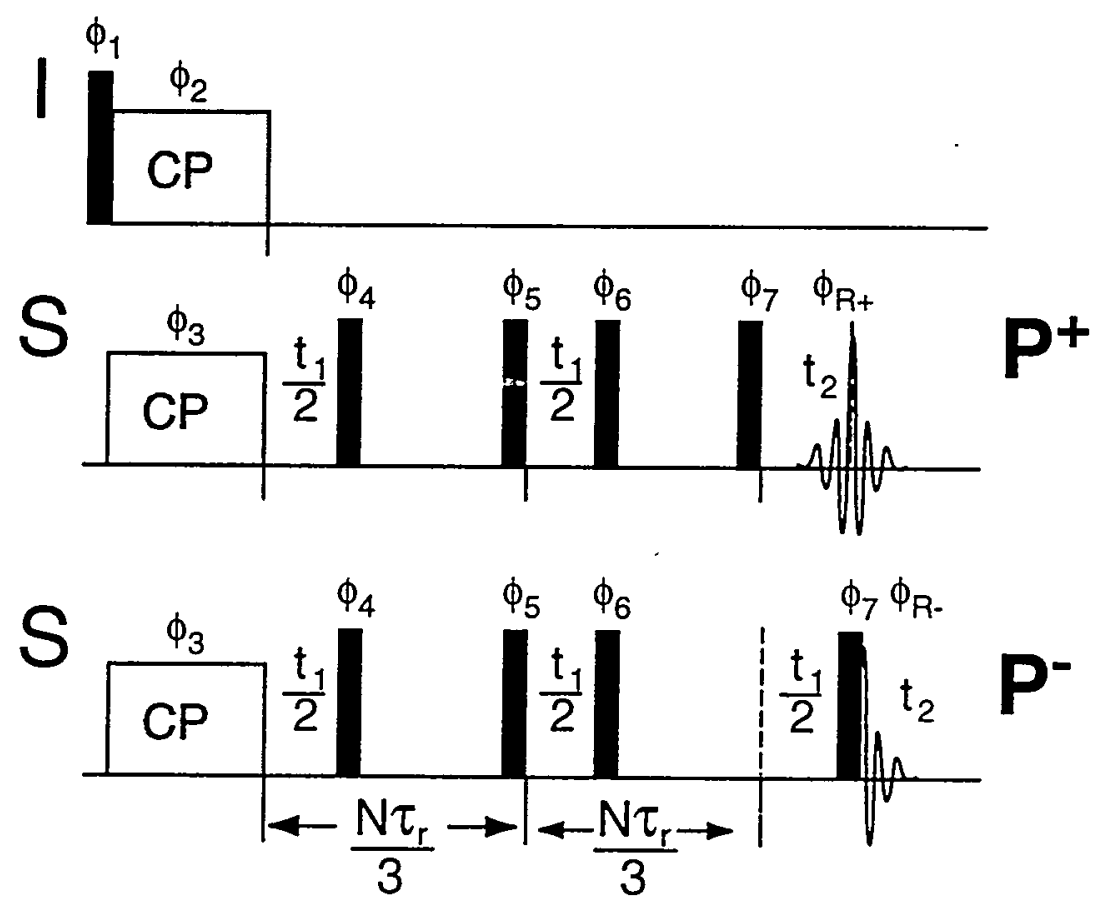

$\begin{array}{lllllllll}\phi_{1}: & 0202 & 0202 & 0202 & 0202 & 0202 & 0202 & 0202 & 0202 \\ \phi_{2}: & 1111 & 1111 & 1111 & 1111 & 1111 & 1111 & 1111 & 1111 \\ \phi_{3}: & 0000 & 0000 & 0000 & 0000 & 0000 & 0000 & 0000 & 0000 \\ \phi_{4}: & 3300 & 3300 & 3300 & 3300 & 3300 & 3300 & 3300 & 3300 \\ \phi_{5}: & 1111 & 1111 & 1111 & 1111 & 1111 & 1111 & 1111 & 1111 \\ \phi_{6}: & 3300 & 0033 & 3300 & 0033 & 3300 & 0033 & 3300 & 0033 \\ \phi_{7}: & 1111 & 1111 & 2222 & 2222 & 3333 & 3333 & 0000 & 0000 \\ \phi_{R_{+}}: & 0220 & 3131 & 1331 & 0202 & 2002 & 1313 & 3113 & 2020 \\ \phi_{R_{-}}: & 0220 & 1313 & 1331 & 2020 & 2002 & 3131 & 3113 & 0202\end{array}$

Figure 5.4 - Pure-phase pulse sequence for isotropic-anisotropic correlation spectroscopy using only $90^{\circ}$ pulses. ${ }^{183}$ Spin-temperature alternation ${ }^{47}$ and CYCLOPS ${ }^{46}$ have been added to the originally published phase cycle. When applied to organic samples, heteronuclear decoupling must be used during the evolution and detection periods. Details are described in the text. 
By using $180^{\circ}$ pulses, it is possible to avoid the $\mathrm{z}$-filters (and the loss of magnetization that goes along with them) and keep all of the magnetization in the transverse plane. Methods for doing this will be described in the following section.

\subsubsection{MAT with $180^{\circ}$ Pulses}

Variations of the MAT experiment which use $180^{\circ}$ pulses rather than $90^{\circ}$ pulses provide a convenient alternative for samples with sufficiently long $T_{2}$ times. ${ }^{185}$ As shown in Section 5.3.1, such experiments take advantage of the same symmetry properties of second-rank tensors that the MAH experiment exploits. By keeping the magnetization in the xy-plane, the loss of magnetization that comes from using a $z$-filter is avoided.

Figure 5.5 shows one such pulse sequence developed by Gann (not to be confused with Gan) et al,. ${ }^{179}$ and Figure 5.6 shows a spectrum of low albite acquired with this sequence. While the sensitivity of the spectrum is quite good, the resolution is rather poor. This is due to the fact that the experiment is a constant-time experiment, which limits the maximum value of $t_{1}$, leading to truncation artifacts. From the pulse spacing in Figure 5.5 , it is easy to see that $t_{1}$ must be less than $2 N \tau_{\Gamma}$ Increasing the value of $N$ leads to improved resolution for a given dwell time, and the value of $\mathrm{N}$ also must be large enough that the entire echo can be acquired if one wishes to avoid phase distortions. However, due to $\mathrm{T}_{2}$ relaxation, there is a practical limit on the total number of rotor cycles which can be used before the sensitivity becomes prohibitively low.

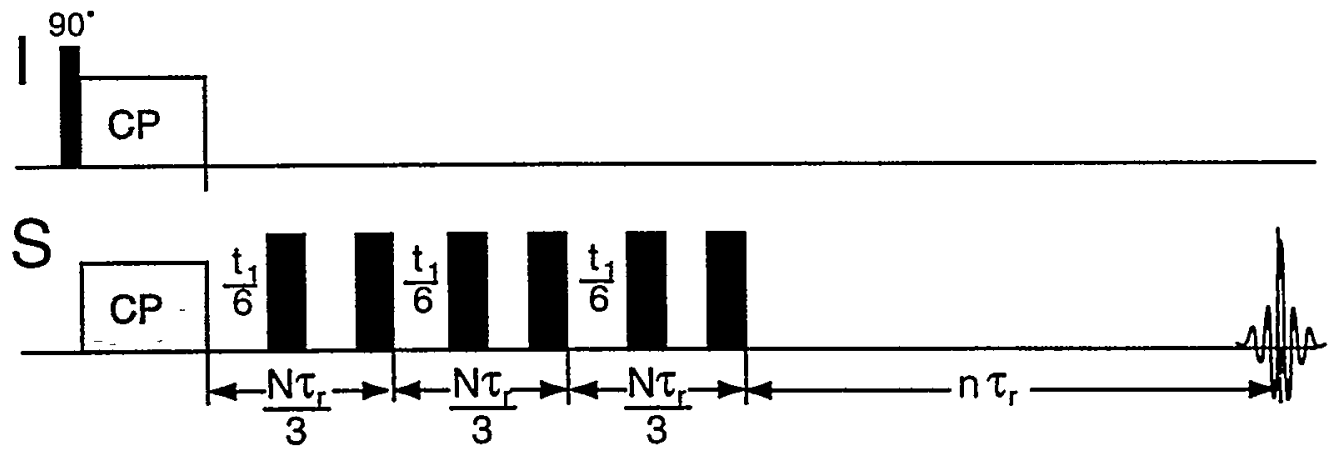

Figure 5.5 - Pulse sequence of Gann et al. ${ }^{179}$ for performing an MAT experiment with $180^{\circ}$ pulses. The $180^{\circ}$ pulses are phase cycled in steps of $180^{\circ}$ to retain the \pm 1 coherences at all times. Spin-temperature alternation ${ }^{47}$ and CYCLOPS ${ }^{46}$ are also used. An echo is formed for $n=N$ as described in Section 5.3.1. Because the whole echo is acquired for all $t_{1}$ points, only a single data set needs to be collected. 
The spectrum shown in Figure 5.6 was acquired with $\mathrm{N}=1$. Interestingly, the most intense peak in the $t_{2}$ dimension occurred at two rotor periods after the last $180^{\circ}$ pulse rather than one. The reason for this artifact is not yet understood, though it may be related to the fact that the number of rotor cycles over which the experiment was performed was quite small, causing one of the rotational echoes to be coincident with the last $180^{\circ}$ pulse. Pulse-length or timing imperfections and other sources of experimental error also cannot be ruled out. The data was processed using whole-echo acquisition (see Section 1.5.2) with the most intense peak used as the center of the echo. Surprisingly, using the "wrong" echo gave results which were in good agreement with those obtained from other pulse sequences. This was most likely due to the fact that the apodization applied to the $t_{2}$ dimension suppressed much of the signal from the other rotational echoes, so the majority of the signal was determined by one rotary echo. Since all of the rotary echoes have the same shape (differing from each other only by a phase shift), ${ }^{39}$ similar isotropicanisotropic correlation spectra can, in principle, be obtained from any one of them. ${ }^{183}$ Still, the results obtained from this method should be viewed as suspect until the nature of the artifact is fully understood.

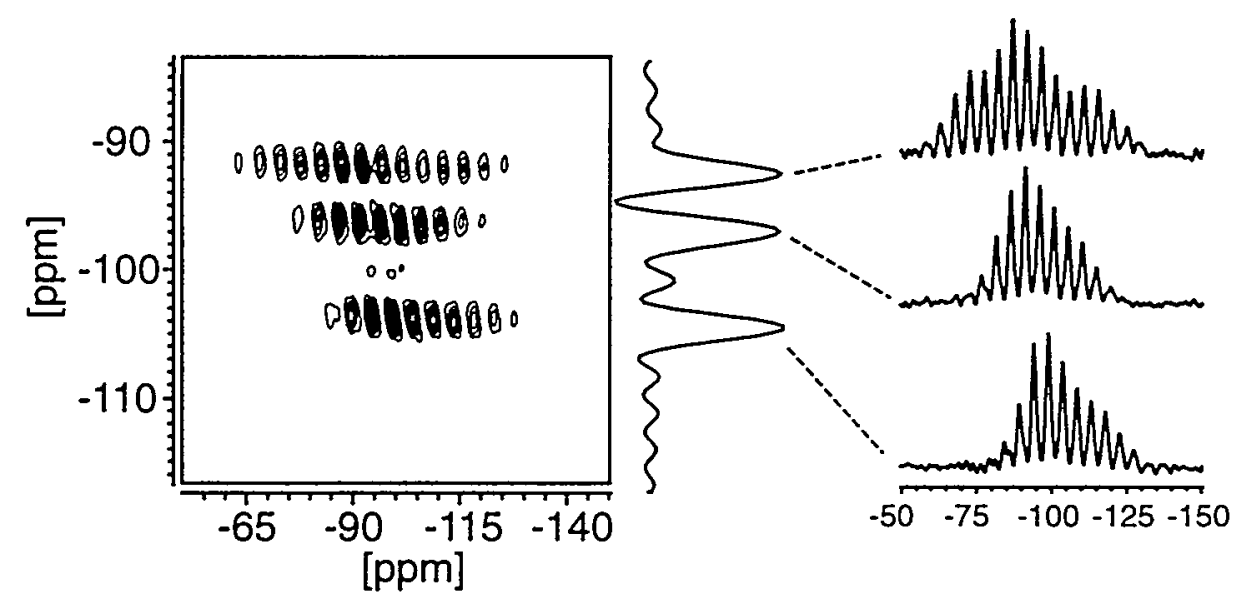

Figure 5.6 - Isotropic/anisotropic correlation spectrum of low albite acquired using the pulse sequence of Figure 5.5. The dwell time in the $t_{1}$ dimension was $300 \mu$ s while that in the $t_{2}$ dimension was $100 \mu \mathrm{s}$. Fifteen $t_{1}$ points (with 1024 scans in each) and $512 t_{2}$ points were acquired, but the data was zero filled to form a $128 \times 512$ data set. The total experimental time was 21.5 hours. The spinning speed was $470 \mathrm{~Hz}$ and ${ }^{29} \mathrm{Si} 180^{\circ}$ pulse length was $28 \mu \mathrm{s}$. The experiment was performed with $\mathrm{N}=1$. 


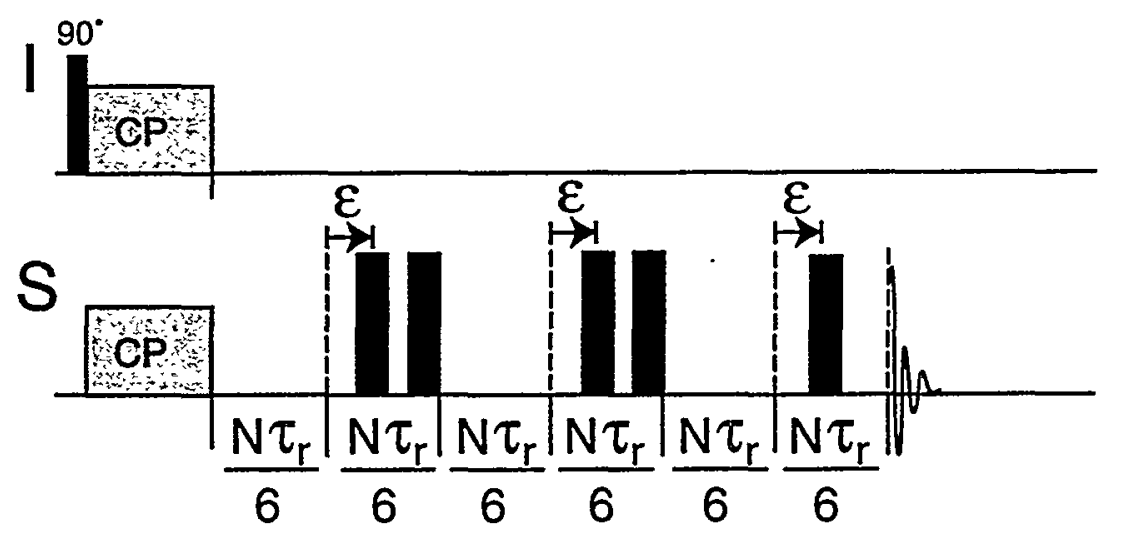

Figure 5.7 - Pulse sequence of Hu et al..$^{180}$ for performing the MAT experiment with $180^{\circ}$ pulses. Two data sets are collected corresponding to positive and negative increments of $\varepsilon$, and the data are processed as described in the text. Phases are cycled as described in the caption of Figure 5.5.

Another method for performing the MAT experiment with $180^{\circ}$ pulses was developed by Hu et al. and is shown in Figure 5.7. ${ }^{180}$ As in Gann's experiment, a series of $180^{\circ}$ pulses is used to keep the magnetization in the xy-plane at all times. To obtain purephase spectra with this sequence, two data sets must be recorded. These data sets are acquired with the same phase cycle and differ only in the spacing between pulses. In one data set, the time between the cross polarization pulse and the first $180^{\circ}$ pulse is given by $\left(N \tau_{r} / 6+\varepsilon\right)$ while in the other data set it is given by $\left(N \tau_{r} / 6-\varepsilon\right)$. In both cases, the value of $\varepsilon$ is incremented from slice to slice by one-sixth of the $t_{1}$-dimension dwell time. A linear combination of the data sets gives the amplitude-modulated sine and cosine data sets, which can then be processed in the usual way.

An experiment that uses hypercomplex data acquisition will be less sensitive (by a factor of $\sqrt{2}$ ) than one that uses whole-echo acquisition since twice as many data points must be acquired to obtain pure-phase spectra. However, it is not necessary to wait several rotor periods for the formation of an echo. The experiment of Hu et al. is also a constant time experiment ${ }^{180}$ and is subject to a more stringent condition $\left(t_{1}<N \tau_{r}\right)$ than that of Gann et al. ${ }^{179}$ Figure 5.8 shows a spectrum acquired with $\mathrm{N}=4$. 


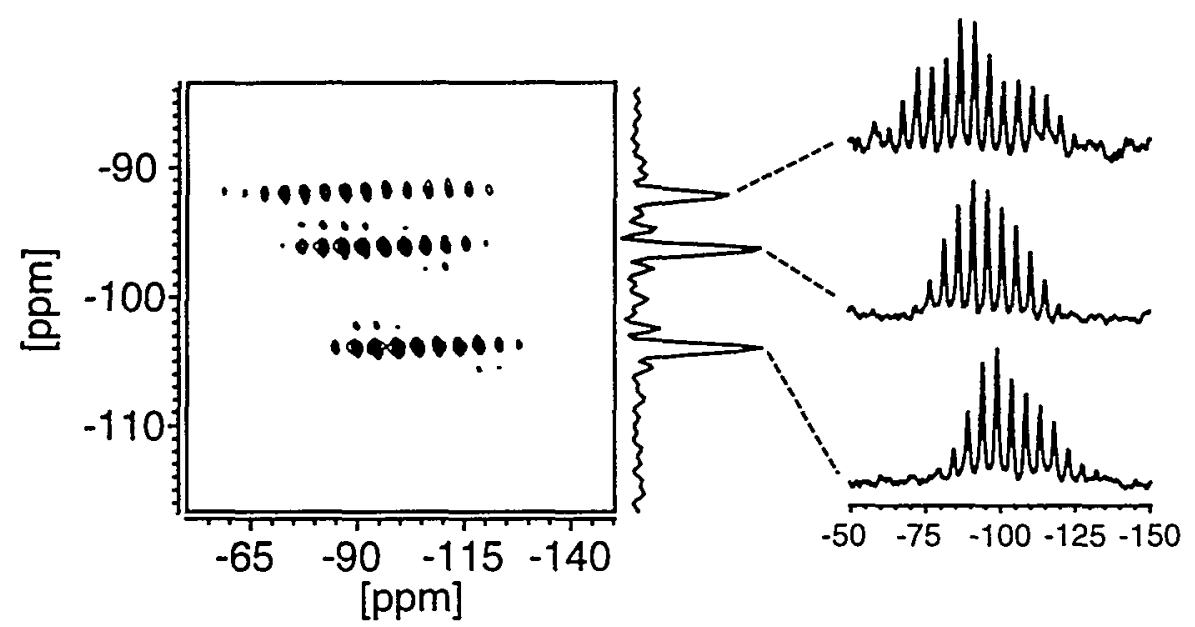

Figure 5.8 - Isotropic/anisotropic correlation spectrum of low albite acquired using the pulse sequence of Figure 5.7. The dwell time in the $t_{1}$ dimension was $300 \mu$ s while that in the $t_{2}$ dimension was $100 \mu \mathrm{s}$. Fifty-seven hypercomplex $t_{1}$ points (with 512 scans in each) and $512 \mathrm{t}_{2}$ points were acquired, but the data was zero filled to form a $128 \times 512$ data set. The total experimental time was 82 hours. The spinning speed was $470 \mathrm{~Hz}$ and ${ }^{29} \mathrm{Si} 180^{\circ}$ pulse length was $28 \mu \mathrm{s}$. The experiment was performed with $\mathrm{N}=4$.

\subsubsection{TOSS-reTOSS}

MAT is not the only way to obtain isotropic-anisotropic correlation spectra of spinning samples. Another method ${ }^{174}$ makes use of a technique known as TOtal Suppression of Sidebands (TOSS). ${ }^{182,187}$ Like the versions of MAT which use $180^{\circ}$ pulses, TOSS exploits the fact that $180^{\circ}$ pulses can generate echoes of spin components while sample spinning generates rotational echoes.

By cleverly positioning the $180^{\circ}$ pulses, one can allow the spin echoes and their corresponding rotational echo manifolds to interfere in such a way that the anisotropic contributions to the FID cancel out, and the isotropic contributions (the centerbands of the frequency spectrum) add constructively over the powder. ${ }^{182}$ Unlike in the case of MAT, this constructive interference is not a true echo, and the magnetization vectors themselves are not aligned at that point. Consequently, rotational echoes will not be formed. ${ }^{169.188}$ The conditions for sideband suppression can be determined by looking at the total phase evolution $^{23}$ as was done in the MAT case 


$$
\begin{aligned}
\Phi\left(\tau^{\prime}\right)= & \int_{0}^{a} \Omega(t) d t-\int_{a}^{a+b} \Omega(t) d t+\int_{a+b}^{a+b+c} \Omega(t) d t \\
& -\int_{a+b+c}^{a+b+c+d} \Omega(t) d t+\int_{a+b+c+d}^{a+b+c+d+c} \Omega(t) d t
\end{aligned}
$$

where the $180^{\circ}$ pulses change the sign of the phase. The goal is to find a set of pulse spacings $\{a, b, c, d, e\}$ that causes the anisotropies to cancel out for all crystallites. In addition, the spacings are chosen so that at the end of the fifth interval, the isotropic echo is also refocused. Beginning acquisition at that point permits an FID that corresponds to a sideband-free spectrum to be recorded. Many possible sets of pulse spacings have been given in the literature; ${ }^{182,189}$ one set of values is listed in the caption of Figure 5.9.

The trajectories of the crystallites can be refocused by applying the TOSS sequence in reverse. Kolbert et al. ${ }^{169}$ showed that by inserting an $t_{1}$ evolution period between a TOSS sequence and a reversed TOSS sequence, an isotropic-anisotropic correlation spectrum can be recorded. This sequence is known as TOSS-reversedTOSS (TOSS-reTOSS or TOSS-“deTOSS”) and is shown in Figure 5.9a.

Like the versions of MAT that use $180^{\circ}$ pulses, the TOSS-reTOSS experiment can only be used on samples with $\mathrm{T}_{2}$ 's that are longer than several rotor periods, and all three experiments are susceptible to artifacts from imperfect $180^{\circ}$ pulses. The TOSS-reTOSS is not a constant time experiment which means there is no restriction on $t_{1}$. This does not prove to be much of an advantage compared to the pulse sequence of Figure 5.7 since the TOSS and reTOSS steps themselves have a combined duration of $4.5 \tau_{\mathrm{r}}$ before the first $t_{1}$ point is even taken. A disadvantage of the TOSS-reTOSS experiment is the fact that it produces phase-twist lineshapes ${ }^{191}$ (see Figure 5.10 ). This can be remedied by performing an experiment with whole-echo acquisition as shown in Figure 5.11. Another potential problem with TOSS-reTOSS is the question of the centerband intensity which is distorted in the TOSS experiment. ${ }^{169}$ The reverse TOSS part of the sequence is predicted to undo this distortion, however. ${ }^{191}$ In fitting the TOSS-reTOSS experiments performed with a 

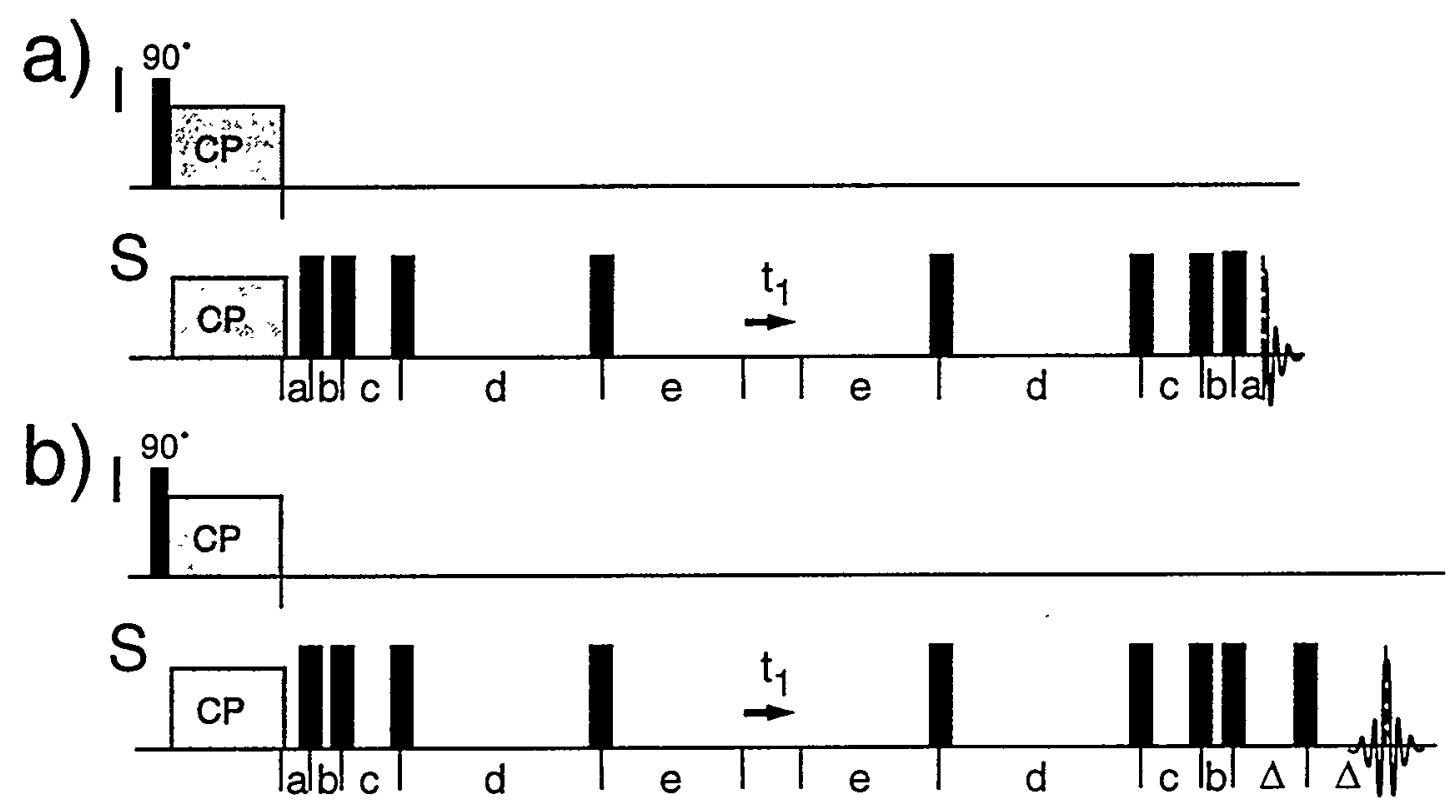

Figure 5.9 - (a) Original TOSS-reTOSS sequence ${ }^{169}$ for isotropic-anisotropic correlation spectroscopy. No phase cycling was applied to the $180^{\circ}$ pulses since phase-cycling does not affect the efficiency of TOSS. ${ }^{190}$ (b) TOSS-reTOSS combined with whole-echo acquisition for obtaining pure-phase spectra. The $180^{\circ}$ pulses are cycied in steps of four to retain only the $p=-1$ coherence. The timings in both sequences are given by: $a=0.1226 \tau_{\Gamma}$, $b=0.0773 \tau_{\Gamma}, c=0.2236 \tau_{\Gamma} d=1.0433 \tau_{r}$ and $e=0.7744 \tau_{r}$ The echo delay must satisfy the condition $\Delta=\mathrm{a}+\mathrm{n} \tau_{\mathrm{r}}$ where $\mathrm{n}$ is an integer.

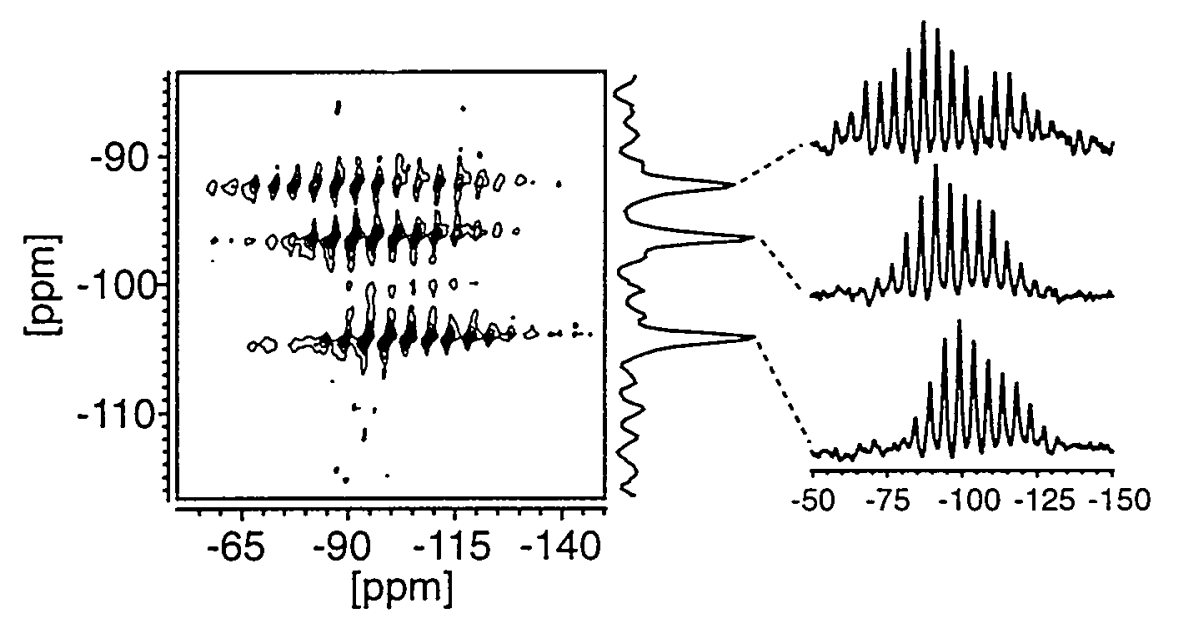

Figure 5.10 - Isotropic/anisotropic correlation spectrum of low albite acquired using the pulse sequence of Figure $5.9 \mathrm{a}$. The dwell time in the $\mathrm{t}_{1}$ dimension was $300 \mu \mathrm{s}$ while that in the $t_{2}$ dimension was $100 \mu \mathrm{s}$. Sixty-one hypercomplex $t_{1}$ points (with 512 scans in each) and $512 t_{2}$ points were acquired, but the data was zero filled to form a $128 \times 512$ data set. The total experimental time was 88 hours. The spinning speed was $470 \mathrm{~Hz}$ and ${ }^{29} \mathrm{Si}$ $180^{\circ}$ pulse length was $34 \mu \mathrm{s}$. 
spinning speed of $680 \mathrm{~Hz}$ (see Tables 5.1, 5.2, and 5.3), the centerband intensity was excluded from the fits. It was included in the fits of the data acquired with a spinning speed of $470 \mathrm{~Hz}$. No systematic effect was observed.

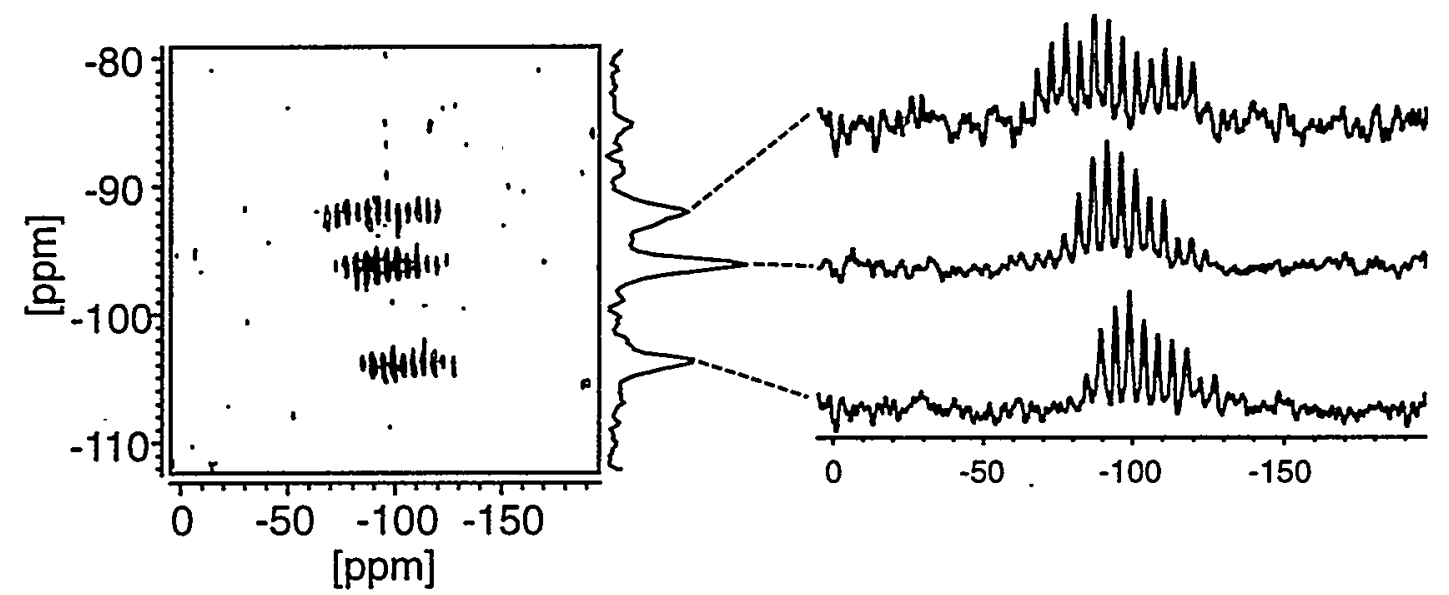

Figure 5.11 - Isotropic/anisotropic correlation spectrum of low albite acquired using the pulse sequence of Figure 5.9b. The dwell time in the $t_{1}$ dimension was $300 \mu \mathrm{s}$ while that in the $t_{2}$ dimension was $100 \mu \mathrm{s}$. Sixty-four $t_{1}$ points (with 512 scans) and $1024 t_{2}$ points were acquired, but the data was zero filled to form a $128 \times 512$ data set. The total experimental time was 46 hours. The spinning speed was $470 \mathrm{~Hz}$ and ${ }^{29} \mathrm{Si} 180^{\circ}$ pulse length was $34 \mu \mathrm{s}$. The time allowed for echo formation was $5 \tau_{\mathrm{r}}=10.6 \mathrm{~ms}$.

\subsection{Application of Isotropic-Anisotropic Correlation Methods to Low Albite}

The isotropic-anisotropic correlation spectra presented in Figures 5.6, 5.8, 5.10, and 5.11 were recorded under similar experimental conditions. All used a spinning speed of $470 \mathrm{~Hz}$ (controlled with a home-built spinning speed controller), a dwell time in the $t_{1}$ dimension of $300 \mu \mathrm{s},{ }^{27} \mathrm{Al}$ excitation field strengths of $4.5-6 \mathrm{kHz},{ }^{29} \mathrm{Si} 180^{\circ}$ pulse field strengths of $2.4-2.8 \mathrm{kHz}$, and a recycle delay of $5 \mathrm{~s}$ between scans. Other parameters are listed in the figure captions. The signal-to-noise was calibrated by recording onedimensional spectra before and after each two-dimensional experiment; to within experimental error, the intrinsic signal-to-noise ratio was constant in all experiments. Variations in the quality of the two-dimensional spectra are due to different methods of data acquisition, different numbers of $t_{1}$ points recorded, and pulse-sequence-dependent artifacts as discussed in Section 5.3. All data were apodized with exponential line broadenings of $100 \mathrm{~Hz}$ in the anisotropic dimension and $50 \mathrm{~Hz}$ in the isotropic dimension. 
The $t_{1}$ dimension was zero-filled to 128 points in all cases. The number of $t_{2}$ points was 512 for all experiments except the TOSS-reTOSS experiment with whole echo acquisition where 1024 points were used. The contours levels in all four figures range from 10 to $100 \%$ in steps of $10 \%$. The spectra were referenced to an external standard of TMS.

The cross-polarization conditions were optimized experimentally. At $\omega_{\mathrm{r}} /(2 \pi)=470 \mathrm{~Hz}$, the best ${ }^{27} \mathrm{Al}$ spin lock (in the sudden regime) occurred at a field strength of $1070 \mathrm{~Hz}$. The ${ }^{29} \mathrm{Si}$ spin lock field strength was matched to it empirically, and optimum contact times were found to be $8 \mathrm{~ms}$.

The principal values of the CSA were extracted by performing Herzfeld-Berger ${ }^{40}$ fits. Spectra acquired with a spinning speed of $680 \mathrm{~Hz}$ (data not shown) were fit using the program Speedyfit ${ }^{99}$ provided by Dr. H. J. M. de Groot. The program used HerzfeldBerger lookup tables to simulate spinning sideband manifolds and can fit spectra with overlapping sites provided they have eight or fewer sidebands. Figure 5.12 shows an
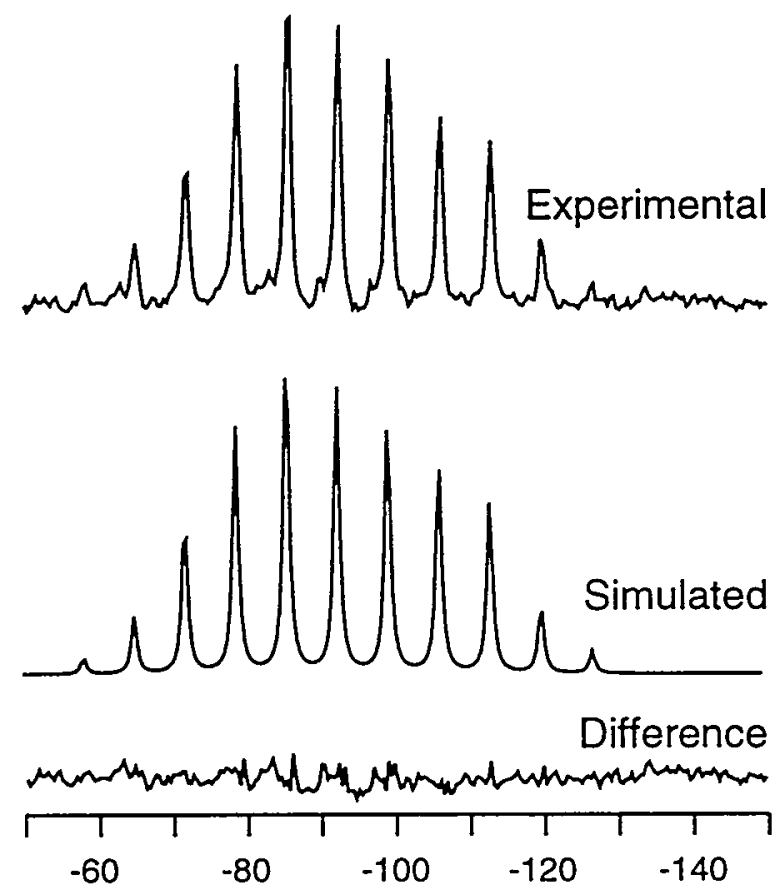

Figure 5.12 - Typical results of the Speedyfit ${ }^{99}$ program for determining principal values of the CSA tensor from MAS spectra. The experimental spectrum corresponds to the $91.8 \mathrm{ppm}$ site as recorded using the pulse sequence of Figure 5.7 with a spinning speed of $680 \mathrm{~Hz}$. The results are listed in the fourth row of Table 5.1 . 
example of a typical fit from this program. Spectra acquired with a spinning speed of 470 $\mathrm{Hz}$ had more sidebands and were fit using a program that utilized Floquet theory and was written with GAMMA ${ }^{130}$ by Dr. Matthias Ernst.

\begin{tabular}{|c|c|c|c|c|c|c|}
\hline $\begin{array}{c}\text { pulse } \\
\text { sequence }\end{array}$ & $\omega_{\mathbf{r}} /(\mathbf{2 \pi})$ & $\begin{array}{c}\sigma_{\mathbf{1 1}} \\
{[\mathbf{p p m}]}\end{array}$ & $\begin{array}{c}\sigma_{\mathbf{2 2}} \\
{[\mathbf{p p m}]}\end{array}$ & $\begin{array}{c}\sigma_{33} \\
{[\mathbf{p p m}]}\end{array}$ & $\eta^{\mathbf{C S}}$ & $\begin{array}{c}\delta^{\mathbf{C S}} \\
{[\mathbf{p p m}]}\end{array}$ \\
\hline \hline Figure 5.5 & $470 \mathrm{~Hz}$ & $-61 \pm 3$ & $-88 \pm 3$ & $-126 \pm 3$ & $0.8 \pm 0.1$ & $-35 \pm 4$ \\
\hline Figure 5.5 & $680 \mathrm{~Hz}$ & $-60 \pm 7$ & $-88 \pm 6$ & $-127 \pm 8$ & $0.8 \pm 0.3$ & $-36 \pm 8$ \\
\hline Figure 5.7 & $470 \mathrm{~Hz}$ & $-62 \pm 2$ & $-89 \pm 1$ & $-125 \pm 3$ & $0.8 \pm 0.1$ & $-33 \pm 3$ \\
\hline Figure 5.7 & $680 \mathrm{~Hz}$ & $-64 \pm 4$ & $-90 \pm 3$ & $-122 \pm 4$ & $0.9 \pm 0.2$ & $-30 \pm 5$ \\
\hline Figure 5.9a & $470 \mathrm{~Hz}$ & $-54 \pm 7$ & $-89 \pm 3$ & $-132 \pm 9$ & $0.9 \pm 0.3$ & $-40 \pm 9$ \\
\hline Figure 5.9a & $680 \mathrm{~Hz}$ & $-60 \pm 4$ & $-88 \pm 3$ & $-127 \pm 4$ & $0.8 \pm 0.2$ & $-35 \pm 4$ \\
\hline Figure 5.9b & $470 \mathrm{~Hz}$ & $-62 \pm 1$ & $-86 \pm 6$ & $-127 \pm 7$ & $0.7 \pm 0.2$ & $-35 \pm 7$ \\
\hline \hline average & N/A & $-60 \pm 3$ & $-88 \pm 1$ & $-127 \pm 3$ & $0.8 \pm 0.1$ & $-35 \pm 3$ \\
\hline
\end{tabular}

Table 5.1 - Principal values of the ${ }^{29} \mathrm{Si} \mathrm{T} 2 \mathrm{~m}$ site $(-91.8 \mathrm{ppm})$ in low albite as determined by various isotropic-anisotropic correlation techniques using ${ }^{27} \mathrm{Al}-$ to- $^{29} \mathrm{Si} \mathrm{CP}$. The error bars represent the accuracy of the fit and not the inherent accuracy of the pulse sequence. The unweighted average and standard deviation of all experiments is given in the last row.

\begin{tabular}{|c|c|c|c|c|c|c|}
\hline $\begin{array}{c}\text { pulse } \\
\text { sequence }\end{array}$ & $\omega_{\mathbf{r}} /(2 \pi)$ & $\begin{array}{c}\sigma_{11} \\
{[\mathbf{p p m}]}\end{array}$ & $\begin{array}{c}\sigma_{22} \\
{[\mathbf{p p m}]}\end{array}$ & $\begin{array}{c}\sigma_{33} \\
{[\mathbf{p p m}]}\end{array}$ & $\eta^{\mathbf{C S}}$ & $\begin{array}{c}\delta^{\mathrm{CS}} \\
{[\mathbf{p p m}]}\end{array}$ \\
\hline \hline Figure 5.5 & $470 \mathrm{~Hz}$ & $-77 \pm 3$ & $-94 \pm 3$ & $-118 \pm 3$ & $0.8 \pm 0.2$ & $-22 \pm 4$ \\
\hline Figure 5.5 & $680 \mathrm{~Hz}$ & $-73 \pm 7$ & $-93 \pm 5$ & $-123 \pm 7$ & $0.7 \pm 0.3$ & $-27 \pm 7$ \\
\hline Figure 5.7 & $470 \mathrm{~Hz}$ & $-76 \pm 2$ & $-95 \pm 1$ & $-118 \pm 2$ & $0.9 \pm 0.2$ & $-21 \pm 3$ \\
\hline Figure 5.7 & $680 \mathrm{~Hz}$ & $-74 \pm 2$ & $-93 \pm 1$ & $-121 \pm 2$ & $0.8 \pm 0.1$ & $-25 \pm 2$ \\
\hline Figure 5.9a & $470 \mathrm{~Hz}$ & $-75 \pm 3$ & $-94 \pm 3$ & $-119 \pm 3$ & $0.9 \pm 0.2$ & $-23 \pm 4$ \\
\hline Figure 5.9a & $680 \mathrm{~Hz}$ & $-72 \pm 4$ & $-93 \pm 3$ & $-123 \pm 4$ & $0.8 \pm 0.2$ & $-27 \pm 4$ \\
\hline Figure 5.9b & $470 \mathrm{~Hz}$ & $-76 \pm 1$ & $-94 \pm 1$ & $-118 \pm 1$ & $0.8 \pm 0.1$ & $-22 \pm 1$ \\
\hline \hline average & N/A & $-75 \pm 2$ & $-94 \pm 1$ & $-120 \pm 2$ & $0.8 \pm 0.1$ & $-22 \pm 2$ \\
\hline
\end{tabular}

Table 5.2 - Principal values of the ${ }^{29} \mathrm{Si}$ T2O site $(-96.1 \mathrm{ppm})$ in low albite as determined by various isotropic-anisotropic correlation techniques using ${ }^{27} \mathrm{Al}-\mathrm{to}^{29} \mathrm{Si} \mathrm{CP}$. The unweighted average and standard deviation of all experiments is given in the last row 


\begin{tabular}{|c|c|c|c|c|c|c|}
\hline $\begin{array}{c}\text { pulse } \\
\text { sequence }\end{array}$ & $\omega_{\boldsymbol{}} /(2 \pi)$ & $\begin{array}{c}\sigma_{\mathbf{1 1}} \\
{[\mathbf{p p m}]}\end{array}$ & $\begin{array}{c}\sigma_{\mathbf{2 2}} \\
{[\mathbf{p p m}]}\end{array}$ & $\begin{array}{c}\sigma_{33} \\
{[\mathbf{p p m}]}\end{array}$ & $\eta^{\mathrm{CS}}$ & $\begin{array}{c}\delta^{\mathrm{CS}} \\
{[\mathbf{p p m}]}\end{array}$ \\
\hline \hline Figure 5.5 & $470 \mathrm{~Hz}$ & $-85 \pm 3$ & $-100 \pm 3$ & $-127 \pm 3$ & $0.6 \pm 0.2$ & $-23 \pm 4$ \\
\hline Figure 5.5 & $680 \mathrm{~Hz}$ & $-80 \pm 5$ & $-98 \pm 5$ & $-134 \pm 6$ & $0.6 \pm 0.3$ & $-30 \pm 6$ \\
\hline Figure 5.7 & $470 \mathrm{~Hz}$ & $-85 \pm 2$ & $-100 \pm 1$ & $-127 \pm 2$ & $0.7 \pm 0.1$ & $-23 \pm 3$ \\
\hline Figure 5.7 & $680 \mathrm{~Hz}$ & $-82 \pm 2$ & $-99 \pm 2$ & $-131 \pm 2$ & $0.6 \pm 0.1$ & $-27 \pm 2$ \\
\hline Figure 5.9a & $470 \mathrm{~Hz}$ & $-83 \pm 3$ & $-101 \pm 3$ & $-128 \pm 4$ & $0.8 \pm 0.2$ & $-24 \pm 5$ \\
\hline Figure 5.9a & $680 \mathrm{~Hz}$ & $-81 \pm 3$ & $-99 \pm 3$ & $-133 \pm 3$ & $0.6 \pm 0.2$ & $-29 \pm 3$ \\
\hline Figure 5.9b & $470 \mathrm{~Hz}$ & $-83 \pm 1$ & $-100 \pm 1$ & $-128 \pm 1$ & $0.7 \pm 0.1$ & $-24 \pm 1$ \\
\hline \hline average & N/A & $-83 \pm 2$ & $-100 \pm 1$ & $-130 \pm 3$ & $0.7 \pm 0.1$ & $-26 \pm 3$ \\
\hline
\end{tabular}

Table 5.3 - Principal values of the ${ }^{29} \mathrm{Si} \mathrm{T} 1 \mathrm{~m}$ site $(-103.9 \mathrm{ppm})$ in low albite as determined by various isotropic-anisotropic correlation techniques using ${ }^{27} \mathrm{Al}-{ }^{20}-{ }^{29} \mathrm{Si} \mathrm{CP}$. The unweighted average and standard deviation of all experiments is given in the last row.

The results of fits from all four experiments are listed in Tables 5.1, 5.2, and 5.3. Summations over several $\omega_{1}$ slices were used to produce anisotropic spectra for fitting except in the case of the poorly phased TOSS-reTOSS spectra where only one slice was used. To within experimental error, all four pulse sequences give similar results for low albite. The error bars reflect the quality of the fits and not the intrinsic accuracy of the experiment. Due to the small number of experiments performed and the absence of an independent and accurate way to directly measure the ${ }^{29} \mathrm{Si}$ CSA parameters in low albite, no general conclusions about the reliability of the pulse sequences could be drawn.

Correlations between anisotropic chemical-shift parameters and number of bridging oxygens have been reported for $\mathrm{Q}^{\mathrm{n}}(\mathrm{OAl})$ sites, ${ }^{192,193}$ but the influence of ${ }^{27} \mathrm{Al}$ on the ${ }^{29} \mathrm{Si}$ anisotropy parameters is not currently understood. To date, few such compounds have been measured, and $a b$ initio calculations on silicates are in their infancy. ${ }^{194}$ Cross polarization from quadrupolar nuclei to ${ }^{29} \mathrm{Si}$ may enable CSA parameters to be determined for a variety of silicates, providing a database for empirical correlations. 


\subsection{Experiments on Low Microcline}

Principal values of the chemical-shielding anisotropy have also been determined for the mineral low microcline $\left(\mathrm{KAlSi}_{3} \mathrm{O}_{8}\right)$ by performing isotropic-anisotropic correlation experiments which utilized ${ }^{27} \mathrm{Al}-{ }^{-t o}-{ }^{29} \mathrm{Si}$ cross polarization. Low microcline has the same framework structure as low albite (see Figure 3.1) and analogous peak assignments. ${ }^{117}$ Cross-polarization experimental parameters (e.g. - optimum power levels and contact times) for the isotropic-anisotropic correlation experiments were similar to those of low albite (see Section 5.4), and as in the case of albite, the cross-polarized signal for the $\mathrm{Q}^{4}(2 \mathrm{Al})$ site (at $-94.0 \mathrm{ppm}$ ) was consistently less intense than that of one of the $\mathrm{Q}^{4}(1 \mathrm{Al})$ sites (at $-99.5 \mathrm{ppm}$ ) for a range of spinning speeds. (The other $\mathrm{Q}^{4}(1 \mathrm{Al})$ site could not be used for comparison since the peak contained contributions from albite present in the sample.)

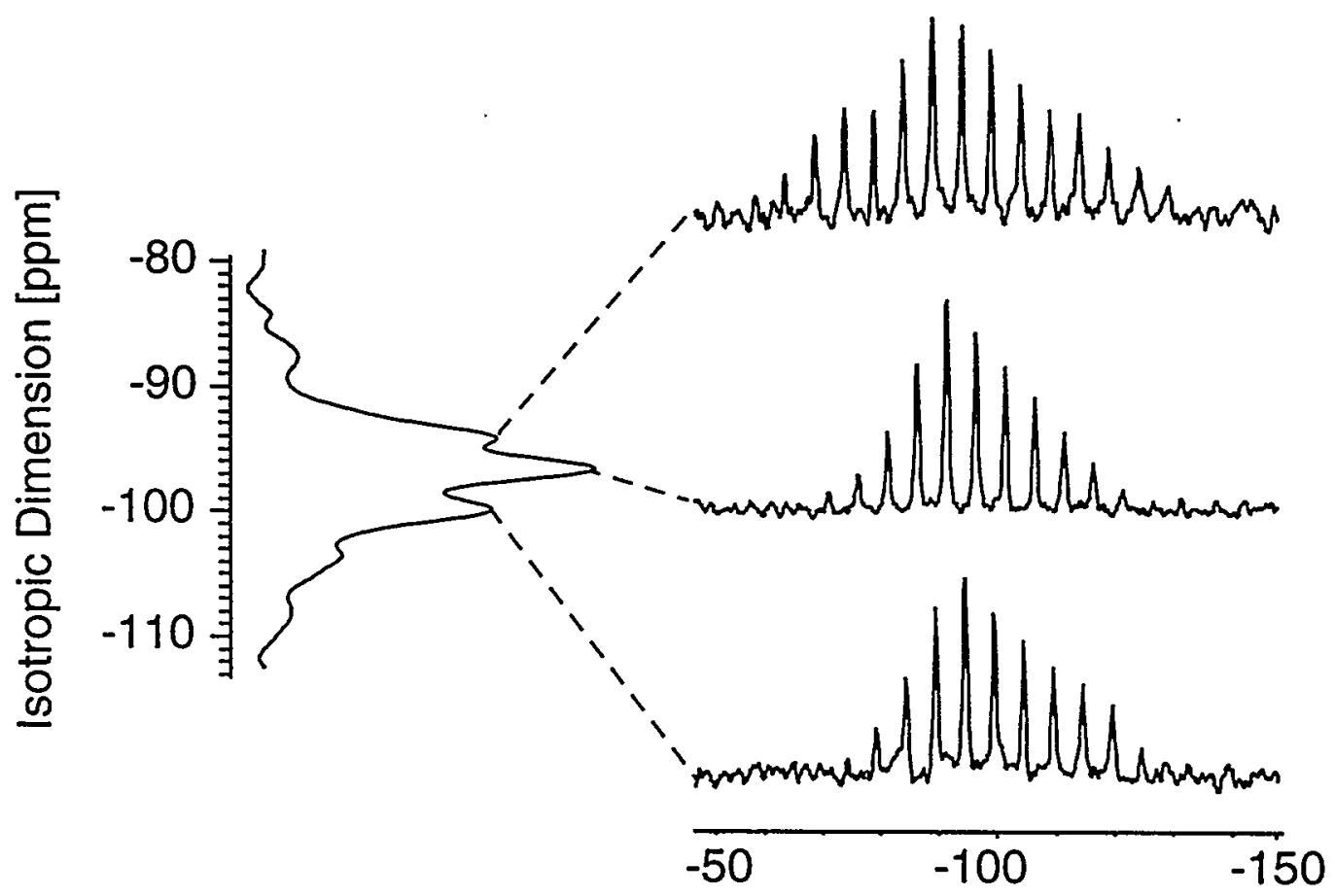

Anisotropic Dimension [ppm fromTMS]

Figure 5.13 - Isotropic and anisotropic spectra of ${ }^{29} \mathrm{Si}$ in low microcline recorded using the pulse sequence of Figure $5.9 \mathrm{~b}$ with ${ }^{27} \mathrm{Al}-\mathrm{to}^{29} \mathrm{Si}$ cross polarization. The spinning speed was $500 \mathrm{~Hz}$, and the ${ }^{29} \mathrm{Si} 180^{\circ}$ pulse length was $58 \mu \mathrm{s}$. Sixty-three $t_{1}$ slices were recorded with 512 scans in each and a recycle delay of $5 \mathrm{~s}$. The delay for formation of the echo was five rotor periods. 
Figure 5.13 shows the isotropic projection and several anisotropic slices from a TOSS-reTOSS experiment (whole-echo version) performed on low microcline. Due to the domains of low albite nearly always found in natural samples of low microcline, ${ }^{195}$ the isotropic ${ }^{29} \mathrm{Si}$ spectrum has many closely spaced peaks. The principal values of the chemical-shielding tensors could not have been determined from a one-dimensional MAS experiment. However, they are easily determined from the two-dimensional spectrum and are summarized in Table 5.4.

\begin{tabular}{|c|c|c|c|c|c|c|}
\hline $\begin{array}{c}\text { Isotropic } \\
\text { Shift } \\
\text { [ppm] }\end{array}$ & $\omega_{\mathbf{r}} /(2 \pi)$ & $\begin{array}{c}\sigma_{11} \\
{[\mathbf{p p m}]}\end{array}$ & $\begin{array}{c}\sigma_{22} \\
{[\mathbf{p p m}]}\end{array}$ & $\sigma_{33}[\mathbf{p p m}]$ & $\eta^{\mathrm{CS}}$ & $\begin{array}{c}\delta^{\mathrm{CS}} \\
{[\mathbf{p p m}]}\end{array}$ \\
\hline \hline-94.0 & $500 \mathrm{~Hz}$ & $-62 \pm 7$ & $-92 \pm 2$ & $-128 \pm 6$ & $0.9 \pm 0.3$ & $-34 \pm 6$ \\
\hline-94.0 & $680 \mathrm{~Hz}$ & $-63 \pm 8$ & $-92 \pm 11$ & $-127 \pm 9$ & $0.9 \pm 0.7$ & $-34 \pm 9$ \\
\hline-96.4 & $500 \mathrm{~Hz}$ & $-77 \pm 3^{\mathrm{a}}$ & $-94 \pm 1^{\mathrm{a}}$ & $-118 \pm 2^{\mathrm{a}}$ & $0.8 \pm 0.1^{\mathrm{a}}$ & $-22 \pm 2^{\mathrm{a}}$ \\
\hline-96.4 & $680 \mathrm{~Hz}$ & $-73 \pm 9^{\mathrm{a}}$ & $-92 \pm 11^{\mathrm{a}}$ & $-125 \pm 10^{\mathrm{a}}$ & $0.7 \pm 0.7^{\mathrm{a}}$ & $-28 \pm 10^{\mathrm{a}}$ \\
\hline-99.5 & $500 \mathrm{~Hz}$ & $-79 \pm 6$ & $-96 \pm 5$ & $-123 \pm 6$ & $0.7 \pm 0.4$ & $-24 \pm 7$ \\
\hline-99.5 & $680 \mathrm{~Hz}$ & $-75 \pm 6$ & $-92 \pm 8$ & $-132 \pm 6$ & $0.5 \pm 0.4$ & $-32 \pm 7$ \\
\hline
\end{tabular}

Table 5.4 - Principle values of ${ }^{29} \mathrm{Si}$ chemical-shielding tensors in low microcline as determined ${ }^{\mathrm{b}}$ from fits of anisotropic slices recorded with the pulse sequence of Figure 5.9b. The corresponding experimental data are presented in Figure 5.13.

a. Since our sample of low microcline, like almost all samples found in nature, ${ }^{195}$ contains domains of nearly pure albite, the peak at $-96.4 \mathrm{ppm}$ is actually due to an overlap of albite and microcline resonances.

b. These values differ from those published by De Paul et al. ${ }^{196}$ due to an error in the original calculation.

\subsection{Validity of Using Cross Polarization from Quadrupolar Nuclei}

One potential concern when using cross polarization from quadrupolar nuclei to determine chemical-shielding anisotropy powder patterns is whether the crosspolarization process significantly distorts such patterns. For spin-1 nuclei such as ${ }^{14} \mathrm{~N}$, the cross-polarization match condition has been shown to be highly dependent on the orientation of the quadrupolar nucleus. ${ }^{197}$ While similar studies have not been performed for cross polarization from odd-half-integer quadrupolar nuclei, the shape of the ${ }^{23} \mathrm{Na}$ central transition powder pattern after a spin lock ${ }^{126}$ has been shown to be distorted in 
some cases due to different effective adiabaticity parameters for individual crystallites. The general trend for $\alpha<1$ is that the low-frequency side of the powder pattern loses intensity. Even when only spin-1/2 nuclei are involved in cross polarization, distorted powder patterns have been observed and attributed to Hartmann-Hahn mismatch and to anisotropic cross-polarization and relaxation rates. ${ }^{163}$

Although we have optimized the spin-lock efficiency so that as many crystallites as possible are available for cross polarization and performed the experiments at the Hartmann-Hahn match, distortions could still have conceivably occurred. To test this, we have recorded slow-spinning, ${ }^{29} \mathrm{Si}$ direct-excitation spectra of low albite at several different spinning speeds. Figure 5.14 shows one such spectrum along with a simulation based on the parameters from the fourth row of Tables 5.1, 5.2, and 5.3. A HerzfeldBerger spinning sideband analysis was performed on a different spectrum (not shown) recorded at a spinning speed of $1065 \mathrm{~Hz}$. Due to baseline distortions, the quality of this

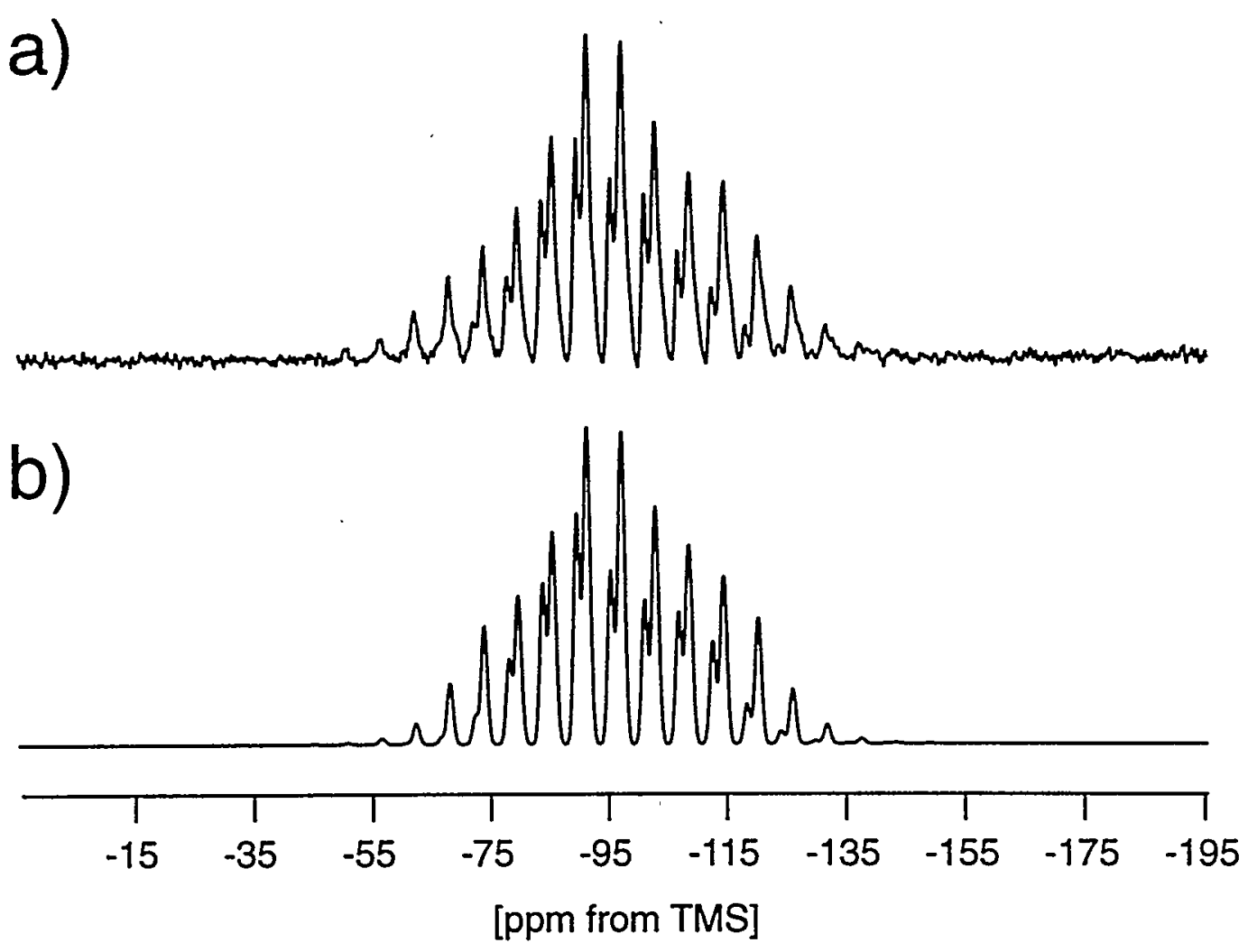

Figure 5.14 - (a) ${ }^{29} \mathrm{Si}$ NMR spectrum of low albite recorded at $11.7 \mathrm{~T}$ with a spinning speed of $575 \mathrm{~Hz}$. Twenty-four scans were acquired with a recycle delay of $2000 \mathrm{~s}$. (b) Simulated ${ }^{29} \mathrm{Si}$ spectrum based on the parameters of the fourth row in Tables 5.1, 5.2, and 5.3. Gaussian lines with widths of $135 \mathrm{~Hz}$ were used to simulate the sidebands. 
spectrum was not high, but the three sites could be partially resolved in several of the sidebands. The results of the analysis are summarized in Table 5.5. At first glance, the

\begin{tabular}{|c|c|c|c|c|c|}
\hline $\begin{array}{c}\text { Isotropic } \\
\text { Shift [ppm] }\end{array}$ & $\sigma_{11}$ [ppm] & $\sigma_{22}$ [ppm] & $\sigma_{\mathbf{3 3}}$ [ppm] & $\eta^{\mathrm{CS}}$ & $\delta^{\mathrm{CS}}[\mathrm{ppm}]$ \\
\hline \hline$-91.9 \pm 0.1$ & $-61 \pm 7$ & $-84 \pm 7$ & $-130 \pm 8$ & $0.6 \pm 0.3$ & $-39 \pm 8$ \\
\hline$-96.1 \pm 0.1$ & $-75 \pm 4$ & $-93 \pm 5$ & $-120 \pm 4$ & $0.8 \pm 0.4$ & $-24 \pm 4$ \\
\hline$-103.8 \pm 0.1$ & $-89 \pm 5$ & $-92 \pm 10$ & $-131 \pm 6$ & $0.1 \pm 0.5$ & $-27 \pm 6$ \\
\hline
\end{tabular}

Table 5.5 - Chemical shielding parameters derived from a Herzfeld-Berger analysis of a ${ }^{29} \mathrm{Si}$ directexcitation MAS spectrum of low albite (spinning speed $=1065 \mathrm{~Hz}$ ).

agreement between these parameters and the previously measured ones (see Tables 5.1, 5.2, and 5.3) does not look very good. However, the error bars for the direct-excitation spectrum are quite substantial due to the severe peak overlap, and except for the value of $\eta^{\mathrm{CS}}$ for the Tlm site, the results from the cross-polarization experiments all fall within the error bars of the values listed in Table 5.5. While this does not rule out the possibility of distortions, it is encouraging that the two data sets are not inconsistent.

\subsection{REDOR Experiments}

Another example of a potential application of cross polarization from quadrupolar nuclei is in heteronuclear experiments between spin-1/2 and quadrupolar nuclei. As mentioned in Section 1.2.4, the strength of the dipolar coupling between two spins is inversely proportional to the cube of the distance between them. Thus, in systems which effectively contain isolated spin pairs, a measurement of the dipolar-coupling constant translates into a direct determination of the internuclear distance. The Spin-Echo DOuble Resonance $(\mathrm{SEDOR})^{5}$ technique is the simplest method for measuring internuclear distances between a pair of unlike nuclei. In the SEDOR experiment, a $90^{\circ}$ pulse generates transverse magnetization on the I spins in a static sample. Application of a $180^{\circ}$ pulse at a time $\tau$ later, will reverse the effects of the heteronuclear dipolar couplings, field inhomogeneities, and the chemical-shift interactions so that they rephase, leading to the formation of an echo at the time $2 \tau$. However, if $180^{\circ}$ pulses are applied to the I and $S$ spins simultaneously, the heteronuclear dipolar coupling continues to dephase during the 
second half of the experiment. Taking the difference between the spectra with and without the $180^{\circ}$ pulse on the $\mathrm{S}$ spins gives a residual signal that is due to the heteronuclear dipolar interaction.

In many cases, the high resolution associated with magic-angle spinning is desirable, thus a technique has been developed by Gullion et al. ${ }^{198,199}$ to measuring heteronuclear distances in rotating samples. This technique is known as Rotational-Echo DOuble Resonance (REDOR). Conceptually, it is similar to SEDOR, but since sample spinning itself averages out the weak heteronuclear interactions, a series of rotorsynchronized $180^{\circ}$ pulses is required to produce the appropriate dephasing and rephasing behavior.

In the most common version of REDOR, ${ }^{200}$ two experiments are performed. In one, a rotor-synchronized Hahn-echo is applied to the spin species which will be detected, and series of $180^{\circ}$ pulses is applied to the other channel every half a rotor period except when the $180^{\circ}$ pulse is applied in the first channel. This sequence will refocus all interactions except for the heteronuclear dipolar interaction. The phase accumulation for a given crystallite can be calculated (in an analogous manner to that of Section 5.3.1 with the relevant interaction now being the heteronuclear dipolar interaction), and from this, the FID of a powder sample can be determined..$^{200}$ In the other experiment, the $180^{\circ}$ pulse on the detected channel is left out, and both dipolar and CSA interactions will be refocused. Taking the difference between the two FIDs and normalizing gives a measure of the heteronuclear dipolar coupling, which can be expressed in an analytical form using Bessel functions ${ }^{201}$

$$
\frac{\Delta S}{S_{0}}=1-\left[\mathrm{J}_{0}\left(\sqrt{2} \mathrm{nd} \tau_{\mathrm{r}}\right)\right]^{2}+2 \sum_{\mathrm{k}=1}^{\infty} \frac{1}{16 \mathrm{k}^{2}-1}\left[\mathrm{~J}_{\mathrm{k}}\left(\sqrt{2} \mathrm{nd} \tau_{\mathrm{r}}\right)\right]^{2}
$$

where $\mathrm{d}$ is the dipolar coupling constant and $\mathrm{n}$ is the total number of rotor periods over which the experiment is performed.

Figure 5.15 shows a variation of the REDOR experiment suitable for use with ${ }^{27} \mathrm{Al}-$ to- ${ }^{29} \mathrm{Si} \mathrm{CP}$. The train of $180^{\circ}$ pulses was applied to the detected spins based an experiment which was designed to minimize resonance offsets. ${ }^{202}$ Although offset effects 
were not a major concern in our case, this approach meant that only one $180^{\circ}$ pulse needed to be applied in the ${ }^{27} \mathrm{Al}$ channel. This differs from other sequences which used REDOR to measure distances between quadrupolar and spin-1/2 nuclei. ${ }^{134}$

An important aspect of the pulse sequence of Figure 5.15 is the fact that the ${ }^{29} \mathrm{Si}$ magnetization is generated by cross polarization from ${ }^{27} \mathrm{Al}$. This does more than just enhance the sensitivity of the ${ }^{29} \mathrm{Si}$ signal. If a $90^{\circ}$ pulse were directly applied to the ${ }^{29} \mathrm{Si}$ instead, the REDOR curve would have to be modified to account for that fact that some ${ }^{29} \mathrm{Si}$ nuclei would be dipolar-coupled to ${ }^{27} \mathrm{Al}$ nuclei that were not in central-transition states. A selective $180^{\circ}$ pulse on the ${ }^{27} \mathrm{Al}$ would only influence those that were in the central transition, leading to a decreased maximum of the $\Delta S / S_{0}$ curve. ${ }^{204}$ By using cross

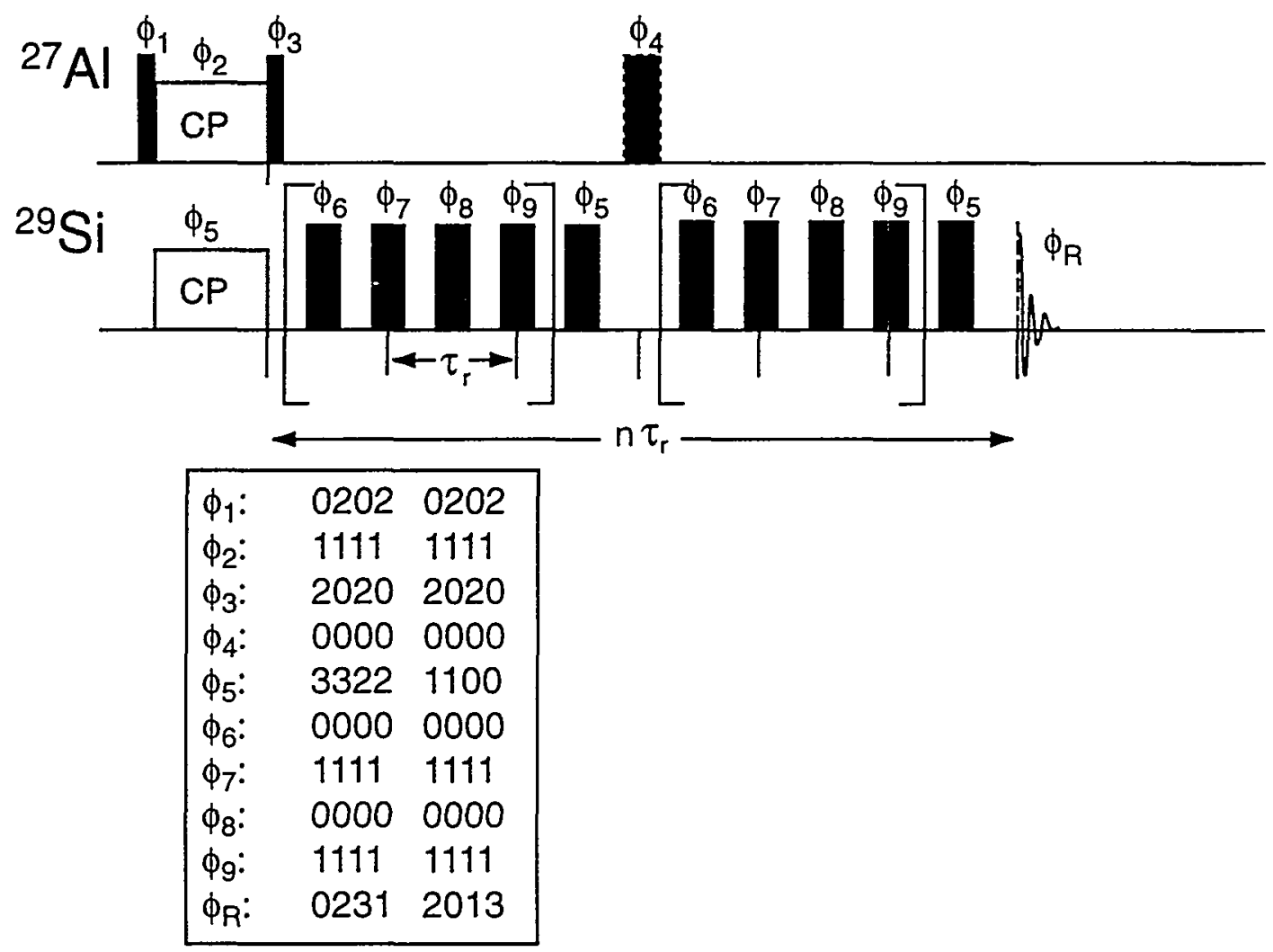

Figure 5.15 - A version of the REDOR experiment which can be used with cross polarization from quadrupolar nuclei. The bracketed parts of the sequence can be repeated, allowing the experiment to be performed over $n=6+4 N$ cycles where $N=0,1,2$, etc. XY-4 or XY-8 phase cycling is used for the bracketed pulses. ${ }^{202}$ The ${ }^{27} \mathrm{Al}$ spins are used as a magnetization source, and a flipback pulse ${ }^{203}$ restores their magnetization to along the z-axis. Two data sets are recorded: one with and one without the $180^{\circ}$ pulse on the aluminum. 
polarization in the sudden regime (where the eigenstates of the ${ }^{27} \mathrm{Al}$ do not change), one pre-selects only those ${ }^{29} \mathrm{Si}$ sites which are coupled to central-transition ${ }^{27} \mathrm{Al}$ states. The REDOR experiment can then be treated, to a good ayproximation, as one between two spin- $1 / 2$ nuclei.

Note that the recently developed Rotational-Echo Adiabatic-Passage DOuble Resonance (REAPDOR) ${ }^{205,206}$ experiment takes the opposite approach and attempts to observe the dipolar dephasing from all of the quadrupolar states. This is accomplished by using the interplay between an applied rf field and sample spinning in the adiabatic regime (see Section 3.2). In place of the $180^{\circ}$ pulse on the quadrupolar channel in a REDOR experiment, REAPDOR uses intense if irradiation to alter the quantum states of the quadrupolar nuclei, preventing dipolar refocusing by the pulses on the spin-1/2 channel. A predicted difference signal with two adjustable parameters (the dipolar-coupling constant and the fraction of spins which undergo the adiabatic passage) can then be calculated and compared with experiments.

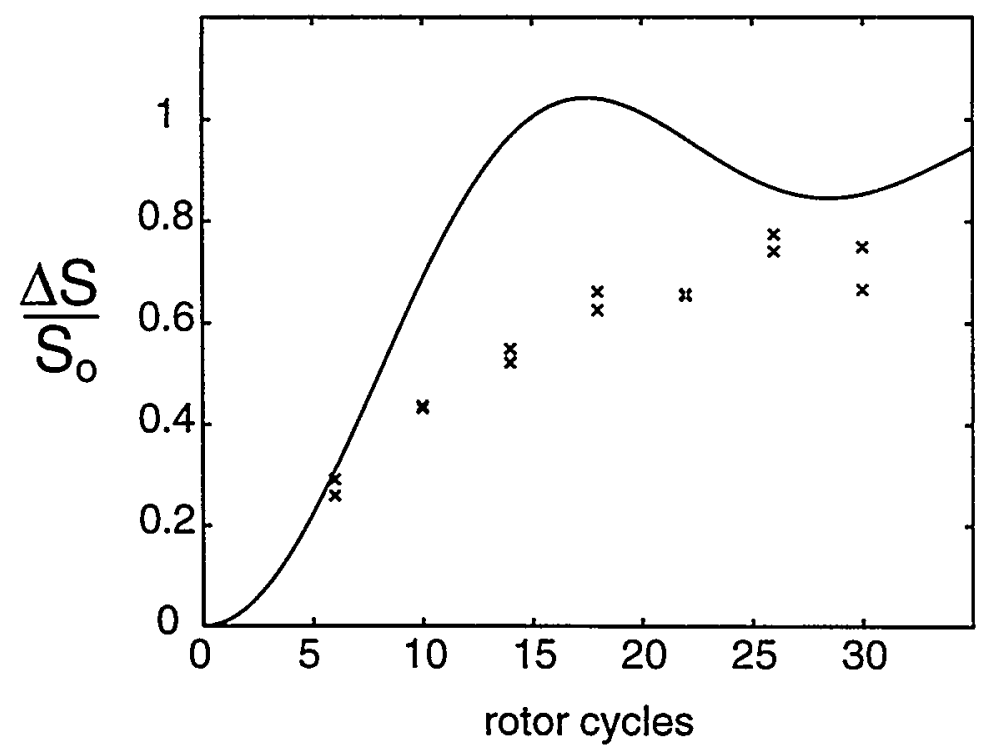

Figure $5.16-{ }^{27} \mathrm{Al} /{ }^{29} \mathrm{Si}$ REDOR of the T20 site in low albite. The $x$ 's represent experimental data points (from two sets of experiments) acquired using the pulse sequence of Figure 5.15, with a spinning speed of $2.2 \mathrm{kHz}, \mathrm{a}^{27} \mathrm{Al} 180^{\circ}$ pulse length of $33 \mu \mathrm{s}$, and a ${ }^{29} \mathrm{Si} 180^{\circ}$ pulse length of $52 \mu \mathrm{s}$. Due to $\mathrm{T}_{2}$ relaxation, the experiment could not be performed over more than 30 rotor cycles. The solid curve represents a simulation of the REDOR signal obtained using Equation (5.15) (truncated at $k=10$ ) with $d=210 \mathrm{~Hz}$. 
Figure 5.16 shows the results of REDOR applied to the T2O site in low albite using ${ }^{27} \mathrm{Al}-$ to $^{29} \mathrm{Si} \mathrm{CP}$. To a good approximation, this ${ }^{29} \mathrm{Si}$ site should see only one aluminum (with a coupling constant of $210 \mathrm{~Hz}$ ). Although the first experimental data point falls along the predicted curve, subsequent points due not. However, the theory of REDOR was developed assuming that the $180^{\circ}$ pulses were ideal (i.e. - of negligible length). Each $180^{\circ}$ pulse in our experiment was $11 \%$ of the length of the rotor cycle; this was the minimum pulse length achievable with a $100 \mathrm{~W}$ amplifier on our large coil probe. Ignoring the effects of dipolar coupling during the pulse is probably not valid in this case, and either the theory or experiment should be adjusted accordingly.

Other researchers have also used REDOR with quadrupolar nuclei. In many cases, the results were not quantitative, but this is to be expected because the approximation of isolated spin pairs was not valid. ${ }^{127,134,143,203,207}$ In cases where there were effectively isolated spin pairs, ${ }^{204,208}$ reasonable values for the dipolar-coupling constants were extracted (though no independent $\mathrm{X}$-ray confirmation was possible), but only points at the beginning of the REDOR curve (before $\Delta S / S_{0}$ reached a value of 0.5 ) were examined. 


\section{Chapter 6: High-Resolution Heteronuclear Correlation between Quadrupolar and Spin-1/2 Nuclei using Multiple-Quantum Magic-Angle Spinning}

In Chapters 3 and 4, the issues involved in cross polarization from quadrupolar nuclei were discussed, and in Chapter 5 several applications were demonstrated. In all of those applications, however, the quadrupolar nucleus was not directly observed. In this chapter, recent advances in the high-resolution spectroscopy of quadrupolar nuclei will be reviewed. Then a new technique that combines cross polarization from quadrupolar nuclei with the Multiple-Quantum Magic-Angle Spinning technique will be introduced. This new technique is capable of producing high-resolution heteronuclear correlation spectra in which one of the dimensions is from a half-integer quadrupolar nucleus.

\subsection{Methods for Obtaining High-Resolution Spectra of Quadrupolar Nuclei}

Although magic-angle spinning leads to high-resolution spectra for spin-1/2 nuclei, it fails to fully remove the second-order quadrupolar anisotropy. Consequently, MAS spectra of half-integer quadrupolar nuclei generally contain broad asymmetric peaks (see Section 1.4). The shapes of such peaks contain structural information (via the $\eta$ and $\mathrm{C}_{\mathrm{qcc}}$ parameters), but when multiple sites are present in a sample, the resolution is frequently poor. Fortunately, several techniques have been developed to produce highresolution spectra of quadrupolar nuclei.

In Section 1.2.5 it was shown that the $(+\mathrm{m} \leftrightarrow-\mathrm{m})$ transitions of a quadrupolar nucleus are unaffected to first order by the quadrupolar Hamiltonian. When second-order perturbation theory is applied, the frequencies of these transitions are affected in an orientation-dependent manner. For a sample spun at an angle $\theta$ with respect to the static field, the $(+\mathrm{m} \leftrightarrow-\mathrm{m})$ transition frequency of an individual crystallite is given by (see Equation (1.114)) 


$$
\begin{aligned}
\omega_{+m \leftrightarrow-m}^{(2 \mathrm{Q})}=\frac{\mathrm{C}_{\mathrm{qcc}}^{2}}{\omega_{\mathrm{L}}}\left\{\mathrm{A}_{0} \mathrm{C}_{0}(\mathrm{~S}, \mathrm{~m})\right. & +\mathrm{A}_{2}\left(\alpha^{\mathrm{Q}}, \beta^{\mathrm{Q}}\right) \mathrm{C}_{2}(\mathrm{~S}, \mathrm{~m}) \mathrm{P}_{2}(\cos \theta) \\
& \left.+\mathrm{A}_{4}\left(\alpha^{\mathrm{Q}}, \beta^{\mathrm{Q}}\right) \mathrm{C}_{4}(\mathrm{~S}, \mathrm{~m}) \mathrm{P}_{4}(\cos \theta)\right\}
\end{aligned}
$$

in the fast-spinning limit where $\alpha^{\mathrm{Q}}$ and $\beta^{\mathrm{Q}}$ are the Euler angles that define the orientation of the crystallite with respect to the axis of rotation; $\mathrm{P}_{2}$ and $\mathrm{P}_{4}$ are Legendre polynomials; $S$ is the spin-quantum number of the quadrupolar nucleus; and the $\left\{C_{n}(S, m)\right\}$ are scaling coefficients that depend on which $(+\mathrm{m} \leftrightarrow-\mathrm{m})$ coherence is excited. To obtain narrow resonances from quadrupolar nuclei in powder samples, the second and third terms on the right-hand side of Equation (6.1) must be made to vanish for all values of $\alpha^{\mathrm{Q}}$ and $\beta^{\mathrm{Q}}$. This can be accomplished either by manipulation of the physical space (using sample reorientation) or by manipulation of the spin space (using rf pulses).

\subsubsection{DOuble Rotation (DOR)}

The technique of DOuble Rotation (DOR) eliminates the second-order quadrupolar anisotropy of the central transition by spinning the sample about two axes simultaneously. One axis is at an angle of $54.74^{\circ}$ (the magic angle) with respect to the $\mathrm{B}_{0}$ field, and the second axis is at $30.56^{\circ}$ with respect to the magic-angle axis. Figure 6.1 shows a schematic of the rotor orientations in a DOR probe. Technical details of the probe design and other experimental considerations can be found in the Ph.D. thesis of K. T. Mueller ${ }^{10}$ while theoretical aspects are discussed in the thesis of B. Sun. ${ }^{209}$

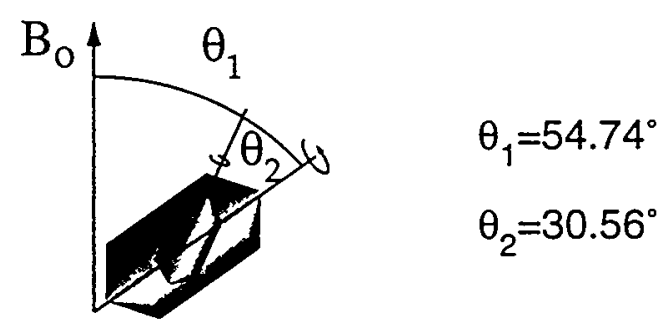

Figure 6.1 - Schematic of DOuble Rotation (DOR). An external rotor rotates at the magic angle while an internal rotor simultaneously spins at an angle of $30.56^{\circ}$ relative to the external rotor. 
To understand how DOR works, it is necessary to extend Equation (6.1) to the case of simultaneous rotation about two axes. This can be done by using an additional Wigner rotation matrix in a generalization of Equation (1.108)

$$
\begin{aligned}
& R_{1, m}=\sum_{n=-1 m^{\prime \prime}=-1 m^{\prime}=-1}^{1} \sum_{n, m}^{1}\left(\omega_{r 1} t+\phi_{r 1}, \theta_{1}, 0\right) D_{m^{\prime \prime}, n^{n}}^{(1)}\left(\omega_{r 2} t+\phi_{r 2}, \theta_{2}, 0\right) \\
& \times D_{m^{\prime}, m^{\prime \prime}}^{(1)}(\alpha, \beta, \gamma) \rho_{1, m^{\prime}} .
\end{aligned}
$$

Substituting Equation (6.2) into Equations (1.100) and (1.113) gives for $\mathrm{m}=1 / 2$ and rapid spinning ${ }^{10}$

$$
\begin{aligned}
\omega_{+\frac{1}{2} \leftrightarrow-\frac{1}{2}}^{(2 Q)}=\frac{C_{q c c}^{2}}{\omega_{L}}\left\{A_{0} C_{0}\left(S, \frac{1}{2}\right)\right. & +A_{2}\left(\alpha^{Q}, \beta^{Q}\right) C_{2}\left(S, \frac{1}{2}\right) P_{2}\left(\cos \theta_{1}\right) P_{2}\left(\cos \theta_{2}\right) \\
& \left.+A_{4}\left(\alpha^{Q}, \beta^{Q}\right) C_{4}\left(S, \frac{1}{2}\right) P_{4}\left(\cos \theta_{1}\right) P_{4}\left(\cos \theta_{2}\right)\right\}
\end{aligned}
$$

As in the single-axis case, the derivation is lengthy and is described elsewhere. ${ }^{10,209}$ However, the functional form of Equation (6.3) is quite similar to the single-axis case; the only difference is that the terms $P_{1}(\cos \theta)$ are replaced by products $P_{1}\left(\cos \theta_{1}\right) P_{1}\left(\cos \theta_{2}\right)$. The same substitution will also be made for Legendre polynomials appearing in other frequency expressions (e.g. - the chemical-shielding and dipolar anisotropies).

It is now clear how to eliminate the quadrupolar anisotropy by DOR. All that must be done is to choose $\theta_{1}$ and $\theta_{2}$ such that one angle is a zero of the second-order Legendre polynomial (the magic angle) and the other is a zero of the fourth-order Legendre polynomial (either $30.56^{\circ}$ or $70.12^{\circ}$ ). Then all the anisotropic terms will disappear simultaneously. For technical reasons (such as filling factor and sensitivity) the combination of angles shown in Figure 6.1 is most suitable for DOR experiments. ${ }^{210}$

In addition to eliminating the second-order quadrupolar broadening, DOR can, in principle, also average out the dipolar couplings and the chemical-shielding anisotropy since one of the rotors is spun at the magic angle. In practice, however, averaging of any of these anisotropies by DOR is incomplete since the outer rotor can only be spun at slow speeds (about $1 \mathrm{kHz}$ ). Thus, DOR spectra typically show a multitude of sidebands, ${ }^{211}$ 
which can make it difficult to identify the isotropic peaks from examination of a single spectrum. A comparison of DOR spectra recorded at different speeds, however, permits one to distinguish isotropic peaks from sidebands since the isotropic peaks will not shift their positions as a function of spinning speed.

Since DOR at least partially averages out dipolar couplings, it works well for highly abundant quadrupolar nuclei. It also works well for samples with short $T_{1}$ times (in contrast to DAS) because the averaging process is continuous. That same continuity is also a disadvantage, however, because it essentially limits DOR to being a onedimensional technique. Methods which average out the quadrupolar interaction sequentially are inherently more versatile since they permit a two-dimensional correlation of the completely averaged (isotropic) quadrupolar spectrum with an incompletely averaged (anisotropic) quadrupolar spectrum. Two such methods will be discussed below.

\subsubsection{Dynamic-Angle Spinning (DAS)}

Dynamic-Angle Spinning (DAS) also involves spatial manipulation of the sample to eliminate the quadrupolar anisotropy of the central transition. A schematic of a basic DAS experiment is shown in Figure 6.2. Many variants of this sequence exist and are discussed extensively elsewhere. ${ }^{11}$ In this section, we will not be concerned with the modes of data acquisition but will just illustrate the principle behind the experiment. Some of these data-acquisition issues are relevant for MQMAS, however, and will be discussed in Section 6.1.3.

In the DAS experiment, the sample is spun about an axis oriented at $\theta_{1}$ degrees with respect to the static field and magnetization evolves for a time $t_{1} /(k+1)$. A $90^{\circ}$ pulse stores one component of the evolving magnetization along the $\mathrm{z}$-axis while the axis of sample rotation is quickly reoriented to the angle $\theta_{2}$. Another $90^{\circ}$ pulse returns the magnetization to the transverse plane, and the magnetization now evolves at the second angle for a time $\mathrm{kt}_{1} /(\mathrm{k}+1)$. The angles of the two axes and the amount of time spent at each angle are chosen such that the anisotropic quadrupolar evolution of the central transition at the first angle is cancelled out by the evolution at the second angle, leading to the formation of an isotropic echo. Mathematically, these can be derived as follows. The signal at the time $t_{1}$ can be calculated from Equation (6.1) 


$$
\begin{aligned}
\omega_{+\frac{1}{2} \leftrightarrow-\frac{1}{2}}^{(2 Q)}\left(\theta_{1}\right) & \cdot \frac{\mathrm{t}_{1}}{\mathrm{k}+1}+\omega_{+\frac{1}{2} \leftrightarrow \frac{1}{2}}^{(2 \mathrm{Q})}\left(\theta_{2}\right) \cdot \frac{\mathrm{kt}_{1}}{\mathrm{k}+1} \\
& =\frac{\mathrm{C}_{\mathrm{qcc}}^{2}}{\omega_{\mathrm{L}}}\left\{\mathrm{A}_{0} \mathrm{C}_{0}\left(\mathrm{~S}, \frac{1}{2}\right) \cdot \mathrm{t}_{1}\right. \\
& +\mathrm{A}_{2}(\alpha, \beta) \mathrm{C}_{2}\left(\mathrm{~S}, \frac{1}{2}\right)\left[\mathrm{P}_{2}\left(\cos \theta_{1}\right)+\mathrm{kP}_{2}\left(\cos \theta_{2}\right)\right] \cdot \frac{\mathrm{t}_{1}}{\mathrm{k}+1} \\
& \left.+\mathrm{A}_{4}(\alpha, \beta) \mathrm{C}_{4}\left(\mathrm{~S}, \frac{1}{2}\right)\left[\mathrm{P}_{4}\left(\cos \theta_{1}\right)+\mathrm{kP}_{4}\left(\cos \theta_{2}\right)\right] \cdot \frac{\mathrm{t}_{1}}{\mathrm{k}+1}\right\}
\end{aligned}
$$

To cancel out the orientation-dependent terms $\mathrm{A}_{2}\left(\alpha^{\mathrm{Q}}, \beta^{\mathrm{Q}}\right)$ and $\mathrm{A}_{4}\left(\alpha^{\mathrm{Q}}, \beta^{\mathrm{Q}}\right)$ in Equation (6.4), the two angles of rotation and the time ratio $k$ must be chosen such that

$$
\begin{aligned}
& \mathrm{P}_{2}\left(\cos \theta_{1}\right)=-\mathrm{k} \cdot \mathrm{P}_{2}\left(\cos \theta_{2}\right) \\
& \mathrm{P}_{4}\left(\cos \theta_{1}\right)=-\mathrm{k} \cdot \mathrm{P}_{4}\left(\cos \theta_{2}\right)
\end{aligned}
$$
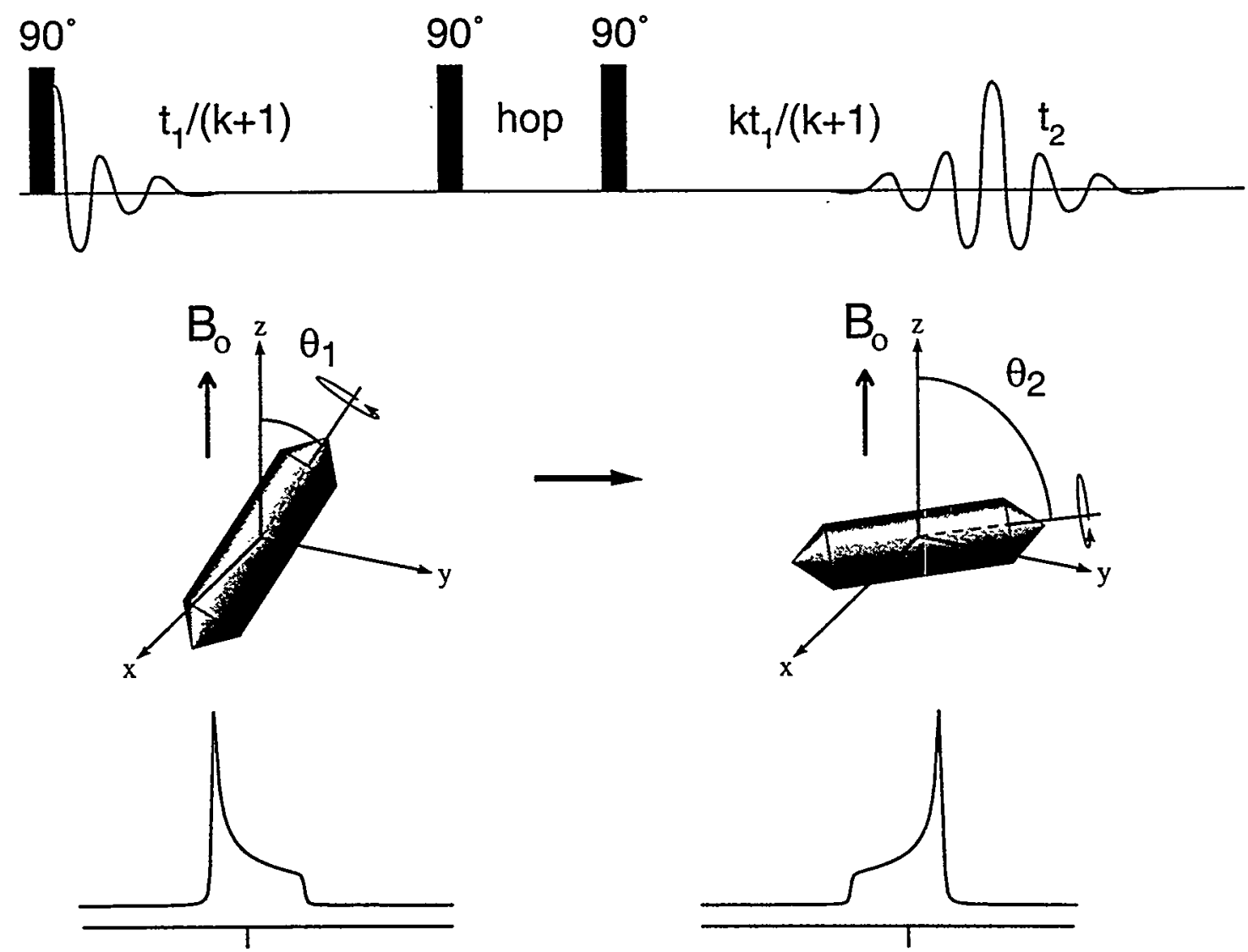

Figure 6.2 - Schematic of DAS experiment showing the timing of the rf pulses, the reorientation of the rotor axis, and the anisotropic lineshapes at each angle. 
Then Equation (6.4) simplifies to

$$
\omega_{+\frac{1}{2} \leftrightarrow-\frac{1}{2}}^{(2 Q)}\left(\theta_{1}\right) \cdot \frac{t_{1}}{k+1}+\omega_{+\frac{1}{2} \leftrightarrow-\frac{1}{2}}^{(2 Q)}\left(\theta_{2}\right) \cdot \frac{k t_{1}}{k+1}=\frac{C_{q c c}^{2}}{\omega_{L}}\left\{A_{0} C_{0}\left(S, \frac{1}{2}\right) \cdot t_{1}\right\}
$$

which is a purely isotropic frequency. The indirect $\left(\omega_{1}\right)$ dimension of a DAS experiment will, therefore, show peaks at positions given by (in ppm)

$$
\begin{aligned}
\delta_{\text {iso }}^{\text {DAS }} & =\delta_{\text {iso }}^{(C S)}+\frac{10^{6}}{\omega_{L}} \cdot \frac{C_{q c c}^{2}}{\omega_{L}}\left\{A_{0} C_{0}\left(S, \frac{1}{2}\right)\right\} \\
& =\delta_{\text {iso }}^{(C S)}+\frac{10^{6}}{\omega_{L}} \cdot \frac{C_{q c c}^{2}}{\omega_{L}}\left\{\frac{-3\left[S(S+1)-\frac{3}{4}\right]}{40 S^{2}(2 S-1)^{2}} \cdot\left(1+\frac{\eta^{2}}{3}\right)\right\} \\
& =\delta_{i s o}^{(C S)}+\delta_{i s o}^{(2 Q)} .
\end{aligned}
$$

The first term on the right-hand side of Equation (6.7) is the isotropic chemical shift. The second term on the right-hand side of Equation (6.7) is often called the second-order isotropic quadrupolar shift. It can be extracted from DAS experiments or, with less accuracy, from fits of quadrupolar powder lineshapes. Note that this shift is inversely proportional to the Larmor frequency; DAS experiments performed at different fields will, therefore, have different isotropic shifts even when these shifts are expressed in ppm. Many combinations of $\theta_{1}, \theta_{2}$, and $k$ satisfy Equations (6.5) and are plotted in Figure 6.3. Note that the magic angle is not one of the possible solutions. For technical reasons related to sideband positions, only two solutions are practical for DAS experiments. "One is the set $\left(\theta_{1}=37.38^{\circ}, \theta_{2}=79.19^{\circ}, \mathrm{k}=1\right)$ and the other is the set $\left(\theta_{1}=63.43^{\circ}, \theta_{2}=0.00^{\circ}\right.$, $k=5$ ). The latter set is compelling because of the possibility of correlating isotropic chemical shifts with full, static powder lineshapes (obtained by spinning about $0.00^{\circ}$ ), but specialized probe designs, which are not currently commercially available, are required to irradiate samples at $0.00^{\circ}$.

Although the $\mathrm{P}_{2}(\cos \theta)$ contribution to the quadrupolar anisotropy is refocused in the $\omega_{1}$ dimension of a DAS experiment (see Equation (6.5)), the homonuclear dipolar couplings, which also have a $\mathrm{P}_{2}(\cos \theta)$ spatial dependence, will not be refocused. This is 
due to the fact that the $\mathrm{z}$-filter does not store all of the relevant spin operators for dipolar coupling, prohibiting complete reversal of the dipolar Hamiltonian in the second half of the experiment. ${ }^{122}$ Thus, DAS will not work well for samples with strong dipolar couplings. DAS also can only be performed on samples with $T_{1}$ relaxation times that exceed the time it takes to reorient the sample (typically 30-50 ms). However, many quadrupolar nuclei (such as ${ }^{17} \mathrm{O}$ ) do have sufficiently long $\mathrm{T}_{1}$ 's to permit DAS experiments to be performed.

An advantage of DAS over DOR is its inherent two-dimensional nature which permits the correlation of a high-resolution isotropic spectrum with site-specific, anisotropic powder patterns. From such powder patterns, quadrupolar parameters can be determined. Figure 6.4 shows an ${ }^{17} \mathrm{O}$ DAS spectrum of $\mathrm{AlPO}_{4}-5$ acquired with $\mathrm{k}=5$ and sheared (see Section 1.5.2). Two oxygen sites are present in the sample, and their quadrupolar parameters were extracted from lineshape simulations.

Many variations and extensions of the DAS experiment have been developed and an in-depth discussion of most of them can be found in the thesis of J. H. Baltisberger. ${ }^{11}$

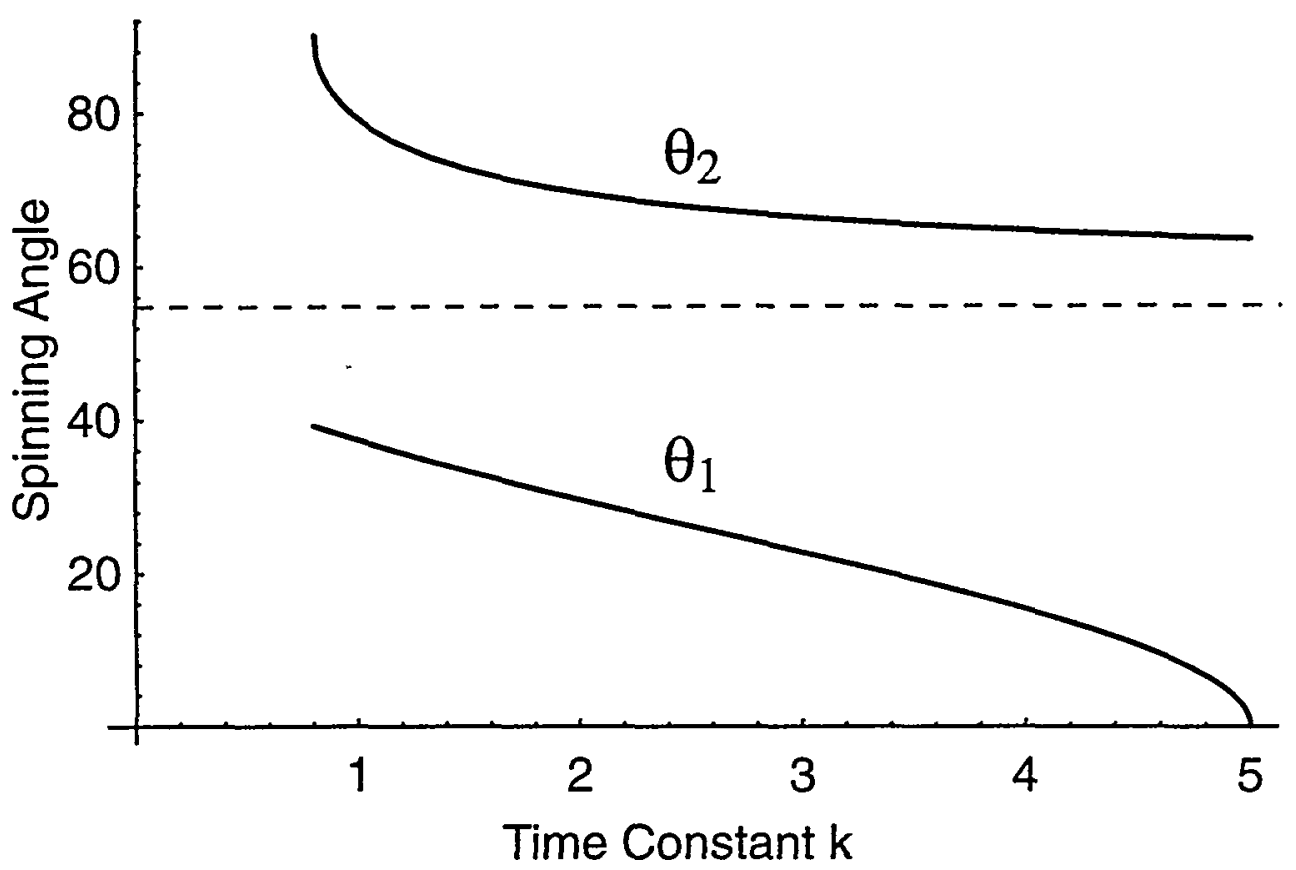

Figure 6.3 - Angle pairs which are solutions to Equations (6.5). For each value of $k$ (which is the ratio of the time spent at $\theta_{2}$ to the time spent at $\theta_{1}$ ), the corresponding values of $\theta_{1}$ and $\theta_{2}$ can be read off. Note that the magic angle (represented by a dotted line) is not one of the solutions to Equations (6.5). 


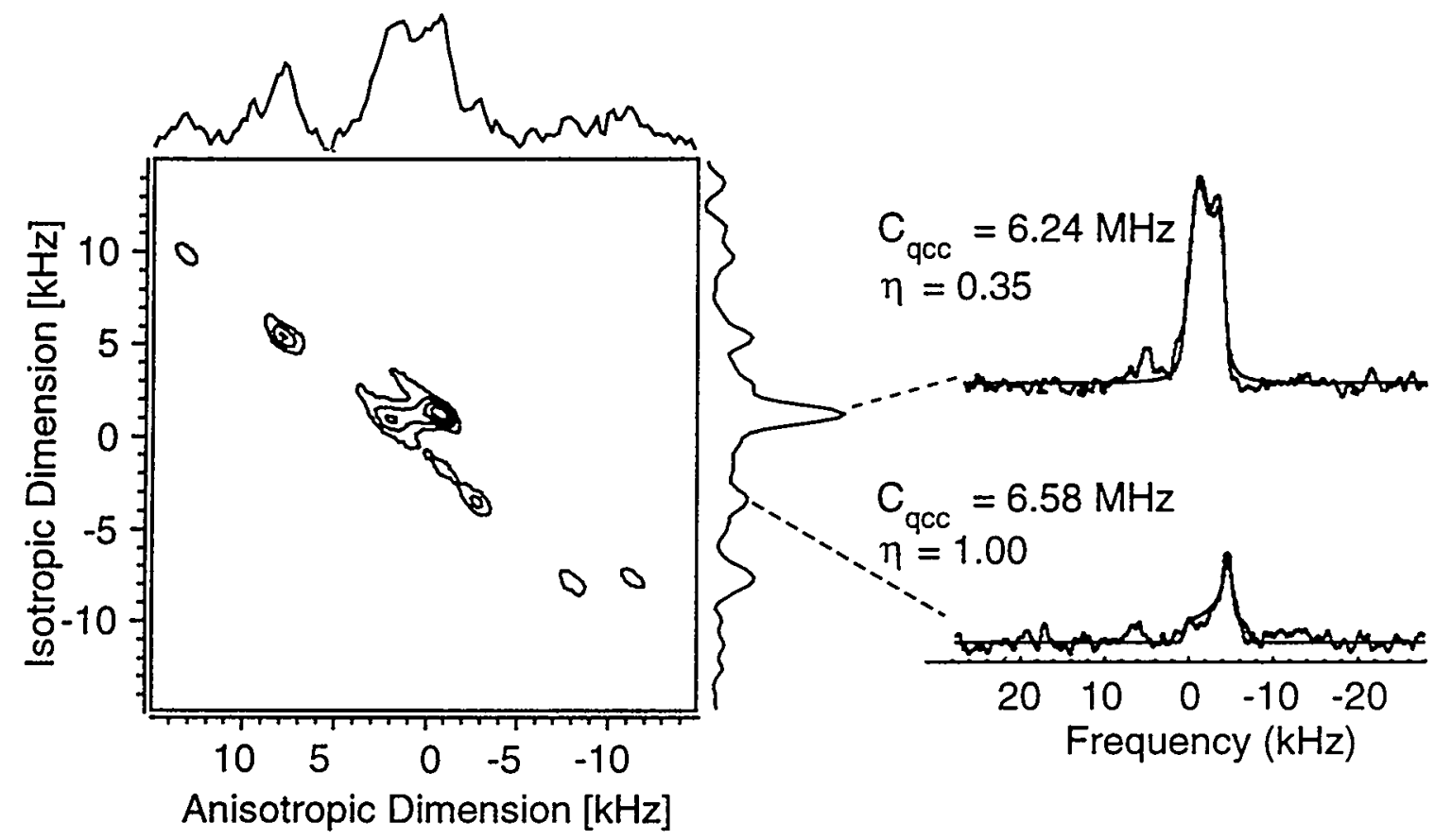

Figure $6.4-{ }^{17} \mathrm{O}$ DAS spectrum of $\mathrm{AlPO}_{4}-5$ showing the two sites and fits of the anisotropic lineshapes for $\theta_{2}=63.43^{\circ}$.

\subsubsection{Multiple-Quantum Magic-Angle Spinning (MQMAS)}

While DOR and DAS use spatial manipulation of the sample to achieve highresolution spectra of quadrupolar nuclei, the Multiple-Quantum Magic-Angle Spinning (MQMAS) technique achieves high resolution by manipulating the $\left\{\mathrm{C}_{\mathrm{n}}(\mathrm{S}, \mathrm{m})\right\}$ terms of Equation (6.1) while spinning only at the magic angle. Like DAS, MQMAS does its averaging sequentially. First, a multiple-quantum coherence connecting the states $+m_{1}$ and $-m_{1}$ is excited and allowed to evolve for a time period $t_{1} /(k+1)$. This is then converted to a single-quantum coherence between the $+1 / 2$ and $-1 / 2$ states. The singlequantum coherence will evolve, and an isotropic echo will be generated at a time $\mathrm{kt}_{1} /(\mathrm{k}+1)$ after the conversion.

Several different pulse schemes have been used for the excitation and conversion of the coherences. The original experiment was performed using two selective $90^{\circ}$ pulses with a short delay (tens to hundreds of $\mu \mathrm{s}$ ) between them for excitation. The first pulse generated \pm 1 quantum coherences and the second pulse converted them to multiplequantum coherences. ${ }^{42}$ Excitation of a multiple-quantum coherence is also possible with a 
single long pulse..$^{53.212}$ Although to first order weak rf-fields selectively excite only the central transition, higher-order treatments show that multiple-quantum coherences can also be excited to a limited extent. This has been described theoretically for the case of a single crystal. ${ }^{213,214}$ For on-resonance irradiation with $\left|\omega_{1 S} / \Omega^{(1 Q)}\right| \ll 1$, it can be shown that the effective nutation frequency of an n-quantum transition is of the $\operatorname{order}^{214,137}$

$$
\frac{\left(\omega_{1 S}\right)^{n}}{\left(\Omega^{(1 Q)}\right)^{n-1}}
$$

where $\Omega^{(1 Q)}$ is defined in Equation (3.5). Because this nutation frequency is small, very long pulse lengths are needed to generate appreciable amounts of multiple-quantum coherence. $^{214}$

Extension of the single-crystal treatment to a powder sample is not straightforward. ${ }^{121}$ This is due to the orientation dependence of $\Omega^{(1 Q)}$, which means that different crystallites will nutate with different frequencies even in a static sample. Furthermore, the above method of excitation only works for the case where $\omega_{1 \mathrm{~S}}$ is significantly larger than $\omega_{m \leftrightarrow-m}^{(2 Q)}$ for all values of $m,{ }^{214}$ and the scheme was predicted to fail entirely for powder samples. ${ }^{121}$

Nonetheless, brute-force application of longer pulses has met with some success. As expected, the excitation efficiency is highly dependent on crystallite orientation and quadrupolar parameters as well as rf-field strength and spinning speed. ${ }^{53,212}$ Optimal pulse lengths and strengths will, therefore, differ from sample to sample. The only general consensus that has been reached so far is that high values of the rf-field strength (hundreds of $\mathrm{kHz}$ ) are superior to low values ${ }^{43}$ although this does not preclude performing the MQMAS experiment with modest field strengths (see Figure 6.6).

The conversion of the multiple-quantum coherence to the single-quantum coherence is even less efficient than the excitation. ${ }^{212}$ Fortunately, even a highly inefficient conversion step will not significantly distort the anisotropic central-transition powder patterns recorded during $t_{2}$, provided the spinning speed exceeds the width of these patterns. ${ }^{43}$ The relative intensities of the different sites are distorted, however, and the intensities in the isotropic dimension cannot be used for quantitative purposes. At the 
time of this writing, no MQMAS pulse sequence reliably produces quantitative spectra; improvement of the excitation and conversion schemes is currently an active area of research.

Figure 6.5 shows a schematic of the single-pulse excitation version of the MQMAS experiment. As in the case of DAS, the conditions necessary to eliminate the anisotropy can be easily determined. The signal at the time $t_{1}$ can be calculated from Equation (6.1) as follows

$$
\begin{aligned}
\omega_{\mathrm{m}_{1} \leftrightarrow-\mathrm{m}_{1}}^{(2 \mathrm{Q})}(\theta) & \frac{\mathrm{t}_{1}}{\mathrm{k}+1}+\omega_{+\frac{1}{2} \leftrightarrow-\frac{1}{2}}^{(2 \mathrm{Q})}(\theta) \cdot \frac{\mathrm{kt}_{1}}{\mathrm{k}+1} \\
= & \frac{\mathrm{C}_{\mathrm{qcc}}^{2}}{\omega_{\mathrm{L}}}\left\{\mathrm{A}_{0}\left[\mathrm{C}_{0}\left(\mathrm{~S}, \mathrm{~m}_{1}\right)+\mathrm{kC}_{0}\left(\mathrm{~S}, \frac{1}{2}\right)\right] \cdot \frac{\mathrm{t}_{1}}{\mathrm{k}+1}\right. \\
& +\mathrm{A}_{2}(\alpha, \beta)\left[\mathrm{C}_{2}\left(\mathrm{~S}, \mathrm{~m}_{1}\right)+\mathrm{kC}_{2}\left(\mathrm{~S}, \frac{1}{2}\right)\right] \mathrm{P}_{2}(\cos \theta) \cdot \frac{\mathrm{t}_{1}}{\mathrm{k}+1} \\
& \left.+\mathrm{A}_{4}(\alpha, \beta)\left[\mathrm{C}_{4}\left(\mathrm{~S}, \mathrm{~m}_{1}\right)+\mathrm{kC}_{4}\left(S, \frac{1}{2}\right)\right] \mathrm{P}_{4}(\cos \theta) \cdot \frac{\mathrm{t}_{1}}{\mathrm{k}+1}\right\}
\end{aligned}
$$
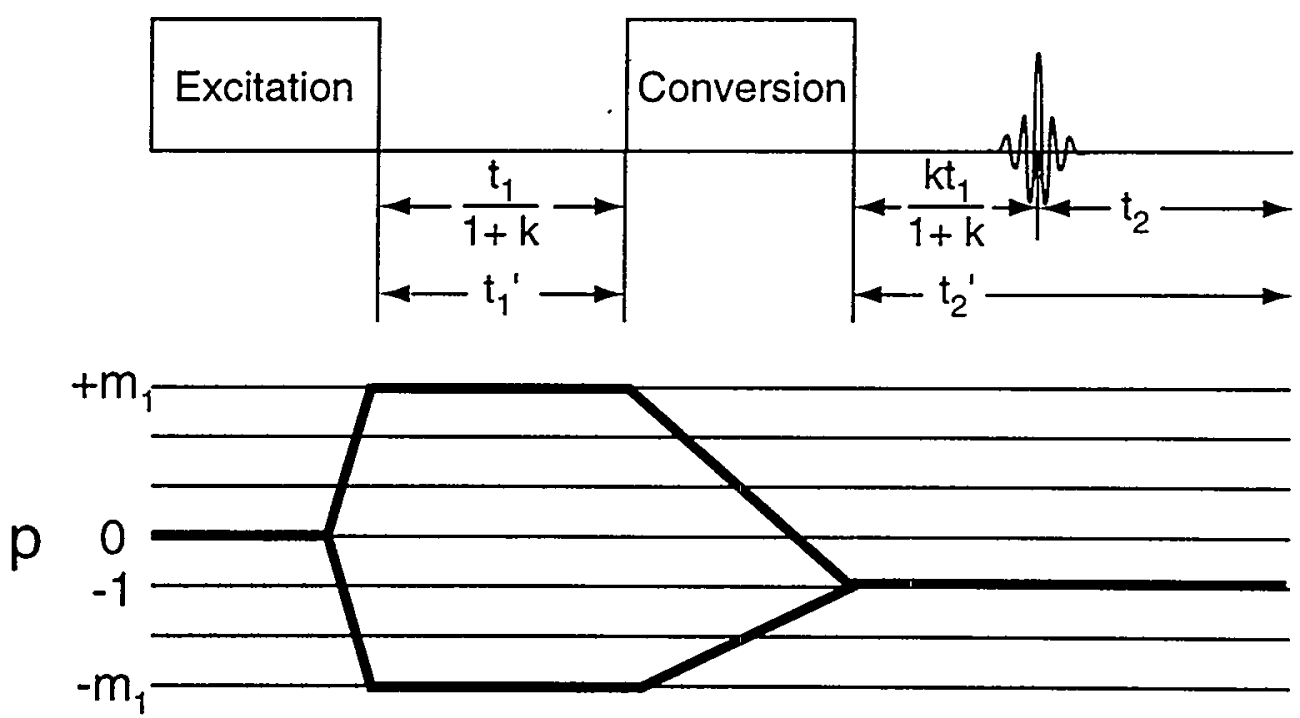

Figure 6.5 - Schematic of MQMAS experiment. In this chapter, the time $t_{1}$ is defined, for theoretical simplicity, as the time required for the formation of the isotropic echo. However, pure-phase spectra cannot be obtained using the times $\left(t_{1}, t_{2}\right)$ unless extra read pulses are added. ${ }^{215}$ In practice, it is easier to start the acquisition immediately after the reconversion pulse (using the times $t_{1}{ }^{\prime}$ and $t_{2}{ }^{\prime}$ ), and then shear the resulting twodimensional spectrum as described in the text. 
To eliminate the anisotropy, the last two terms on the right-hand side of Equation (6.9) must be set to zero. At the magic angle, $\mathrm{P}_{2}(\cos \theta)$ equals zero. To eliminate the remaining term, the only condition that must be met is

$$
\mathrm{C}_{4}\left(\mathrm{~S}, \mathrm{~m}_{1}\right)=-\mathrm{k} \cdot \mathrm{C}_{4}\left(\mathrm{~S}, \frac{1}{2}\right)
$$

\begin{tabular}{|c|c|c|c|c|}
\hline $\mathbf{S}$ & $\mathbf{m}$ & $\mathbf{C}_{\mathbf{0}}(\mathbf{S}, \mathbf{m})$ & $\mathbf{C}_{\mathbf{2}}(\mathbf{S}, \mathbf{m})$ & $\mathbf{C}_{\mathbf{4}}(\mathbf{S}, \mathbf{m})$ \\
\hline \hline$\frac{3}{2}$ & $\frac{1}{2}$ & 3 & 24 & 54 \\
\hline$\frac{3}{2}$ & $\frac{3}{2}$ & -9 & 0 & -42 \\
\hline$\frac{5}{2}$ & $\frac{1}{2}$ & 8 & 64 & 144 \\
\hline$\frac{5}{2}$ & $\frac{3}{2}$ & 6 & 120 & 228 \\
\hline$\frac{5}{2}$ & $\frac{5}{2}$ & -50 & -40 & -300 \\
\hline
\end{tabular}

Table 6.1 - Table of scaling coefficients used to describe the second-order quadrupolar frequency of a $+\mathrm{m} \leftrightarrow-\mathrm{m}$ coherence (see Equation $(6.1)^{42}$ ). The values were calculated according to the formulas $\mathrm{C}_{0}(\mathrm{~S}, \mathrm{~m})=2 \mathrm{~m}\left[\mathrm{~S}(\mathrm{~S}+1)-3 \mathrm{~m}^{2}\right], \quad \mathrm{C}_{2}(\mathrm{~S}, \mathrm{~m})=2 \mathrm{~m}\left[8 \mathrm{~S}(\mathrm{~S}+1)-12 \mathrm{~m}^{2}-3\right]$ and $\mathrm{C}_{4}(\mathrm{~S}, \mathrm{~m})=$ . $2 m\left[18 S(S+1)-34 m^{2}-5\right] .43$

Note that a similar type of experiment could be performed while spinning at one of the zeros of the fourth-order Legendre polynomial $\left(\theta=30.56^{\circ}\right.$ or $\left.\theta=70.12^{\circ}\right)$ and eliminating the quadrupolar anisotropy by choosing $\mathrm{k}$ according to

$$
\mathrm{C}_{2}\left(\mathrm{~S}, \mathrm{~m}_{1}\right)=-\mathrm{k} \cdot \mathrm{C}_{2}\left(\mathrm{~S}, \frac{1}{2}\right)
$$

This experiment is known as Multiple-Quantum Variable Angle Spinning and can be used to extract chemical-shift parameters. ${ }^{216}$ In this thesis, only the magic-angle version will be discussed, and values of $\mathrm{k}$ will be chosen according to Equation (6.10). The values of $\left\{C_{n}(S, m)\right\}$ for $S=3 / 2$ and $S=5 / 2$ are listed in Table 6.1; values for $S>5 / 2$ are tabulated elsewhere. ${ }^{42,43,216}$ For an MQMAS experiment on a spin-3/2 system, only one value of $k$ is 
possible $(\mathrm{k}=7 / 9)$. For higher values of $\mathrm{S}$, there is a choice of multiple-quantum coherences and, therefore, more than one possible value of $k$. For instance, for a spin-5/2 system, excitation of the triple-quantum coherence gives $k=-19 / 12$ while excitation of the fivequantum coherence gives $\mathrm{k}=-25 / 12$.

The isotropic second-order quadrupolar frequency that results from the MQMAS experiment is given (in ppm) by

$$
\begin{aligned}
\omega_{\mathrm{m}_{1} \leftrightarrow-\mathrm{m}_{1}}^{(2 \mathrm{Q})}(\theta) \cdot \frac{\mathrm{t}_{1}}{\mathrm{k}+1}+\omega_{+\frac{1}{2} \rightarrow-\frac{1}{2}}^{(2 \mathrm{Q})}(\theta) \cdot \frac{\mathrm{kt}_{1}}{\mathrm{k}+1} \\
\quad=\frac{\mathrm{C}_{\mathrm{qcc}}^{2}}{\omega_{\mathrm{L}}} \mathrm{A}_{0}\left[\mathrm{C}_{0}\left(\mathrm{~S}, \mathrm{~m}_{1}\right)+\mathrm{k} \mathrm{C}_{0}\left(\mathrm{~S}, \frac{1}{2}\right)\right] \cdot \frac{\mathrm{t}_{1}}{\mathrm{k}+1}
\end{aligned}
$$

A comparison of Equations (6.12) and (6.6) shows that the second-order isotropic quadrupolar shift in the MQMAS experiment differs from that in a normal MAS or DAS experiment. The isotropic chemical shift will also be scaled since the chemical shift of an $\mathrm{n}$-quantum coherence is $\mathrm{n}$ times that of a single-quantum coherence. For an MQMAS experiment, a weighted average of the two isotropic shifts can be calculated ${ }^{53}$

$$
2 \mathrm{~m} \cdot \delta_{\text {iso }}^{(\mathrm{CS})} \cdot \frac{\mathrm{t}_{1}}{\mathrm{k}+1}+\delta_{\text {iso }}^{(\mathrm{CS})} \cdot \frac{\mathrm{kt}_{1}}{\mathrm{k}+1}
$$

From Equations (6.7), (6.12), and (6.13), the observed shifts in the MQMAS experiment will, therefore, be given by

$$
\delta_{\text {iso }}^{\text {MQMAS }}=\left(\frac{2 \mathrm{~m}_{1}+\mathrm{k}}{\mathrm{k}+1}\right) \cdot \delta_{\text {iso }}^{(\mathrm{CS})}+\left[\frac{\mathrm{C}_{0}\left(\mathrm{~S}, \mathrm{~m}_{1}\right)+\mathrm{k} \mathrm{C}_{0}\left(\mathrm{~S}, \frac{1}{2}\right)}{(\mathrm{k}+1) \mathrm{C}_{0}\left(\mathrm{~S}, \frac{1}{2}\right)}\right] \cdot \delta_{\text {iso }}^{(2 \mathrm{Q})}
$$

where $\delta_{\text {iso }}^{(2 Q)}$ is the conventional "second-order quadrupolar shift" for the central transition (see Equation (6.7)) and $\mathrm{k}$ is given by Equation (6.10).

Figure 6.6 shows ${ }^{23} \mathrm{Na}$ 3Q/1Q MQMAS spectra of sodium pyrophosphate $\left(\mathrm{Na}_{4} \mathrm{P}_{2} \mathrm{O}_{7}\right)$ acquired using a single pulse for excitation and using hypercomplex shiftedecho detection (see Section 1.5.2). Five-radian pulses with lengths of $15.6 \mu \mathrm{s}$ (corresponding to $\omega_{1 S} /(2 \pi)=25.5 \mathrm{kHz}$ ) were used for both the excitation and 
reconversion. After a delay of $3.5 \mathrm{~ms}$, a softer $180^{\circ}$ pulse with a field strength of $15.6 \mathrm{kHz}$ was used to form an echo. The detection period $\left(t_{2}{ }^{\prime}\right)$ was begun immediately after this pulse. The first pulse was cycled in steps of $60^{\circ}$ to select the \pm 3 coherence transfer pathways and the third pulse was cycled in $45^{\circ}$ increments. The data were processed by constructing echo and anti-echo data sets according to Equations (1.129) and (1.130)..$^{53}$ The top (unsheared) spectrum was obtained by directly Fourier transforming both dimensions of both data sets (using Equation (1.140) where $f=k /(1+k)$ ), reversing the $\omega_{1}{ }^{\prime}$ dimension of the anti-echo data set, and summing the spectra. This leads to a spectrum in which the multiple-quantum spectrum along $\omega_{1}{ }^{\prime}$ is directly correlated with
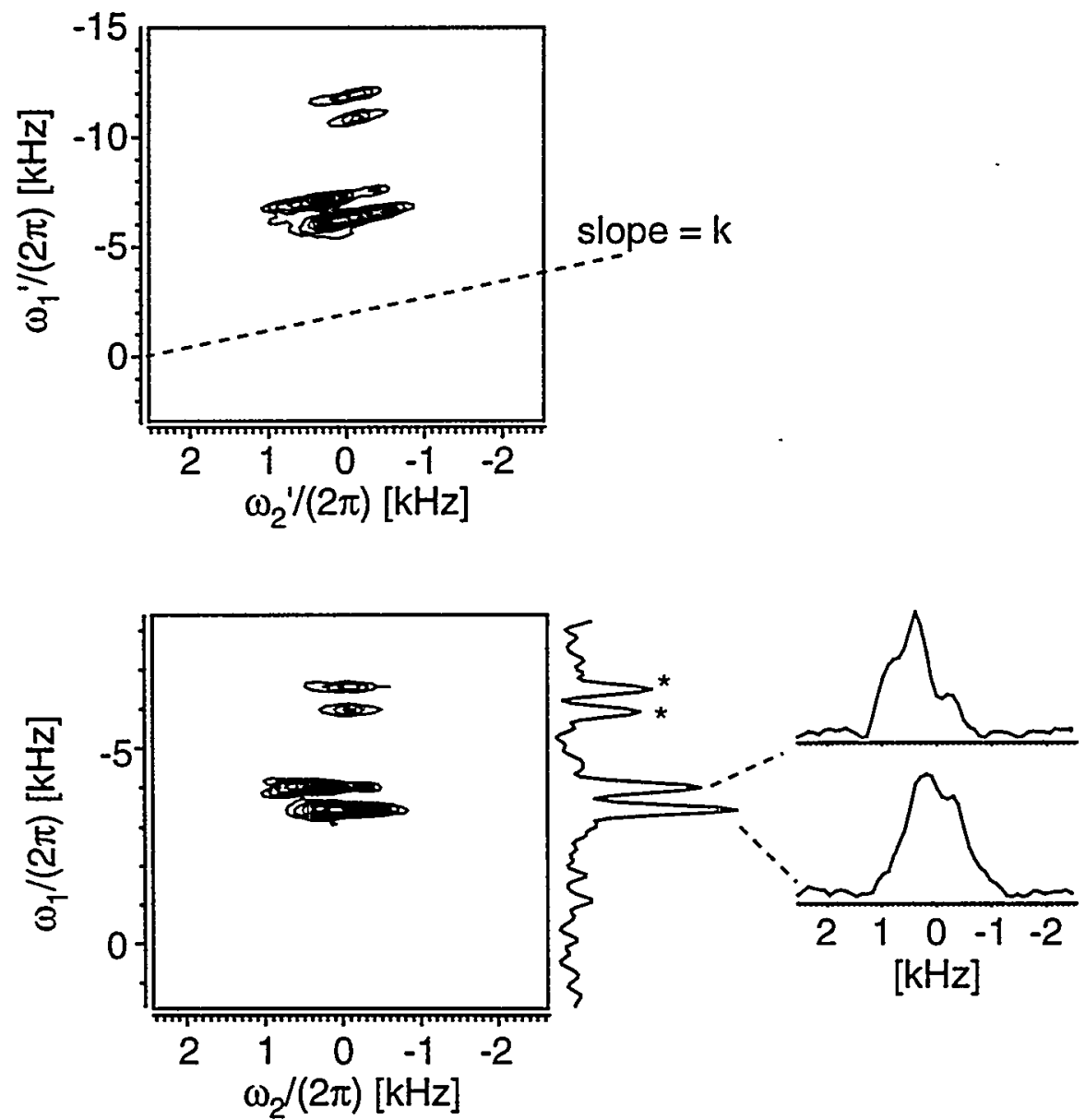

Figure 6.6 - ${ }^{23} \mathrm{Na}$ MQMAS spectrum of sodium pyrophosphate $\left(\mathrm{Na}_{4} \mathrm{P}_{2} \mathrm{O}_{7}\right)$. Both the unsheared and sheared 2D spectra are shown as well as the isotropic dimension and corresponding anisotropic powder patterns. Sixty hypercomplex pairs of $t_{1}$ slices with 96 scans in each were acquired with a $0.5 \mathrm{~s}$ recycle delay and a $30 \mu \mathrm{s}$ dwell time. Asterisks are used to denote spinning sidebands. 
the single-quantum MAS spectrum along $\omega_{2}{ }^{\prime}$. Because the anisotropic portions of each dimension are related, sharp diagonal peaks result. ${ }^{43}$ Site-specific, quadrupolar-broadened MAS lineshapes can be seen by taking skew projections along axes parallel to $\omega_{1}^{\prime}=k \omega_{2}^{\prime} \cdot 217$

It is often more convenient, however, to view the spectrum with the isotropic dimension along one axis and the anisotropic dimension along the other as was done for the chemical-shift interaction in Chapter 5 . One way to accomplish this is by shearing the spectrum (see Section 1.5.2). The lower spectrum in Figure 6.6 was obtained by Fourier transforming both data sets with respect to $t_{2}^{\prime}$ and then applying a phase correction of $e^{+i k \omega_{2}^{\prime} t_{1}{ }^{\prime}}$ to the echo spectrum and $e^{-i k \omega_{2} t_{1}{ }^{\prime}}$ to the anti-echo spectrum. The rest of the processing was the same as in the unsheared case. Because shearing effectively redefines the time dimensions, the spectral width of the $\omega_{1}$ dimension in the sheared spectrum will be a factor of $(\mathrm{k}+1)$ smaller than the $\omega_{1}{ }^{\prime}$ dimension in the unsheared spectrum, necessitating a rescaling of this axis. Direct isotropic/anisotropic correlation spectra can also be obtained by delaying the acquisition until the isotropic echo is formed ${ }^{215}$ although additional pulses will be needed to obtain pure-phase spectra (see Section 1.5.2).

The MQMAS technique has been applied to many samples in the two years since it was developed. It has several advantages over previous methods. Since it is performed at the magic angle, the dipolar couplings will be averaged out, and there is no restriction on $\mathrm{T}_{1}$ relaxation times. In addition, the experiment can be performed on conventional MAS equipment. However, the efficiency of the multiple-quantum excitation and conversion is strongly dependent on $\mathrm{C}_{\mathrm{qcc}}$ which makes obtaining signals with quantitative intensities difficult. Furthermore, excitation of high-order $(n>3)$ multiple-quantum coherences in a powder sample is difficult unless the quadrupolar coupling constants are small. A discussion of the advantages and disadvantages of MQMAS relative to DAS is found in the literature. ${ }^{216}$

We were interested in seeing if the MQMAS experiment could be combined with cross polarization (using conditions similar to those discussed in Chapter 4) to yield a new technique for examining heteronuclear distances in solids. Before discussing our technique, it is useful to review previous heteronuclear correlation experiments in solids. 


\subsection{Heteronuclear Correlation and Quadrupolar Nuclei}

\subsubsection{MAS- and DAS-Based Techniques}

HETeronuclear CORrelation (HETCOR) experiments were first used in the solution state to probe through-bond connectivities via J-couplings. ${ }^{4,218}$ They were later applied to solids under both static and MAS conditions. ${ }^{219,220,221}$ In the solid-state cases, the experiments rely on residual dipolar couplings. The connectivities that are measured are therefore "through-space" rather than "through-bond," and the results are inherently qualitative. Nonetheless, solid-state HETCOR experiments have provided useful information about proximities of different chemical species in many systems. They have been used to characterize surface-adsorbate interactions and to assign peaks in complicated spectra.

The most basic version of the HETCOR experiment in solids is simply a twodimensional extension of cross polarization in which the pulse that generates the transverse magnetization is separated from the Hartmann-Hahn matched pulses by a time period $t_{1}$ which frequency-labels the source spins (see Figure 6.7). ${ }^{221}$ The resulting twodimensional spectrum will then show cross peaks between sites in the MAS spectrum of the first nucleus and sites in the MAS spectrum of the second nucleus that are spatially near enough for polarization transfer to occur between them. More complicated pulse sequences exist for cases in which homonuclear decoupling is necessary. In addition, there are variants which utilize a TEDOR-type coherence transfer step in place of the cross polarization. ${ }^{134,207}$

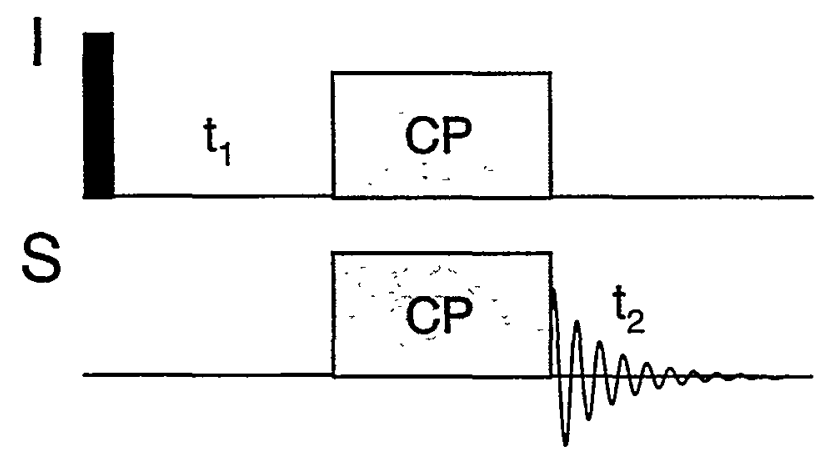

Figure 6.7 - Basic pulse sequence for HETCOR experiment. 

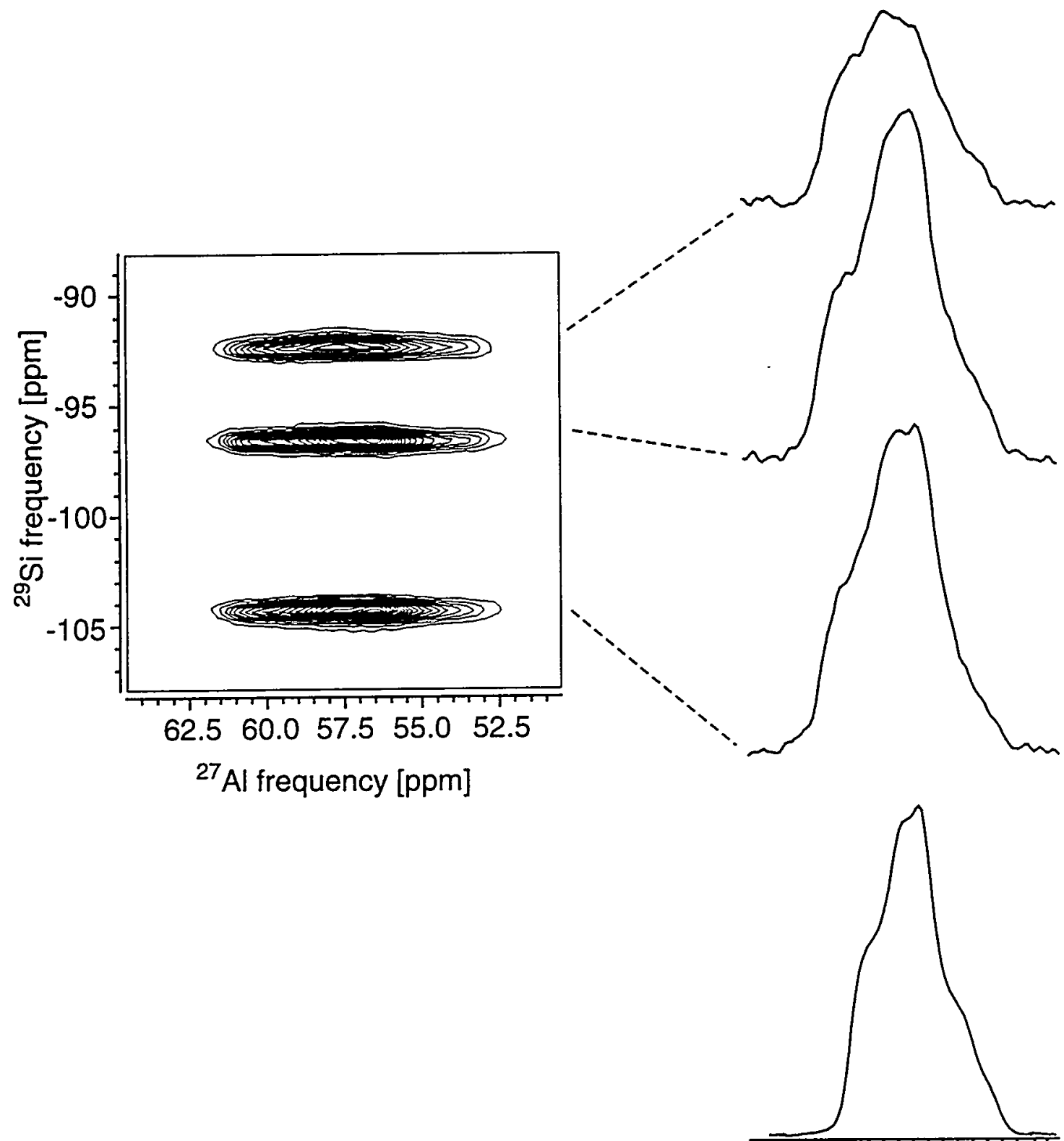

$62.5 \quad 60.057 .555 .0$

Aluminum MAS

Figure $6.8-{ }^{27} \mathrm{Al} /{ }^{29} \mathrm{Si}$ MAS HETCOR spectrum of low albite. Both a two-dimensional contour plot as well as individual slices along the $\omega_{2}$ dimension are shown. For comparison, the 1D MAS spectrum of ${ }^{27} \mathrm{Al}$ is also shown. Note that the ${ }^{27} \mathrm{Al}$ lineshape correlated with the silicon site with two aluminum nearest neighbors is distorted. 
When the MAS spectrum of a quadrupolar nucleus is sufficiently well-resolved, experiments which correlate the central transition of a quadrupolar nucleus with a spin-1/2 nucleus can also be performed. ${ }^{134,142,143,207,222}$ Figure 6.8 shows an ${ }^{27} \mathrm{Al} /{ }^{29} \mathrm{Si}$ MAS HETCOR spectrum of low albite performed using ${ }^{27} \mathrm{Al}-$ to- $^{29} \mathrm{Si} \mathrm{CP}$ with a linear amplitude ramp on the ${ }^{29} \mathrm{Si}$ (see Figure 4.16). The proximity of the aluminum atoms to each type of silicon site is evidenced by the presence of three cross peaks, although the peak intensities, as in the one-dimensional $\mathrm{CP}$ case, are not quantitative.

If only one site of a given type is present, however, it is not necessary to perform a two-dimensional experiment since the same qualitative information is obtainable from a one-dimensional cross-polarization experiment. The HETCOR experiment is most useful for samples which have multiple sites, but spectra of quadrupolar nuclei are often not well-resolved under MAS. To extend the applicability of heteronuclear-correlation techniques to more samples containing quadrupolar nuclei, a high-resolution heteronuclear correlation technique which combines Dynamic-Angle Spinning (DAS) with cross polarization was developed by Jarvie et al. ${ }^{223}$ (see Figure 6.9). In this
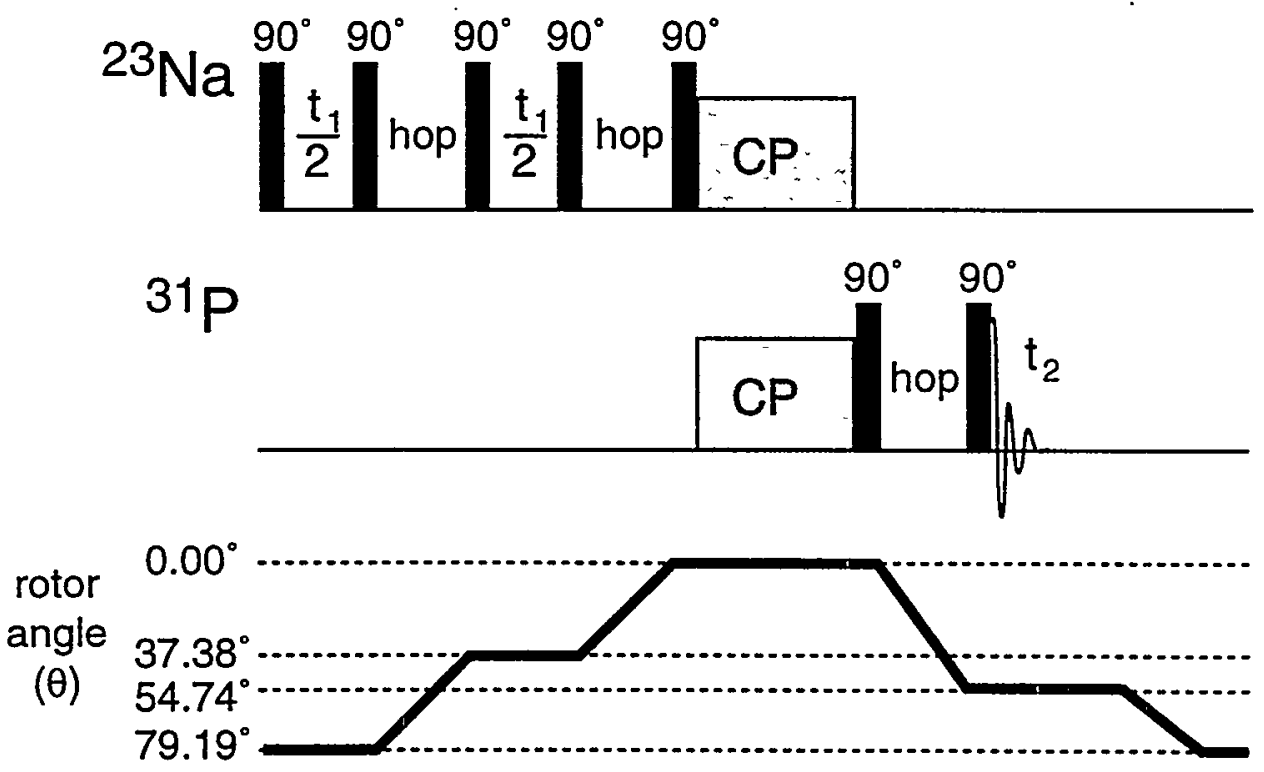

Figure 6.9 - The DAS/HETCOR experiment of Jarvie et al. ${ }^{223}$ The experiment requires three rotor axis reorientations per scan (plus one between scans). A DAS experiment removes the quadrupolar anisotropy during the evolution period. Cross polarization is then performed at a rotor angle of $0^{\circ}$ (where it is most efficient). The sample is subsequently reoriented to the magic angle for high-resolution detection of the spin-1/2 nucleus. 
experiment, the sample was first spun sequentially about the angles $79.19^{\circ}$ and $37.38^{\circ}$ with respect to the $B_{0}$ field. The evolution times at the first and second angles were chosen such that an isotropic echo was formed at the end of the $t_{1}$ evolution period (see Section 6.1.2). The magnetization was stored along $B_{0}$ using a $z$-filter while the sample was reoriented a second time to $0.00^{\circ}$. Cross polarization from the quadrupolar nucleus to the spin-1/2 nucleus was carried out at this angle (where cross polarization from quadrupolar nuclei is most efficient ${ }^{11,122}$ ). After a third reorientation (and $z$-filter on the spin-1/2 nucleus), the spin-1/2 signal was finally recorded at the magic angle. While this experiment gives high resolution in both the quadrupolar and spin-1/2 dimensions, it requires three rotor axis reorientations during each scan and, therefore, cannot be used to study nuclei with short $\mathrm{T}_{1}$ 's such as ${ }^{27} \mathrm{Al}$ or ${ }^{11} \mathrm{~B}$. It also cannot be performed on conventional equipment. The MQMAS-HETCOR experiment we developed circumvents these problems.

\subsubsection{MQMAS-HETCOR}

The pulse sequence, coherence-transfer pathway, and phase cycle for the pureabsorption mode MQMAS/HETCOR experiment is shown in Figure 6.10. As written, this sequence can be used to correlate a spin-3/2 nucleus (such as ${ }^{23} \mathrm{Na}$ ) with a spin-1/2 nucleus (such as ${ }^{31} \mathrm{P}$ ). The same principles can be applied to construct analogous pulse sequences involving higher multiple-quantum coherences if other odd-half integer quadrupolar nuclei are to be studied. The sequence depicted in Figure 6.10 shows that the MQMAS evolution period is divided into two parts as described by Equation (6.12). A single pulse is used for excitation of the triple-quantum coherence of the quadrupolar nucleus and a second pulse converts the triple-quantum coherence into single-quantum coherence. ${ }^{214,224}$ At the end of the evolution period (when the isotropic echo is formed), magnetization is transferred to the spin-1/2 nucleus by Hartmann-Hahn cross polarization, and then the ${ }^{31} \mathrm{P}$ spectrum is recorded. The result is a heteronuclear correlation experiment acquired under MAS with high-resolution in both dimensions.

The phase cycle shown in Figure 6.10 enables the collection of pure-absorption mode two-dimensional spectra by retaining a pair of "mirror-image" coherence-transfer pathways during the evolution period $t_{1} \cdot{ }^{4}$ The desired triple-quantum/single-quantum 

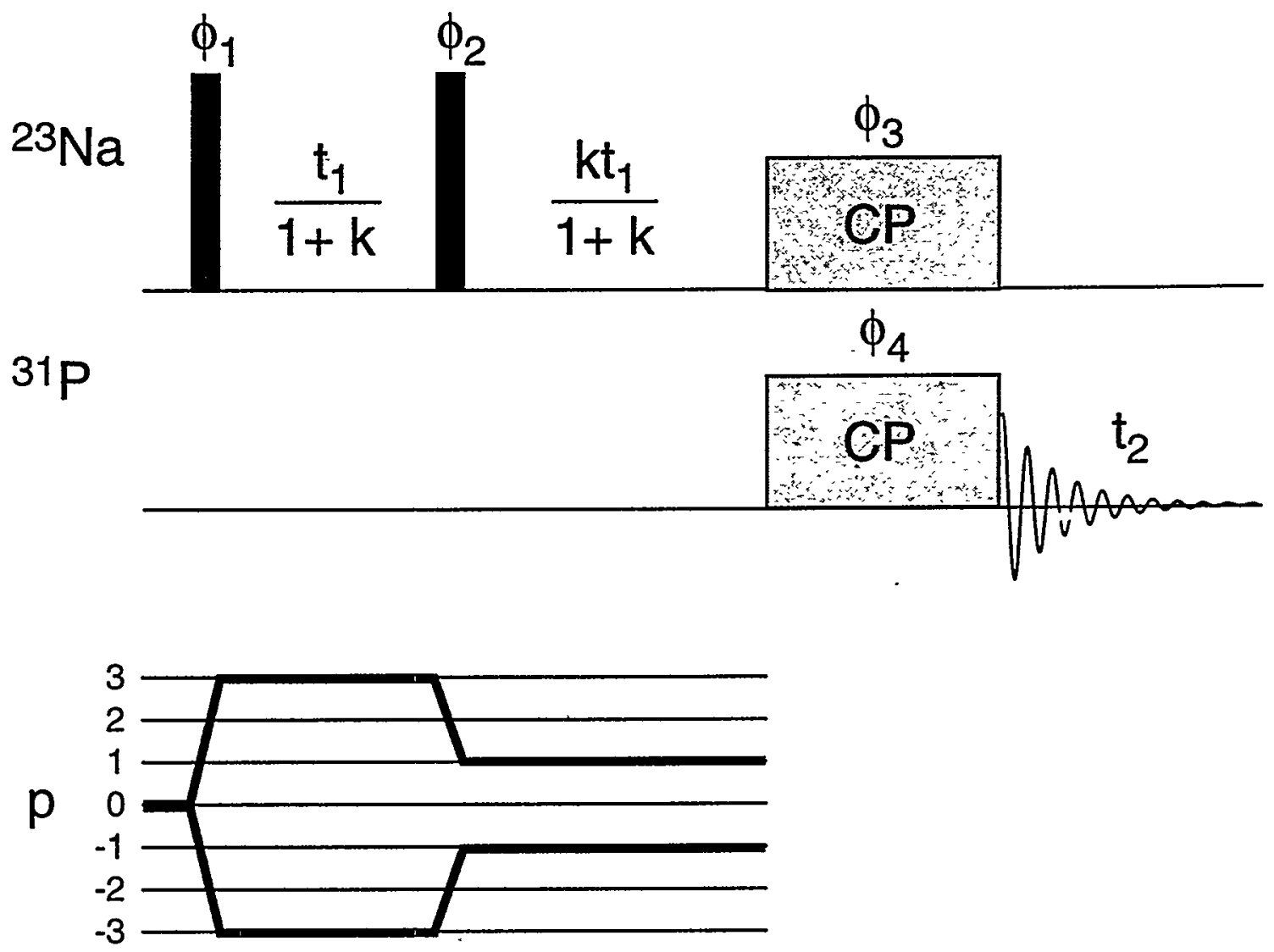

\begin{tabular}{|l|l|}
\hline$\phi_{1}$ & $0^{\circ} 60^{\circ} 120^{\circ} 180^{\circ} 240^{\circ} 300^{\circ}$ \\
\hline$\phi_{2}$ & $\left(0^{\circ}\right)_{6}\left(90^{\circ}\right)_{6}\left(180^{\circ}\right)_{6}\left(270^{\circ}\right)_{6}$ \\
\hline$\phi_{3}$ & $0^{\circ}$ \\
\hline$\phi_{4}$ & $\left(0^{\circ}\right)_{24}\left(90^{\circ}\right)_{24}\left(180^{\circ}\right)_{24}\left(270^{\circ}\right)_{24}$ \\
\hline$\phi_{R}$ & $\begin{array}{l}\left(0^{\circ} 180^{\circ}\right)_{3}\left(180^{\circ} 0^{\circ}\right)_{3}\left(90^{\circ} 270^{\circ}\right)_{3}\left(270^{\circ} 90^{\circ}\right)_{3} \\
\left(180^{\circ} 0^{\circ}\right)_{3}\left(0^{\circ} 180^{\circ}\right)_{3}\left(270^{\circ} 90^{\circ}\right)_{3}\left(90^{\circ} 270^{\circ}\right)_{3}\end{array}$ \\
\hline
\end{tabular}

Figure 6.10 - Pulse sequence, coherence-transfer pathway, and phase cycle for MQMAS/ HETCOR. "Mirror image" coherence-transfer pathways are retained during the evolution period so that two-dimensional pure absorption lineshapes can be obtained. The 96-step phase cycle incorporates CYCLOPS ${ }^{46}$ cycling and spin-temperature alternation. ${ }^{47}$ The second data set needed for States-type processing can be generated by shifting the phase of $\phi_{3}$ by $90^{\circ}$. 
pathways for the MQMAS portion of the experiment are $0 \rightarrow 3 \rightarrow 1$ and $0 \rightarrow-3 \rightarrow-1$. The phase of the first pulse is cycled through $60^{\circ}$ steps to retain $\Delta \mathrm{p}_{1}=+3$ and $\Delta \mathrm{p}_{1}=-3$; the phase of the second pulse is cycled through steps of $90^{\circ}$ to retain $\Delta \mathrm{p}_{2}=-2$ and $\Delta \mathrm{p}_{2}=+2$ (see Section 1.5). The accumulated phase at the end of $t_{1}$ is given by

$$
\sum_{\mathrm{i}}\left(\Delta \mathrm{p}_{\mathrm{i}}\right) \varphi_{\mathrm{i}}=\Delta \mathrm{p}_{1} \phi_{1}+\Delta \mathrm{p}_{2} \phi_{2}
$$

For the phase cycle and the two desired paths of Figure 6.10 , this corresponds to

$$
\left\{\left(0^{\circ}, 180^{\circ}, 0^{\circ}, 180^{\circ}, 0^{\circ}, 180^{\circ}, 180^{\circ}, 0^{\circ}, 180^{\circ}, 0^{\circ}, 180^{\circ}, 0^{\circ}\right){ }_{2}\right\}
$$

When this is combined with a constant-phase $\mathrm{CP}$ pulse, spin-temperature alternation is automatically achieved. ${ }^{47}$

Note that any residual triple-quantum coherences that were not converted to single-quantum coherences by the second pulse could potentially also be transferred in a cross-polarization step since the effective nutation frequency of the triple-quantum coherence (Equation (6.8)) would match the nutation frequency of the spin-1/2 nucleus at some point during the rotor cycle. However, such coherences (corresponding to the paths $0 \rightarrow 3 \rightarrow 3$ and $0 \rightarrow-3 \rightarrow-3$ ), would accumulate phases

$$
\left\{\left(0^{\circ}, 180^{\circ}, 0^{\circ}, 180^{\circ}, 0^{\circ}, 180^{\circ}, 0^{\circ}, 180^{\circ}, 0^{\circ}, 180^{\circ}, 0^{\circ}, 180^{\circ}\right)_{2}\right\}
$$

Proper cycling of the receiver in accordance with Equation (6.16) will cancel out such triple-quantum signals.

To obtain a pure-phase, two-dimensional spectrum with frequency discrimination in the $\omega_{1}$ dimension, two amplitude-modulated data sets must be collected and processed according to the method of States et al. ${ }^{51}$ In our experiment, this second data set is generated by using a phase cycle identical to that shown in Figure 6.10, except with $\phi_{3}=90^{\circ}$. Finally, CYCLOPS ${ }^{46}$ phase cycling of $\phi_{4}$ is included to eliminate receiver imbalance. The complete phase cycle has 96 steps. 
To test the MQMAS/HETCOR pulse sequence, experiments were performed on a sample of anhydrous sodium trimetaphosphate $\left(\mathrm{Na}_{3} \mathrm{P}_{3} \mathrm{O}_{9}\right)$ which was prepared according the method of Jarvie et al. ${ }^{223}$ This is the same sample that was used to demonstrate the feasibility of the DAS/HETCOR experiment, ${ }^{223}$ and its crystal structure (as determined by $\mathrm{X}$-ray studies ${ }^{225}$ ) is depicted in Figure 6.11. Sodium trimetaphosphate is known to have two crystallographically distinct sodium sites and two crystallographically distinct phosphorous sites; in both cases, the "general" site has twice the population of the "mirror" site. Table 6.2 indicates the nearest Na-P distances.

Figure 6.12 shows the ${ }^{23} \mathrm{Na}$ MQMAS spectrum of sodium trimetaphosphate recorded at $11.7 \mathrm{~T}$ using shifted-echo, hypercomplex processing and shearing. ${ }^{53}$

The MQMAS/HETCOR spectrum of sodium trimetaphosphate is shown in Figure 6.13. The spectrum was recorded on a Chemagnetics CMX-500 spectrometer using a 7.5 $\mathrm{mm}$ Chemagnetics probe that was double-tuned to $131.894 \mathrm{MHz}$ for ${ }^{23} \mathrm{Na}$ and 201.850 $\mathrm{MHz}$ for ${ }^{31} \mathrm{P}$. Each of the first two pulses was $16 \mu \mathrm{s}$, corresponding to a $3 \pi$ rotation on the central transition of sodium. This served to partially suppress the direct excitation of the

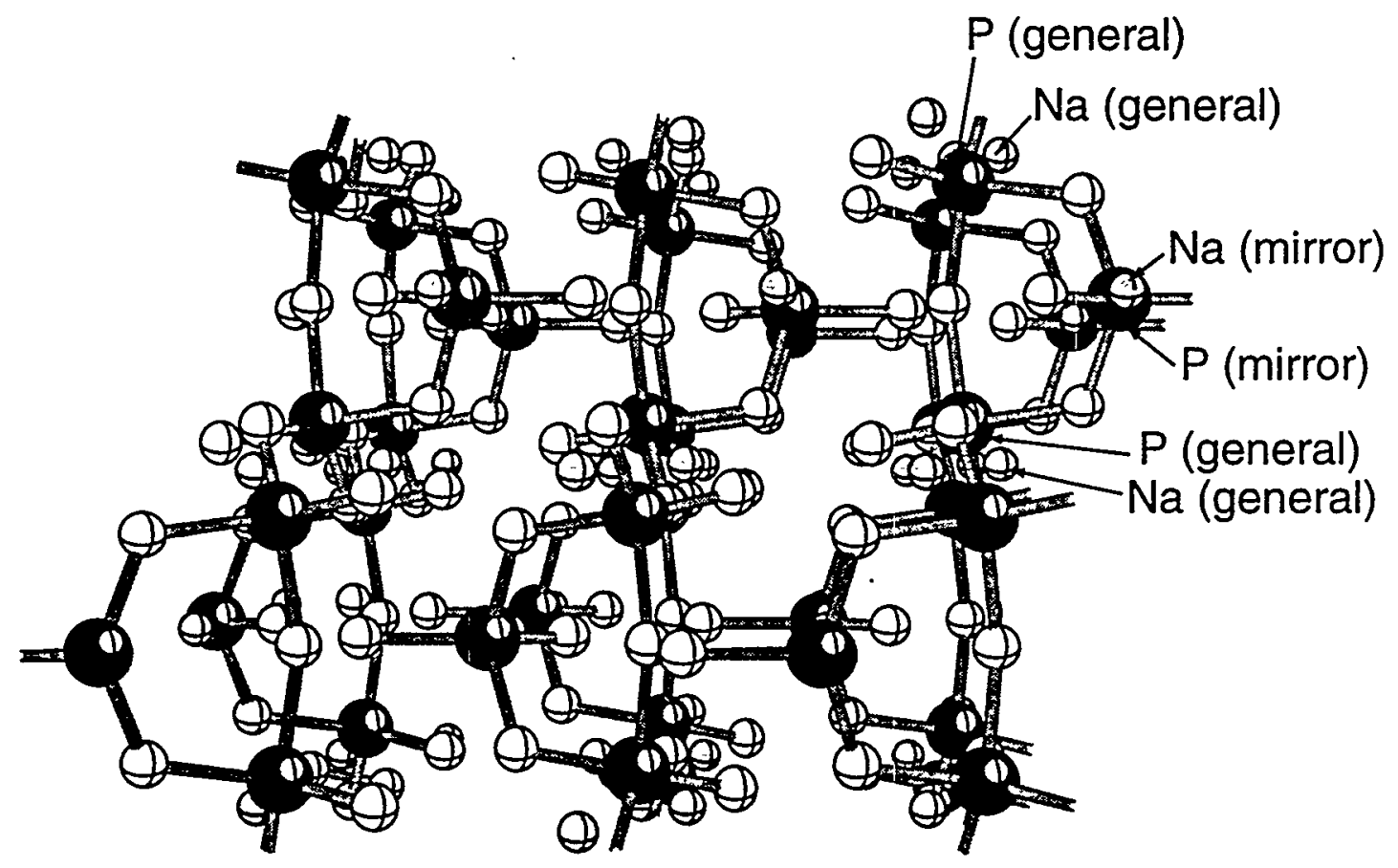

Figure 6.11 - The crystal structure of $\mathrm{Na}_{3} \mathrm{P}_{3} \mathrm{O}_{9}$ as determined by X-ray crystallography. ${ }^{225}$ The crystal has orthorhombic symmetry. The two types of sodium and phosphorous sites are indicated. Note that there are twice as many general sites as mirror sites. The nearest Na-P distances are listed in Table 6.2. 


\begin{tabular}{|c|c|c|c|}
\hline $\begin{array}{c}\text { Na(mirror) to } \\
\text { P(mirror) }\end{array}$ & $\begin{array}{c}\text { Na(mirror) to } \\
\text { P(general) }\end{array}$ & $\begin{array}{c}\text { Na(general) to } \\
\text { P(mirror) }\end{array}$ & $\begin{array}{c}\text { Na(general) to } \\
\text { P(general) }\end{array}$ \\
\hline \hline $3.466 \AA$ & $3.301 \AA$ & $3.299 \AA$ & $3.323 \AA$ \\
$4.686 \AA$ & $3.434 \AA$ & $3.380 \AA$ & $3.452 \AA$ \\
{$[5.728 \AA]$} & $5.675 \AA$ & $5.458 \AA$ & $3.584 \AA$ \\
& $5.816 \AA$ & $5.622 \AA$ & $4.614 \AA$ \\
& {$[5.892 \AA]$} & {$[5.987 \AA]$} & $4.770 \AA$ \\
& & & $4.810 \AA$ \\
& & & $4.905 \AA$ \\
& & & $5.530 \AA$ \\
& & & {$[5.702 \AA]$} \\
& & & {$[5.765 \AA]$} \\
\hline
\end{tabular}

Table 6.2 - Nearest distances between sodium and phosphorous sites in anhydrous sodium trimetaphosphate as calculated from the X-ray structure. ${ }^{225}$ All distances of under $6 \AA$ are listed. The unbracketed distances were used to estimate theoretical peak intensities.

single-quantum coherence. ${ }^{53}$ The cross-polarization contact time was $10 \mathrm{~ms}$ and the spinning speed was $5 \mathrm{kHz}$. The $\mathrm{n}=-1$ match-condition sideband (see Section 4.3) was used for cross polarization and corresponded to a spin-lock strength of $4.6 \mathrm{kHz}$. This gave adiabaticity parameters of $\alpha<0.005$ for the spin lock on both ${ }^{23} \mathrm{Na}$ sites, which is well within the sudden regime. Thirty-five complex $t_{1}$ points consisting of 960 scans in each were collected with a recycle delay of $3 \mathrm{~s}$. The projections in each dimension show that two peaks are observed for each nucleus, corresponding to the crystallographically distinct

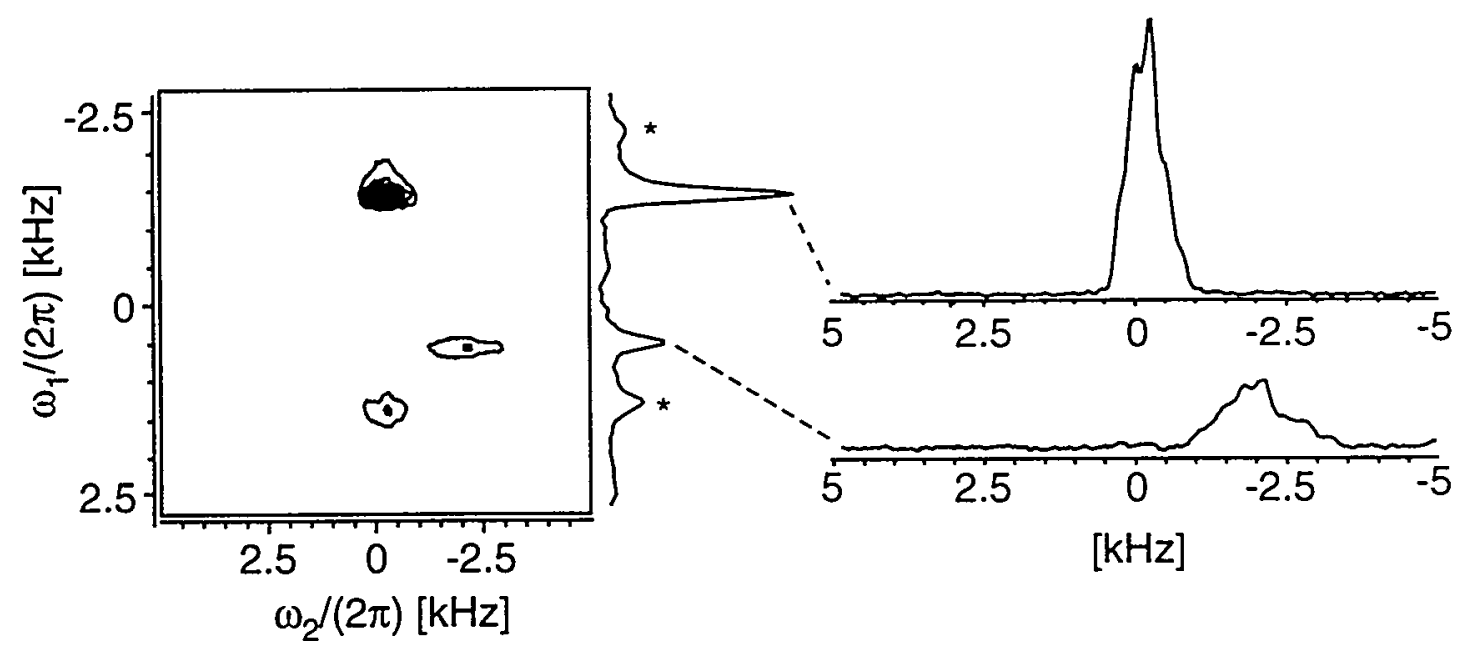

Figure 6.12 - Sheared ${ }^{23} \mathrm{Na}$ MQMAS spectrum of $\mathrm{Na}_{3} \mathrm{P}_{3} \mathrm{O}_{9}$. Thirty-one $\mathrm{t}_{1}$ slices with 24 scans in each were acquired with a $3 \mathrm{~s}$ recycle delay. 
sites. $^{225}$ The two phosphorous peaks are at -18.7 and $-15.5 \mathrm{ppm}$ with respect to $85 \%$ $\mathrm{H}_{3} \mathrm{P}_{3} \mathrm{O}_{4}$ at $0 \mathrm{ppm}$, and the two peaks in the ${ }^{23} \mathrm{Na}$ spectrum are at -6.1 and $-21.5 \mathrm{ppm}$ with respect to solid $\mathrm{NaCl}$ at $0 \mathrm{ppm}$.

Table 6.3 lists the quadrupolar parameters and isotropic chemical shifts (referenced to solid $\mathrm{NaCl}$ ) for the two sodium sites in $\mathrm{Na}_{3} \mathrm{P}_{3} \mathrm{O}_{9}$ as previously determined by Koller et al. ${ }^{226}$ from fits of a one-dimensional MAS spectrum recorded at $9.4 \mathrm{~T}$. The values we obtained from fits of anisotropic slices of an MQMAS spectrum recorded at 4.2 $\mathrm{T}$ are also listed. By using these parameters and Equations (6.7) and (6.14), it was possible to predict the positions of the peaks in DAS and MQMAS experiments performed at various field strengths. These predictions are listed in Table 6.3 and are compared with experimental results when they are available. Note that the measured values reported in Jarvie's paper ${ }^{223}$ were referenced to $0.1 \mathrm{M} \mathrm{NaCl}$, which has a chemical shift of $\delta=-7.2 \mathrm{ppm}$ relative to solid $\mathrm{NaCl}^{226}$ To make the comparison easier, all shifts are listed in the table relative to solid $\mathrm{NaCl}$.

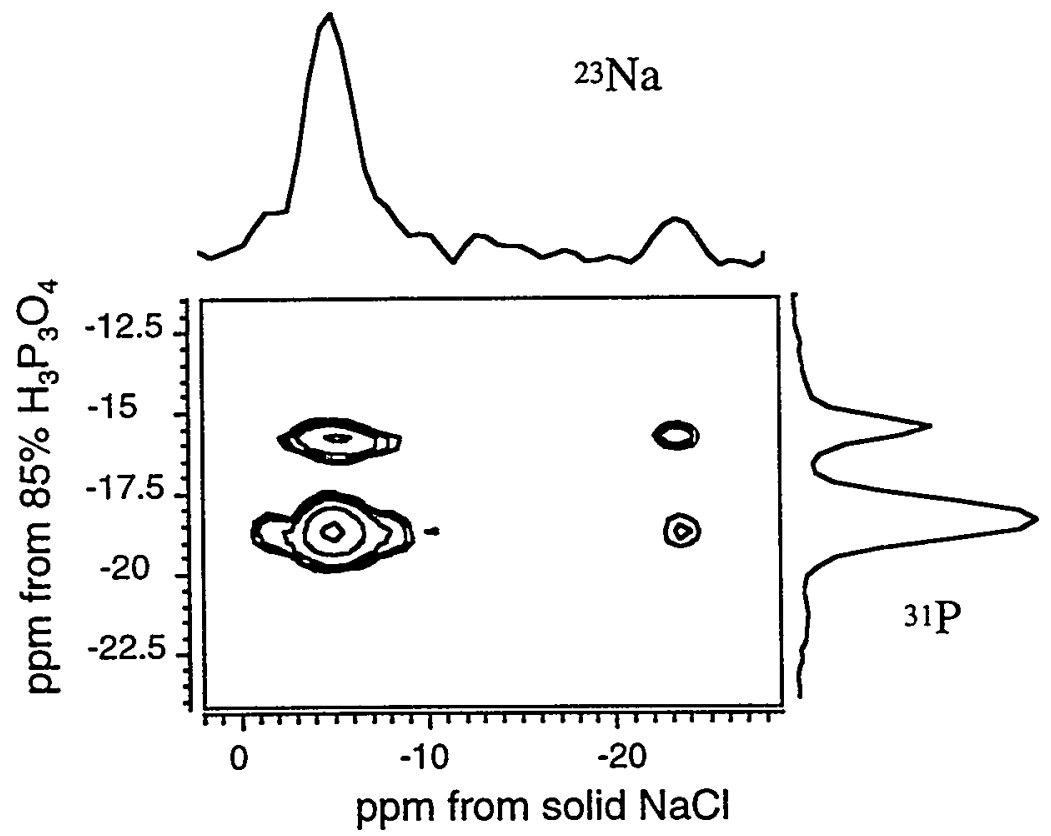

Figure $6.13-{ }^{23} \mathrm{Na} /{ }^{31} \mathrm{P}$ MQMAS/HETCOR spectrum of $\mathrm{Na}_{3} \mathrm{P}_{3} \mathrm{O}_{9}$ recorded with a cross polarization contact time of $10 \mathrm{~ms}$ and a spinning speed of $5 \mathrm{kHz}$. Thirty-five complex $\mathrm{t}_{1}$ points consisting of 960 scans in each were collected with a recycle delay of $3 \mathrm{~s}$. 


\begin{tabular}{|c|c|c|c|}
\hline Parameter & Source & $\begin{array}{l}\text { General } \\
\text { Site }\end{array}$ & Mirror Site \\
\hline $\mathrm{C}_{\mathrm{qcc}}$ & Koller et al. & $1.57 \mathrm{MHz}$ & $2.20 \mathrm{MHz}$ \\
\hline$\eta$ & Koller et al. & 0.55 & 0.70 \\
\hline$\delta_{i s o}^{(C S)}$ & Koller et al. & $-5.60 \mathrm{ppm}$ & $-14.80 \mathrm{ppm}$ \\
\hline $\mathrm{C}_{\mathrm{qcc}}$ & our measurements & $1.57 \mathrm{MHz}$ & $2.20 \mathrm{MHz}$ \\
\hline$\eta$ & our measurements & 0.55 & 0.64 \\
\hline$\delta_{\text {iso }}^{(C S)}$ & our measurements & $-5.20 \mathrm{ppm}$ & $-15.07 \mathrm{ppm}$ \\
\hline$\delta_{\text {iso }}^{\text {DAS }}(9.4 \mathrm{~T} ;$ calculated $)$ & $\begin{array}{l}\text { calculation based on the } \\
\text { quadrupolar parameters of } \\
\text { Koller et al. }\end{array}$ & $-11.7 \mathrm{ppm}$ & $-27.4 \mathrm{ppm}$ \\
\hline$\delta_{\text {iso }}^{\text {DAS }}(9.4 \mathrm{~T} ;$ calculated $)$ & $\begin{array}{l}\text { calculation based on the } \\
\text { quadrupolar parameters } \\
\text { we determined }\end{array}$ & $-11.3 \mathrm{ppm}$ & $-27.4 \mathrm{ppm}$ \\
\hline$\delta_{\text {iso }}^{\text {DAS }}(9.4 \mathrm{~T} ;$ measured $)$ & measured by Jarvie et al. & $-9.7 \mathrm{ppm}$ & $-26.1 \mathrm{ppm}$ \\
\hline$\delta_{\text {iso }}^{\text {DAS }}(11.7 \mathrm{~T} ;$ calculated $)$ & $\begin{array}{l}\text { calculation based on the } \\
\text { quadrupolar parameters of } \\
\text { Koller et al. }\end{array}$ & $-9.5 \mathrm{ppm}$ & $-22.9 \mathrm{ppm}$ \\
\hline$\delta_{\text {iso }}^{\text {DAS }}(11.7 \mathrm{~T} ;$ calculated $)$ & $\begin{array}{l}\text { calculation based on the } \\
\text { quadrupolar parameters } \\
\text { we determined }\end{array}$ & $-9.1 \mathrm{ppm}$ & $-23.0 \mathrm{ppm}$ \\
\hline$\delta_{\text {iso }}^{\text {MQMAS }}(11.7 \mathrm{~T} ;$ calculated $)$ & $\begin{array}{l}\text { calculation based on the } \\
\text { quadrupolar parameters of } \\
\text { Koller et al. }\end{array}$ & $-7.0 \mathrm{ppm}$ & $-21.3 \mathrm{ppm}$ \\
\hline$\delta_{\text {iso }}^{\text {MQMAS }}(11.7 \mathrm{~T}$; calculated $)$ & $\begin{array}{l}\text { calculation based on the } \\
\text { quadrupolar parameters } \\
\text { we determined }\end{array}$ & $-6.2 \mathrm{ppm}$ & $-22.1 \mathrm{ppm}$ \\
\hline$\delta_{\text {iso }}^{\mathrm{MQMAS}}(11.7 \mathrm{~T}$; measured $)$ & our measurements & $-6.1 \mathrm{ppm}$ & $-21.5 \mathrm{ppm}$ \\
\hline
\end{tabular}

Table 6.3 - Quadrupolar parameters, predicted shifts, and measured shifts for the ${ }^{23} \mathrm{Na}$ sites in $\mathrm{Na}_{3} \mathrm{P}_{3} \mathrm{O}_{9}$. Shifts calculated both from the parameters of Koller et al. ${ }^{226}$ and from our parameters are listed and are compared to the DAS/HETCOR experiment of Jarvie et al. ${ }^{223}$ and to our MQMAS/HETCOR experiment. Predicted shifts for a DAS experiment at $11.7 \mathrm{~T}$ are also tabulated. All shifts are reported relative to solid $\mathrm{NaCl}$ at $0 \mathrm{ppm}$. 
The positions of the peaks we measured in our MQMAS/HETCOR experiment agree, within experimental error, with the theoretically predicted shifts. (The DAS shifts of Jarvie et al. appear to deviate more from the predicted shifts, but the peaks are quite broad and the resolution relatively low in the $\omega_{1}$ dimension. These factors could explain at least some of the discrepancy.)

The 2-D MQMAS/HETCOR spectrum (Figure 6.13) shows four distinct cross peaks between the two ${ }^{31} \mathrm{P}$ and two ${ }^{23} \mathrm{Na}$ resonances. Correlation between the two nuclei is principally through dipolar coupling, which results from both sodium sites being in close proximity to both phosphorous sites. As in the DAS version of the experiment, the measured intensities of the cross peaks in this new experiment may not yet be considered quantitative. Cross polarization is often not quantitative even when quadrupolar nuclei are absent, but in an idealized HETCOR experiment between spin-1/2 nuclei (using the pulse sequence of Figure 6.7), peak intensities would be influenced by three factors: (1) the population statistics (how many nuclei of each type are present), (2) the distances between heteronuclei (which determine the rate of cross polarization), and (3) the relative relaxation times $\left(T_{1 \rho}\right)$ of spins. in different sites. If the $T_{1 \rho}$ 's for a given isotope are similar, relative crosss-peak intensities will be proportional to the rates of cross relaxation which in turn are proportional to heteronuclear second moments. ${ }^{227}$ We can estimate "ideal" relative intensities for the cross peaks in our system by using the distances from Table 6.2 to calculate second moments. In this approximation, we assume that we can average the angular-dependent terms over all powder orientations ${ }^{8}$ so all that remains is to calculate the sum $\sum_{\mathrm{i}<\mathrm{j}} 1 / \mathrm{r}_{\mathrm{ij}}^{6}$ over the nearest Na-P distances (the number of terms in the sum being determined by the population statistics). The "ideal" relative intensities would then be 1:0.7:0.7:0.3 for the $\mathrm{Na}$ (general)/P(general), $\mathrm{Na}$ (general)/P(mirror), $\mathrm{Na}$ (mirror)/ $\mathrm{P}$ (general), and $\mathrm{Na}$ (mirror)/P(mirror) cross peaks, respectively.

In the MQMAS/HETCOR experiment, even further factors affect the cross-peak intensities. First, as discussed in Chapters 3 and 4, cross-polarization dynamics of quadrupolar nuclei are complicated under magic-angle spinning conditions by the time dependence of the first-order quadrupolar interaction. This is not the case for the DAS/ HETCOR experiment since the polarization transfer occurs at $0^{\circ}$ to $\mathrm{B}_{0}$, permitting attainment of the full, static cross-polarized intensity. ${ }^{11}$ The second factor that complicates 
the quantification of the MQMAS/HETCOR experiment is that both the excitation of the triple-quantum coherence and its conversion back to single-quantum coherence depend strongly upon the if excitation power and the quadrupolar parameters. ${ }^{212}$ For the excitation and conversion pulses that we used (see Figure 6.12), the site with the greater $\mathrm{C}_{\mathrm{qcc}}$ had one-quarter the intensity of the other site, despite the fact that its population is one half that of the other site. The measured relative cross-peak intensities for the spectrum in Figure 6.13 are 1:0.5:0.2:0.1 for $\mathrm{Na}$ (general)/P(general), $\mathrm{Na}$ (general)/ $\mathrm{P}$ (mirror), $\mathrm{Na}$ (mirror)/P(general), and $\mathrm{Na}$ (mirror)/P(mirror), respectively, which deviates from the ideal case due to these complications. Modifications of the original MQMAS experiment to make the intensities closer to quantitative have been proposed by Wu et al., 228 and this is currently an active area of research in many laboratories. However, it is important to note that the qualitative appearance of our MQMAS/HETCOR spectrum is similar to the DAS version; the DAS experiment is also not strictly quantitative. ${ }^{223}$

The advantage of combining the MQMAS experiment with the HETCOR experiment is immediately obvious when Figure 6.13 is compared with a normal MAS HETCOR spectrum of $\mathrm{Na}_{3} \mathrm{P}_{3} \mathrm{O}_{9}$ (Figure 6.14). Even though the second-order quadrupolar interaction has not been completely averaged, the ${ }^{23} \mathrm{Na}$ dimension for this sample still exhibits relatively high resolution because of the significant differences in $C_{q c c}, \eta$, and $\delta_{\text {iso }}^{(\mathrm{CS})}$ between the two sites. However, even for this ideal case it is clear that the MQMAS/HETCOR experiment gives superior resolution. For more complex systems where the ${ }^{23} \mathrm{Na}$ dimension is not so well resolved, such as sodium phosphate glasses, the MQMAS/HETCOR experiment should be of significant utility.

In principle, the heteronuclear correlation experiment could be applied in reverse, transferring the magnetization from ${ }^{31} \mathrm{P}$ to ${ }^{23} \mathrm{Na}$ and then performing the $3 \mathrm{Q} / 1 \mathrm{Q} \mathrm{MQMAS}$ experiment to obtain high resolution in the ${ }^{23} \mathrm{Na}$ dimension (possibly with direct cross polarization of the triple-quantum transition which would occur nine times faster than single-quantum cross polarization for a given spin-lock field strength on the ${ }^{31} \mathrm{P}$ channel ${ }^{136,138}$ ). However, performing the experiment in this way has two major drawbacks. The first is that the experiment would, in effect, become a three-dimensional experiment, increasing the time required to collect the data. The second disadvantage is that the $T_{1}$ of sodium is typically much shorter than that of phosphorous due to the 
efficiency of quadrupolar relaxation mechanisms. For example, in $\mathrm{Na}_{3} \mathrm{P}_{3} \mathrm{O}_{9}$ the delay between scans necessary to prevent significant saturation of the signal is $3 \mathrm{~s}$ for ${ }^{23} \mathrm{Na}$ and $660 \mathrm{~s}$ for ${ }^{31} \mathrm{P}$; performing cross polarization from ${ }^{31} \mathrm{P}$ to ${ }^{23} \mathrm{Na}$ would increase the experimental time prohibitively.

Although cross polarization is less efficient for samples spun at the magic angle than for samples spun at an angle of $0^{\circ}$ with respect to the static field, ${ }^{122}$ the MQMAS/ HETCOR experiment has several advantages compared to the DAS version. The main advantage is that high-resolution HETCOR spectra may be obtained from quadrupolar nuclei using a conventional MAS NMR probe. Our experiments were performed using an unmodified Chemagnetics probe with a $7.5 \mathrm{~mm}$ rotor and an rf field strength of only 42 $\mathrm{kHz}$ for the multiple-quantum coherence excitation. As double-resonance MAS probes are available in most solid-state NMR laboratories, this simplification will enable this

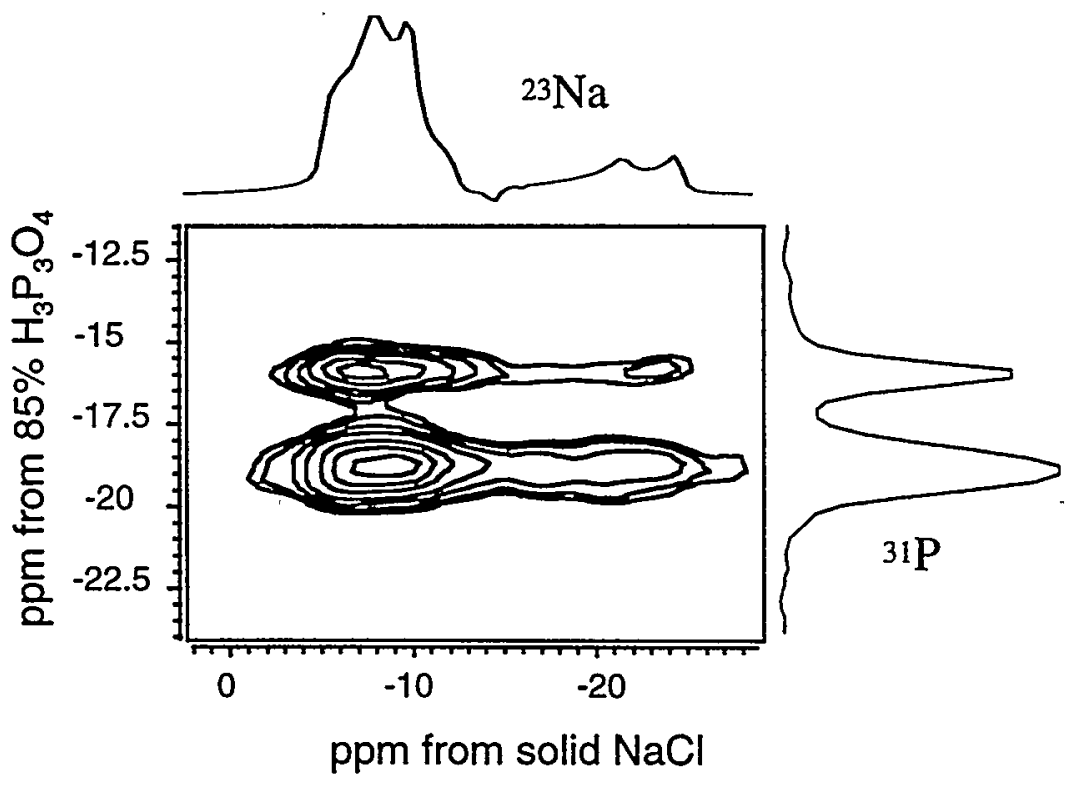

Figure 6.14 - A conventional two-dimensional ${ }^{23} \mathrm{Na} /{ }^{31} \mathrm{P}$ MAS HETCOR spectrum of $\mathrm{Na}_{3} \mathrm{P}_{3} \mathrm{O}_{9}$ recorded under similar conditions to those in Figure 6.13 . The $90^{\circ}$ pulse length of ${ }^{23} \mathrm{Na}$ was $27 \mu \mathrm{s}$, the cross polarization contact time was $10 \mathrm{~ms}$, and the spinning speed was $5 \mathrm{kHz}$. Thirty-two complex $t_{1}$ points consisting of 256 scans were collected with a recycle delay of $3 \mathrm{~s}$. 
experiment to be widely applied. In contrast, the DAS/HETCOR experiment ${ }^{223}$ requires a static-coil dynamic-angle spinning probe that is capable of cross polarization at $0^{\circ}$ with respect to $\mathrm{B}_{0} ;{ }^{155.156}$ such probes are not currently commercially available.

A second advantage of the MQMAS/HETCOR experiment is that, potentially, the resolution of the ${ }^{23} \mathrm{Na}$ dimension will be greater than that observed in the DAS/HETCOR experiment, hence increasing the possibility of separating signals from sites with similar chemical environments. ${ }^{216}$ This arises from scaling of the chemical and quadrupolar shifts in the 3Q/1Q MQMAS experiment on an $S=3 / 2$ nucleus by $17 / 8$ and $-5 / 4$, respectively (see Equation (6.14) and Table 6.1). In Table 6.3, peak positions for both a DAS and an MQMAS experiment at $11.7 \mathrm{~T}$ were calculated for the sodium sites in $\mathrm{Na}_{3} \mathrm{P}_{3} \mathrm{O}_{9}$. Although the numerical values differ slightly depending on which set of quadrupolar parameters are used, the dispersion in the MQMAS spectrum is greater than that in the DAS spectrum in both cases.

A third advantage of the MQMAS/HETCOR experiment, and the most important for its application to a wide variety of materials, is that samples with short $T_{1}$ 's may be investigated. DAS/HETCOR is limited to samples where $T_{1}$ is greater than about $150 \mathrm{~ms}$ since the typical time required to flip the spinning axis is about $40 \mathrm{~ms}$, and the experiment requires three sample reorientations (see Figure 6.9). This has previously excluded the study of many ${ }^{27} \mathrm{Al}$ and ${ }^{11} \mathrm{~B}$ systems. 


\section{Chapter 7: Reversal of Radiofrequency-Driven Spin Diffusion by Reorientation of the Sample- Spinning Axis}

The previous three chapters have examined heteronuclear polarization transfer in the form of cross polarization. In this chapter, we viill be concerned with homonuclear polarization transfer, or "spin diffusion," in systems of spin-1/2 nuclei. In particular, we will demonstrate that it is possible to reverse the process of rf-driven spin diffusion by changing the orientation of the sample-spinning axis relative to the static magnetic field.

\subsection{Previous Polarization-Echo Experiments}

The ability to manipulate the nuclear spin Hamiltonian during the course of an NMR experiment has permitted the observation of a variety of echo effects. The wellknown echo experiments by $\mathrm{Hahn}^{229}$ demonstrated that inhomogeneous interactions could be refocused by two radiofrequency pulses. Since then, spin echoes have also been observed in homogeneously broadened systems. The "magic-echo experiment" introduced by Schneider et al. ${ }^{230}$ and Rhim et al. ${ }^{231}$ showed that it was possible to induce a "time reversal" of the free-induction decay (FID) in a dipolar-coupled spin system. Llor et al. ${ }^{232}$ reported the observation of the time reversal of isotropic many-body spin couplings in zero-field NMR.

More recently, several experiments have demonstrated the possibility of refocusing the process of homonuclear polarization transfer, or "spin diffusion," 233 in extended spin systems. Exploiting the fact that the truncated dipolar Hamiltonians in the rotating and laboratory frames have opposite signs, ${ }^{5}$ Zhang et al. ${ }^{234}$ designed a pulse sequence to refocus proton spin diffusion in a static sample. Karlsson et al. ${ }^{235}$ and Tomaselli et al..$^{236}$ showed that the polarization-transfer process could also be refocused under MAS $^{237,238}$ conditions by using rotational-resonance recoupling 239,240 or rotorsynchronized multiple-pulse sequences. The formation of such polarization echoes clearly demonstrates the deterministic quantum-mechanical nature of the "spin-diffusion" process even though it can in some cases be approximated by a diffusion equation..$^{8,233,241,242}$ 
The dependence of the NMR Hamiltonian on both spatial and spin variables ${ }^{7,8}$ suggests that the process of spin diffusion might also be reversible by a mechanical sample reorientation. It has previously been shown that a "magic echo" of the freeinduction decay can be induced in an oriented liquid-crystalline sample by changing the angle between the director and the external magnetic field. ${ }^{243}$ In this chapter, it will be shown that a time reversal of spin diffusion can be achieved by switching the axis of sample rotation during a radio-frequency-driven spin-diffusion experiment. ${ }^{244}$

\subsection{Spin Diffusion}

The term "spin diffusion" is commonly used to refer to the transfer of polarization among like nuclei. ${ }^{23,242}$ This process is meditated through the zero-quantum "flip-flop" term in the dipolar Hamiltonian (the second term on the right in Equation (1.72)) and is very efficient when the nuclei have the same resonance frequency. When the nuclei have different frequencies, however, the "flip-flop" is not energy-conserving, and polarization transfer will not occur unless the system can obtain compensating energy from another source.

Among protons, spin diffusion occurs readily since chemical-shift differences are small relative to dipolar couplings. However, the opposite is true for rare spins such as natural abundance ${ }^{13} \mathrm{C}$. The rate constant for spin diffusion, $\mathrm{W}_{\mathrm{jk}}$, between two spins $\mathrm{S}_{\mathrm{j}}$ and $S_{k}$ in a static sample can be estimated using Fermi's Golden Rule ${ }^{1,8}$

$$
W_{j k}=\frac{\pi}{2} s^{2} b_{e f f, j k}^{2} F_{j k}(0)
$$

where $s$ is a pulse-sequence-dependent scaling factor, and $b_{e f f, j k}$ is the effective dipolar coupling frequency (explicit examples of which will be given below). The term $F_{j k}(0)$ is the intensity of the normalized zero-quantum spectrum of the two spins at frequency zero; ${ }^{8,245,246}$ it represents the fraction of transitions which will be energy-conserving. The zero-quantum line is centered at the difference frequency $\left(\Omega_{j}-\Omega_{\mathrm{k}}\right)$ of the two spins (see Figure 7.1) which means that the closer their frequencies, the greater the value of $F_{j k}(0)$. 
For natural-abundance ${ }^{13} \mathrm{C}$ spins, chemical-shift differences will often be much greater than the dipolar-coupling frequency, which would seem to imply that $F_{j k}(0)$, and, hence, the rate of spin diffusion, would be zero. However, coupling to an abundant proton bath can broaden the zero-quantum line so that spin diffusion is possible. This mechanism is commonly known as "proton-driven" spin diffusion although it is important to realize that the magnetization is not transferred to the protons during this process..$^{23}$

Proton-driven spin diffusion can be measured using a two-dimensional pulse sequence that is identical to that used to measure chemical exchange (see Figure 2.7). After cross polarization and a frequency-labeling period $\left(t_{1}\right)$, spin diffusion is permitted to occur during a mixing time (in the range of hundreds of milliseconds to hundreds of seconds), and then the signal is recorded. Such experiments have been used to determine relative tensor orientations ${ }^{248,249}$ and to probe proximities in heterogeneous materials ${ }^{23}$ The rate of proton-driven spin diffusion tends to be very slow because $F_{j k}(0)$, though not zero, is still small (see Figure 7.1a). However, the rate of spin diffusion among rare spins can be significantly enhanced by coupling the system to mechanical rotation of the sample

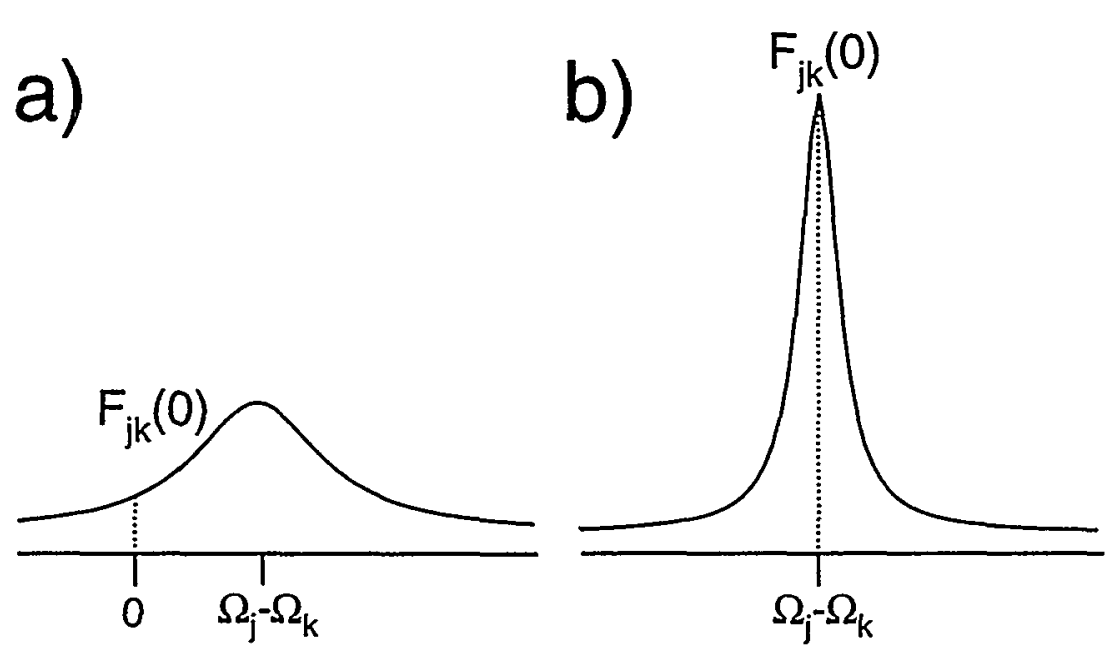

Figure 7.1 - Schematic of zero-quantum lineshapes for (a) proton-driven and (b) rf-driven spin diffusion between a pair of spins. The zero-quantum lineshape is assumed to be Lorentzian ${ }^{247}$ and is centered at the difference frequency of the two spins. The rate of spin diffusion is proportional to the intensity of the zero quantum lineshape at zero frequency. The rf-driving process both narrows the width of the zero-quantum line and moves its center closer to zero frequency, dramatically enhancing the rate of spin diffusion. 
during MAS ("rotor-driven" spin diffusion) $)^{239,240}$ or by using if fields to reduce chemicalshift differences ("rf-driven" spin diffusion). ${ }^{244,250}$ The faster rates permit studies of shortrange order.

In rf-driven spin diffusion, a spin-lock field of strength $\omega_{1 \mathrm{~S}}$ is applied to the dilute $\mathrm{S}$ spins $\left({ }^{13} \mathrm{C},{ }^{15} \mathrm{~N}\right)$ during the mixing time. ${ }^{24,250}$ This spin-lock field scales the chemicalshift differences among the $S$ spins virtually to zero and decouples them from the abundant I spins $\left({ }^{1} \mathrm{H}\right)$. These effects enhance the rate of spin diffusion among the dilute spins by several orders of magnitude by moving the center of the zero-quantum line closer to $\omega=0$ and by narrowing its linewidth (see Figure 7.1b). ${ }^{244,245}$ For a spin-lock field which is applied along the $\mathrm{x}$-axis in the rotating frame and exceeds the dipolar interactions in its strength, the average Hamiltonian which drives the spin-diffusion process in a static sample is given to zeroth order by

$$
\overline{\mathcal{H}}_{\text {static }}^{(0)}=s \sum_{j<k} \hbar b_{j k}\left(r_{j k}, \vartheta_{j k}\right)\left[3 S_{j x} S_{k x}-\left(S_{j} \cdot S_{k}\right)\right]
$$

where $s$ is a scaling factor that equals $-\frac{1}{2}$ for an on-resonance, continuous-wave (cw) spin lock ${ }^{5}$ and $b_{j k}$ is an effective dipolar coupling frequency

$$
b_{j k}\left(r_{j k}, \vartheta_{j k}\right)=-\left(\frac{\mu_{0}}{4 \pi}\right)\left(\frac{\gamma_{s}^{2} \hbar}{r_{j k}^{3}}\right) \frac{1}{2}\left(3 \cos ^{2} \vartheta_{j k}-1\right)=-d_{j k} P_{2}\left(\cos \vartheta_{j k}\right)
$$

The angle of the internuclear vector of the spins $\mathrm{j}$ and $\mathrm{k}$ with respect to the external magnetic field is given by $\vartheta_{\mathrm{jk}}$, and $\mathrm{d}_{\mathrm{jk}}$ is the dipolar coupling constant.

The truncated Hamiltonian of Equation (7.2) is rendered time-dependent by sample rotation about an axis inclined at an angle $\theta$ from the direction of $\mathrm{B}_{0}{ }^{7.251}$ Under the condition $\left|s b_{j k}\right| \ll \omega_{r} \ll \omega_{1 S}$, zeroth-order average-Hamiltonian theory can again be applied to the Hamiltonian already truncated by the if field. This approximation corresponds to neglecting all of the time-dependent terms, and the secular Hamiltonian for the spin-diffusion process in the rotating sample becomes

$$
\overline{\mathcal{H}}_{\mathrm{rot}}^{(0)}=\mathrm{P}_{2}(\cos \theta) \mathrm{s} \sum_{\mathrm{j}<\mathrm{k}} \hbar \mathrm{b}_{\mathrm{jk}}\left(\mathrm{r}_{\mathrm{jk}}, \beta_{\mathrm{jk}}\right)\left[3 \mathrm{~S}_{\mathrm{jx}} \mathrm{S}_{\mathrm{kx}}-\left(\mathrm{S}_{\mathrm{j}} \cdot \mathrm{S}_{\mathrm{k}}\right)\right]=\mathrm{P}_{2}(\cos \theta) \mathcal{H}_{\mathrm{S}} .
$$


Note that the effective dipolar frequency $b_{j k}\left(r_{j k}, \beta_{j k}\right)$ now depends on the angle between the internuclear vector and the rotor axis and that the Hamiltonian is proportional to the second-order Legendre polynomial of the cosine of the angle between the rotor and the static magnetic field. In the case of magic-angle spinning, $\mathrm{P}_{2}\left(\cos 54.74^{\circ}\right)$ equals zero, and special recoupling sequences are needed to drive the spin diffusion. ${ }^{252,236}$

\subsection{Reversal of Rf-Driven Spin Diffusion}

\subsubsection{Pulse Sequence and Experimental Apparatus}

The presence of the scaling term $\mathrm{P}_{2}(\cos \theta)$ in Equation (7.4) provides the experimenter with the possibility of switching the sign of the Hamiltonian that governs ifdriven spin diffusion by changing the orientation of the rotor axis relative to $B_{0}$. The rf pulse sequence shown in Figure 7.2 takes advantage of this property and represents a new type of polarization-echo experiment. ${ }^{234,236}$

Hartmann-Hahn cross polarization ${ }^{11,227}$ is used to polarize the S spins during a preparation period $\tau_{\mathrm{CP}}$ while the sample is spun about an axis oriented at the angle $\theta_{1}=35.25^{\circ}$ relative to $B_{0}$. After a frequency-labeling period $t_{1}$, a cw spin-lock is applied. For a time $\tau_{1}$, rf-driven spin diffusion occurs with a scaling factor of $P_{2}\left(\cos 35.25^{\circ}\right)=0.5$. During the time $\tau_{2}$, the sample is rapidly reoriented to $\theta_{2}=90^{\circ}$, and the spins evolve under a driving Hamiltonian with a scaling factor of $P_{2}\left(\cos 90^{\circ}\right)=-0.5$. The signal is then acquired for a time $t_{2}$. It is easily seen that the propagator $\mathrm{e}^{-\mathrm{i} \mathrm{P}_{2}\left(\cos \Theta_{1}\right) \mathcal{H}_{\mathrm{S}} \tau_{1} / \hbar} \mathrm{e}^{-\mathrm{i} \mathrm{P}_{2}\left(\cos \Theta_{2}\right) \mathcal{H}_{\mathrm{S}} \tau_{2} / \hbar}$ is the unity operator if $\tau_{1}=\tau_{2}$, and an echo occurs at that point in time, even for many-body interactions. Obviously, an echo can also be formed with other combinations of $\theta_{1}$ and $\theta_{2}$.

Although a polarization echo could also be observed in a one-dimensional experiment with selective excitation, the two-dimensional version ${ }^{4}$ allows one to distinguish the contributions of spin diffusion from those of $\mathrm{T}_{1 \mathrm{p}}$ relaxation.

To implement the echo pulse sequence of Figure 7.2, a home-built doubleresonance probe in which a stationary coil surrounds a movable stator (see Figure 7.3) was used. A stepper motor connected to a Whedco IMC-1151-1-A controller was used to rapidly reorient the rotor axis during the experiment to within $\pm 0.62^{\circ} .{ }^{10,90}$ Details of the 
probe design, which was originally developed for use in DAS experiments, are described elsewhere. ${ }^{10,155}$ The use of such a probe permitted continuous application of the $\mathrm{rf}$ spin lock during the mechanical hop. It was not possible to avoid irradiating the S-spins during the hop by using $\frac{\pi}{2}$ storage pulses ${ }^{253}$ since the full dipolar order could not be retained. ${ }^{122}$

All spectra in this chapter were recorded on a home-built spectrometer ${ }^{90}$ with a ${ }^{1} \mathrm{H}$ Larmor frequency of $301.2 \mathrm{MHz}$ and a ${ }^{13} \mathrm{C}$ Larmor frequency of $75.7 \mathrm{MHz}$. Adamantane was purchased from Aldrich and used without further purification. The amount of time

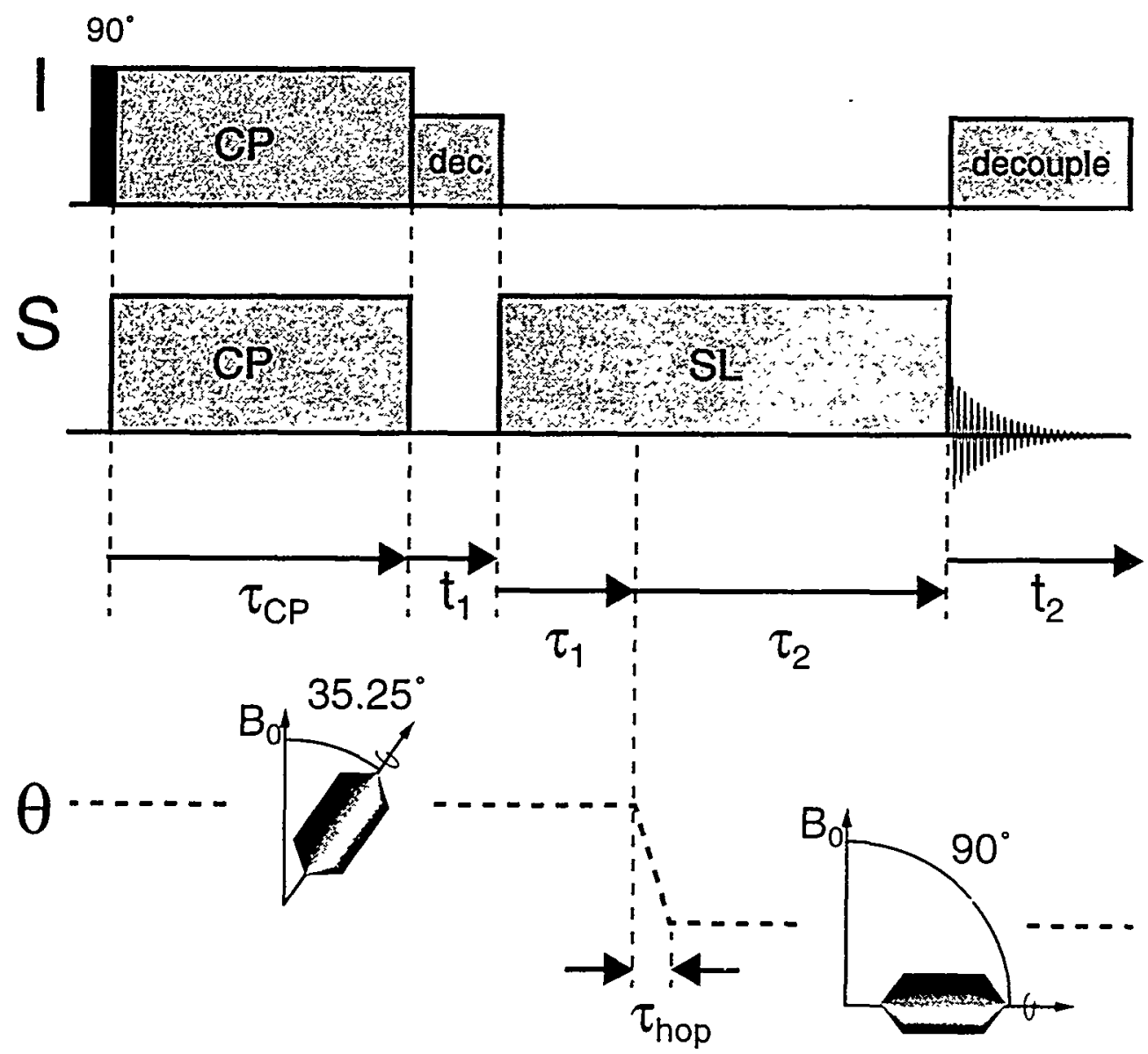

Figure 7.2 - Pulse sequence for refocusing rf-driven spin diffusion by sample reorientation. Hartmann-Hahn cross polarization ${ }^{111,227}$ is used to enhance the polarization of the $S$ spins. The $S$ spins are frequency-labeled during the evolution time, $t_{1}$. For the entire mixing period, spin diffusion among the $S$ spins is driven by a cw spin lock. ${ }^{244,250}$ In the defocusing period, $\tau_{1}$, the sample is spun at the angle $\theta_{1}=35.25^{\circ}$. During the refocusing time, $\tau_{2}$, the rotation axis is rapidly reoriented to $\theta_{2}=90^{\circ}$, and the rf-driven spin diffusion is time reversed at $\tau_{2}=\tau_{1}$. Proton decoupling is applied during both the evolution $\left(t_{1}\right)$ and detection $\left(t_{2}\right)$ periods. 
necessary to reorient the sample was approximately $10 \mathrm{~ms}$. The spinning speed was 5.3 $\mathrm{kHz}$; the if field strengths were $\omega_{1 I^{\prime}} /(2 \pi)=\omega_{1 \mathrm{~s}^{\prime}} /(2 \pi)=20 \mathrm{kHz}$; and the crosspolarization contact time was $5 \mathrm{~ms}$. The ${ }^{13} \mathrm{C}$ carrier frequency was positioned exactly between the two ${ }^{13} \mathrm{C}$ resonances in adamantane for the most efficient chemical-shift scaling during the $\mathrm{cw}$ driven spin diffusion period. ${ }^{244,245,250}$

\subsubsection{Build-up of Cross-Peak Intensity}

The build-up of the cross-peak intensity during the time $\tau_{1}$ with $\tau_{2}=0$ (see Figure 7.2) scales linearly with $\left|P_{2}\left(\cos \theta_{1}\right)\right|$. Here, we interpret the rf-driven spin diffusion as a deterministic quantum-mechanical process described by the Hamiltonian, $\overline{\mathcal{F}}_{\text {Tot }}^{(0)}$ (Equation (7.4)). This can be seen by performing a series expansion about $\tau_{1}=0 .{ }^{7,8}$ Assuming that our system has two distinguishable sites, the intensity of the $\mathrm{A} \rightarrow \mathrm{B}$ cross peak is given by

$$
\begin{aligned}
& \left\langle F_{x}^{B}\left(\tau_{1}\right)\right\rangle=\frac{\operatorname{Tr}\left\{e^{-i \overline{\mathcal{H}}_{\text {fot }}^{(0)} \tau_{1} / \hbar} \rho\left(\tau_{1}=0\right) e^{\mathrm{i} \overline{\mathcal{H}}_{\mathrm{rol}}^{(0)} \tau_{1} / \hbar} F_{x}^{\mathrm{B}}\right\}}{\operatorname{Tr}\left\{\left(\mathrm{F}_{\mathrm{x}}^{\mathrm{B}}\right)^{2}\right\}} \\
& =\sum_{n=0}^{\infty} \frac{(-i)^{n}\left(\tau_{1} P_{2}\left(\cos \theta_{1}\right)\right)^{n}}{n !} M_{n}
\end{aligned}
$$

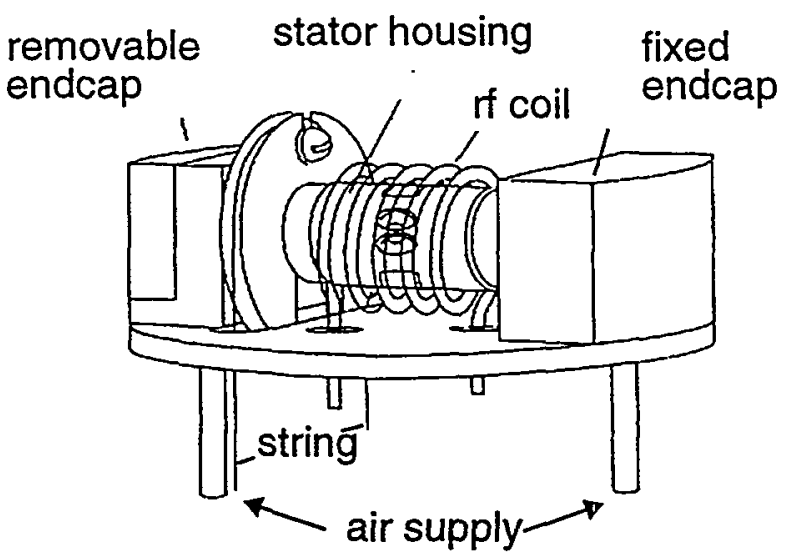

Figure 7.3 - Probe design used in experiments. The stator housing was attached to a pulley which was controlled by a stepper motor so that the rotor angle could be varied during the course of the experiment. ${ }^{10.155}$ The stationary coil permitted rf irradiation to be continuously applied during the reorientation. 
where $F_{x}^{B}$ is the sum magnetization for the spins of type $B$ and the $M_{n}$ denote the moments of the series expansion ${ }^{5,7,8}$

$$
M_{n}=\frac{\left(F_{x}^{B}\left|\left(\hat{\mathcal{H}}_{S} / \hbar\right)^{n}\right| F_{x}^{A}\right)}{\left(F_{x}^{B} \mid F_{x}^{B}\right)}
$$

The scaling behavior can be observed experimentally by performing ${ }^{13} \mathrm{C}$ rf-driven spin diffusion on polycrystalline adamantane for different values of $\mathrm{P}_{2}\left(\cos \theta_{1}\right)$. The largest value for $\left|s b_{j k}\right| /(2 \pi)$ is $11 \mathrm{~Hz}$ for the carbons in natural-abundance adamantane so the condition $\left|s b_{j k}\right| \ll \omega_{r} \ll \omega_{1 s}$ can easily be satisfied. In our experiments, $\omega_{\mathrm{r}} /(2 \pi)=5.3 \mathrm{kHz}$ and $\omega_{1 \mathrm{~s}} /(2 \pi)=20 \mathrm{kHz}$. Figure 7.4 shows the buildup of the normalized cross-peak intensity from rf-driven spin diffusion in adamantane as a function of $\left|P_{2}\left(\cos \theta_{1}\right)\right| \times \tau_{1}$ for three different angles $\theta_{1}$. To within experimental error, all three sets of data points lie on the same curve.

The same scaling behavior results when first-order, time-dependent perturbation theory ${ }^{1,8,23}$ is used to describe the polarization-transfer process. The spin-diffusion rate, $P_{j k}$, between two spins $S_{j}$ and $S_{k}$ in a rapidly rotating sample can be written as ${ }^{1,8}$

$$
P_{j k}\left(\tau_{1}\right)=W_{j k} \tau_{1}=\frac{\pi}{2} s^{2}\left(P_{2}\left(\cos \theta_{1}\right)\right)^{2}\left[b_{j k}\left(r_{j k}, \beta_{j k}\right)\right]^{2} F_{j k}(0) \cdot \tau_{1}
$$

Note that the rate constant was calculated using Fermi's Golden Rule (see Equation (7.1)) and taking into account the sample rotation (see Equation (7.4)). In the ideal case of rfdriven spin diffusion, the abundant I spins are completely decoupled from the S spins and, therefore, $F_{j k}(0)$ scales with $\left|\mathrm{sP}_{2}\left(\cos \Theta_{1}\right) b_{j k}\right|^{-1}$. This leads to

$$
\mathrm{P}_{\mathrm{jk}}\left(\tau_{1}\right) \propto\left|s \mathrm{~b}_{\mathrm{jk}}\right| \cdot\left(\tau_{1}\left|\mathrm{P}_{2}\left(\cos \Theta_{1}\right)\right|\right)
$$

Equations (7.7) and (7.8) predict that the $\mathrm{ff}$-driven spin-diffusion rate constant, $\mathrm{W}_{\mathrm{jk}}$, scales with $1 / r_{j k}^{3}$ in contrast to the proton-driven case where $W_{j k}$ is proportional to $1 / r_{j k}^{6} \cdot{ }^{245,246}$ 
It is interesting to compare the experimental polarization-transfer dynamics with statistical approaches. The three curves shown in Figure 7.4 result from a phenomenological, ad-hoc model of the spin-diffusion process in natural-abundance ${ }^{13} \mathrm{C}$ adamantane assuming a master equation for the polarization $\left(\mathrm{p}_{\mathrm{i}}=\left\langle\mathrm{S}_{\mathrm{ix}}\right\rangle\right)$ exchange

$$
\frac{\mathrm{d}}{\mathrm{dt}} \mathbf{p}=\tilde{\mathbf{w}} \cdot \mathbf{p}
$$

$\tilde{\mathbf{W}}$ represents the polarization-exchange matrix where $\mathrm{W}_{\mathrm{jk}}$ is evaluated according to Equation (7.7) with $\theta_{1}=0^{\circ}$ and the diagonal elements are defined as $W_{j j}=-\sum_{k \neq j} W_{k j}$ to conserve the sum polarization. ${ }^{4}$ An fcc lattice of 1000 adamantane molecules was

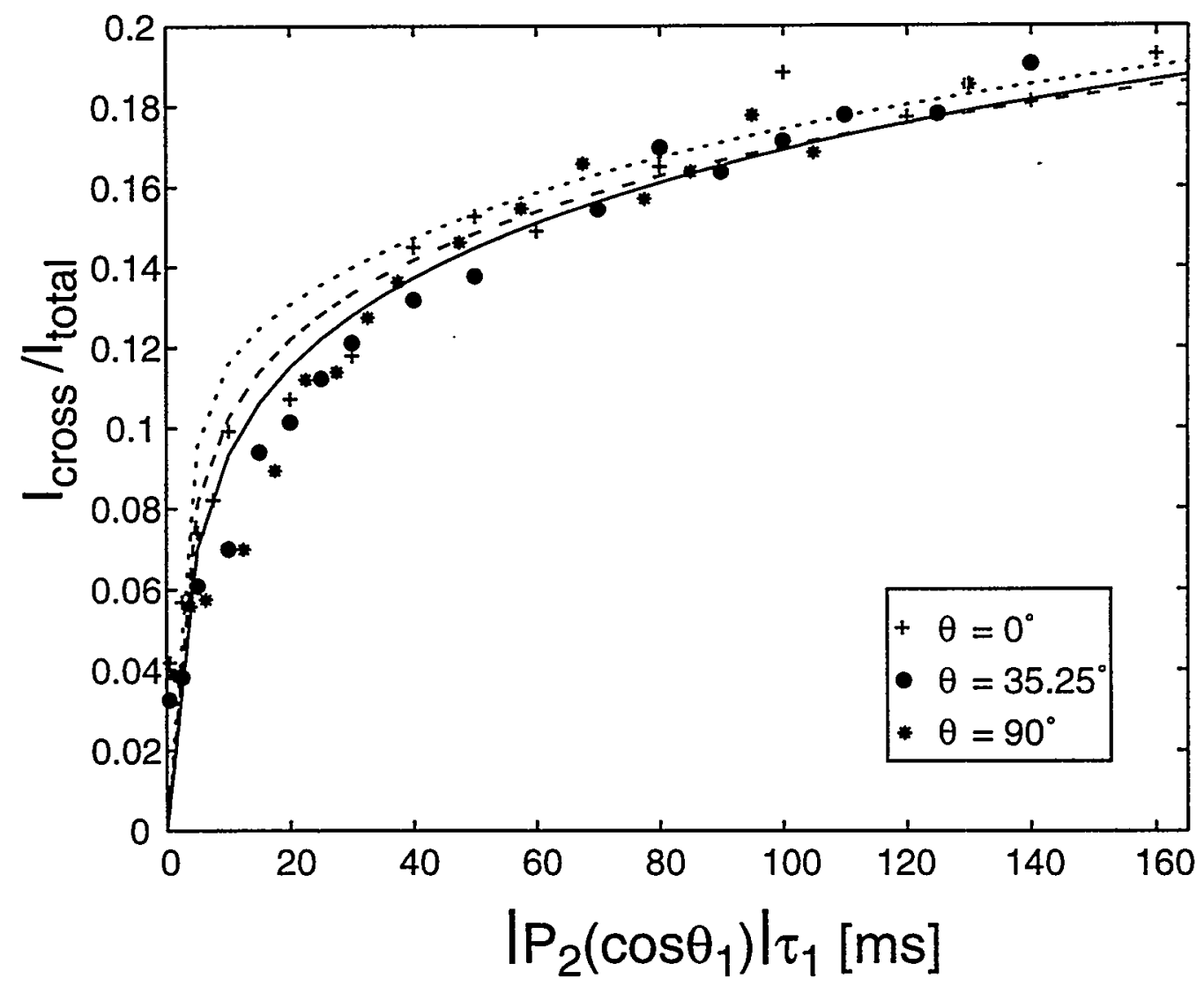

Figure 7.4 - Experimental build-up of the normalized rf-driven spin diffusion cross-peak intensity in ${ }^{13} \mathrm{C}$ natural-abundance adamantane as a function of $\left|P_{2}\left(\cos \theta_{1}\right) \times \tau_{1}\right|$ for $\theta_{1}=0^{\circ}$, $35.25^{\circ}$, and $90^{\circ}$. The curves were numerically calculated from a kinetic master equation for the polarization exchange process (Equation (7.9)) and represent three different model assumptions used for the evaluation of $\mathrm{W}_{\mathrm{jk}}$ as described in the text. Qualitative agreement with the experimental data is obtained when a uniform and constant normalized zeroquantum intensity of $F_{j k}(0)=F(0)=(7 \pm 2) \times 10^{-2} s$ is assumed. 
constructed with a nearest distance of $6.6 \AA$ between molecular centers. ${ }^{254}$ Due to the fast rotational dynamics of adamantane molecules at $300 \mathrm{~K}$, the intramolecular dipolar interactions are averaged to zero, and the observed polarization transfer reflects only intermolecular dipolar interactions. The S-spin lattice sites were occupied by using a random number generator and considering the probabilities of ${ }^{13} \mathrm{CH}_{2}$ and ${ }^{13} \mathrm{CH}$ occurrence at natural abundance. ${ }^{255}$ It was assumed that no more than two ${ }^{13} \mathrm{C}$ atoms were present in a single molecule. The matrix elements, $W_{j k}$, of $\tilde{\mathbf{W}}$ were evaluated separately for each dipolar-coupled spin pair using Equation (7.7) with $\theta_{1}=0^{\circ}$.

The three curves in Figure 7.4 represent three different model assumptions used for the evaluation of $\mathrm{W}_{\mathrm{jk}}$. The dotted curve was generated using the approximation that $\left\langle\left(1-3 \cos ^{2} \beta_{\mathrm{jk}}\right)^{2}\right\rangle_{\text {powder }}=4 / 5$ for each spin pair. ${ }^{8}$ The dashed curve was obtained by performing an explicit powder average over 1000 orientations using the method of Cheng et $a .^{38}$ (see Section 1.3). The solid curve was obtained by additionally taking into account the fast rotational diffusion of the adamantane molecules on their lattice sites which leads to a motionally averaged internuclear distance $\left\langle\mathrm{r}_{\mathrm{jk}}\right\rangle$ and angle $\left\langle\beta_{\mathrm{jk}}\right\rangle .{ }^{255}$ All three curves represent an average over 100 different randomly occupied S-spin lattices. Qualitative agreement with the experimental data is obtained when a uniform and constant normalized zero-quantum intensity of $F_{j k}(0)=F(0)=(7 \pm 2) \times 10^{-2} s$ is assumed. This leads to a linewidth of the normalized $S$ spin zero-quantum spectrum of $10-14 \mathrm{~Hz}$ assuming a Lorentzian or Gaussian shape and agrees well with the strength of the ${ }^{13} \mathrm{C}-{ }^{13} \mathrm{C}$ dipolar couplings in natural-abundance adamantane $\left(\left|\mathrm{sb}_{\mathrm{jk}}^{\max }\right| /(2 \pi)=11 \mathrm{~Hz}\right)$.

The time dependence of the polarization transfer is clearly non-exponential due to the statistical distribution of the ${ }^{13} \mathrm{C}$ spins on the lattice sites. For $\tau_{1} \leq 30 \mathrm{~ms}$, the ${ }^{13} \mathrm{CH}$ and ${ }^{13} \mathrm{CH}_{2}$ pairs on neighboring molecules predominantly contribute to the cross-peak intensities. For longer times, more remote spin packets (within the next nearest neighbor shell for the plotted time range) start to contribute as well, leading to a flattening of the build-up curve. Due to the isotopic dilution and the crystal structure of adamantane, the spin-diffusion dynamics appear to follow the predictions made for coupled clusters of spins. ${ }^{177}$ 


\subsubsection{Spin-Diffusion Echoes}

Figure 7.5 shows a set of three two-dimensional spectra of adamantane obtained with $\tau_{1}=30 \mathrm{~ms}$ and different values of $\tau_{2}$. In Figure $7.5 \mathrm{a}, \tau_{2}=0 \mathrm{~ms}$ and the sample was spun at $\theta_{1}=35.25^{\circ}$. Rf-driven spin diffusion proceeded according to the Hamiltonian of Equation (7.4) with the scaling factor $P_{2}\left(\cos \theta_{1}\right)=0.5$, and the spin-diffusion cross peaks are clearly visible. Figure $7.5 \mathrm{~b}$ shows the spectrum corresponding to the polarization echo. In this experiment, the S-spin system evolved under a Hamiltonian with a scaling factor of $P_{2}\left(\cos \theta_{1}\right)=0.5$ for $\tau_{1}=30 \mathrm{~ms}$. The sample was then reoriented, and the evolution continued with a scaling factor of $P_{2}\left(\cos \theta_{2}\right)=-0.5$ for $\tau_{2}=50 \mathrm{~ms}$. The opposite signs of the scaling factor during $\tau_{1}$ and $\tau_{2}$ caused the evolution of the polarization transfer to refocus, and the cross-peak intensity approached zero. Since a finite time was required for the sample reorientation, the polarization echo was delayed and occurred at $\tau_{2} \approx 1.3 \tau_{1}$. Figure $7.5 \mathrm{c}$ shows the case where $\tau_{2}$ was much greater than $\tau_{1}$. In this spectrum, the cross-peak intensity has recovered and reached a value exceeding that shown in Figure 7.5a.

The complete time evolution of the echo is depicted in Figure 7.6. The normalized cross-peak intensities are plotted as a function of total mixing time for $\tau_{1}=30 \mathrm{~ms}$ (Figure 7.6a) and $\tau_{1}=70 \mathrm{~ms}$ (Figure 7.6b). For the experiments with the shorter $\tau_{1}$ time, the refocusing of the spin diffusion is nearly complete, but at longer times the echo, though sharp, is weaker in amplitude. The reason for this is unclear. One possibility is that the strength of the ${ }^{13} \mathrm{C}$ rf field used in these experiments $\left(\omega_{1 S} /(2 \pi)=20 \mathrm{kHz}\right)$ may not be sufficient to fully decouple the abundant proton spins. The residual heteronuclear dipolar coupling Hamiltonian, $\mathcal{H}_{\mathrm{IS}}$, and the homonuclear dipolar coupling among the I spins, $\mathscr{H}_{\mathrm{II}}$, will not be inverted by the sample reorientation since the spinning speed $\left(\omega_{\mathrm{r}} /(2 \pi)=5.3\right.$ $\mathrm{kHz}$ ) is not fast compared to these interactions. Consequently, the S-spin polarization echo amplitude will be damped. The flatness of the echo peak in Figure 7.6a is due to the finite time required for sample reorientation. The evolution during the reorientation is difficult to quantify since it is not known precisely how much time the sample spends at each angle. Furthermore, the scaling factor, $\mathrm{P}_{2}(\cos \theta)$, varies non-linearly with $\theta$, and the 
a)

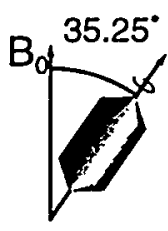

b)

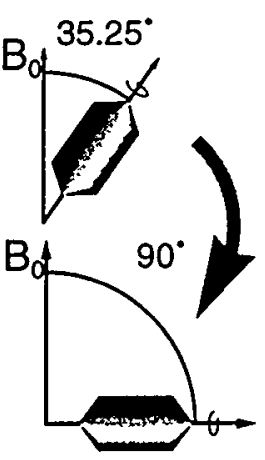

c)

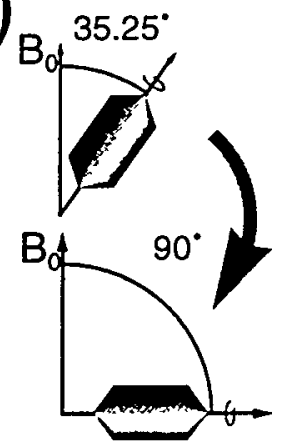

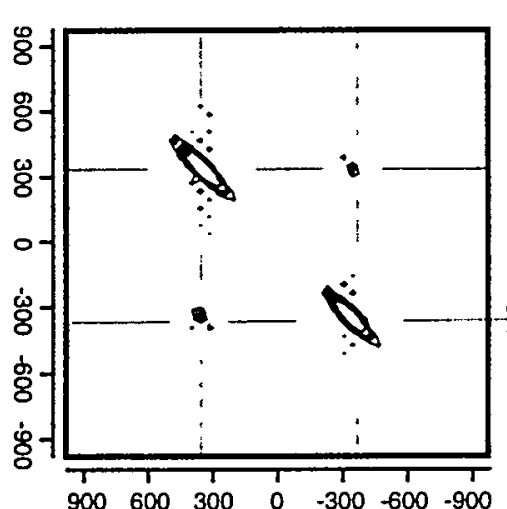

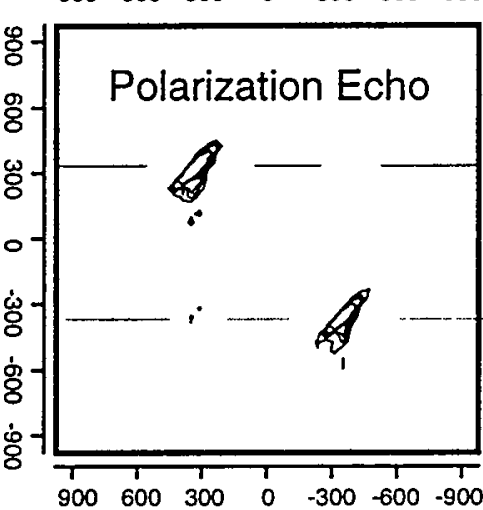

尌

$\begin{array}{lllllll}900 & 600 & 300 & 0 & -300 & -600 & -900\end{array}$

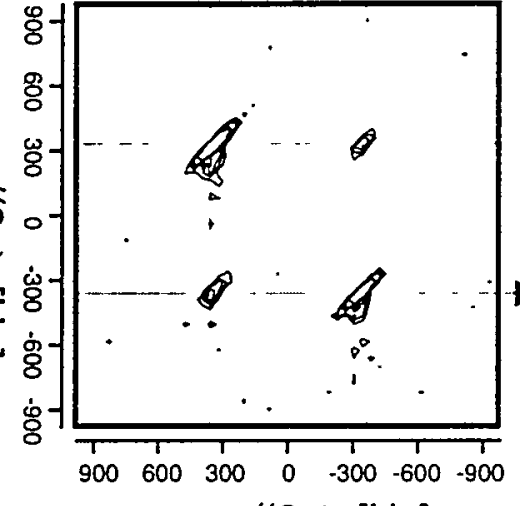

$\tau_{1}=30 \mathrm{~ms}$

$\tau_{2}=0 \mathrm{~ms}$

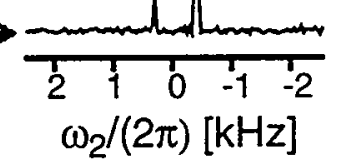

$\tau_{1}=30 \mathrm{~ms}$

$\tau_{2}=50 \mathrm{~ms}$

$$
\tau_{1}=30 \mathrm{~ms}
$$

$\tau_{2}=110 \mathrm{~ms}$

$\rightarrow \frac{1}{\frac{1}{2} \omega_{0} /(2 \pi)[\mathrm{kHz}]}$

$\omega_{2} /(2 \pi)[\mathrm{Hz}]$

Figure 7.5 - Experimental two-dimensional ${ }^{13} \mathrm{C}$ rf-driven spin diffusion spectra of adamantane. For the two-dimensional spectra, 90 complex $t_{1}$ points consisting of 16 scans in each were collected according to the method of States et al. ${ }^{51}$ The delay between experiments was $3.5 \mathrm{~s}$. (a) Rf-driven spin diffusion spectrum obtained at $\theta_{1}=35.25^{\circ}$ (no axis reorientation) with a mixing time of $\tau_{1}=30 \mathrm{~ms}$. Cross peaks due to spin diffusion are clearly visible. (b) Echo experiment obtained using the pulse sequence of Figure 7.2 with $\tau_{1}=30 \mathrm{~ms}$ and $\tau_{2}=50 \mathrm{~ms}$. The spin diffusion has been refocused, and the cross-peak intensity is nearly zero. Note that the orientation of the diagonal peaks has changed due to the sign change of $P_{2}(\cos \theta)$ (which affects residual chemical-shift, dipolar, and bulk susceptibility interactions during the evolution and detection periods). (c) Experiment obtained using the pulse sequence of Figure 7.2 with $\tau_{1}=30 \mathrm{~ms}$ and $\tau_{2}=110 \mathrm{~ms}$. The longer evolution at the second angle led to a recovery of the cross-peak intensities. In all three spectra, the contours are at $3,5,7,9,11,13$, and $15 \%$ of the maximum signal intensity. 

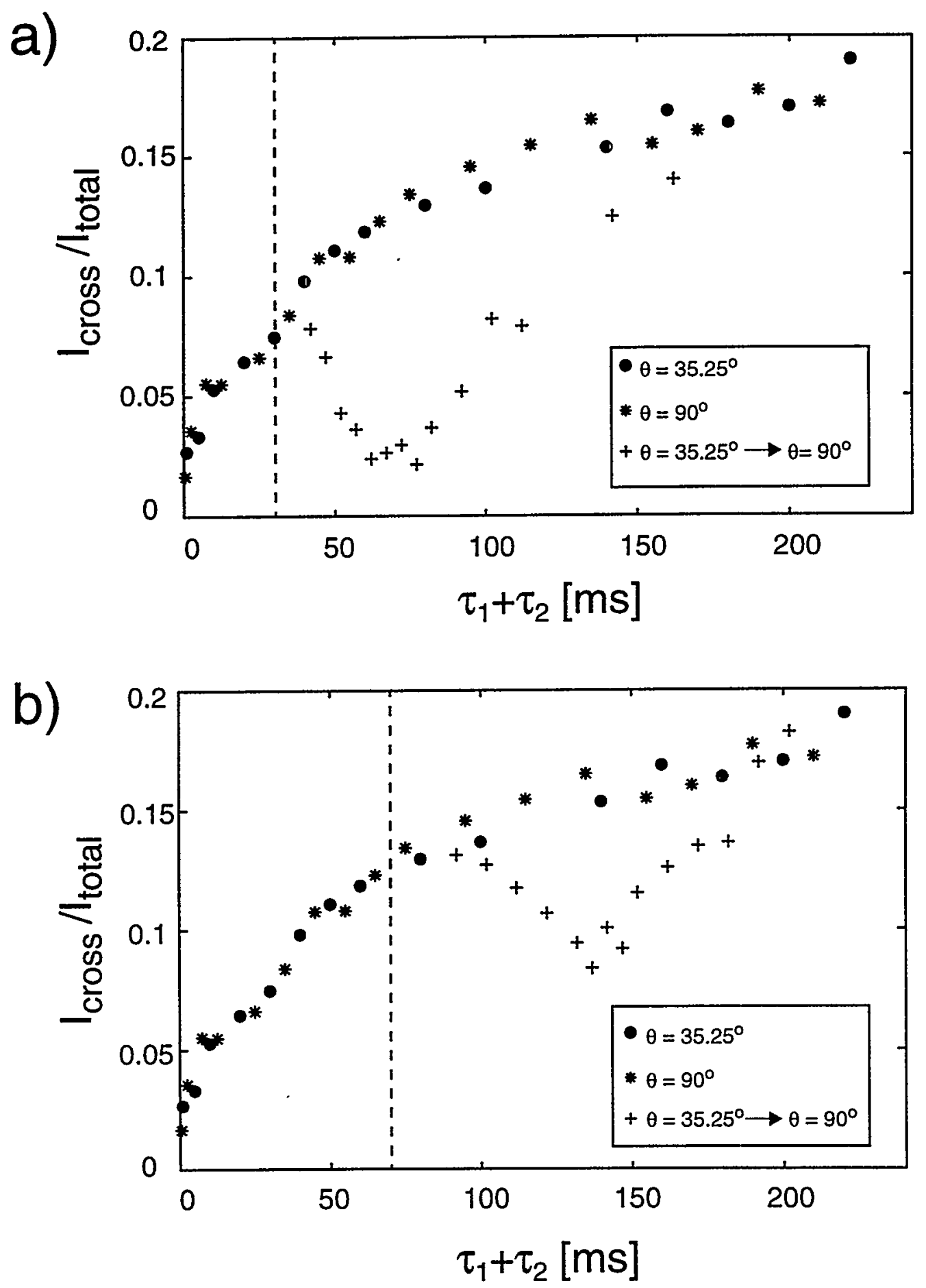

Figure 7.6 - Time evolution of the normalized cross-peak intensity in adamantane. The circles and asterisks show the cross-peak build-up as a function of mixing time for rfdriven spin diffusion at angles of $\theta_{1}=35.25^{\circ}$ and $90^{\circ}$, respectively. The crosses show the cross-peak intensities as a function of time for the echo experiment of Figure 7.2 with (a) $\tau_{1}=30 \mathrm{~ms}$ and (b) $\tau_{1}=70 \mathrm{~ms}$. The time at which the hop is initiated is indicated by a vertical line in each graph. 
sign change occurs at an angle $\left(\theta=54.74^{\circ}\right)$ that is closer to $35.25^{\circ}$ than to $90^{\circ}$. For these reasons, the echo position will be sensitive to instabilities in the mechanical reorientation process, leading to a broadening of the echo maximum for $\tau_{1} \approx \tau_{\text {hop }}$.

In summary, a new type of polarization echo has been introduced. We have experimentally demonstrated that the spin-diffusion process can be refocused by a mechanical sample reorientation. Rf-driven ${ }^{13} \mathrm{C}$ polarization echoes were observed for mixing times on the order of $100 \mathrm{~ms}$, which is more than two orders of magnitude longer than the time scale for previously observed proton dipolar echoes. ${ }^{234,236}$ Although rfdriven spin diffusion in adamantane can be qualitatively described by a master equation for polarization exchange, such an approach obviously fails to describe the formation of the echoes. 


\section{References}

(1) J. J. Sakurai, Modern Quantum Mechanics (Addison-Wesley Publishing Company, Inc., Redwood City, CA, 1985).

(2) C. Cohen-Tannoudji, B. Diu and F. Laloë, Quantum Mechanics (John Wiley and Sons, New York, 1977).

(3) P. A. M. Dirac, The Principles of Quantum Mechanics (Clarendon Press, Oxford, England, 1958).

(4) R. R. Ernst, G. Bodenhausen and A. Wokaun, Principles of Nuclear Magnetic Resonance in One and Two Dimensions (Clarendon Press, Oxford, England, 1987).

(5) C. P. Slichter, Principles of Magnetic Resonance (Springer-Verlag, Berlin, Germany, 1990).

(6) U. Haeberlen, High Resolution NMR in Solids - Selective Averaging (Academic Press, New York, NY, 1976).

(7) M. Mehring, Principles of High-Resolution NMR in Solids (Springer-Verlag, Berlin, Germany, 1976).

(8) A. Abragam, Principles of Nuclear Magnetism (Clarendon Press, Oxford, England, 1961).

(9) M. Munowitz, Coherence and NMR (Wiley, New York, NY, 1988).

(10) K. T. Mueller, Ph.D. Thesis, University of California, Berkeley, 1991.

(11) J. H. Baltisberger, Ph.D. Thesis, University of California, Berkeley, 1993.

(12) U. Fano, Rev. Mod. Phys. 29, 74 (1957).

(13) F. Dyson, Phys. Rev. 75, 486 (1949).

(14) F. Dyson, Phys. Rev. 75, 1736 (1949).

(15) J. Cavanagh, W. J. Fairbrother, A. G. P. II and N. J. Skelton, Protein NMR Spectroscopy (Academic Press, Inc., San Diego, 1996).

(16) M. E. Rose, Elementary Theory of Angular Momentum (John Wiley \& Sons, New York, NY, 1957).

(17) A. R. Edmonds, Angular Momentum in Quantum Mechanics (Princeton University Press, Princeton, NJ, 1960). 
(18) G. Arfken, Mathematical Methods for Physicists (Academic Press, Inc., San Diego, CA, 1985).

(19) A. Schmidt and S. Vega, J. Chem. Phys. 96, 2655 (1992).

(20) O. Weintraub and S. Vega, J. Magn. Reson., Ser. A 105, 245 (1993).

(21) A. E. Bennett, R. G. Griffin and S. Vega, NMR Basic Principles Progr. 33, 3 (1994).

(22) T. O. Levante, M. Baldus, B. H. Meier and R. R. Ernst, Mol. Phys. 86, 1195 (1995).

(23) K. Schmidt-Rohr and H. W. Spiess, Multidimensional Solid-State NMR and Polymers (Academic Press Inc., San Diego, 1994).

(24) T. M. Duncan, A Compilation of Chemical Shift Anisotropies (The Farragut Press, Chicago, 1990).

(25) T. P. Das and E. L. Hahn, Nuclear Quadrupole Resonance Spectroscopy (Academic Press, New York, NY, 1958).

(26) M. H. Cohen and F. Reif In Solid State Phys.; F. Seitz and D. Turnbull, Eds., Academic Press, New York, 1957, Vol. 5.

(27) D. Freude and J. Haase, NMR Basic Principles Progr. 29, 1 (1993).

(28) M. Goldman, P. J. Grandinetti, A. Llor, Z. Olejniczak, J. R. Sachleben and J. W. Zwanziger, J. Chem. Phys. 97, 8947 (1992).

(29) C. H. Townes and B. P. Dailey, J. Chem. Phys. 17, 782 (1949).

(30) E. A. C. Lucken, Nuclear Quadrupole Coupling Constants (Academic Press, London, 1969).

(31) P. J. Grandinetti, J. H. Baltisberger, I. Farnan, J. F. Stebbins, U. Werner and A. Pines, J. Phys. Chem. 99, 12341 (1995).

(32) C. A. Fyfe, H. Gies and Y. Feng, J. Am. Chem. Soc. 111, 7702 (1989).

(33) C. A. Fyfe, H. Grondey, Y. Feng and G. T. Kokotailo, Chem. Phys. Lett. 173, 211 (1990).

(34) C. A. Fyfe, Y. Feng, H. Gies, H. Grondey and G. T. Kokotailo, J. Am. Chem. Soc. 112, 3264 (1990).

(35) C. A. Fyfe, H. Gies, Y. Feng and G. T. Kokotailo, Nature 341, 223 (1989).

(36) C. A. Fyfe, H. Grondey, Y. Feng and G. T. Kokotailo, J. Am. Chem. Soc. 112, 8812 (1990). 
(37) D. W. Alderman, M. S. Solum and D. M. Grant, J. Chem. Phys. 84, 3717 (1986).

(38) V. B. Cheng, J. Henry H. Suzukawa and M. Wolfsberg, J. Chem. Phys. 59, 3992 (1973).

(39) M. M. Maricq and J. S. Waugh, J. Chem. Phys. 70, 3300 (1979).

(40) J. Herzfeld and A. E. Berger, J. Chem. Phys. 73, 6021 (1980).

(41) D. M. Brink and G. R. Satchler, Angular Momentum (Oxford University Press, Inc., New York, 1993).

(42) L. Frydman and J. S. Harwood, J. Am. Chem. Soc. 117, 5367 (1995).

(43) A. Medek, J. S. Harwood and L. Frydman, J. Am. Chem. Soc. 117, 12779 (1995).

(44) K. Schmidt-Rohr, M. Hehn, D. Schaefer and H. W. Spiess, J. Chem. Phys. 97, 2247 (1992).

(45) J. Keeler In Multinuclear Magnetic Resonance in Liquids and Solids -- Chemical Applications; P. Granger and R. K. Harris, Eds., Kluwer Academic Publishers, Dordrecht, The Netherlands, 1990, .

(46) D. I. Hoult and R. E. Richards, Proc. Roy. Soc. (London) A344, 311 (1975).

(47) E. O. Stejskal and J. Schaefer, J. Magn. Reson. 18, 560 (1975).

(48) J. Keeler and D. Neuhaus, J. Magn. Reson. 63, 454 (1985).

(49) G. Drobny, A. Pines, S. Sinton, D. Weitekamp and D. Wemmer, Symp. Faraday Soc. 13, 49 (1979).

(50) D. Marion and K. Wüthrich, Biochem. Biophys. Res. Commun. 113, 967 (1983).

(51) D. J. States, R. A. Haberkorn and D. J. Ruben, J. Magn. Reson. 48, 286 (1982).

(52) A. Bax, A. F. Mehlkopf and J. Smidt, J. Magn. Reson. 35, 373 (1979).

(53) D. Massiot, B. Touzo, D. Trumeau, J. P. Coutures, J. Virlet, P. Florian and P. J. Grandinetti, Solid State NMR 6, 73 (1996).

(54) P. J. Grandinetti, J. H. Baltisberger, A. Llor, Y. K. Lee, U. Werner, M. A. Eastman and A. Pines, J. Magn. Reson., Ser. A 103, 72 (1993).

(55) P. J. Grandinetti, Y. K. Lee, J. H. Baltisberger, B. Q. Sun and A. Pines, J. Magn. Reson., Ser. A 102, 195 (1993).

(56) K. T. Mueller, E. W. Wooten and A. Pines, J. Magn. Reson. 92, 620 (1991). 
(57) J. M. O'Connor and C. P. Casey, Chem. Rev. 87, 307 (1987).

(58) D. E. Shriver, P. W. Atkins and C. H. Langford, Inorganic Chemistry (W. H. Freeman and Company, New York, 1990).

(59) T. S. Piper and G. J. Wilkinson, J. Inorg. Nucl. Chem. 2, 38 (1956).

(60) F. A. Cotton In Dynamic Nuclear Magnetic Spectrsocopy; L. M. Jackman and F. A. Cotton, Eds., Academic Press, New York, 1975, .

(61) R. Benn, H. Grondey, R. Nolte and G. Ecker, Organometallics 7, 777 (1988).

(62) R. B. Woodward and R. Hoffman, The Conservation of Orbital Symmetry (Verlag Chemie GmbH, Weinheim, Germany, 1970).

(63) A. Liberles, Introduction to Molecular Orbital Theory (Holt, Rinehard, and Winston Inc., New York, 1966).

(64) Y. Jean, F. Volatron and J. Burdett, An Introduction to Molecular Orbitals (Oxford University Press Inc., New York, 1993).

(65) S. M. Owen and A. T. Brooker, A Guide to Modern Inorganic Chemistry (Longman Group UK Limited, London, 1991).

(66) R. B. Jordan, Reaction Mechanisms of Inorganic and Organometallic Systems (Oxford University Press, Inc., New York, 1991).

(67) B. E. Mann In Comprehensive Organometallic Chemistry; G. Wilkinson, F. G. A. Stone and E. W. Abel, Eds., Pergamon Press Ltd., Oxford, 1982, Vol. 3.

(68) C.-C. Su, J. Am. Chem. Soc. 93, 5653 (1970).

(69) G. M. Whitesides and J. S. Fleming, J. Am. Chem. Soc. 89, 2855 (1967).

(70) F. A. Cotton, Advanced Inorganic Chemistry (John Wiley and Sons, New York, 1988).

(71) F. A. Cotton and T. J. Marks, J. Am. Chem. Soc. 91, 3178 (1969).

(72) A. J. Campbell, C. A. Fyfe and J. E. Maslowsky, J. Am. Chem. Soc. 94, 2690 (1972).

(73) C. E. Cottrell, C. A. Fyfe and C. V. Senoff, J. Organometal. Chem. 43, 203 (1972).

(74) J. R. Lyerla, C. S. Yannoni and C. A. Fyfe, Acc. Chem. Res. 15, 208 (1982).

(75) S. E. Anderson, J. Organomet. Chem. 71, 263 (1974).

(76) A. J. Campbell, C. A. Fyfe, R. G. Goel, J. E. Maslowsky and C. V. Senoff, J. Am. Chem. Soc. 94, 8387 (1972). 
(77) A. J. Campbell, C. E. Cottrell, C. A. Fyfe and K. R. Jeffrey, Inorg. Chem. 15, 1326 (1976).

(78) D. E. Wemmer, D. J. Reuben and A. Pines, J. Am. Chem. Soc. 103, 28 (1981).

(79) B. E. Hanson, E. C. Lisic, J. T. Petty and G. A. Iannaconne, Inorg. Chem. 25, 4062 (1986).

(80) B. E. Hanson and E. C. Lisic, Inorg. Chem. 25, 716 (1986).

(81) G. W. Wagner and B. E. Hanson, Inorg. Chem. 1987, 2019 (1987).

(82) S. J. Heyes and C. M. Dobson, J. Am. Chem. Soc. 113, 463 (1991).

(83) R. Benn, H. Grondey, G. Erker, R. Aul and R. Nolte, Organometallics 9, 2493 (1990).

(84) R. Benn, R. Mynott, I. Topalovic and F. Scott, Organometallics 8, 2299 (1989).

(85) J. Kuemmerlen and A. Sebald, J. Am. Chem. Soc. 115, 1134 (1993).

(86) V. I. Kulishov, N. G. Bokii, A. F. Prikhot'ko and Y. T. Struchkov, Zh. Strukt. Khim. 16, 252 (1975).

(87) R. D. Rogers, R. V. Bynum and J. L. Atwood, J. Am. Chem. Soc. 103, 692 (1981).

(88) R. D. Rogers, R. V. Bynum and J. L. Atwood, J. Am. Chem. Soc. 100, 5238 (1978).

(89) C. M. Lukehart, Fundamental Transition Metal Organometallic Chemistry (Wadsworth, Inc., Belmont, CA, 1985).

(90) Y. K. Lee, Ph.D. Thesis, University of California, Berkeley, 1994.

(91) F. D. Doty, R. R. Inners and P. D. Ellis, J. Magn. Reson. 43, 399 (1981).

(92) E. O. Stejskal, J. Schaefer and J. S. Waugh, J. Magn. Reson. 28, 105 (1977).

(93) G. C. Campbell, R. C. Crosby and J. F. Haw, J. Magn. Reson. 69, 191 (1986).

(94) J. F. Haw, G. C. Campbell and R. C. Crosby, Anal. Chem. 58, 3172 (1986).

(95) G. Allen, Ph.D. Thesis, University of Sydney, 1989.

(96) H.-O. Kalinowski, S. Berger and S. Braun, ${ }^{13}$ C NMR Spectroscopy (John Wiley and Sons, New York, 1988).

(97) V. I. Kulishov, E. M. Brainina, N. G. Bokiy and Y. T. Struchkov, J. Organomet. Chem. 36, 333 (1972).

(98) J. L. Calderon, F. A. Cotton and J. Takatas, J. Am. Chem. Soc. 93, 3587 (1971). 
(99) H. J. M. de Groot, S. O. Smith, A. C. Kolbert, J. M. L. Courtin, C. Winkel, J. Lugtenburg, J. Herzfeld and R. G. Griffin, J. Magn. Reson. 91, 30 (1991).

(100) W. P. Rothwell and J. S. Waugh, J. Chem. Phys. 74, 2721 (1981).

(101) W. S. Veeman, Progr. NMR Spectroscop. 16, 193 (1984).

(102) V. I. Kulishov, E. M. Brainina, N. G. Bokiy and Y. T. Struchkov, Chem. Comm. 475 (1970).

(103) J. L. Calderon, F. A. Cotton, B. G. DeBoer and J. Takats, J. Am. Chem. Soc. 93, 3592 (1971).

(104) D. L. Kepert, The Early Transition Metals (Academic Press, Inc., London, 1972).

(105) K. Takegoshi and C. A. McDowell, J. Am. Chem. Soc. 108, 6852 (1986).

(106) C. Conner, A. Naito, K. Takegoshi and C. A. McDowell, Chem. Phys. Lett. 113, 123 (1985).

(107) D. L. VanderHart, J. Magn. Reson. 72, 13 (1987).

(108) H.-H. Limbach, B. Wehrle, M. Schlabach, R. Kendrick and C. S. Yannoni, J. Magn. Reson. 77, 84 (1988).

(109) C. A. Fyfe, Solid State NMR for Chemists (C.F.C. Press, Guelph, 1983).

(110) G. Engelhardt and H. Koller In Solid State NMR II: Inorganic Matter; B. Blümich, Ed. Springer-Verlag, Berlin, 1994, Vol. 31.

(111) S. R. Hartmann and E. L. Hahn, Phys. Rev. 128, 2042 (1962).

(112) G. E. Harlow and J. G. E. Brown, Am. Mineral. 65, 986 (1980).

(113) R. J. Kirkpatrick, R. A. Kinsey, K. A. Smith, D. M. Henderson and E. Oldfield, Am. Mineral. 70, 106 (1985).

(114) K. A. Smith, R. J. Kirkpatrick, E. Oldfield and D. M. Henderson, Am. Mineral. 68, 1206 (1983).

(115) D. E. Woessner and J. C. Trewella, J. Magn. Reson. 59, 352 (1984).

(116) E. Lippmaa, M. Mägi, A. Samoson, G. Engelhardt and A.-R. Grimmer, J. Am. Chem. Soc. 102,4889 (1980).

(117) J. V. Smith, C. S. Blackwell and G. L. Hovis, Nature 309, 140 (1984).

(118) D. E. Woessner and H. K. C. Timken, J. Magn. Reson. 90, 411 (1990). 
(119) E. Brun, S. Hafner and P. Hartmann, Helv. Phys. Acta 33, 495 (1960).

(120) A. J. Vega, J. Magn. Reson. 96, 50 (1992).

(121) A. J. Vega, Solid State NMR 1, 17 (1992).

(122) J. H. Baltisberger, S. L. Gann, P. J. Grandinetti and A. Pines, Mol. Phys. 81, 1109 (1994).

(123) W. Sun, J. T. Stephen, L. D. Potter and Y. Wu, J. Magn. Reson., Ser. A 116, 181 (1995).

(124) E. R. Andrew, Int. Rev. Phys. Chem. 1, 195 (1981).

(125) L. Müller, Chem. Phys. 61, 235 (1981).

(126) S. Hayashi, Solid State NMR 3, 93 (1994).

(127) L. van Wüllen, L. Züchner, W. Müller-Warmuth and H. Eckert, Solid State NMR 6, 203 (1996).

(128) E. Fukushima and S. B. W. Roeder, Experimental Pulse NMR: A Nuts and Bolts Approach (Addison-Wesley Publishing Company, Inc., Reading, MA, 1981).

(129) J. Heller, Ph.D. Thesis, University of California, 1997.

(130) S. A. Smith, T. O. Levante, B. H. Meier and R. R. Ernst, J. Magn. Reson., Ser. A 106, 75 (1994).

(131) Z. Gan, D. M. Grant and R. R. Ernst, Chem. Phys. Lett. 254, 349 (1996).

(132) J. Seliger, J. Magn. Reson., Ser. A 116, 67 (1995).

(133) J. Haase and E. Oldfield, Solid-State Nuclear Magnetic Resonance 3, 171 (1994).

(134) C. A. Fyfe, K. C. Wong-Moon, Y. Huang, H. Grondey and K. T. Mueller, J. Phys. Chem. 99, 8707 (1995).

(135) T. H. Walter, G. L. Turner and E. Oldfield, J. Magn. Reson. 76, 106 (1988).

(136) S. Vega, Phys. Rev. A 23, 3152 (1981).

(137) S. Vega, T. W. Shattuck and A. Pines, Phys. Rev. A 22, 638 (1980).

(138) P. Brunner, M. Reinhold and R. R. Ernst, Journal of Chemical Physicss 73, 1086 (1980).

(139) C. S. Blackwell and R. L. Patton, J. Phys. Chem. 88, 6135 (1984). 
(140) R. G. Bryant, S. Ganapathy and S. D. Kennedy, J. Magn. Reson. 72, 376 (1987).

(141) R. K. Harris and G. Nesbitt, J. Magn. Reson. 78, 245 (1988).

(142) C. A. Fyfe, H. Grondey, K. T. Mueller, K. C. Wong-Moon and T. Markus, J. Am. Chem. Soc. 114, 5876 (1992).

(143) C. A. Fyfe, K. T. Mueller, H. Grondey and K. C. Wong-Moon, J. Phys. Chem. 97, 13484 (1993).

(144) C. A. Fyfe, K. C. Wong-Moon, H. Grondey and K. T. Mueller, J. Phys. Chem. 98, 2139 (1994).

(145) W. Kolodziejski and A. Corma, Solid State NMR 3, 177 (1994).

(146) The ARRL Handbook for Radio Amateurs (The American Radio Relay League, Newington, CT, 1994).

(147) A. Verhoeven, R. Verel and B. H. Meier, Chem. Phys. Lett. 266, 465 (1997).

(148) M. H. Levitt, D. Suter and R. R. Ernst, J. Chem. Phys. 84, 4243 (1986).

(149) D. Michel and F. Engelke, NMR Basic Principles Progr. 32, 69 (1994).

(150) B. H. Meier, Chem. Phys. Lett. 188, 201 (1992).

(151) D. Marks and S. Vega, J. Magn. Reson., Ser. A 118, 157 (1996).

(152) D. E. Demco, J. Tegenfeld and J. S. Waugh, Phys. Rev. B 11, 4133 (1975).

(153) J. S. Shore, S. M. De Paul, M. Ernst, J. F. Stebbins and A. Pines, to be published.

(154) E. O. Stejskal, J. Schaefer and R. A. McKay, J. Magn. Reson. 57, 471 (1984).

(155) K. T. Mueller, G. C. Chingas and A. Pines, Rev. Sci. Instrum. 62, 1445 (1991).

(156) M. Tomaselli, B. H. Meier, M. Baldus, J. Eisenegger and R. R. Ernst, Chem. Phys. Lett. 225, 131 (1994).

(157) S. Zhang, J. Magn. Reson., Ser. A 110, 73 (1994).

(158) G. Metz, X. Wu and S. O. Smith, J. Magn. Reson., Ser. A 110, 219 (1994).

(159) S. Zhang, C. L. Czekaj and W. T. Ford, J. Magn. Reson., Ser. A 111, 87 (1994).

(160) S. Hediger, B. H. Meier and R. R. Ernst, Chem. Phys. Lett. 240, 449 (1995).

(161) S. Hediger, P. Signer, M. Tomaselli, R. R. Ernst and B. H. Meier, J. Magn. Reson. 125, 291 (1997). 
(162) N. Zumbulyadis and J. M. O'Reilly, J. Magn. Reson. 82, 613 (1989).

(163) G. Jeschke and G. Grossmann, J. Magn. Reson., Ser. A 103, 323 (1993).

(164) G. Engelhardt and D. Michel, High-Resolution Solid-State NMR of Silicates and Zeolites (John Wiley and Sons, Norwich, England, 1987).

(165) C. M. Carter, D. W. Alderman and D. M. Grant, J. Magn. Reson. 65, 183 (1985).

(166) C. M. Carter, D. W. Alderman and D. M. Grant, J. Magn. Reson. 73, 114 (1987).

(167) M. H. Sherwood, D. W. Alderman and D. M. Grant, J. Magn. Reson. 84, 466 (1989).

(168) A. Bax, N. M. Szeverenyi and G. E. Maciel, J. Magn. Reson. 52, 147 (1983).

(169) A. C. Kolbert and R. G. Griffin, Chem. Phys. Lett. 166, 87 (1990).

(170) A. Bax, N. M. Szeverenyi and G. E. Maciel, J. Magn. Reson. 55, 494 (1983).

(171) T. Terao, T. Fujii, T. Onodera and A. Saika, Chem. Phys. Lett. 107, 145 (1984).

(172) G. E. Maciel, N. M. Szeverenjy and M. Saradashti, J. Magn. Reson. 64, 365 (1985).

(173) L. Frydman, G. C. Chingas, Y. K. Lee, P. J. Grandinetti, M. A. Eastman, G. A. Barrall and A. Pines, J. Chem. Phys. 97, 4800 (1992).

(174) A. C. Kolbert, H. J. M. de Groot and R. G. Griffin, J. Magn. Reson. 85, 60 (1989).

(175) R. C. Zeigler, R. A. Wind and G. E. Maciel, J. Magn. Reson. 79, 299 (1988).

(176) N. M. Szeverenyi, A. Bax and G. E. Maciel, J. Magn. Reson. 61, 440 (1985).

(177) L. Emsley and A. Pines In Proceedings of the International School of Physics (Enrico Fermi); Socièta Italiana di Fisica: Varenna on Lake Como, 1992.

(178) Z. Gan, J. Am. Chem. Soc. 114, 8307 (1992).

(179) S. L. Gann, J. H. Baltisberger and A. Pines, Chem. Phys. Lett. 210, 405 (1993).

(180) J. Z. Hu, D. W. Alderman, C. Ye, R. J. Pugmire and D. M. Grant, J. Magn. Reson., Ser. A 105, 82 (1993).

(181) S. L. Gann, Ph.D. Thesis, University of California, 1995.

(182) W. T. Dixon, J. Chem. Phys. 77, 1800 (1982).

(183) Z. Gan and R. R. Ernst, J. Magn. Reson., Ser. A 123, 140 (1996). 
(184) J. Z. Hu, A. M. Orendt, D. W. Alderman, C. Ye, R. J. Pugmire and D. M. Grant, Solid State NMR 2, 235 (1993).

(185) J. Z. Hu, W. Wang, F. Liu, M. S. Solum, D. W. Alderman, R. J. Pugmire and D. M. Grant, J. Magn. Reson., Ser. A 113, 210 (1995).

(186) J. Z. Hu, A. M. Orendt, D. W. Alderman, R. J. Pubmire, C. Ye and D. M. Grant, Solid State NMR 3, 181 (1994).

(187) W. T. Dixon, J. Schaefer, M. D. Sefcik, E. O. Stejskal and R. A. McKay, J. Magn. Reson. 49, 341 (1982).

(188) D. P. Raleigh, E. T. Olejniczak and R. G. Griffin, J. Chem. Phys. 89, 1333 (1988).

(189) S. J. Lang, J. Magn. Reson., Ser. A 104, 345 (1993).

(190) D. P. Raleigh, A. C. Kolbert and R. G. Griffin, J. Magn. Reson. 89, 1 (1990).

(191) H. Geen and G. Bodenhausen, J. Chem. Phys. 97, 2928 (1992).

(192) P. Zhang, P. J. Grandinetti and J. F. Stebbins, Journal of Physical Chemistry B 101, 4004 (1997).

(193) J. F. Stebbins In Mineral Physics and Crystallography::A Handbook of Physical Constants, AGU Reference Shelf 2; American Geophysical Union, 1995.

(194) J. F. Hinton, P. L. Guthrie, P. Pulay and K. Wolinski, J. Magn. Reson., Ser. A 103, 188 (1993).

(195) J. V. Smith, Feldspar Minerals (Springer-Verlag, Berlin, 1988).

(196) S. M. De Paul, M. Ernst, J. S. Shore, J. F. Stebbins and A. Pines, J. Phys. Chem. 101, 3240 (1997).

(197) T. K. Pratum and M. P. Klein, J. Magn. Reson. 55, 421 (1983).

(198) T. Gullion and J. Schaefer, J. Magn. Reson. 81, 196 (1989).

(199) T. Gullion and J. Schaefer, Adv. Magn. Reson. 13, 57 (1989).

(200) Y. Pan, T. Gullion and J. Schaefer, J. Magn. Reson. 90, 330 (1990).

(201) K. T. Mueller, T. P. Jarvie, D. J. Aurentz and B. W. Roberts, Chem. Phys. Lett. 242, 535 (1995).

(202) J. R. Garbow and T. Gullion, J. Magn. Reson. 95, 442 (1991).

(203) A. L. Blumenfeld, D. J. Coster and J. J. Friapat, Chem. Phys. Lett. 231, 491 (1994). 
(204) C. Hudalla, H. Eckert and R. Dupree, J. Phys. Chem. 100, 15986 (1996).

(205) T. Gullion, J. Magn. Reson., Ser. A 117, 326 (1995).

(206) T. Gullion, Chem. Phys. Lett. 246, 325 (1995).

(207) C. A. Fyfe, K. T. Mueller, H. Grondey and K. C. Wong-Moon, Chem. Phys. Lett. 199, 198 (1992).

(208) C. P. Grey and B. S. A. Kumar, J. Am. Chem. Soc. 117, 9071 (1995).

(209) B. Q. Sun, Ph.D. Thesis, University of California, Berkeley, 1991.

(210) A. Samoson and A. Pines, Rev. Sci. Instrum. 60, 3239 (1989).

(211) B. Q. Sun, J. H. Baltisberger, Y. Wu, A. Samoson and A. Pines, Solid State NMR 1, 267 (1992).

(212) J.-P. Amoureux, C. Fernandez and L. Frydman, Chem. Phys. Lett. 259, 347 (1996).

(213) A. Wokaun and R. R. Ernst, J. Chem. Phys. 67, 1752 (1977).

(214) S. Vega and Y. Naor, J. Chem. Phys. 75, 75 (1981).

(215) S. P. Brown, S. J. Heyes and S. Wimperis, J. Magn. Reson., Ser. A 119, 280 (1996).

(216) S. H. Wang, Z. Xu, J. H. Baltisberger, L. M. Bull, J. F. Stebbins and A. Pines, Solid State NMR 8, 1 (1997).

(217) C. Fernandez and J. P. Amoureux, Chem. Phys. Lett. 242, 449 (1995).

(218) A. A. Maudsley and R. R. Ernst, Chem. Phys. Lett. 50, 368 (1977).

(219) P. Caravatti, L. Braunschweiler and R. R. Ernst, Chem. Phys. Lett. 100, 305 (1983).

(220) J. E. Roberts, S. Vega and R. G. Griffin, J. Am. Chem. Soc. 106, 2506 (1984).

(221) A. J. Vega, J. Am. Chem. Soc. 110, 1049 (1988).

(222) G. G. Almond, R. K. Harris and K. R. Franklin, Solid State NMR 6, 31 (1996).

(223) T. P. Jarvie, R. M. Wenslow and K. T. Mueller, J. Am. Chem. Soc. 117, 570 (1995).

(224) C. Fernandez and J. P. Amoureux, Solid State NMR 5, 315 (1996).

(225) H. M. Ondik, Acta Cryst. 18, 226 (1965).

(226) H. Koller, G. Engelhardt, A. P. M. Kentgens and J. Sauer, J. Phys. Chem. 98, 1544 (1994). 
(227) A. Pines, M. G. Gibby and J. S. Waugh, J. Chem. Phys. 59, 569 (1973).

(228) G. Wu, D. Rovnyak and R. G. Griffin, J. Am. Chem. Soc. 118, 9326 (1996).

(229) E. L. Hahn, Phys. Rev. 80, 580 (1950).

(230) H. Schneider and H. Schmiedel, Phys. Lett. 30A, 298 (1969).

(231) W.-K. Rhim, A. Pines and J. S. Waugh, Phys. Rev. Lett. 25, 218 (1970).

(232) A. Llor, Z. Olejniczak, J. Sachleben and A. Pines, Phys. Rev. Lett. 67, 1989 (1991).

(233) N. Bloembergen, Physica (Utrecht) 15, 386 (1949).

(234) S. Zhang, B. H. Meier and R. R. Ernst, Phys. Rev. Lett. 69, 2149 (1992).

(235) T. Karlsson, M. Helmle, N. D. Kurur and M. H. Levitt, Chem. Phys. Lett. 247, 534 (1995).

(236) M. Tomaselli, S. Hediger, D. Suter and R. R. Ernst, J. Chem. Phys. 105, 10672 (1996).

(237) E. R. Andrew, A. Bradbury and R. G. Eades, Nature 182, 1659 (1958).

(238) I. J. Lowe, Phys. Rev. Lett. 2, 285 (1959).

(239) D. P. Raleigh, M. H. Levitt and R. G. Griffin, Chem. Phys. Lett. 146, 71 (1988).

(240) M. G. Colombo, B. H. Meier and R. R. Ernst, Chem. Phys. Lett. 146, 189 (1988).

(241) M. Goldman, Spin Temperature and Nuclear Magnetic Resonance in Solids (Oxford University Press, London, 1970).

(242) M. Ernst and B. H. Meier In Solid State NMR of Polymers; I. Ando and T. Askura, Eds., Elsevier Science Publisher, 1997, .

(243) H. Schmiedel, B. Hillner and S. Grande, Phys. Lett. 78A, 458 (1980).

(244) P. Robyr, B. H. Meier and R. R. Ernst, Chem. Phys. Lett. 162, 417 (1989).

(245) B. H. Meier, Adv. Magn. Opt. Reson. 18, 1 (1994).

(246) D. Suter and R. R. Ernst, Phys. Rev. B 32, 5608 (1985).

(247) A. Kubo and C. A. Mc Dowell, J. Chem. Phys. 89, 63 (1988).

(248) M. Linder, A. Hohener and R. R. Ernst, J. Chem. Phys. 73, 4959 (1980).

(249) P. M. Henrichs and M. Linder, J. Magn. Reson. 58, 458 (1984). 
(250) P. Robyr, M. Tomaselli, J. Straka, C. Grob-Pisano, U. W. Suter, B. H. Meier and R. R. Ernst, Mol. Phys. 84, 995 (1995).

(251) E. R. Andrew, A. Bradbury and R. G. Eades, Nature 183, 1802 (1959).

(252) M. Baldus, M. Tomaselli, B. H. Meier and R. R. Ernst, Chem. Phys. Lett. 130, 329 (1994).

(253) K. T. Mueller, B. Q. Sun, G. C. Chingas, J. W. Zwanziger, T. Terao and A. Pines, J. Magn. Reson. 86, 470 (1990).

(254) W. Nowacki, Helv. Chim. Acta 28, 1233 (1945).

(255) C. E. Bronniman, N. M. Szeverenyi and G. E. Maciel, J. Chem. Phys. 79, 3694 (1983). 